\title{
Representations by Certain Sextenary Quadratic Forms Whose Coefficients Are 1, 2, 3 and 6
}

\section{Barış Kendirli}

Istanbul Aydın University, Istanbul, Turkey

Email: bariskendirli@aydin.edu.tr

Received 9 December 2015; accepted 26 March 2016; published 29 March 2016

Copyright (C) 2016 by author and Scientific Research Publishing Inc.

This work is licensed under the Creative Commons Attribution International License (CC BY).

http://creativecommons.org/licenses/by/4.0/

(c) (i) Open Access

\section{Abstract}

Here, we determine formulae, for the numbers of representations of a positive integer by certain sextenary quadratic forms whose coefficients are 1, 2, 3 and 6.

\section{Keywords}

Sextenary Quadratic Forms, Representations, Theta Functions, Dedekind Eta Function, Eisenstein Series, Eisenstein Forms, Modular Forms, Cusp Forms

\section{Introduction}

The divisor function $\sigma_{i}(n)$ is defined for a positive integer $i$ by

$$
\begin{aligned}
& \sigma_{i}(n):=\sum_{d \text { positive integer }, d \mid n} d^{i} \text {, if } n \text { is a positive integer, and } \\
& \sigma_{i}(n):=0 \text { if } n \text { is not a positive integer. }
\end{aligned}
$$

The Dedekind eta function and the theta function are defined by

$$
\eta(z):=q^{1 / 24} \prod_{n=1}^{\infty}\left(1-q^{n}\right), \varphi(q):=\sum_{n \in \mathbb{Z}} q^{n^{2}},
$$

where

$$
q:=\mathrm{e}^{2 \pi i z}, z \in H=\{x+i y: y>0\},
$$

and an eta quotient of level $N$ is defined by 


$$
f(z):=\prod_{m \mid N} \eta(m z)^{a_{m}}, N, m \in \mathbb{N}, a_{m} \in Z .
$$

It is important and interesting to determine explicit formulas of the representation number of positive definite quadratic forms.

Here we give the following Lemma, see ([1], Theorem 1.64), about the modularity of an eta quotient.

Lemma 1. An eta quotient of level $N$ is a meromorphic modular form of weight $\frac{1}{2} \sum_{m \mid N} a_{m}$ on $\Gamma_{0}(N)$ having rational coefficients with respect to $q$ if

a) $\sum_{m \mid N} a_{m}$ is even,

b) $\prod_{m \mid N} m a_{m} \equiv \sum_{m \mid N} \frac{N}{m} a_{m} \equiv 0 \bmod 24$,

c) $\prod_{m \mid N} m^{a_{m}}$ is a square in $\mathbb{Q}$.

For $a_{1}, \cdots, a_{16} \in \mathbb{N}$ and a nonnegative integer $n$, we define

$$
N\left(a_{1}, \cdots, a_{16} ; n\right):=\operatorname{card}\left\{\left(x_{1}, \cdots, x_{16}\right) \in \mathbb{Z}^{16} \mid n=a_{1} x_{1}^{2}+\cdots+a_{16} x_{16}^{2}\right\} .
$$

Clearly $N\left(a_{1}, \cdots, a_{16} ; 0\right)=1$, and without loss of generality we can assume that $a_{1} \leq \cdots \leq a_{16}$.

Now, let's consider sextenary quadratic forms of the form

$$
Q:=x_{1}^{2}+\cdots+x_{a}^{2}+2\left(x_{a+1}^{2}+\cdots+x_{a+b}^{2}\right)+3\left(x_{a+b+1}^{2}+\cdots+x_{a+b+c}^{2}\right)+6\left(x_{a+b+c+1}^{2}+\cdots+x_{a+b+c+d=16}^{2}\right),
$$

where $a, b, c \in \mathbb{Z}, 0 \leq a \leq 16,0 \leq b \leq 16,0 \leq c \leq 16,0 \leq d \leq 16$.

We write $N\left(1^{a}, 2^{b}, 3^{c}, 6^{d} ; n\right)$ to denote the number of representations of $n$ by a sextenary quadratic form $(a, b, c, d)$. Its theta function is obviously

$$
\Theta_{Q}=\varphi^{a}(q) \varphi^{b}\left(q^{2}\right) \varphi^{c}\left(q^{3}\right) \varphi^{d}\left(q^{6}\right) .
$$

Formulae for $N\left(1^{2 i}, 2^{2 j}, 3^{2 k}, 6^{2 l} ; n\right)$ for the nine octonary quadratic forms $(2 i, 2 j, 2 k, 2 l)=(8,0,0,0),(2,6,0$, $0),(4,4,0,0),(6,2,0,0),(2,0,6,0),(4,0,4,0),(6,0,2,0),(4,0,0,4)$, and $(0,4,4,0)$ appear in the literature, (cf. [2]-[12]). Alaca and Williams have obtained some results on sextenary quadratic forms in terms of the functions $G_{4}$ and $H_{4}$, see [13] [14]. There are more works on representation number of sextenary quadratic forms in [15]-[17]. Other methods for representation number have been used in (cf. [7] [10] [12] [18] [19]). Here, we will classify all fourtuples $(a, b, c, d)$ for which $\Theta_{Q}$ is a modular form of weight 8 with level 24 . Then we will obtain their representation numbers in terms of the coefficients of Eisenstein series and some eta quotients.

First, by the following Theorem, we characterize the facts that

$$
\varphi^{a}(q) \varphi^{b}\left(q^{2}\right) \varphi^{c}\left(q^{3}\right) \varphi^{d}\left(q^{6}\right)
$$

are in $M_{8}\left(\Gamma_{0}(24)\right)$.

Theorem 1. Let

$$
Q:=x_{1}^{2}+\cdots+x_{a}^{2}+2\left(x_{a+1}^{2}+\cdots+x_{a+b}^{2}\right)+3\left(x_{a+b+1}^{2}+\cdots+x_{a+b+c}^{2}\right)+6\left(x_{a+b+c+1}^{2}+\cdots+x_{a+b+c+d=16}^{2}\right)
$$

where, $a, b, c \in \mathbb{Z}, 0 \leq a \leq 16,0 \leq b \leq 16,0 \leq c \leq 16,0 \leq d \leq 16$, be a sextenary quadratic form. Then its theta series is of the form

$$
\begin{aligned}
\Theta_{Q} & =\varphi^{a}(q) \varphi^{b}\left(q^{2}\right) \varphi^{c}\left(q^{3}\right) \varphi^{d}\left(q^{6}\right) \\
& =\eta^{-2 a}(q) \eta^{5 a-2 b}\left(q^{2}\right) \eta^{-2 c}\left(q^{3}\right) \eta^{5 b-2 a}\left(q^{4}\right) \eta^{5 c-2 d}\left(q^{6}\right) \eta^{-2 b}\left(q^{8}\right) \eta^{5 d-2 c}\left(q^{12}\right) \eta^{-2 d}\left(q^{24}\right) .
\end{aligned}
$$

Moreover, it is in $M_{8}\left(\Gamma_{0}(24)\right)$ if and only if $(a, b, c, d)$ is given in the Table 1. Here we also see that $a, b, c, d$ are either both even or both odd.

Proof. It follows from the Lemma 1, holomorphicity criterion in ([20] Corollary 2.3, p. 37) and the fact 
Table 1. Sextenary quadratic forms

\begin{tabular}{|c|c|c|c|}
\hline (0 222 12), & (0 24 10), & (0 26 ) & $\left(\begin{array}{llll}0 & 2 & 8 & 6\end{array}\right)$ \\
\hline (0 210 4), & (0 $\left.\begin{array}{llll}0 & 1 & 2\end{array}\right)$, & (0 214 0), & $\left(\begin{array}{llll}0 & 4 & 2 & 10\end{array}\right)$ \\
\hline (0 44 8), & (0 46 6), & (0 484 ), & $\left(\begin{array}{llll}0 & 4 & 10 & 2\end{array}\right)$ \\
\hline (0 412 0), & (0 62 8), & (0 64 6), & $\left(\begin{array}{llll}0 & 6 & 6 & 4\end{array}\right)$ \\
\hline (0 $\left.\begin{array}{llll}6 & 8 & 2\end{array}\right)$ & $\left(\begin{array}{llll}0 & 6 & 10 & 0\end{array}\right)$, & (0 82 6), & $(08444)$ \\
\hline (0 $\left.8 \begin{array}{lll}0 & 6 & 2\end{array}\right)$ & (0 $\left.8 \begin{array}{lll}8 & 0\end{array}\right)$ & ( $\left.\begin{array}{llll}0 & 10 & 2 & 4\end{array}\right)$ & $\left(\begin{array}{llll}0 & 10 & 4 & 2\end{array}\right)$ \\
\hline$\left(\begin{array}{llll}0 & 10 & 6 & 0\end{array}\right)$ & 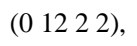 & ( $0124 \quad$ 0), & $\left(\begin{array}{llll}0 & 14 & 2 & 0\end{array}\right)$ \\
\hline (1 11113$)$, & (1 113 11), & (1 1159 ), & $(1177)$ \\
\hline (1 1195 ), & (1 11113 ), & (1 1113 1), & $\left(\begin{array}{llll}1 & 3 & 1 & 11\end{array}\right)$ \\
\hline (1 33 9), & (1 35 7), & (1 37 5), & (1 393 ) \\
\hline (1 311 1), & (1 519 ), & (1 553 7), & $(1555)$ \\
\hline (1 57 3), & (159 1), & (1 7 1 7), & $(1735)$ \\
\hline (1 75 3), & (1 77 1), & (1 $\left.9 \begin{array}{lll}9 & 1\end{array}\right)$ & (1 93 3) \\
\hline (1951), & (1 1111 3), & (1 1113 1), & $\left(\begin{array}{llll}1 & 13 & 1 & 1\end{array}\right)$ \\
\hline (2 $\left.\begin{array}{llll}2 & 0 & 14\end{array}\right)$, & (2 02 12), & (2 04 10), & $\left(\begin{array}{llll}2 & 0 & 6 & 8\end{array}\right)$ \\
\hline (2 $\left.0 \begin{array}{ll}0 & 6\end{array}\right)$ & (2 010 4), & ( $\left.\begin{array}{llll}2 & 0 & 12 & 2\end{array}\right)$ & $\left(\begin{array}{llll}2 & 0 & 14 & 0\end{array}\right)$ \\
\hline (2 220 12), & (2 22 10), & (2 24 8), & (2 266 ) \\
\hline (2 28 4), & (2 210 2), & (2 2212 0), & $\left(\begin{array}{llll}2 & 4 & 0 & 10\end{array}\right)$ \\
\hline (2 42 8), & (2 44 6), & (2 46 4), & $\left(\begin{array}{l}2 \\
4\end{array} 8\right.$ 2) \\
\hline (2 $\left.4 \begin{array}{lll}10 & 0\end{array}\right)$ & (2 $\left.\begin{array}{llll} & 0 & 8\end{array}\right)$ & (2 $\left.6 \begin{array}{ll}2 & 6\end{array}\right)$ & (2 644$)$ \\
\hline (2 $\left.6 \begin{array}{lll}6 & 6 & 2\end{array}\right)$ & (2 $\left.6 \begin{array}{ll}8 & 0\end{array}\right)$ & (2 80 6), & $\left(\begin{array}{llll}2 & 8 & 2 & 4\end{array}\right)$ \\
\hline (2 84 2), & (2 $\left.8 \begin{array}{lll}6 & 0\end{array}\right)$ & 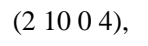 & $\left(\begin{array}{llll}2 & 10 & 2 & 2\end{array}\right)$ \\
\hline (2 104 0), & (2 $12 \quad 0$ 2), & (2 $12 \quad 2$ 0), & $\left(\begin{array}{llll}2 & 14 & 0 & 0\end{array}\right)$ \\
\hline (3 111 11), & (3 113 9), & (3 15 7), & (3 17 5) \\
\hline (3 19 3), & (3 1111 1), & (3 31 9), & (3 33 7) \\
\hline (3 35 5), & (3 37 3), & (3 39 1), & (3 517 ) \\
\hline (3 53 5), & (3 55 3), & (3 57 1), & (3 7 1 5) \\
\hline (3 7 3 3), & (3 75 1), & (3 $9 \begin{array}{lll}3 & \text { ), }\end{array}$ & (3 93 1) \\
\hline (3 11111 ), & (4 $\left.\begin{array}{llll}4 & 0 & 12\end{array}\right)$ & (4 02 ll), & $\left(\begin{array}{l}4 \\
0\end{array} 4\right.$ 8) \\
\hline (4 $\left.\begin{array}{llll}4 & 6 & 6\end{array}\right)$ & (4 $\left.\begin{array}{llll}0 & 8 & 4\end{array}\right)$ & $\left(\begin{array}{llll}4 & 0 & 1 & 10\end{array}\right)$ & $\left(\begin{array}{llll}4 & 0 & 12 & 0\end{array}\right)$ \\
\hline (4 20 10), & (4 222 8), & (4 24 6), & (4 264 ) \\
\hline (4 $\left.\begin{array}{lll}2 & 8 & 2\end{array}\right)$ & (4 210 0), & (4 40 8), & $(4426)$ \\
\hline (4 444 ), & (4 46 2), & (4 48 0), & $\left(\begin{array}{llll}4 & 6 & 0 & 6\end{array}\right)$ \\
\hline (4 $\left.6 \begin{array}{lll}6 & 4\end{array}\right)$ & (4 64 2), & (4 $\left.6 \begin{array}{lll}6 & 0 & 0\end{array}\right)$ & $\left(\begin{array}{llll}4 & 8 & 0 & 4\end{array}\right)$ \\
\hline (4 $\left.\begin{array}{llll}8 & 2 & 2\end{array}\right)$, & (4 84 0), & 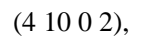 & $\left(\begin{array}{llll}4 & 10 & 2 & 0\end{array}\right)$ \\
\hline$\left(\begin{array}{llll}4 & 12 & 0 & 0\end{array}\right)$ & (5 11119 ), & (5 $\left.1 \begin{array}{lll}3 & 3 & 7\end{array}\right)$ & (5 155$)$ \\
\hline (5 17 3), & (5 19 1), & (5 $3 \quad 1$ 7), & (5 33 5) \\
\hline (5 35 3), & (5 37 1), & (5 5515$)$ & (5 53 3) \\
\hline (5 55 1), & (5 71 3), & (5 73 1), & 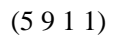 \\
\hline
\end{tabular}




\begin{tabular}{|c|c|c|c|}
\hline Continued & & & \\
\hline$\left(\begin{array}{llll}6 & 0 & 0 & 10\end{array}\right)$, & $\left(\begin{array}{llll}6 & 0 & 2 & 8\end{array}\right)$ & ( $\left.\begin{array}{lllll}6 & 0 & 4 & 6\end{array}\right)$ & $\left(\begin{array}{llll}6 & 0 & 6 & 4\end{array}\right)$ \\
\hline (6 $\left.\begin{array}{llll}6 & 0 & 2\end{array}\right)$ & $\left(\begin{array}{llll}6 & 0 & 10 & 0\end{array}\right)$ & (6 $\left.\begin{array}{llll}6 & 0 & 8\end{array}\right)$ & 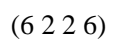 \\
\hline (6 254 4), & (6 $\left.\begin{array}{lll}6 & 6 & 2\end{array}\right)$ & (6 $\left.\begin{array}{llll}6 & 8 & 0\end{array}\right)$ & $\left(\begin{array}{llll}6 & 4 & 0 & 6\end{array}\right)$ \\
\hline (6 $\left.4 \begin{array}{lll}6 & 4\end{array}\right)$ & (6 44 2), & (6 46 o), & $\left(\begin{array}{llll}6 & 6 & 0 & 4\end{array}\right)$ \\
\hline (6 $\left.6 \begin{array}{lll}6 & 2 & 2\end{array}\right)$ & (6 64 0), & ( (6 $\left.\begin{array}{lll}8 & 0 & 2\end{array}\right)$, & $\left(\begin{array}{llll}6 & 8 & 2 & 0\end{array}\right)$ \\
\hline$\left(\begin{array}{llll}6 & 10 & 0 & 0\end{array}\right)$, & (7 1117 ), & (7 1135$)$ & $(7153)$ \\
\hline (7 17 1), & (7 3 1 5), & (7 3 3 3), & $(7351)$ \\
\hline (7 $\left.5 \begin{array}{lll}5 & 1 & 3\end{array}\right)$ & (7 53 1), & (7 $\left.7 \begin{array}{lll}1 & 1\end{array}\right)$ & $\left(\begin{array}{llll}8 & 0 & 0 & 8\end{array}\right)$ \\
\hline ( (8 $\left.\begin{array}{lll}8 & 2 & 6\end{array}\right)$, & (8 004 4), & $\left(\begin{array}{llll}8 & 0 & 6 & 2\end{array}\right)$ & $\left(\begin{array}{llll}8 & 0 & 8 & 0\end{array}\right)$ \\
\hline (8 20 c 0 6), & (8 252 4), & (8 $\left.\begin{array}{llll} & 4 & 4\end{array}\right)$ & $\left(\begin{array}{llll}8 & 2 & 6 & 0\end{array}\right)$ \\
\hline (8 40 4), & (8 $\left.4 \begin{array}{lll}2 & 2\end{array}\right)$ & (8 44 0), & $\left(\begin{array}{llll}8 & 6 & 0 & 2\end{array}\right)$ \\
\hline (8 $\left.6 \begin{array}{lll}8 & 2 & 0\end{array}\right)$, & ( (8 $\left.8 \begin{array}{lll}8 & 0 & 0\end{array}\right)$ & (9 1115$)$ & (9 $\left.\begin{array}{llll} & 1 & 1 & 3\end{array}\right)$ \\
\hline (9 15 1), & (9 $\left.\begin{array}{llll}9 & 1 & 3\end{array}\right)$ & (9 33 1), & (9 511 1) \\
\hline 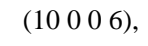 & $\left(\begin{array}{llll}1 & 0 & 0 & 2\end{array}\right)$ & $\left(\begin{array}{llll}1 & 0 & 4 & 4\end{array}\right)$ & $\left(\begin{array}{llll}10 & 0 & 6 & 0\end{array}\right)$ \\
\hline (10 20 4), & (10 22 2), & (10 24 0), & $\left(\begin{array}{lll}10 & 4 & 0\end{array}\right)$ \\
\hline (10 42 0), & 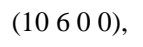 & (11 11113$)$ & $\left(\begin{array}{llll}1 & 1 & 3 & 1\end{array}\right)$ \\
\hline (11 3 1 1), & (12 0004$)$ & (12 02 2), & $\left(\begin{array}{llll}1 & 0 & 4 & 0\end{array}\right)$ \\
\hline (12 20 2), & (12 22 0), & (12 40 0), & (13 1111 ) \\
\hline$\left(\begin{array}{llll}14 & 0 & 0 & 2\end{array}\right)$, & (14 02 0), & (14 200 0), & 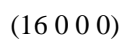 \\
\hline
\end{tabular}

that

$$
\varphi(q)=\frac{\eta^{5}\left(q^{2}\right)}{\eta^{2}(q) \eta^{2}\left(q^{4}\right)} .
$$

The condition $1^{a_{1}} 2^{a_{2}} 3^{a_{3}} 4^{a_{4}} 6^{a_{6}} 8^{a_{8}} 12^{a_{12}} 24^{a_{24}}$ is a square of a rational number implies that either $a, b, c, d$ are both even or both odd integers.

Now let,

$$
\begin{gathered}
E_{8}(q)=1+480 \sum_{n=1}^{\infty} \sigma_{7}(n) q^{n}, \\
A_{1}(q):=\eta(z)^{8} \eta(2 z)^{8}=q \prod_{n=1}^{\infty}\left(1-q^{n}\right)^{8}\left(1-q^{2 n}\right)^{8}, \\
A_{2}(q):=\frac{\eta(2 z)^{30} \eta(4 z)^{2}}{\eta(z)^{12} \eta(8 z)^{4}}=q \prod_{n=1}^{\infty} \frac{\left(1-q^{2 n}\right)^{30}\left(1-q^{4 n}\right)^{2}}{\left(1-q^{n}\right)^{12}\left(1-q^{8 n}\right)^{4}}, \\
A_{3}(q):=\frac{\eta(2 z)^{16} \eta(4 z)^{16}}{\eta(z)^{8} \eta(8 z)^{8}}=q \prod_{n=1}^{\infty} \frac{\left(1-q^{2 n}\right)^{16}\left(1-q^{4 n}\right)^{16}}{\left(1-q^{n}\right)^{8}\left(1-q^{8 n}\right)^{8}},
\end{gathered}
$$

$\Delta_{3,8}$ the unique newform in $S_{8}\left(\Gamma_{0}(3)\right)$,

$$
A_{4}(q):=\frac{\eta(2 z)^{26} \eta(3 z)^{6}}{\eta(z)^{10} \eta(6 z)^{6}}=q \prod_{n=1}^{\infty} \frac{\left(1-q^{2 n}\right)^{26}\left(1-q^{3 n}\right)^{6}}{\left(1-q^{n}\right)^{10}\left(1-q^{6 n}\right)^{6}},
$$




$$
\begin{aligned}
A_{5} & :=\frac{\eta(2 z)^{8} \eta(3 z)^{4} \eta(12 z)^{8}}{\eta(z)^{4}}=q^{5} \prod_{n=1}^{\infty} \frac{\left(1-q^{2 n}\right)^{8}\left(1-q^{3 n}\right)^{4}\left(1-q^{12 n}\right)^{8}}{\left(1-q^{n}\right)^{4}}, \\
A_{6}:=\frac{\eta(z)^{14} \eta(12 z)^{8}}{\eta(2 z)^{4} \eta(3 z)^{2}}=q^{4} \prod_{n=1}^{\infty} \frac{\left(1-q^{n}\right)^{14}\left(1-q^{12 n}\right)^{8}}{\left(1-q^{2 n}\right)^{4}\left(1-q^{3 n}\right)^{2}}, & :=\frac{\eta(2 z)^{3} \eta(3 z)^{4} \eta(4 z)^{4} \eta(6 z)^{3} \eta(8 z)^{3} \eta(12 z)^{4}}{\eta(z)^{4} \eta(24 z)} \\
& =q^{4} \prod_{n=1}^{\infty} \frac{\left(1-q^{2 n}\right)^{3}\left(1-q^{3 n}\right)^{4}\left(1-q^{4 n}\right)^{4}\left(1-q^{6 n}\right)^{3}\left(1-q^{8 n}\right)^{3}\left(1-q^{12 n}\right)^{4}}{\left(1-q^{n}\right)^{4}\left(1-q^{24 n}\right)}, \\
A_{8} & :=q^{6} \frac{\eta(2 z)^{3} \eta(3 z)^{4} \eta(4 z)^{5} \eta(6 z)^{3} \eta(8 z)^{4} \eta(24 z)^{4}}{\eta(z)^{4} \eta(12 z)^{3}} \\
& =q^{6} \prod_{n=1}^{\infty} \frac{\left(1-q^{2 n}\right)^{3}\left(1-q^{3 n}\right)^{4}\left(1-q^{4 n}\right)^{5}\left(1-q^{6 n}\right)^{3}\left(1-q^{8 n}\right)^{4}\left(1-q^{24 n}\right)^{4}}{\left(1-q^{n}\right)^{4}\left(1-q^{12 n}\right)^{3}}, \\
A_{9}:= & q^{5} \frac{\eta(2 z)^{4} \eta(3 z)^{4} \eta(4 z) \eta(6 z)^{4} \eta(8 z)^{5} \eta(12 z) \eta(24 z)}{\eta(z)^{4}} \\
= & q^{5} \prod_{n=1}^{\infty} \frac{\left(1-q^{2 n}\right)^{4}\left(1-q^{3 n}\right)^{4}\left(1-q^{4 n}\right)\left(1-q^{6 n}\right)^{4}\left(1-q^{8 n}\right)^{5}\left(1-q^{12 n}\right)\left(1-q^{24 n}\right)}{\left(1-q^{n}\right)^{4}} .
\end{aligned}
$$

Theorem 2. The set

$$
\begin{aligned}
& \left\{E_{8}, E_{8}(2 z), E_{8}(3 z), E_{8}(4 z), E_{8}(6 z), E_{8}(8 z), E_{8}(12 z), E_{8}(24 z),\right. \\
& A_{1}, A_{1}(2 z), A_{1}(3 z), A_{1}(4 z), A_{1}(6 z), A_{1}(12 z), A_{2}, A_{2}(3 z), A_{3}, \\
& A_{3}(3 z), \Delta_{3,8}, \Delta_{3,8}(2 z), \Delta_{3,8}(4 z), \Delta_{3,8}(8 z), A_{4}, A_{4}(2 z), A_{4}(4 z), \\
& \left.A_{5}, A_{5}(2 z), A_{6}, A_{6}(2 z), A_{7}, A_{8}, A_{9}\right\}
\end{aligned}
$$

is a basis of $M_{8}\left(\Gamma_{0}(24)\right)$. Moreover, the unique newform in $S_{8}\left(\Gamma_{0}(2)\right)$ is $A_{1}$, the unique newform in $S_{8}\left(\Gamma_{0}(6)\right)$ is $A_{4}$, the two unique newforms in $S_{8}\left(\Gamma_{0}(8)\right)$ are

$$
\begin{gathered}
\Delta_{8,8,1}=-A_{1}-24 A_{1}(2 z)-256 A_{1}(4 z)+2 A_{3}, \\
\Delta_{8,8,2}=-A_{1}-8 A_{1}(2 z)+256 A_{1}(4 z)-4 A_{2}+6 A_{3},
\end{gathered}
$$

the two unique newforms in $S_{8}\left(\Gamma_{0}(12)\right)$ are

$$
\begin{aligned}
\Delta_{12,8,1} & =-\frac{23}{9} A_{1}-\frac{304}{45} A_{1}(2 z)-81 A_{1}(3 z)-\frac{1296}{5} A_{1}(6 z)-\frac{744}{5} \Delta_{3,8}(2 z) \\
-\frac{864}{5} & \Delta_{3,8}(4 z)+\frac{32}{9} A_{4}+\frac{896}{9} A_{4}(2 z)-864 A_{5}-48 A_{6}, \\
\Delta_{12,8,2}(q)= & -\frac{49}{45} A_{1}-\frac{64}{45} A_{1}(2 z)-\frac{81}{5} A_{1}(3 z)+\frac{5184}{5} A_{1}(6 z)+\frac{6}{5} \Delta_{3,8}-\frac{828}{5} \Delta_{3,8}(2 z) \\
& -\frac{1344}{5} \Delta_{3,8}(4 z)+\frac{8}{9} A_{4}+\frac{1280}{9} A_{4}(2 z)-1728 A_{5}-48 A_{6},
\end{aligned}
$$


and the three unique newforms in $S_{8}\left(\Gamma_{0}(24)\right)$ are

$$
\begin{aligned}
\Delta_{24,8,1}= & -\frac{799}{171} A_{1}-\frac{2984}{285} A_{1}(2 z)+\frac{2511}{19} A_{1}(3 z)+\frac{282368}{855} A_{1}(4 z)+\frac{206712}{95} A_{1}(6 z) \\
& +\frac{2426112}{95} A_{1}(12 z)-\frac{12}{19} A_{2}+\frac{968}{95} A_{2}(3 z)+\frac{313536}{95} A_{3}-\frac{69632}{95} A_{3}(3 z) \\
& +\frac{736}{171} \Delta_{3,8}-\frac{4352}{57} \Delta_{3,8}(2 z)-\frac{16384}{9} \Delta_{3,8}(4 z)-4 \Delta_{3,8}(8 z)-\frac{1620}{19} A_{4}+6 A_{4}(2 z) \\
& -\frac{1458}{19} A_{4}(4 z)+\frac{41472}{19} A_{5}+\frac{304128}{19} A_{5}(2 z)+\frac{15360}{19} A_{6}(2 z)-\frac{18432}{19} A_{7} \\
& +\frac{15360}{19} A_{8}+\frac{27648}{19} A_{9}, \\
\Delta_{24,8,2}= & \frac{33187}{7695} A_{1}-\frac{29000}{171} A_{1}(2 z)+\frac{6363}{95} A_{1}(3 z)+\frac{6506752}{7695} A_{1}(4 z)-\frac{22968}{19} A_{1}(6 z) \\
- & \frac{2573568}{95} A_{1}(12 z)+\frac{148}{855} A_{2}+\frac{21608}{855} A_{2}(3 z)-\frac{7839808}{855} A_{3}-\frac{16928768}{855} A_{3}(3 z) \\
+ & \frac{4832}{1539} \Delta_{3,8}+\frac{10496}{171} \Delta_{3,8}(2 z)+\frac{704512}{81} \Delta_{3,8}(4 z)+\frac{4320}{19} A_{4}+2 A_{4}(2 z) \\
- & \frac{7398}{19} A_{4}(4 z)+\frac{20736}{19} A_{5}-\frac{1569792}{19} A_{5}(2 z)-\frac{60416}{19} A_{6}(2 z)-\frac{48128}{19} A_{7} \\
- & \frac{60416}{19} A_{8}+\frac{140288}{19} A_{9}, \\
& +3200 A_{9} . \\
& +\frac{113}{162} A_{1}-\frac{12764}{135} A_{1}(2 z)-\frac{279}{2} A_{1}(3 z)+\frac{228352}{405} A_{1}(4 z)-\frac{13212}{5} A_{1}(6 z) \\
& +\frac{18432}{5} A_{1}(12 z)+\frac{13}{18} A_{2}+\frac{413}{45} A_{2}(3 z)-\frac{82880}{9} A_{3}-\frac{674048}{45} A_{3}(3 z) \\
& -\frac{196}{81} \Delta_{3,8}+\frac{2560}{27} \Delta_{3,8}(2 z)+\frac{603136}{81} \Delta_{3,8}(4 z)-216 A_{4}+2 A_{4}(2 z) \\
& +378 A_{4}(4 z)-1584 A_{5}-79104 A_{5}(2 z)-2816 A_{6}(2 z)-512 A_{7}-2816 A_{8} \\
&
\end{aligned}
$$

Proof. $M_{8}\left(\Gamma_{0}(24)\right)$ is 32 dimensional, $S_{8}\left(\Gamma_{0}(24)\right)$ is 24 dimensional, see ([21] Chapter 3, p. 87 and Chapter 5, p. 197), and generated by

$$
\begin{gathered}
\Delta_{2,8}(z), \Delta_{2,8}(2 z), \Delta_{2,8}(3 z), \Delta_{2,8}(4 z), \Delta_{2,8}(6 z), \Delta_{2,8}(12 z) \\
\Delta_{3,8}(z), \Delta_{3,8}(2 z), \Delta_{3,8}(4 z), \Delta_{3,8}(8 z), \\
\Delta_{6,8}(z)=(-10,26,6,-6), \Delta_{6,8}(2 z), \Delta_{6,8}(4 z), \\
\Delta_{8,8,1}(z), \Delta_{8,8,1}(3 z), \Delta_{8,8,2}(z), \Delta_{8,8,2}(3 z), \\
\Delta_{12,8,1}(z), \Delta_{12,8,1}(3 z), \Delta_{12,8,2}(z), \Delta_{12,8,2}(3 z), \\
\Delta_{24,8,1}(z), \Delta_{24,8,2}(z), \Delta_{24,8,3}(z)
\end{gathered}
$$

where $\Delta_{2,8}$ is the unique newform in $S_{8}\left(\Gamma_{0}(2)\right) ; \Delta_{3,8}$ is the unique newform in $S_{8}\left(\Gamma_{0}(3)\right) ; \Delta_{6,8}$ is the unique newform in $S_{8}\left(\Gamma_{0}(6)\right), \Delta_{8,8,1}, \Delta_{8,8,2}$ are the unique newforms in $S_{8}\left(\Gamma_{0}(8)\right) ; \Delta_{12,8,1}, \Delta_{12,8,2}$ are the unique newforms in $S_{8}\left(\Gamma_{0}(12)\right)$ and $\Delta_{24,8,1}, \Delta_{24,8,2}, \Delta_{24,8,3}$ are the unique newforms in $S_{8}\left(\Gamma_{0}(24)\right)$.

As a consequence of this Theorem, we have obtained the following Corollary.We have used Magma for the 
calculations.

\section{Corollary}

The following representation numbers formulae are valid.

$$
\begin{aligned}
& N\left(2^{2}, 3^{2}, 6^{8} ; n\right)=\frac{1}{53529600} \sigma_{7}(n)-\frac{1}{53529600} \sigma_{7}\left(\frac{n}{2}\right)-\frac{547}{17843200} \sigma_{7}\left(\frac{n}{3}\right)+\frac{1}{418200} \sigma_{7}\left(\frac{n}{4}\right) \\
& +\frac{547}{17843200} \sigma_{7}\left(\frac{n}{6}\right)-\frac{32}{52275} \sigma_{7}\left(\frac{n}{8}\right)-\frac{547}{139400} \sigma_{7}\left(\frac{n}{12}\right)+\frac{17504}{17425} \sigma_{7}\left(\frac{n}{24}\right) \\
& -\frac{24851479}{62791200} a_{1}(n)+\frac{75407}{15390} a_{1}\left(\frac{n}{2}\right)+\frac{3035817}{258400} a_{1}\left(\frac{n}{3}\right)-\frac{22005632}{654075} a_{1}\left(\frac{n}{4}\right) \\
& +\frac{26007}{190} a_{1}\left(\frac{n}{6}\right)+\frac{26971008}{8075} a_{1}\left(\frac{n}{12}\right)-\frac{1218751}{8413200} \Delta_{3,8}(n)-\frac{1913293}{4206600} \Delta_{3,8}\left(\frac{n}{2}\right) \\
& +\frac{118785814}{175275} \Delta_{3,8}\left(\frac{n}{4}\right)+\frac{117613184}{175275} \Delta_{3,8}\left(\frac{n}{8}\right)+\frac{24961}{46170} a_{4}(n)-\frac{9464}{1539} a_{4}\left(\frac{n}{2}\right) \\
& -\frac{214528}{405} a_{4}\left(\frac{n}{4}\right)-\frac{1}{2} a_{2}(n)-\frac{411}{38} a_{2}\left(\frac{n}{3}\right)+\frac{1}{2} a_{3}(n)-\frac{45}{38} a_{3}\left(\frac{n}{3}\right)+\frac{14707}{57} a_{5}(n) \\
& +\frac{303488}{57} a_{5}\left(\frac{n}{2}\right)-\frac{1}{2} a_{6}(n)+\frac{3840}{19} a_{6}\left(\frac{n}{2}\right)+\frac{1072}{57} a_{7}(n)+\frac{6272}{19} a_{8}(n)-\frac{5248}{19} a_{9}(n), \\
& N\left(2^{2}, 3^{4}, 6^{10} ; n\right)=\frac{1}{26764800} \sigma_{7}(n)-\frac{1}{26764800} \sigma_{7}\left(\frac{n}{2}\right)-\frac{547}{8921600} \sigma_{7}\left(\frac{n}{3}\right)+\frac{1}{418200} \sigma_{7}\left(\frac{n}{4}\right) \\
& +\frac{547}{8921600} \sigma_{7}\left(\frac{n}{6}\right)-\frac{32}{52275} \sigma_{7}\left(\frac{n}{8}\right)-\frac{547}{139400} \sigma_{7}\left(\frac{n}{12}\right)+\frac{17504}{17425} \sigma_{7}\left(\frac{n}{24}\right)-\frac{24851479}{31395600} a_{1}(n) \\
& +\frac{271669}{23085} a_{1}\left(\frac{n}{2}\right)+\frac{3035817}{129200} a_{1}\left(\frac{n}{3}\right)-\frac{5172928}{72675} a_{1}\left(\frac{n}{4}\right)+\frac{37863}{95} a_{1}\left(\frac{n}{6}\right)+\frac{50618688}{8075} a_{1}\left(\frac{n}{12}\right) \\
& -\frac{1218751}{4206600} \Delta_{3,8}(n)-\frac{4219133}{2103300} \Delta_{3,8}\left(\frac{n}{2}\right)+\frac{827129692}{525825} \Delta_{3,8}\left(\frac{n}{4}\right)+\frac{84171008}{58425} \Delta_{3,8}\left(\frac{n}{8}\right)+\frac{24961}{23085} a_{4}(n) \\
& -\frac{79280}{4617} a_{4}\left(\frac{n}{2}\right)-\frac{54272}{45} a_{4}\left(\frac{n}{4}\right)-a_{2}(n)-\frac{411}{19} a_{2}\left(\frac{n}{3}\right)+a_{3}(n)-\frac{45}{19} a_{3}\left(\frac{n}{3}\right)+\frac{29414}{57} a_{5}(n) \\
& +\frac{232320}{19} a_{5}\left(\frac{n}{2}\right)-a_{6}(n)+\frac{8896}{19} a_{6}\left(\frac{n}{2}\right)+\frac{2144}{57} a_{7}(n)+\frac{12544}{19} a_{8}(n)-\frac{10496}{19} a_{9}(n), \\
& N\left(2^{2}, 3^{6}, 6^{8} ; n\right)=\frac{1}{13382400} \sigma_{7}(n)-\frac{1}{13382400} \sigma_{7}\left(\frac{n}{2}\right)-\frac{547}{4460800} \sigma_{7}\left(\frac{n}{3}\right)+\frac{1}{418200} \sigma_{7}\left(\frac{n}{4}\right) \\
& +\frac{547}{4460800} \sigma_{7}\left(\frac{n}{6}\right)-\frac{32}{52275} \sigma_{7}\left(\frac{n}{8}\right)-\frac{547}{139400} \sigma_{7}\left(\frac{n}{12}\right)+\frac{17504}{17425} \sigma_{7}\left(\frac{n}{24}\right)-\frac{21863219}{15697800} a_{1}(n) \\
& +\frac{585584}{23085} a_{1}\left(\frac{n}{2}\right)+\frac{2649237}{64600} a_{1}\left(\frac{n}{3}\right)-\frac{93405632}{654075} a_{1}\left(\frac{n}{4}\right)+\frac{78408}{95} a_{1}\left(\frac{n}{6}\right)+\frac{96086208}{8075} a_{1}\left(\frac{n}{12}\right) \\
& -\frac{1063361}{2103300} \Delta_{3,8}(n)-\frac{2003201}{350550} \Delta_{3,8}\left(\frac{n}{2}\right)+\frac{1673520572}{525825} \Delta_{3,8}\left(\frac{n}{4}\right)+\frac{507670784}{175275} \Delta_{3,8}\left(\frac{n}{8}\right)+\frac{43822}{23085} a_{4}(n) \\
& -\frac{160432}{4617} a_{4}\left(\frac{n}{2}\right)-\frac{990208}{405} a_{4}\left(\frac{n}{4}\right)-2 a_{2}(n)-\frac{984}{19} a_{2}\left(\frac{n}{3}\right)+2 a_{3}(n)+\frac{300}{19} a_{3}\left(\frac{n}{3}\right)+\frac{52604}{57} a_{5}(n) \\
& +\frac{1419008}{57} a_{5}\left(\frac{n}{2}\right)-2 a_{6}(n)+\frac{18112}{19} a_{6}\left(\frac{n}{2}\right)+\frac{6176}{57} a_{7}(n)+\frac{24192}{19} a_{8}(n)-\frac{21632}{19} a_{9}(n) \text {, }
\end{aligned}
$$




$$
\begin{aligned}
& N\left(2^{2}, 3^{8}, 6^{6} ; n\right)=\frac{1}{6691200} \sigma_{7}(n)-\frac{1}{6691200} \sigma_{7}\left(\frac{n}{2}\right)-\frac{547}{2230400} \sigma_{7}\left(\frac{n}{3}\right) \\
& +\frac{1}{418200} \sigma_{7}\left(\frac{n}{4}\right)+\frac{547}{2230400} \sigma_{7}\left(\frac{n}{6}\right)-\frac{32}{52275} \sigma_{7}\left(\frac{n}{8}\right)-\frac{547}{139400} \sigma_{7}\left(\frac{n}{12}\right) \\
& +\frac{17504}{17425} \sigma_{7}\left(\frac{n}{24}\right)-\frac{6291653}{2616300} a_{1}(n)+\frac{243304}{4617} a_{1}\left(\frac{n}{2}\right)+\frac{2262657}{32300} a_{1}\left(\frac{n}{3}\right) \\
& -\frac{183404992}{654075} a_{1}\left(\frac{n}{4}\right)+\frac{30384}{19} a_{1}\left(\frac{n}{6}\right)+\frac{187674048}{8075} a_{1}\left(\frac{n}{12}\right)-\frac{302657}{350550} \Delta_{3,8}(n) \\
& -\frac{7021073}{525825} \Delta_{3,8}\left(\frac{n}{2}\right)+\frac{3270058112}{525825} \Delta_{3,8}\left(\frac{n}{4}\right)+\frac{996863104}{175275} \Delta_{3,8}\left(\frac{n}{8}\right)+\frac{25148}{7695} a_{4}(n) \\
& -\frac{305152}{4617} a_{4}\left(\frac{n}{2}\right)-\frac{1945088}{405} a_{4}\left(\frac{n}{4}\right)-4 a_{2}(n)-\frac{2292}{19} a_{2}\left(\frac{n}{3}\right)+4 a_{3}(n)+\frac{1380}{19} a_{3}\left(\frac{n}{3}\right) \\
& +\frac{30920}{19} a_{5}(n)+\frac{2788480}{57} a_{5}\left(\frac{n}{2}\right)-4 a_{6}(n)+\frac{35648}{19} a_{6}\left(\frac{n}{2}\right)+\frac{5376}{19} a_{7}(n) \\
& +\frac{46592}{19} a_{8}(n)-\frac{44544}{19} a_{9}(n) \text {, } \\
& N\left(2^{2}, 3^{10}, 6^{4} ; n\right)=\frac{1}{3345600} \sigma_{7}(n)-\frac{1}{3345600} \sigma_{7}\left(\frac{n}{2}\right)-\frac{547}{1115200} \sigma_{7}\left(\frac{n}{3}\right) \\
& +\frac{1}{418200} \sigma_{7}\left(\frac{n}{4}\right)+\frac{547}{1115200} \sigma_{7}\left(\frac{n}{6}\right)-\frac{32}{52275} \sigma_{7}\left(\frac{n}{8}\right)-\frac{547}{139400} \sigma_{7}\left(\frac{n}{12}\right) \\
& +\frac{17504}{17425} \sigma_{7}\left(\frac{n}{24}\right)-\frac{8283562}{1962225} a_{1}(n)+\frac{832268}{7695} a_{1}\left(\frac{n}{2}\right)+\frac{988401}{8075} a_{1}\left(\frac{n}{3}\right) \\
& -\frac{362533312}{654075} a_{1}\left(\frac{n}{4}\right)+\frac{292668}{95} a_{1}\left(\frac{n}{6}\right)+\frac{372644928}{8075} a_{1}\left(\frac{n}{12}\right)+\frac{1419008}{57} a_{5}\left(\frac{n}{2}\right) \\
& -2 a_{6}(n)+\frac{18112}{19} a_{6}\left(\frac{n}{2}\right)+\frac{6176}{57} a_{7}(n)+\frac{24192}{19} a_{8}(n)-\frac{21632}{19} a_{9}(n), \\
& N\left(2^{2}, 3^{8}, 6^{6} ; n\right)=\frac{1}{6691200} \sigma_{7}(n)-\frac{1}{6691200} \sigma_{7}\left(\frac{n}{2}\right)-\frac{547}{2230400} \sigma_{7}\left(\frac{n}{3}\right) \\
& +\frac{1}{418200} \sigma_{7}\left(\frac{n}{4}\right)+\frac{547}{2230400} \sigma_{7}\left(\frac{n}{6}\right)-\frac{32}{52275} \sigma_{7}\left(\frac{n}{8}\right)-\frac{547}{139400} \sigma_{7}\left(\frac{n}{12}\right) \\
& +\frac{17504}{17425} \sigma_{7}\left(\frac{n}{24}\right)-\frac{6291653}{2616300} a_{1}(n)+\frac{243304}{4617} a_{1}\left(\frac{n}{2}\right)+\frac{2262657}{32300} a_{1}\left(\frac{n}{3}\right) \\
& -\frac{183404992}{654075} a_{1}\left(\frac{n}{4}\right)+\frac{30384}{19} a_{1}\left(\frac{n}{6}\right)+\frac{187674048}{8075} a_{1}\left(\frac{n}{12}\right)-\frac{302657}{350550} \Delta_{3,8}(n) \\
& -\frac{7021073}{525825} \Delta_{3,8}\left(\frac{n}{2}\right)+\frac{3270058112}{525825} \Delta_{3,8}\left(\frac{n}{4}\right)+\frac{996863104}{175275} \Delta_{3,8}\left(\frac{n}{8}\right)+\frac{25148}{7695} a_{4}(n) \\
& -\frac{305152}{4617} a_{4}\left(\frac{n}{2}\right)-\frac{1945088}{405} a_{4}\left(\frac{n}{4}\right)-4 a_{2}(n)-\frac{2292}{19} a_{2}\left(\frac{n}{3}\right)+4 a_{3}(n)+\frac{1380}{19} a_{3}\left(\frac{n}{3}\right) \\
& +\frac{30920}{19} a_{5}(n)+\frac{2788480}{57} a_{5}\left(\frac{n}{2}\right)-4 a_{6}(n)+\frac{35648}{19} a_{6}\left(\frac{n}{2}\right)+\frac{5376}{19} a_{7}(n) \\
& +\frac{46592}{19} a_{8}(n)-\frac{44544}{19} a_{9}(n) \text {, }
\end{aligned}
$$




$$
\begin{aligned}
& N\left(2^{2}, 3^{10}, 6^{4} ; n\right)=\frac{1}{3345600} \sigma_{7}(n)-\frac{1}{3345600} \sigma_{7}\left(\frac{n}{2}\right)-\frac{547}{1115200} \sigma_{7}\left(\frac{n}{3}\right)+\frac{1}{418200} \sigma_{7}\left(\frac{n}{4}\right) \\
& +\frac{547}{1115200} \sigma_{7}\left(\frac{n}{6}\right)-\frac{32}{52275} \sigma_{7}\left(\frac{n}{8}\right)-\frac{547}{139400} \sigma_{7}\left(\frac{n}{12}\right)+\frac{17504}{17425} \sigma_{7}\left(\frac{n}{24}\right)-\frac{8283562}{1962225} a_{1}(n) \\
& +\frac{832268}{7695} a_{1}\left(\frac{n}{2}\right)+\frac{988401}{8075} a_{1}\left(\frac{n}{3}\right)-\frac{362533312}{654075} a_{1}\left(\frac{n}{4}\right)+\frac{292668}{95} a_{1}\left(\frac{n}{6}\right)+\frac{372644928}{8075} a_{1}\left(\frac{n}{12}\right) \\
& -\frac{1586137}{1051650} \Delta_{3,8}(n)-\frac{14965261}{525825} \Delta_{3,8}\left(\frac{n}{2}\right)+\frac{2129080184}{175275} \Delta_{3,8}\left(\frac{n}{4}\right)+\frac{1967638144}{175275} \Delta_{3,8}\left(\frac{n}{8}\right) \\
& +\frac{132268}{23085} a_{4}(n)-\frac{193504}{1539} a_{4}\left(\frac{n}{2}\right)-\frac{3819008}{405} a_{4}\left(\frac{n}{4}\right)-8 a_{2}(n)-\frac{5040}{19} a_{2}\left(\frac{n}{3}\right)+8 a_{3}(n) \\
& +\frac{3900}{19} a_{3}\left(\frac{n}{3}\right)+\frac{166976}{57} a_{5}(n)+\frac{5469952}{57} a_{5}\left(\frac{n}{2}\right)-8 a_{6}(n)+\frac{70080}{19} a_{6}\left(\frac{n}{2}\right)+\frac{38336}{57} a_{7}(n) \\
& +\frac{90752}{19} a_{8}(n)-\frac{91520}{19} a_{9}(n) \text {, } \\
& N\left(2^{2}, 3^{12}, 6^{2} ; n\right)=\frac{1}{1672800} \sigma_{7}(n)-\frac{1}{1672800} \sigma_{7}\left(\frac{n}{2}\right)-\frac{547}{557600} \sigma_{7}\left(\frac{n}{3}\right)+\frac{1}{418200} \sigma_{7}\left(\frac{n}{4}\right) \\
& +\frac{547}{557600} \sigma_{7}\left(\frac{n}{6}\right)-\frac{32}{52275} \sigma_{7}\left(\frac{n}{8}\right)-\frac{547}{139400} \sigma_{7}\left(\frac{n}{12}\right)+\frac{17504}{17425} \sigma_{7}\left(\frac{n}{24}\right)-\frac{14939714}{1962225} a_{1}(n) \\
& +\frac{1018528}{4617} a_{1}\left(\frac{n}{2}\right)+\frac{1791672}{8075} a_{1}\left(\frac{n}{3}\right)-\frac{8993472}{8075} a_{1}\left(\frac{n}{4}\right)+\frac{113688}{19} a_{1}\left(\frac{n}{6}\right)+\frac{747907008}{8075} a_{1}\left(\frac{n}{12}\right) \\
& -\frac{1437307}{525825} \Delta_{3,8}(n)+-\frac{30527482}{525825} \Delta_{3,8}\left(\frac{n}{2}\right)+\frac{12589064552}{525825} \Delta_{3,8}\left(\frac{n}{4}\right)+\frac{1313856128}{58425} \Delta_{3,8}\left(\frac{n}{8}\right) \\
& +\frac{238856}{23085} a_{4}(n)-\frac{1119904}{4617} a_{4}\left(\frac{n}{2}\right)-\frac{280064}{15} a_{4}\left(\frac{n}{4}\right)-16 a_{2}(n)-\frac{10608}{19} a_{2}\left(\frac{n}{3}\right)+16 a_{3}(n) \\
& +\frac{9240}{19} a_{3}\left(\frac{n}{3}\right)+\frac{309568}{57} a_{5}(n)+\frac{3604864}{19} a_{5}\left(\frac{n}{2}\right)-16 a_{6}(n)+\frac{138816}{19} a_{6}\left(\frac{n}{2}\right)+\frac{85888}{57} a_{7}(n) \\
& +\frac{178944}{19} a_{8}(n)-\frac{187648}{19} a_{9}(n) \text {, } \\
& N\left(2^{2}, 3^{14} ; n\right)=\frac{1}{836400} \sigma_{7}(n)-\frac{1}{836400} \sigma_{7}\left(\frac{n}{2}\right)-\frac{547}{278800} \sigma_{7}\left(\frac{n}{3}\right)+\frac{1}{418200} \sigma_{7}\left(\frac{n}{4}\right) \\
& +\frac{547}{278800} \sigma_{7}\left(\frac{n}{6}\right)-\frac{32}{52275} \sigma_{7}\left(\frac{n}{8}\right)-\frac{547}{139400} \sigma_{7}\left(\frac{n}{12}\right)+\frac{17504}{17425} \sigma_{7}\left(\frac{n}{24}\right)-\frac{21952838}{1962225} a_{1}(n) \\
& +\frac{12667768}{23085} a_{1}\left(\frac{n}{2}\right)+\frac{2947374}{8075} a_{1}\left(\frac{n}{3}\right)-\frac{1907645632}{654075} a_{1}\left(\frac{n}{4}\right)+\frac{1186896}{95} a_{1}\left(\frac{n}{6}\right) \\
& +\frac{1369372608}{8075} a_{1}\left(\frac{n}{12}\right)-\frac{2363344}{525825} \Delta_{3,8}(n)-\frac{4688252}{19475} \Delta_{3,8}\left(\frac{n}{2}\right)+\frac{27425449192}{525825} \Delta_{3,8}\left(\frac{n}{4}\right) \\
& +\frac{10006681984}{175275} \Delta_{3,8}\left(\frac{n}{8}\right)+\frac{454352}{23085} a_{4}(n)-\frac{1936544}{4617} a_{4}\left(\frac{n}{2}\right)-\frac{16890368}{405} a_{4}\left(\frac{n}{4}\right)-28 a_{2}(n) \\
& -\frac{17772}{19} a_{2}\left(\frac{n}{3}\right)+24 a_{3}(n)+\frac{16176}{19} a_{3}\left(\frac{n}{3}\right)+\frac{506272}{57} a_{5}(n)+\frac{24172672}{57} a_{5}\left(\frac{n}{2}\right)-64 a_{6}(n) \\
& +\frac{307264}{19} a_{6}\left(\frac{n}{2}\right)+\frac{258688}{57} a_{7}(n)+\frac{386304}{19} a_{8}(n)-\frac{456448}{19} a_{9}(n) \text {, }
\end{aligned}
$$




$$
\begin{aligned}
& N\left(2^{4}, 3^{2}, 6^{10} ; n\right)=\frac{1}{26764800} \sigma_{7}(n)-\frac{1}{26764800} \sigma_{7}\left(\frac{n}{2}\right)+\frac{273}{8921600} \sigma_{7}\left(\frac{n}{3}\right)-\frac{1}{209100} \sigma_{7}\left(\frac{n}{4}\right) \\
& -\frac{273}{8921600} \sigma_{7}\left(\frac{n}{6}\right)+\frac{64}{52275} \sigma_{7}\left(\frac{n}{8}\right)-\frac{273}{69700} \sigma_{7}\left(\frac{n}{12}\right)+\frac{17472}{17425} \sigma_{7}\left(\frac{n}{24}\right)+\frac{27875381}{31395600} a_{1}(n) \\
& -\frac{316207}{23085} a_{1}\left(\frac{n}{2}\right)-\frac{2854263}{129200} a_{1}\left(\frac{n}{3}\right)+\frac{43510784}{654075} a_{1}\left(\frac{n}{4}\right)-\frac{66579}{95} a_{1}\left(\frac{n}{6}\right) \\
& -\frac{29114496}{8075} a_{1}\left(\frac{n}{12}\right)+\frac{1146539}{4206600} \Delta_{3,8}(n)+\frac{8765039}{701100} \Delta_{3,8}\left(\frac{n}{2}\right)-\frac{1054441684}{525825} \Delta_{3,8}\left(\frac{n}{4}\right) \\
& -\frac{251915008}{175275} \Delta_{3,8}\left(\frac{n}{8}\right)-\frac{26789}{23085} a_{4}(n)+\frac{102800}{4617} a_{4}\left(\frac{n}{2}\right)+\frac{603136}{405} a_{4}\left(\frac{n}{4}\right)+a_{2}(n)+\frac{162}{19} a_{2}\left(\frac{n}{3}\right) \\
& -a_{3}(n)+\frac{522}{19} a_{3}\left(\frac{n}{3}\right)-\frac{8654}{19} a_{5}(n)-\frac{296960}{19} a_{5}\left(\frac{n}{2}\right)+3 a_{6}(n)-\frac{11264}{19} a_{6}\left(\frac{n}{2}\right) \\
& +\frac{544}{57} a_{7}(n)-\frac{11264}{19} a_{8}(n)+\frac{7936}{19} a_{9}(n) \text {, } \\
& N\left(2^{4}, 3^{4}, 6^{8} ; n\right)=\frac{1}{13382400} \sigma_{7}(n)-\frac{1}{13382400} \sigma_{7}\left(\frac{n}{2}\right)+\frac{273}{4460800} \sigma_{7}\left(\frac{n}{3}\right)-\frac{1}{209100} \sigma_{7}\left(\frac{n}{4}\right) \\
& -\frac{273}{4460800} \sigma_{7}\left(\frac{n}{6}\right)+\frac{64}{52275} \sigma_{7}\left(\frac{n}{8}\right)-\frac{273}{69700} \sigma_{7}\left(\frac{n}{12}\right)+\frac{17472}{17425} \sigma_{7}\left(\frac{n}{24}\right)+\frac{27875381}{15697800} a_{1}(n) \\
& -\frac{546686}{23085} a_{1}\left(\frac{n}{2}\right)-\frac{2854263}{64600} a_{1}\left(\frac{n}{3}\right)+\frac{79708544}{654075} a_{1}\left(\frac{n}{4}\right)-\frac{116742}{95} a_{1}\left(\frac{n}{6}\right) \\
& -\frac{69457536}{8075} a_{1}\left(\frac{n}{12}\right)+\frac{1146539}{2103300} \Delta_{3,8}(n)+\frac{2568533}{116850} \Delta_{3,8}\left(\frac{n}{2}\right)-\frac{1879910264}{525825} \Delta_{3,8}\left(\frac{n}{4}\right) \\
& -\frac{458633728}{175275} \Delta_{3,8}\left(\frac{n}{8}\right)-\frac{53578}{23085} a_{4}(n)+\frac{165472}{4617} a_{4}\left(\frac{n}{2}\right)+\frac{1079296}{405} a_{4}\left(\frac{n}{4}\right)+2 a_{2}(n) \\
& +\frac{324}{19} a_{2}\left(\frac{n}{3}\right)-2 a_{3}(n)+\frac{1044}{19} a_{3}\left(\frac{n}{3}\right)-\frac{17308}{19} a_{5}(n)-\frac{530688}{19} a_{5}\left(\frac{n}{2}\right)+6 a_{6}(n) \\
& -\frac{20096}{19} a_{6}\left(\frac{n}{2}\right)+\frac{1088}{57} a_{7}(n)-\frac{22528}{19} a_{8}(n)+\frac{15872}{19} a_{9}(n) \text {, } \\
& N\left(2^{4}, 3^{6}, 6^{6} ; n\right)=\frac{1}{6691200} \sigma_{7}(n)-\frac{1}{6691200} \sigma_{7}\left(\frac{n}{2}\right)+\frac{273}{2230400} \sigma_{7}\left(\frac{n}{3}\right)-\frac{1}{209100} \sigma_{7}\left(\frac{n}{4}\right) \\
& -\frac{273}{2230400} \sigma_{7}\left(\frac{n}{6}\right)+\frac{64}{52275} \sigma_{7}\left(\frac{n}{8}\right)-\frac{273}{69700} \sigma_{7}\left(\frac{n}{12}\right)+\frac{17472}{17425} \sigma_{7}\left(\frac{n}{24}\right)+\frac{24853801}{7848900} a_{1}(n) \\
& -\frac{1075376}{23085} a_{1}\left(\frac{n}{2}\right)-\frac{2469723}{32300} a_{1}\left(\frac{n}{3}\right)+\frac{156782464}{654075} a_{1}\left(\frac{n}{4}\right)-\frac{195312}{95} a_{1}\left(\frac{n}{6}\right) \\
& -\frac{154060416}{8075} a_{1}\left(\frac{n}{12}\right)+\frac{991969}{1051650} \Delta_{3,8}(n)+\frac{2459063}{58425} \Delta_{3,8}\left(\frac{n}{2}\right)-\frac{3446270984}{525825} \Delta_{3,8}\left(\frac{n}{4}\right) \\
& -\frac{889914368}{175275} \Delta_{3,8}\left(\frac{n}{8}\right)-\frac{94876}{23085} a_{4}(n)+\frac{264352}{4617} a_{4}\left(\frac{n}{2}\right)+\frac{2011136}{405} a_{4}\left(\frac{n}{4}\right)+4 a_{2}(n) \\
& \frac{1278}{19} a_{2}\left(\frac{n}{3}\right)-4 a_{3}(n)+\frac{774}{19} a_{3}\left(\frac{n}{3}\right)-\frac{30680}{19} a_{5}(n)-\frac{980224}{19} a_{5}\left(\frac{n}{2}\right)+12 a_{6}(n) \\
& -\frac{37248}{19} a_{6}\left(\frac{n}{2}\right)-\frac{4288}{57} a_{7}(n)-\frac{44544}{19} a_{8}(n)+\frac{35584}{19} a_{9}(n) \text {, }
\end{aligned}
$$




$$
\begin{aligned}
& N\left(2^{4}, 3^{8}, 6^{4} ; n\right)=\frac{1}{3345600} \sigma_{7}(n)-\frac{1}{3345600} \sigma_{7}\left(\frac{n}{2}\right)+\frac{273}{1115200} \sigma_{7}\left(\frac{n}{3}\right)-\frac{1}{209100} \sigma_{7}\left(\frac{n}{4}\right) \\
& -\frac{273}{1115200} \sigma_{7}\left(\frac{n}{6}\right)+\frac{64}{52275} \sigma_{7}\left(\frac{n}{8}\right)-\frac{273}{69700} \sigma_{7}\left(\frac{n}{12}\right)+\frac{17472}{17425} \sigma_{7}\left(\frac{n}{24}\right)+\frac{7277407}{1308150} a_{1}(n) \\
& -\frac{732736}{7695} a_{1}\left(\frac{n}{2}\right)-\frac{2085183}{16150} a_{1}\left(\frac{n}{3}\right)+\frac{106718848}{218025} a_{1}\left(\frac{n}{4}\right)-\frac{337536}{95} a_{1}\left(\frac{n}{6}\right)-\frac{330903936}{8075} a_{1}\left(\frac{n}{12}\right) \\
& +\frac{279133}{175275} \Delta_{3,8}(n)+\frac{1607942}{19475} \Delta_{3,8}\left(\frac{n}{2}\right)-\frac{2164119808}{175275} \Delta_{3,8}\left(\frac{n}{4}\right)-\frac{596421376}{58425} \Delta_{3,8}\left(\frac{n}{8}\right) \\
& -\frac{55064}{7695} a_{4}(n)+\frac{146432}{1539} a_{4}\left(\frac{n}{2}\right)+\frac{1283072}{135} a_{4}\left(\frac{n}{4}\right)+8 a_{2}(n)+\frac{3816}{19} a_{2}\left(\frac{n}{3}\right)-8 a_{3}(n) \\
& -\frac{1080}{19} a_{3}\left(\frac{n}{3}\right)-\frac{53488}{19} a_{5}(n)-\frac{1861376}{19} a_{5}\left(\frac{n}{2}\right)+24 a_{6}(n)-\frac{71040}{19} a_{6}\left(\frac{n}{2}\right)-\frac{7168}{19} a_{7}(n) \\
& -\frac{88064}{19} a_{8}(n)+\frac{78848}{19} a_{9}(n) \text {, } \\
& N\left(2^{4}, 3^{10}, 6^{2} ; n\right)=\frac{1}{1672800} \sigma_{7}(n)-\frac{1}{1672800} \sigma_{7}\left(\frac{n}{2}\right)+\frac{273}{557600} \sigma_{7}\left(\frac{n}{3}\right)-\frac{1}{209100} \sigma_{7}\left(\frac{n}{4}\right) \\
& -\frac{273}{557600} \sigma_{7}\left(\frac{n}{6}\right)+\frac{64}{52275} \sigma_{7}\left(\frac{n}{8}\right)-\frac{273}{69700} \sigma_{7}\left(\frac{n}{12}\right)+\frac{17472}{17425} \sigma_{7}\left(\frac{n}{24}\right)+\frac{19332796}{1962225} a_{1}(n) \\
& -\frac{4611944}{23085} a_{1}\left(\frac{n}{2}\right)-\frac{1811058}{8075} a_{1}\left(\frac{n}{3}\right)+\frac{226137728}{218025} a_{1}\left(\frac{n}{4}\right)-\frac{621768}{95} a_{1}\left(\frac{n}{6}\right)-\frac{700845696}{8075} a_{1}\left(\frac{n}{12}\right) \\
& +\frac{1454423}{525825} \Delta_{3,8}(n)+\frac{28667026}{175275} \Delta_{3,8}\left(\frac{n}{2}\right)-\frac{12639600944}{525825} \Delta_{3,8}\left(\frac{n}{4}\right)-\frac{414534912}{19475} \Delta_{3,8}\left(\frac{n}{8}\right) \\
& -\frac{291304}{23085} a_{4}(n)+\frac{778432}{4617} a_{4}\left(\frac{n}{2}\right)+\frac{2532352}{135} a_{4}\left(\frac{n}{4}\right)+16 a_{2}(n)+\frac{9540}{19} a_{2}\left(\frac{n}{3}\right)-16 a_{3}(n) \\
& -\frac{6120}{19} a_{3}\left(\frac{n}{3}\right)-\frac{94656}{19} a_{5}(n)-\frac{3649024}{19} a_{5}\left(\frac{n}{2}\right)+48 a_{6}(n)-\frac{139904}{19} a_{6}\left(\frac{n}{2}\right)-\frac{65920}{57} a_{7}(n) \\
& -\frac{176384}{19} a_{8}(n)+\frac{172800}{19} a_{9}(n) \text {, } \\
& N\left(2^{4}, 3^{12} ; n\right)=\frac{1}{836400} \sigma_{7}(n)-\frac{1}{836400} \sigma_{7}\left(\frac{n}{2}\right)+\frac{273}{278800} \sigma_{7}\left(\frac{n}{3}\right)-\frac{1}{209100} \sigma_{7}\left(\frac{n}{4}\right) \\
& -\frac{273}{278800} \sigma_{7}\left(\frac{n}{6}\right)+\frac{64}{52275} \sigma_{7}\left(\frac{n}{8}\right)-\frac{273}{69700} \sigma_{7}\left(\frac{n}{12}\right)+\frac{17472}{17425} \sigma_{7}\left(\frac{n}{24}\right)+\frac{34711052}{1962225} a_{1}(n) \\
& -\frac{1909376}{4617} a_{1}\left(\frac{n}{2}\right)-\frac{3294696}{8075} a_{1}\left(\frac{n}{3}\right)+\frac{17986944}{8075} a_{1}\left(\frac{n}{4}\right)-\frac{239232}{19} a_{1}\left(\frac{n}{6}\right)-\frac{1496543616}{8075} a_{1}\left(\frac{n}{12}\right) \\
& +\frac{2645626}{525825} \Delta_{3,8}(n)+\frac{56769892}{175275} \Delta_{3,8}\left(\frac{n}{2}\right)-\frac{24849079504}{525825} \Delta_{3,8}\left(\frac{n}{4}\right)-\frac{2627712256}{58425} \Delta_{3,8}\left(\frac{n}{8}\right) \\
& -\frac{524528}{23085} a_{4}(n)+\frac{1422656}{4617} a_{4}\left(\frac{n}{2}\right)+\frac{560128}{15} a_{4}\left(\frac{n}{4}\right)+32 a_{2}(n)+\frac{21672}{19} a_{2}\left(\frac{n}{3}\right)-32 a_{3}(n) \\
& -\frac{17568}{19} a_{3}\left(\frac{n}{3}\right)-\frac{171520}{19} a_{5}(n)-\frac{7209728}{19} a_{5}\left(\frac{n}{2}\right)+96 a_{6}(n)-\frac{277632}{19} a_{6}\left(\frac{n}{2}\right)-\frac{171776}{57} a_{7}(n) \\
& -\frac{357888}{19} a_{8}(n)+\frac{375296}{19} a_{9}(n) \text {, }
\end{aligned}
$$




$$
\begin{aligned}
& N\left(2^{6}, 3^{2}, 6^{8} ; n\right)=\frac{7}{53529600} \sigma_{7}(n)-\frac{7}{53529600} \sigma_{7}\left(\frac{n}{2}\right)-\frac{549}{17843200} \sigma_{7}\left(\frac{n}{3}\right)+\frac{7}{418200} \sigma_{7}\left(\frac{n}{4}\right) \\
& +\frac{549}{17843200} \sigma_{7}\left(\frac{n}{6}\right)-\frac{224}{52275} \sigma_{7}\left(\frac{n}{8}\right)-\frac{549}{139400} \sigma_{7}\left(\frac{n}{12}\right)+\frac{17568}{17425} \sigma_{7}\left(\frac{n}{24}\right)-\frac{15567697}{6976800} a_{1}(n) \\
& +\frac{101681}{15390} a_{1}\left(\frac{n}{2}\right)+\frac{14187879}{258400} a_{1}\left(\frac{n}{3}\right)-\frac{47241344}{654075} a_{1}\left(\frac{n}{4}\right)+\frac{141201}{190} a_{1}\left(\frac{n}{6}\right)+\frac{67268736}{8075} a_{1}\left(\frac{n}{12}\right) \\
& -\frac{633493}{934800} \Delta_{3,8}(n)-\frac{27091157}{1402200} \Delta_{3,8}\left(\frac{n}{2}\right)+\frac{137626406}{58425} \Delta_{3,8}\left(\frac{n}{4}\right)+\frac{278969728}{175275} \Delta_{3,8}\left(\frac{n}{8}\right) \\
& +\frac{14923}{5130} a_{4}(n)-\frac{18728}{1539} a_{4}\left(\frac{n}{2}\right)-\frac{702976}{405} a_{4}\left(\frac{n}{4}\right)-\frac{3}{2} a_{2}(n)+\frac{891}{38} a_{2}\left(\frac{n}{3}\right)+\frac{3}{2} a_{3}(n) \\
& -\frac{4995}{38} a_{3}\left(\frac{n}{3}\right)+\frac{19911}{19} a_{5}(n)+\frac{348288}{19} a_{5}\left(\frac{n}{2}\right)-\frac{15}{2} a_{6}(n)+\frac{12800}{19} a_{6}\left(\frac{n}{2}\right)-\frac{2896}{19} a_{7}(n) \\
& +\frac{20096}{19} a_{8}(n)-\frac{8576}{19} a_{9}(n) \\
& N\left(2^{6}, 3^{4}, 6^{6} ; n\right)=\frac{7}{26764800} \sigma_{7}(n)-\frac{7}{26764800} \sigma_{7}\left(\frac{n}{2}\right)-\frac{549}{8921600} \sigma_{7}\left(\frac{n}{3}\right)+\frac{7}{418200} \sigma_{7}\left(\frac{n}{4}\right) \\
& +\frac{549}{8921600} \sigma_{7}\left(\frac{n}{6}\right)-\frac{224}{52275} \sigma_{7}\left(\frac{n}{8}\right)-\frac{549}{139400} \sigma_{7}\left(\frac{n}{12}\right)+\frac{17568}{17425} \sigma_{7}\left(\frac{n}{24}\right)-\frac{15567697}{3488400} a_{1}(n) \\
& +\frac{153817}{7695} a_{1}\left(\frac{n}{2}\right)+\frac{14187879}{129200} a_{1}\left(\frac{n}{3}\right)-\frac{105683264}{654075} a_{1}\left(\frac{n}{4}\right)+\frac{171297}{95} a_{1}\left(\frac{n}{6}\right)+\frac{124698816}{8075} a_{1}\left(\frac{n}{12}\right) \\
& -\frac{633493}{467400} \Delta_{3,8}(n)-\frac{28898437}{701100} \Delta_{3,8}\left(\frac{n}{2}\right)+\frac{104093692}{19475} \Delta_{3,8}\left(\frac{n}{4}\right)+\frac{625101568}{175275} \Delta_{3,8}\left(\frac{n}{8}\right) \\
& +\frac{14923}{2565} a_{4}(n)-\frac{62384}{1539} a_{4}\left(\frac{n}{2}\right)-\frac{1586176}{405} a_{4}\left(\frac{n}{4}\right)-3 a_{2}(n)+\frac{891}{19} a_{2}\left(\frac{n}{3}\right)+3 a_{3}(n)-\frac{4995}{19} a_{3}\left(\frac{n}{3}\right) \\
& +\frac{39822}{19} a_{5}(n)+\frac{776832}{19} a_{5}\left(\frac{n}{2}\right)-15 a_{6}(n)+\frac{29248}{19} a_{6}\left(\frac{n}{2}\right)-\frac{5792}{19} a_{7}(n)+\frac{40192}{19} a_{8}(n) \\
& -\frac{17152}{19} a_{9}(n) \\
& N\left(2^{6}, 3^{6}, 6^{4} ; n\right)=\frac{7}{13382400} \sigma_{7}(n)-\frac{7}{13382400} \sigma_{7}\left(\frac{n}{2}\right)-\frac{549}{4460800} \sigma_{7}\left(\frac{n}{3}\right)+\frac{7}{418200} \sigma_{7}\left(\frac{n}{4}\right) \\
& +\frac{549}{4460800} \sigma_{7}\left(\frac{n}{6}\right)-\frac{224}{52275} \sigma_{7}\left(\frac{n}{8}\right)-\frac{549}{139400} \sigma_{7}\left(\frac{n}{12}\right)+\frac{17568}{17425} \sigma_{7}\left(\frac{n}{24}\right)-\frac{39774911}{5232600} a_{1}(n) \\
& +\frac{386272}{7695} a_{1}\left(\frac{n}{2}\right)+\frac{11473659}{64600} a_{1}\left(\frac{n}{3}\right)-\frac{233088064}{654075} a_{1}\left(\frac{n}{4}\right)+\frac{312552}{95} a_{1}\left(\frac{n}{6}\right)+\frac{247588416}{8075} a_{1}\left(\frac{n}{12}\right) \\
& -\frac{1536809}{701100} \Delta_{3,8}(n)-\frac{31625347}{350550} \Delta_{3,8}\left(\frac{n}{2}\right)+\frac{1827576428}{175275} \Delta_{3,8}\left(\frac{n}{4}\right)+\frac{1341978368}{175275} \Delta_{3,8}\left(\frac{n}{8}\right) \\
& +\frac{75358}{7695} a_{4}(n)-\frac{107120}{1539} a_{4}\left(\frac{n}{2}\right)-\frac{3163136}{405} a_{4}\left(\frac{n}{4}\right)-6 a_{2}(n)-\frac{108}{19} a_{2}\left(\frac{n}{3}\right)+6 a_{3}(n) \\
& -\frac{6048}{19} a_{3}\left(\frac{n}{3}\right)+\frac{65100}{19} a_{5}(n)+\frac{1543680}{19} a_{5}\left(\frac{n}{2}\right)-30 a_{6}(n)+\frac{58176}{19} a_{6}\left(\frac{n}{2}\right)-\frac{5728}{19} a_{7}(n) \\
& +\frac{76416}{19} a_{8}(n)-\frac{43392}{19} a_{9}(n) \text {, }
\end{aligned}
$$




$$
\begin{aligned}
& N\left(2^{6}, 3^{8}, 6^{2} ; n\right)=\frac{7}{6691200} \sigma_{7}(n)-\frac{7}{6691200} \sigma_{7}\left(\frac{n}{2}\right)-\frac{549}{2230400} \sigma_{7}\left(\frac{n}{3}\right)+\frac{7}{418200} \sigma_{7}\left(\frac{n}{4}\right) \\
& +\frac{549}{2230400} \sigma_{7}\left(\frac{n}{6}\right)-\frac{224}{52275} \sigma_{7}\left(\frac{n}{8}\right)-\frac{549}{139400} \sigma_{7}\left(\frac{n}{12}\right)+\frac{17568}{17425} \sigma_{7}\left(\frac{n}{24}\right)-\frac{32846731}{2616300} a_{1}(n) \\
& +\frac{897368}{7695} a_{1}\left(\frac{n}{2}\right)+\frac{8759439}{32300} a_{1}\left(\frac{n}{3}\right)-\frac{163314368}{218025} a_{1}\left(\frac{n}{4}\right)+\frac{556128}{95} a_{1}\left(\frac{n}{6}\right)+\frac{508838976}{8075} a_{1}\left(\frac{n}{12}\right) \\
& -\frac{1173139}{350550} \Delta_{3,8}(n)-\frac{3716513}{19475} \Delta_{3,8}\left(\frac{n}{2}\right)+\frac{3447267488}{175275} \Delta_{3,8}\left(\frac{n}{4}\right)+\frac{920319616}{58425} \Delta_{3,8}\left(\frac{n}{8}\right) \\
& +\frac{122356}{7695} a_{4}(n)-\frac{161920}{1539} a_{4}\left(\frac{n}{2}\right)-\frac{2033152}{135} a_{4}\left(\frac{n}{4}\right)-12 a_{2}(n)-\frac{3996}{19} a_{2}\left(\frac{n}{3}\right)+12 a_{3}(n) \\
& -\frac{4212}{19} a_{3}\left(\frac{n}{3}\right)+\frac{101112}{19} a_{5}(n)+\frac{2957952}{19} a_{5}\left(\frac{n}{2}\right)-60 a_{6}(n)+\frac{112064}{19} a_{6}\left(\frac{n}{2}\right)+\frac{256}{19} a_{7}(n) \\
& +\frac{144896}{19} a_{8}(n)-\frac{104960}{19} a(n) \text {, } \\
& N\left(2^{6}, 3^{10} ; n\right)=\frac{7}{3345600} \sigma_{7}(n)-\frac{7}{3345600} \sigma_{7}\left(\frac{n}{2}\right)-\frac{549}{1115200} \sigma_{7}\left(\frac{n}{3}\right)+\frac{7}{418200} \sigma_{7}\left(\frac{n}{4}\right) \\
& +\frac{549}{1115200} \sigma_{7}\left(\frac{n}{6}\right)-\frac{224}{52275} \sigma_{7}\left(\frac{n}{8}\right)-\frac{549}{139400} \sigma_{7}\left(\frac{n}{12}\right)+\frac{17568}{17425} \sigma_{7}\left(\frac{n}{24}\right)-\frac{13425458}{654075} a_{1}(n) \\
& +\frac{404948}{1539} a_{1}\left(\frac{n}{2}\right)+\frac{3435327}{8075} a_{1}\left(\frac{n}{3}\right)-\frac{342442688}{218025} a_{1}\left(\frac{n}{4}\right)+\frac{208260}{19} a_{1}\left(\frac{n}{6}\right)+\frac{1063751616}{8075} a_{1}\left(\frac{n}{12}\right) \\
& -\frac{1840133}{350550} \Delta_{3,8}(n)-\frac{7648481}{19475} \Delta_{3,8}\left(\frac{n}{2}\right)+\frac{6479819368}{175275} \Delta_{3,8}\left(\frac{n}{4}\right)+\frac{1891094656}{58425} \Delta_{3,8}\left(\frac{n}{8}\right) \\
& +\frac{198332}{7695} a_{4}(n)-\frac{235424}{1539} a_{4}\left(\frac{n}{2}\right)-\frac{3907072}{135} a_{4}\left(\frac{n}{4}\right)-24 a_{2}(n)-\frac{13608}{19} a_{2}\left(\frac{n}{3}\right)+24 a_{3}(n) \\
& +\frac{3348}{19} a_{3}\left(\frac{n}{3}\right)+\frac{152832}{19} a_{5}(n)+\frac{5639424}{19} a_{5}\left(\frac{n}{2}\right)-120 a_{6}(n)+\frac{215360}{19} a_{6}\left(\frac{n}{2}\right)+\frac{22464}{19} a_{7}(n) \\
& +\frac{277376}{19} a_{8}(n)-\frac{245888}{19} a_{9}(n) \text {, } \\
& N\left(2^{8}, 3^{2}, 6^{6} ; n\right)=\frac{1}{2676480} \sigma_{7}(n)-\frac{1}{2676480} \sigma_{7}\left(\frac{n}{2}\right)+\frac{27}{892160} \sigma_{7}\left(\frac{n}{3}\right)-\frac{1}{20910} \sigma_{7}\left(\frac{n}{4}\right) \\
& -\frac{27}{892160} \sigma_{7}\left(\frac{n}{6}\right)+\frac{128}{10455} \sigma_{7}\left(\frac{n}{8}\right)-\frac{27}{6970} \sigma_{7}\left(\frac{n}{12}\right)+\frac{3456}{3485} \sigma_{7}\left(\frac{n}{24}\right)+\frac{605447}{116280} a_{1}(n) \\
& -\frac{35158}{2565} a_{1}\left(\frac{n}{2}\right)-\frac{1735857}{12920} a_{1}\left(\frac{n}{3}\right)+\frac{1071104}{8721} a_{1}\left(\frac{n}{4}\right)-\frac{251964}{95} a_{1}\left(\frac{n}{6}\right)-\frac{1558656}{323} a_{1}\left(\frac{n}{12}\right) \\
& +\frac{77519}{46740} \Delta_{3,8}(n)+\frac{343787}{7790} \Delta_{3,8}\left(\frac{n}{2}\right)-\frac{70391368}{11685} \Delta_{3,8}\left(\frac{n}{4}\right)-\frac{7043584}{2337} \Delta_{3,8}\left(\frac{n}{8}\right) \\
& -\frac{1174}{171} a_{4}(n)+\frac{39968}{513} a_{4}\left(\frac{n}{2}\right)+\frac{112640}{27} a_{4}\left(\frac{n}{4}\right)+2 a_{2}(n)-\frac{3321}{19} a_{2}\left(\frac{n}{3}\right)-2 a_{3}(n) \\
& +\frac{9477}{19} a_{3}\left(\frac{n}{3}\right)-\frac{45900}{19} a_{5}(n)-\frac{838656}{19} a_{5}\left(\frac{n}{2}\right)+14 a_{6}(n)-\frac{31744}{19} a_{6}\left(\frac{n}{2}\right)+\frac{14016}{19} a_{7}(n) \\
& -\frac{31744}{19} a_{8}(n)-\frac{4608}{19} a_{9}(n) \text {, }
\end{aligned}
$$




$$
\begin{aligned}
& N\left(2^{8}, 3^{4}, 6^{4} ; n\right)=\frac{1}{1338240} \sigma_{7}(n)-\frac{1}{1338240} \sigma_{7}\left(\frac{n}{2}\right)+\frac{27}{446080} \sigma_{7}\left(\frac{n}{3}\right)-\frac{1}{20910} \sigma_{7}\left(\frac{n}{4}\right) \\
& -\frac{27}{446080} \sigma_{7}\left(\frac{n}{6}\right)+\frac{128}{10455} \sigma_{7}\left(\frac{n}{8}\right)-\frac{27}{6970} \sigma_{7}\left(\frac{n}{12}\right)+\frac{3456}{3485} \sigma_{7}\left(\frac{n}{24}\right)+\frac{605447}{58140} a_{1}(n) \\
& -\frac{14116}{855} a_{1}\left(\frac{n}{2}\right)-\frac{1735857}{6460} a_{1}\left(\frac{n}{3}\right)+\frac{712448}{2907} a_{1}\left(\frac{n}{4}\right)-\frac{479304}{95} a_{1}\left(\frac{n}{6}\right)-\frac{3379968}{323} a_{1}\left(\frac{n}{12}\right) \\
& +\frac{77519}{23370} \Delta_{3,8}(n)+\frac{331323}{3895} \Delta_{3,8}\left(\frac{n}{2}\right)-\frac{130981168}{11685} \Delta_{3,8}\left(\frac{n}{4}\right)-\frac{4566016}{779} \Delta_{3,8}\left(\frac{n}{8}\right) \\
& -\frac{2348}{171} a_{4}(n)+\frac{22592}{171} a_{4}\left(\frac{n}{2}\right)+\frac{69632}{9} a_{4}\left(\frac{n}{4}\right)+4 a_{2}(n)-\frac{6642}{19} a_{2}\left(\frac{n}{3}\right)-4 a_{3}(n) \\
& +\frac{18954}{19} a_{3}\left(\frac{n}{3}\right)-\frac{91800}{19} a_{5}(n)-\frac{1589760}{19} a_{5}\left(\frac{n}{2}\right)+28 a_{6}(n)-\frac{58624}{19} a_{6}\left(\frac{n}{2}\right) \\
& +\frac{28032}{19} a_{7}(n)-\frac{63488}{19} a_{8}(n)-\frac{9216}{19} a_{9}(n) \text {, } \\
& N\left(2^{8}, 3^{6}, 6^{2} ; n\right)=\frac{1}{669120} \sigma_{7}(n)+\frac{27}{223040} \sigma_{7}\left(\frac{n}{2}\right)-\frac{1}{669120} \sigma_{7}\left(\frac{n}{3}\right)-\frac{1}{20910} \sigma_{7}\left(\frac{n}{4}\right) \\
& -\frac{27}{223040} \sigma_{7}\left(\frac{n}{6}\right)+\frac{128}{10455} \sigma_{7}\left(\frac{n}{8}\right)-\frac{27}{6970} \sigma_{7}\left(\frac{n}{12}\right)+\frac{3456}{3485} \sigma_{7}\left(\frac{n}{24}\right)+\frac{301157}{17442} a_{1}(n) \\
& -\frac{5536}{171} a_{1}\left(\frac{n}{2}\right)-\frac{267489}{646} a_{1}\left(\frac{n}{3}\right)+\frac{17458432}{43605} a_{1}\left(\frac{n}{4}\right)-\frac{145584}{19} a_{1}\left(\frac{n}{6}\right)-\frac{39930624}{1615} a_{1}\left(\frac{n}{12}\right) \\
& +\frac{11945}{2337} \Delta_{3,8}(n)+\frac{417694}{2337} \Delta_{3,8}\left(\frac{n}{2}\right)-\frac{72114608}{3895} \Delta_{3,8}\left(\frac{n}{4}\right)-\frac{113035264}{11685} \Delta_{3,8}\left(\frac{n}{8}\right) \\
& -\frac{11480}{513} a_{4}(n)+\frac{28864}{171} a_{4}\left(\frac{n}{2}\right)+\frac{352256}{27} a_{4}\left(\frac{n}{4}\right)+8 a_{2}(n)-\frac{7614}{19} a_{2}\left(\frac{n}{3}\right)-8 a_{3}(n) \\
& +\frac{26082}{19} a_{3}\left(\frac{n}{3}\right)-\frac{142704}{19} a_{5}(n)-\frac{2668032}{19} a_{5}\left(\frac{n}{2}\right)+56 a_{6}(n)-\frac{98048}{19} a_{6}\left(\frac{n}{2}\right) \\
& +\frac{40320}{19} a_{7}(n)-\frac{112640}{19} a_{8}(n)+\frac{1536}{19} a_{9}(n) \text {, } \\
& N\left(2^{8}, 3^{8} ; n\right)=\frac{1}{334560} \sigma_{7}(n)-\frac{1}{334560} \sigma_{7}\left(\frac{n}{2}\right)+\frac{27}{111520} \sigma_{7}\left(\frac{n}{3}\right)-\frac{1}{20910} \sigma_{7}\left(\frac{n}{4}\right) \\
& -\frac{27}{111520} \sigma_{7}\left(\frac{n}{6}\right)+\frac{128}{10455} \sigma_{7}\left(\frac{n}{8}\right)-\frac{27}{6970} \sigma_{7}\left(\frac{n}{12}\right)+\frac{3456}{3485} \sigma_{7}\left(\frac{n}{24}\right)+\frac{1195229}{43605} a_{1}(n) \\
& -\frac{208064}{2565} a_{1}\left(\frac{n}{2}\right)-\frac{939033}{1615} a_{1}\left(\frac{n}{3}\right)+\frac{1774336}{2907} a_{1}\left(\frac{n}{4}\right)-\frac{1084752}{95} a_{1}\left(\frac{n}{6}\right)-\frac{17715456}{323} a_{1}\left(\frac{n}{12}\right) \\
& +\frac{27954}{3895} \Delta_{3,8}(n)+\frac{1442716}{3895} \Delta_{3,8}\left(\frac{n}{2}\right)-\frac{350230144}{11685} \Delta_{3,8}\left(\frac{n}{4}\right)-\frac{35466752}{2337} \Delta_{3,8}\left(\frac{n}{8}\right) \\
& -\frac{17744}{513} a_{4}(n)+\frac{94720}{513} a_{4}\left(\frac{n}{2}\right)+\frac{194560}{9} a_{4}\left(\frac{n}{4}\right)+16 a_{2}(n)-\frac{3888}{19} a_{2}\left(\frac{n}{3}\right)-16 a_{3}(n) \\
& +\frac{28512}{19} a_{3}\left(\frac{n}{3}\right)-\frac{203616}{19} a_{5}(n)-\frac{4400640}{19} a_{5}\left(\frac{n}{2}\right)+112 a_{6}(n)-\frac{162560}{19} a_{6}\left(\frac{n}{2}\right) \\
& +\frac{49152}{19} a_{7}(n)-\frac{196608}{19} a_{8}(n)+\frac{43008}{19} a_{9}(n) \text {, }
\end{aligned}
$$




$$
\begin{aligned}
& N\left(2^{10}, 3^{2}, 6^{4} ; n\right)=\frac{61}{53529600} \sigma_{7}(n)-\frac{61}{53529600} \sigma_{7}\left(\frac{n}{2}\right)-\frac{567}{17843200} \sigma_{7}\left(\frac{n}{3}\right)+\frac{61}{418200} \sigma_{7}\left(\frac{n}{4}\right) \\
& +\frac{567}{17843200} \sigma_{7}\left(\frac{n}{6}\right)-\frac{1952}{52275} \sigma_{7}\left(\frac{n}{8}\right)-\frac{567}{139400} \sigma_{7}\left(\frac{n}{12}\right)+\frac{18144}{17425} \sigma_{7}\left(\frac{n}{24}\right)-\frac{285322073}{20930400} a_{1}(n) \\
& -\frac{219391}{5130} a_{1}\left(\frac{n}{2}\right)+\frac{97787637}{258400} a_{1}\left(\frac{n}{3}\right)-\frac{43532672}{654075} a_{1}\left(\frac{n}{4}\right)+\frac{1081467}{190} a_{1}\left(\frac{n}{6}\right)+\frac{9283968}{8075} a_{1}\left(\frac{n}{12}\right) \\
& -\frac{13101737}{2804400} \Delta_{3,8}(n)-\frac{66256091}{1402200} \Delta_{3,8}\left(\frac{n}{2}\right)+\frac{1694052494}{175275} \Delta_{3,8}\left(\frac{n}{4}\right)+\frac{448987264}{175275} \Delta_{3,8}\left(\frac{n}{8}\right) \\
& +\frac{281687}{15390} a_{4}(n)-\frac{73928}{513} a_{4}\left(\frac{n}{2}\right)-\frac{2395648}{405} a_{4}\left(\frac{n}{4}\right)-\frac{5}{2} a_{2}(n)+\frac{26865}{38} a_{2}\left(\frac{n}{3}\right)+\frac{5}{2} a_{3}(n) \\
& -\frac{63801}{38} a_{3}\left(\frac{n}{3}\right)+\frac{127917}{19} a_{5}(n)+\frac{1315968}{19} a_{5}\left(\frac{n}{2}\right)-\frac{45}{2} a_{6}(n)+\frac{45312}{19} a_{6}\left(\frac{n}{2}\right)-\frac{49328}{19} a_{7}(n) \\
& +\frac{57472}{19} a_{8}(n)+\frac{57728}{19} a_{9}(n) \text {, } \\
& N\left(2^{10}, 3^{4}, 6^{2} ; n\right)=\frac{61}{26764800} \sigma_{7}(n)-\frac{61}{26764800} \sigma_{7}\left(\frac{n}{2}\right)-\frac{567}{8921600} \sigma_{7}\left(\frac{n}{3}\right)+\frac{61}{418200} \sigma_{7}\left(\frac{n}{4}\right) \\
& +\frac{567}{8921600} \sigma_{7}\left(\frac{n}{6}\right)-\frac{1952}{52275} \sigma_{7}\left(\frac{n}{8}\right)-\frac{567}{139400} \sigma_{7}\left(\frac{n}{12}\right)+\frac{18144}{17425} \sigma_{7}\left(\frac{n}{24}\right)-\frac{285322073}{10465200} a_{1}(n) \\
& -\frac{57373}{855} a_{1}\left(\frac{n}{2}\right)+\frac{97787637}{129200} a_{1}\left(\frac{n}{3}\right)-\frac{30634432}{654075} a_{1}\left(\frac{n}{4}\right)+\frac{1056843}{95} a_{1}\left(\frac{n}{6}\right)-\frac{20140992}{8075} a_{1}\left(\frac{n}{12}\right) \\
& -\frac{13101737}{1402200} \Delta_{3,8}(n)-\frac{72425771}{701100} \Delta_{3,8}\left(\frac{n}{2}\right)+\frac{3569401924}{175275} \Delta_{3,8}\left(\frac{n}{4}\right)+\frac{688112384}{175275} \Delta_{3,8}\left(\frac{n}{8}\right) \\
& +\frac{281687}{7695} a_{4}(n)-\frac{54352}{171} a_{4}\left(\frac{n}{2}\right)-\frac{5116928}{405} a_{4}\left(\frac{n}{4}\right)-5 a_{2}(n)+\frac{26865}{19} a_{2}\left(\frac{n}{3}\right)+5 a_{3}(n) \\
& -\frac{63801}{19} a_{3}\left(\frac{n}{3}\right)+\frac{255834}{19} a_{5}(n)+\frac{2697600}{19} a_{5}\left(\frac{n}{2}\right)-45 a_{6}(n)+\frac{96704}{19} a_{6}\left(\frac{n}{2}\right)-\frac{98656}{19} a_{7}(n) \\
& +\frac{114944}{19} a_{8}(n)+\frac{115456}{19} a_{9}(n) \text {, } \\
& N\left(2^{10}, 3^{6} ; n\right)=\frac{61}{13382400} \sigma_{7}(n)-\frac{61}{13382400} \sigma_{7}\left(\frac{n}{2}\right)-\frac{567}{4460800} \sigma_{7}\left(\frac{n}{3}\right)+\frac{61}{418200} \sigma_{7}\left(\frac{n}{4}\right) \\
& +\frac{567}{4460800} \sigma_{7}\left(\frac{n}{6}\right)-\frac{1952}{52275} \sigma_{7}\left(\frac{n}{8}\right)-\frac{567}{139400} \sigma_{7}\left(\frac{n}{12}\right)+\frac{18144}{17425} \sigma_{7}\left(\frac{n}{24}\right)-\frac{234601213}{5232600} a_{1}(n) \\
& -\frac{371312}{2565} a_{1}\left(\frac{n}{2}\right)+\frac{72362097}{64600} a_{1}\left(\frac{n}{3}\right)+\frac{185115968}{654075} a_{1}\left(\frac{n}{4}\right)+\frac{1400904}{95} a_{1}\left(\frac{n}{6}\right) \\
& -\frac{83299392}{8075} a_{1}\left(\frac{n}{12}\right)-\frac{9695047}{701100} \Delta_{3,8}(n)-\frac{86556421}{350550} \Delta_{3,8}\left(\frac{n}{2}\right)+\frac{5289552004}{175275} \Delta_{3,8}\left(\frac{n}{4}\right) \\
& +\frac{18992384}{175275} \Delta_{3,8}\left(\frac{n}{8}\right)+\frac{451394}{7695} a_{4}(n)-\frac{210800}{513} a_{4}\left(\frac{n}{2}\right)-\frac{7482368}{405} a_{4}\left(\frac{n}{4}\right)-10 a_{2}(n) \\
& +\frac{37152}{19} a_{2}\left(\frac{n}{3}\right)+10 a_{3}(n)-\frac{92556}{19} a_{3}\left(\frac{n}{3}\right)+\frac{394884}{19} a_{5}(n)+\frac{4018944}{19} a_{5}\left(\frac{n}{2}\right)-90 a_{6}(n) \\
& +\frac{140224}{19} a_{6}\left(\frac{n}{2}\right)-\frac{161824}{19} a_{7}(n)+\frac{170624}{19} a_{8}(n)+\frac{201088}{19} a_{9}(n),
\end{aligned}
$$




$$
\begin{aligned}
N & \left(2^{12}, 3^{2}, 6^{2} ; n\right)=\frac{91}{26764800} \sigma_{7}(n)-\frac{91}{26764800} \sigma_{7}\left(\frac{n}{2}\right)+\frac{243}{8921600} \sigma_{7}\left(\frac{n}{3}\right)-\frac{91}{209100} \sigma_{7}\left(\frac{n}{4}\right) \\
- & \frac{243}{8921600} \sigma_{7}\left(\frac{n}{6}\right)+\frac{5824}{52275} \sigma_{7}\left(\frac{n}{8}\right)-\frac{243}{69700} \sigma_{7}\left(\frac{n}{12}\right)+\frac{15552}{17425} \sigma_{7}\left(\frac{n}{24}\right)+\frac{389706157}{10465200} a_{1}(n) \\
+ & \frac{238627}{2565} a_{1}\left(\frac{n}{2}\right)-\frac{142817733}{129200} a_{1}\left(\frac{n}{3}\right)-\frac{112946176}{654075} a_{1}\left(\frac{n}{4}\right)-\frac{1627389}{95} a_{1}\left(\frac{n}{6}\right)+\frac{188060544}{8075} a_{1}\left(\frac{n}{12}\right) \\
+ & \frac{19135483}{1402200} \Delta_{3,8}(n)+\frac{16314349}{701100} \Delta_{3,8}\left(\frac{n}{2}\right)-\frac{4824391508}{175275} \Delta_{3,8}\left(\frac{n}{4}\right)-\frac{225196288}{175275} \Delta_{3,8}\left(\frac{n}{8}\right) \\
- & \frac{391573}{7695} a_{4}(n)+\frac{318256}{513} a_{4}\left(\frac{n}{2}\right)+\frac{6470656}{405} a_{4}\left(\frac{n}{4}\right)+3 a_{2}(n)-\frac{46116}{19} a_{2}\left(\frac{n}{3}\right)-3 a_{3}(n) \\
+ & \frac{101520}{19} a_{3}\left(\frac{n}{3}\right)-\frac{374778}{19} a_{5}(n)-\frac{3296256}{19} a_{5}\left(\frac{n}{2}\right)+33 a_{6}(n)-\frac{126976}{19} a_{6}\left(\frac{n}{2}\right)+\frac{155168}{19} a_{7}(n) \\
- & \frac{126976}{19} a_{8}(n)-\frac{217856}{19} a_{9}(n), \\
& N\left(2^{12}, 3^{4} ; n\right)=\frac{91}{13382400} \sigma_{7}(n)-\frac{91}{13382400} \sigma_{7}\left(\frac{n}{2}\right)+\frac{243}{4460800} \sigma_{7}\left(\frac{n}{3}\right)-\frac{91}{209100} \sigma_{7}\left(\frac{n}{4}\right) \\
& -\frac{243}{4460800} \sigma_{7}\left(\frac{n}{6}\right)+\frac{5824}{52275} \sigma_{7}\left(\frac{n}{8}\right)-\frac{243}{69700} \sigma_{7}\left(\frac{n}{12}\right)+\frac{15552}{17425} \sigma_{7}\left(\frac{n}{24}\right)+\frac{389706157}{5232600} a_{1}(n) \\
& +\frac{127918}{513} a_{1}\left(\frac{n}{2}\right)-\frac{142817733}{64600} a_{1}\left(\frac{n}{3}\right)-\frac{24807296}{654075} a_{1}\left(\frac{n}{4}\right)-\frac{591858}{19} a_{1}\left(\frac{n}{6}\right)+\frac{500817024}{8075} a_{1}\left(\frac{n}{12}\right) \\
& +\frac{19135483}{701100} \Delta_{3,8}(n)+\frac{28092829}{350550} \Delta_{3,8}\left(\frac{n}{2}\right)-\frac{9499635448}{175275} \Delta_{3,8}\left(\frac{n}{4}\right)-\frac{1307176448}{175275} \Delta_{3,8}\left(\frac{n}{8}\right) \\
& -\frac{783146}{7695} a_{4}(n)+\frac{574496}{513} a_{4}\left(\frac{n}{2}\right)+\frac{12978176}{405} a_{4}\left(\frac{n}{4}\right)+6 a_{2}(n)-\frac{92232}{19} a_{2}\left(\frac{n}{3}\right)-6 a_{3}(n) \\
& +\frac{203040}{19} a_{3}\left(\frac{n}{3}\right)-\frac{749556}{19} a_{5}(n)-\frac{6986496}{19} a_{5}\left(\frac{n}{2}\right)+66 a_{6}(n)-\frac{246656}{19} a_{6}\left(\frac{n}{2}\right)+\frac{310336}{19} a_{7}(n) \\
& -\frac{253952}{19} a_{8}(n)-\frac{435712}{19} a_{9}(n),
\end{aligned}
$$$$
N\left(2^{14}, 3^{2} ; n\right)=\frac{547}{53529600} \sigma_{7}(n)-\frac{547}{53529600} \sigma_{7}\left(\frac{n}{2}\right)-\frac{729}{17843200} \sigma_{7}\left(\frac{n}{3}\right)+\frac{547}{418200} \sigma_{7}\left(\frac{n}{4}\right)
$$$$
+\frac{729}{17843200} \sigma_{7}\left(\frac{n}{6}\right)-\frac{17504}{52275} \sigma_{7}\left(\frac{n}{8}\right)-\frac{729}{139400} \sigma_{7}\left(\frac{n}{12}\right)+\frac{23328}{17425} \sigma_{7}\left(\frac{n}{24}\right)-\frac{724244357}{6976800} a_{1}(n)
$$$$
-\frac{1724201}{5130} a_{1}\left(\frac{n}{2}\right)+\frac{861519699}{258400} a_{1}\left(\frac{n}{3}\right)-\frac{77762176}{72675} a_{1}\left(\frac{n}{4}\right)+\frac{10262997}{190} a_{1}\left(\frac{n}{6}\right)-\frac{544696704}{8075} a_{1}\left(\frac{n}{12}\right)
$$$$
-\frac{38477233}{934800} \Delta_{3,8}(n)+\frac{69754901}{467400} \Delta_{3,8}\left(\frac{n}{2}\right)+\frac{4634810446}{58425} \Delta_{3,8}\left(\frac{n}{4}\right)+\frac{1683844736}{58425} \Delta_{3,8}\left(\frac{n}{8}\right)
$$$$
+\frac{743663}{5130} a_{4}(n)-\frac{925912}{513} a_{4}\left(\frac{n}{2}\right)-\frac{2077184}{45} a_{4}\left(\frac{n}{4}\right)-\frac{7}{2} a_{2}(n)+\frac{292167}{38} a_{2}\left(\frac{n}{3}\right)+\frac{7}{2} a_{3}(n)
$$$$
-\frac{624591}{38} a_{3}\left(\frac{n}{3}\right)+\frac{1114371}{19} a_{5}(n)+\frac{10415232}{19} a_{5}\left(\frac{n}{2}\right)-\frac{91}{2} a_{6}(n)+\frac{363520}{19} a_{6}\left(\frac{n}{2}\right)
$$$$
-\frac{444432}{19} a_{7}(n)+\frac{380544}{19} a_{8}(n)+\frac{603264}{19} a_{9}(n) \text {, }
$$ 


$$
\begin{aligned}
& N\left(1,2,3,6^{13} ; n\right)=\frac{1}{107059200} \sigma_{7}(n)-\frac{1}{107059200} \sigma_{7}\left(\frac{n}{2}\right) \\
& +\frac{1093}{35686400} \sigma_{7}\left(\frac{n}{3}\right)-\frac{1}{836400} \sigma_{7}\left(\frac{n}{4}\right)-\frac{1093}{35686400} \sigma_{7}\left(\frac{n}{6}\right) \\
& +\frac{16}{52275} \sigma_{7}\left(\frac{n}{8}\right)-\frac{1093}{278800} \sigma_{7}\left(\frac{n}{12}\right)+\frac{17488}{17425} \sigma_{7}\left(\frac{n}{24}\right) \\
& -\frac{94244213}{41860800} a_{1}(n)-\frac{974467}{18468} a_{1}\left(\frac{n}{2}\right)-\frac{17320503}{516800} a_{1}\left(\frac{n}{3}\right) \\
& +\frac{226133056}{654075} a_{1}\left(\frac{n}{4}\right)-\frac{13197}{76} a_{1}\left(\frac{n}{6}\right)+\frac{63457536}{8075} a_{1}\left(\frac{n}{12}\right) \\
& +\frac{2319703}{5608800} \Delta_{3,8}(n)-\frac{397749433}{8413200} \Delta_{3,8}\left(\frac{n}{2}\right)-\frac{1484822891}{525825} \Delta_{3,8}\left(\frac{n}{4}\right) \\
& -\frac{1083399872}{175275} \Delta_{3,8}\left(\frac{n}{8}\right)+\frac{56567}{30780} a_{4}(n)+\frac{250108}{4617} a_{4}\left(\frac{n}{2}\right) \\
& +\frac{1051904}{405} a_{4}\left(\frac{n}{4}\right)-\frac{7}{4} a_{2}(n)-\frac{7347}{76} a_{2}\left(\frac{n}{3}\right)+\frac{15}{4} a_{3}(n) \\
& +\frac{7803}{76} a_{3}\left(\frac{n}{3}\right)-\frac{23893}{114} a_{5}(n)-\frac{512704}{19} a_{5}\left(\frac{n}{2}\right)-\frac{65}{4} a_{6}(n) \\
& -\frac{17984}{19} a_{6}\left(\frac{n}{2}\right)-\frac{38344}{57} a_{7}(n)-\frac{17984}{19} a_{8}(n)+\frac{39616}{19} a_{9}(n), \\
& N\left(1,2,3^{3}, 6^{11} ; n\right)=\frac{1}{53529600} \sigma_{7}(n)-\frac{1}{53529600} \sigma_{7}\left(\frac{n}{2}\right) \\
& +\frac{1093}{17843200} \sigma_{7}\left(\frac{n}{3}\right)-\frac{1}{836400} \sigma_{7}\left(\frac{n}{4}\right)-\frac{1093}{17843200} \sigma_{7}\left(\frac{n}{6}\right) \\
& +\frac{16}{52275} \sigma_{7}\left(\frac{n}{8}\right)-\frac{1093}{278800} \sigma_{7}\left(\frac{n}{12}\right)+\frac{17488}{17425} \sigma_{7}\left(\frac{n}{24}\right) \\
& -\frac{127428629}{62791200} a_{1}(n)-\frac{2568887}{46170} a_{1}\left(\frac{n}{2}\right)-\frac{10090233}{258400} a_{1}\left(\frac{n}{3}\right) \\
& +\frac{236112736}{654075} a_{1}\left(\frac{n}{4}\right)-\frac{62349}{190} a_{1}\left(\frac{n}{6}\right)+\frac{50426016}{8075} a_{1}\left(\frac{n}{12}\right) \\
& +\frac{506603}{1051650} \Delta_{3,8}(n)-\frac{10529371}{233700} \Delta_{3,8}\left(\frac{n}{2}\right)-\frac{1691626276}{525825} \Delta_{3,8}\left(\frac{n}{4}\right) \\
& -\frac{379689344}{58425} \Delta_{3,8}\left(\frac{n}{8}\right)+\frac{35728}{23085} a_{4}(n)+\frac{268208}{4617} a_{4}\left(\frac{n}{2}\right) \\
& +\frac{1170944}{405} a_{4}\left(\frac{n}{4}\right)-\frac{3}{2} a_{2}(n)-\frac{3621}{38} a_{2}\left(\frac{n}{3}\right)+\frac{7}{2} a_{3}(n)+\frac{4305}{38} a_{3}\left(\frac{n}{3}\right) \\
& -\frac{18437}{57} a_{5}(n)-\frac{1708544}{57} a_{5}\left(\frac{n}{2}\right)-\frac{31}{2} a_{6}(n)-\frac{20192}{19} a_{6}\left(\frac{n}{2}\right) \\
& -\frac{12736}{19} a_{7}(n)-\frac{20800}{19} a_{8}(n)+\frac{41600}{19} a_{9}(n) \text {, }
\end{aligned}
$$




$$
\begin{aligned}
& N\left(1,2,3^{5}, 6^{9} ; n\right)=\frac{1}{26764800} \sigma_{7}(n)-\frac{1}{26764800} \sigma_{7}\left(\frac{n}{2}\right)+\frac{1093}{8921600} \sigma_{7}\left(\frac{n}{3}\right)-\frac{1}{836400} \sigma_{7}\left(\frac{n}{4}\right) \\
& -\frac{1093}{8921600} \sigma_{7}\left(\frac{n}{6}\right)+\frac{16}{52275} \sigma_{7}\left(\frac{n}{8}\right)-\frac{1093}{278800} \sigma_{7}\left(\frac{n}{12}\right)+\frac{17488}{17425} \sigma_{7}\left(\frac{n}{24}\right)-\frac{54251789}{31395600} a_{1}(n) \\
& -\frac{1433218}{23085} a_{1}\left(\frac{n}{2}\right)-\frac{6179553}{129200} a_{1}\left(\frac{n}{3}\right)+\frac{86149792}{218025} a_{1}\left(\frac{n}{4}\right)-\frac{52266}{95} a_{1}\left(\frac{n}{6}\right)+\frac{27773856}{8075} a_{1}\left(\frac{n}{12}\right) \\
& +\frac{620221}{1051650} \Delta_{3,8}(n)-\frac{45487259}{1051650} \Delta_{3,8}\left(\frac{n}{2}\right)-\frac{2097692326}{525825} \Delta_{3,8}\left(\frac{n}{4}\right)-\frac{1260874112}{175275} \Delta_{3,8}\left(\frac{n}{8}\right) \\
& +\frac{26276}{23085} a_{4}(n)+\frac{307448}{4617} a_{4}\left(\frac{n}{2}\right)+\frac{470528}{135} a_{4}\left(\frac{n}{4}\right)-a_{2}(n)-\frac{1587}{19} a_{2}\left(\frac{n}{3}\right)+3 a_{3}(n) \\
& +\frac{2157}{19} a_{3}\left(\frac{n}{3}\right)-\frac{9966}{19} a_{5}(n)-\frac{2054272}{57} a_{5}\left(\frac{n}{2}\right)-15 a_{6}(n)-\frac{24608}{19} a_{6}\left(\frac{n}{2}\right)-\frac{39760}{57} a_{7}(n) \\
& -\frac{26432}{19} a_{8}(n)+\frac{46784}{19} a_{9}(n) \text {, } \\
& N\left(1,2,3^{7}, 6^{7} ; n\right)=\frac{1}{13382400} \sigma_{7}(n)-\frac{1}{13382400} \sigma_{7}\left(\frac{n}{2}\right)+\frac{1093}{4460800} \sigma_{7}\left(\frac{n}{3}\right)-\frac{1}{836400} \sigma_{7}\left(\frac{n}{4}\right) \\
& -\frac{1093}{4460800} \sigma_{7}\left(\frac{n}{6}\right)+\frac{16}{52275} \sigma_{7}\left(\frac{n}{8}\right)-\frac{1093}{278800} \sigma_{7}\left(\frac{n}{12}\right)+\frac{17488}{17425} \sigma_{7}\left(\frac{n}{24}\right)-\frac{19174159}{15697800} a_{1}(n) \\
& -\frac{193982}{2565} a_{1}\left(\frac{n}{2}\right)-\frac{4031943}{64600} a_{1}\left(\frac{n}{3}\right)+\frac{303568736}{654075} a_{1}\left(\frac{n}{4}\right)-\frac{89466}{95} a_{1}\left(\frac{n}{6}\right) \\
& -\frac{18199584}{8075} a_{1}\left(\frac{n}{12}\right)+\frac{1617629}{2103300} \Delta_{3,8}(n)-\frac{41411053}{1051650} \Delta_{3,8}\left(\frac{n}{2}\right)-\frac{963303712}{175275} \Delta_{3,8}\left(\frac{n}{4}\right) \\
& -\frac{501902144}{58425} \Delta_{3,8}\left(\frac{n}{8}\right)+\frac{10442}{23085} a_{4}(n)+\frac{42112}{513} a_{4}\left(\frac{n}{2}\right)+\frac{1886464}{405} a_{4}\left(\frac{n}{4}\right) \\
& -\frac{954}{19} a_{2}\left(\frac{n}{3}\right)+2 a_{3}(n)+\frac{1752}{19} a_{3}\left(\frac{n}{3}\right)-\frac{49868}{57} a_{5}(n)-\frac{2734720}{57} a_{5}\left(\frac{n}{2}\right)-14 a_{6}(n) \\
& -\frac{33312}{19} a_{6}\left(\frac{n}{2}\right)-\frac{44480}{57} a_{7}(n)-\frac{37568}{19} a_{8}(n)+\frac{58112}{19} a_{9}(n) \text {, } \\
& N\left(1,2,3^{9}, 6^{5} ; n\right)=\frac{1}{6691200} \sigma_{7}(n)-\frac{1}{6691200} \sigma_{7}\left(\frac{n}{2}\right)+\frac{1093}{2230400} \sigma_{7}\left(\frac{n}{3}\right)-\frac{1}{836400} \sigma_{7}\left(\frac{n}{4}\right) \\
& -\frac{1093}{2230400} \sigma_{7}\left(\frac{n}{6}\right)+\frac{16}{52275} \sigma_{7}\left(\frac{n}{8}\right)-\frac{1093}{278800} \sigma_{7}\left(\frac{n}{12}\right)+\frac{17488}{17425} \sigma_{7}\left(\frac{n}{24}\right)-\frac{7027}{22950} a_{1}(n) \\
& -\frac{125374}{1215} a_{1}\left(\frac{n}{2}\right)-\frac{37668}{425} a_{1}\left(\frac{n}{3}\right)+\frac{6899168}{11475} a_{1}\left(\frac{n}{4}\right)-\frac{8418}{5} a_{1}\left(\frac{n}{6}\right)-\frac{5834976}{425} a_{1}\left(\frac{n}{12}\right) \\
& +\frac{13423}{12300} \Delta_{3,8}(n)-\frac{1754749}{55350} \Delta_{3,8}\left(\frac{n}{2}\right)-\frac{233832964}{27675} \Delta_{3,8}\left(\frac{n}{4}\right)-\frac{34935616}{3075} \Delta_{3,8}\left(\frac{n}{8}\right) \\
& -\frac{106}{135} a_{4}(n)+\frac{27152}{243} a_{4}\left(\frac{n}{2}\right)+\frac{940288}{135} a_{4}\left(\frac{n}{4}\right)+2 a_{2}(n)+24 a_{2}\left(\frac{n}{3}\right) \\
& +30 a_{3}\left(\frac{n}{3}\right)-\frac{4576}{3} a_{5}(n)-71424 a_{5}\left(\frac{n}{2}\right)-12 a_{6}(n)-2656 a_{6}\left(\frac{n}{2}\right) \\
& -\frac{2912}{3} a_{7}(n)-3136 a_{8}(n)+4288 a_{9}(n) \text {, }
\end{aligned}
$$




$$
\begin{aligned}
& N\left(1,2,3^{11}, 6^{3} ; n\right)=\frac{1}{3345600} \sigma_{7}(n)-\frac{1}{3345600} \sigma_{7}\left(\frac{n}{2}\right)+\frac{1093}{1115200} \sigma_{7}\left(\frac{n}{3}\right)-\frac{1}{836400} \sigma_{7}\left(\frac{n}{4}\right) \\
& -\frac{1093}{1115200} \sigma_{7}\left(\frac{n}{6}\right)+\frac{16}{52275} \sigma_{7}\left(\frac{n}{8}\right)-\frac{1093}{278800} \sigma_{7}\left(\frac{n}{12}\right)+\frac{17488}{17425} \sigma_{7}\left(\frac{n}{24}\right)+\frac{2737708}{1962225} a_{1}(n) \\
& -\frac{3684128}{23085} a_{1}\left(\frac{n}{2}\right)-\frac{1119009}{8075} a_{1}\left(\frac{n}{3}\right)+\frac{576221536}{654075} a_{1}\left(\frac{n}{4}\right)-\frac{299196}{95} a_{1}\left(\frac{n}{6}\right) \\
& -\frac{298495584}{8075} a_{1}\left(\frac{n}{12}\right)+\frac{1793083}{1051650} \Delta_{3,8}(n)-\frac{2917237}{175275} \Delta_{3,8}\left(\frac{n}{2}\right)-\frac{7542242636}{525825} \Delta_{3,8}\left(\frac{n}{4}\right) \\
& -\frac{2978295232}{175275} \Delta_{3,8}\left(\frac{n}{8}\right)-\frac{71572}{23085} a_{4}(n)+\frac{785584}{4617} a_{4}\left(\frac{n}{2}\right)+\frac{4692224}{405} a_{4}\left(\frac{n}{4}\right)+6 a_{2}(n) \\
& \frac{3354}{19} a_{2}\left(\frac{n}{3}\right)-4 a_{3}(n)-\frac{2100}{19} a_{3}\left(\frac{n}{3}\right)-\frac{157936}{57} a_{5}(n)-\frac{6743488}{57} a_{5}\left(\frac{n}{2}\right)-8 a_{6}(n) \\
& -\frac{84832}{19} a_{6}\left(\frac{n}{2}\right)-\frac{26368}{19} a_{7}(n)-\frac{103680}{19} a_{8}(n)+\frac{129536}{19} a_{9}(n), \\
& N\left(1,2,3^{13}, 6 ; n\right)=\frac{1}{1672800} \sigma_{7}(n)-\frac{1}{1672800} \sigma_{7}\left(\frac{n}{2}\right)+\frac{1093}{557600} \sigma_{7}\left(\frac{n}{3}\right)-\frac{1}{836400} \sigma_{7}\left(\frac{n}{4}\right) \\
& -\frac{1093}{557600} \sigma_{7}\left(\frac{n}{6}\right)+\frac{16}{52275} \sigma_{7}\left(\frac{n}{8}\right)-\frac{1093}{278800} \sigma_{7}\left(\frac{n}{12}\right)+\frac{17488}{17425} \sigma_{7}\left(\frac{n}{24}\right)+\frac{9141551}{1962225} a_{1}(n) \\
& -\frac{1259116}{4617} a_{1}\left(\frac{n}{2}\right)-\frac{1906773}{8075} a_{1}\left(\frac{n}{3}\right)+\frac{953822816}{654075} a_{1}\left(\frac{n}{4}\right)-\frac{114768}{19} a_{1}\left(\frac{n}{6}\right) \\
& -\frac{685051104}{8075} a_{1}\left(\frac{n}{12}\right)+\frac{1526788}{525825} \Delta_{3,8}(n)+\frac{6615238}{525825} \Delta_{3,8}\left(\frac{n}{2}\right)-\frac{13736655476}{525825} \Delta_{3,8}\left(\frac{n}{4}\right) \\
& -\frac{5003340992}{175275} \Delta_{3,8}\left(\frac{n}{8}\right)-\frac{174584}{23085} a_{4}(n)+\frac{1318480}{4617} a_{4}\left(\frac{n}{2}\right)+\frac{8445184}{405} a_{4}\left(\frac{n}{4}\right) \\
& +14 a_{2}(n)+\frac{9114}{19} a_{2}\left(\frac{n}{3}\right)-12 a_{3}(n)-\frac{7632}{19} a_{3}\left(\frac{n}{3}\right)-\frac{99376}{19} a_{5}(n)-\frac{12086336}{57} a_{5}\left(\frac{n}{2}\right) \\
& -\frac{153632}{19} a_{6}\left(\frac{n}{2}\right)-\frac{129344}{57} a_{7}(n)-\frac{193152}{19} a_{8}(n)+\frac{228224}{19} a_{9}(n), \\
& N\left(1,2^{3}, 3,6^{11} ; n\right)=\frac{1}{21411840} \sigma_{7}(n)-\frac{1}{21411840} \sigma_{7}\left(\frac{n}{2}\right)-\frac{219}{7137280} \sigma_{7}\left(\frac{n}{3}\right)+\frac{1}{167280} \sigma_{7}\left(\frac{n}{4}\right) \\
& +\frac{219}{7137280} \sigma_{7}\left(\frac{n}{6}\right)-\frac{16}{10455} \sigma_{7}\left(\frac{n}{8}\right)-\frac{219}{55760} \sigma_{7}\left(\frac{n}{12}\right)+\frac{3504}{3485} \sigma_{7}\left(\frac{n}{24}\right)-\frac{76427711}{25116480} a_{1}(n) \\
& -\frac{773183}{18468} a_{1}\left(\frac{n}{2}\right)-\frac{1035447}{103360} a_{1}\left(\frac{n}{3}\right)+\frac{36065152}{130815} a_{1}\left(\frac{n}{4}\right)+\frac{10527}{76} a_{1}\left(\frac{n}{6}\right)+\frac{20941632}{1615} a_{1}\left(\frac{n}{12}\right) \\
& +\frac{416821}{3365280} \Delta_{3,8}(n)-\frac{28040579}{560880} \Delta_{3,8}\left(\frac{n}{2}\right)-\frac{131698787}{105165} \Delta_{3,8}\left(\frac{n}{4}\right)-\frac{166710464}{35055} \Delta_{3,8}\left(\frac{n}{8}\right) \\
& +\frac{53909}{18468} a_{4}(n)+\frac{178124}{4617} a_{4}\left(\frac{n}{2}\right)+\frac{111872}{81} a_{4}\left(\frac{n}{4}\right)-\frac{11}{4} a_{2}(n)-\frac{8991}{76} a_{2}\left(\frac{n}{3}\right) \\
& +\frac{19}{4} a_{3}(n)+\frac{7623}{76} a_{3}\left(\frac{n}{3}\right)+\frac{11645}{38} a_{5}(n)-\frac{275520}{19} a_{5}\left(\frac{n}{2}\right)-\frac{69}{4} a_{6}(n) \\
& -\frac{9088}{19} a_{6}\left(\frac{n}{2}\right)-\frac{36200}{57} a_{7}(n)-\frac{5440}{19} a_{8}(n)+\frac{29120}{19} a_{9}(n) \text {, }
\end{aligned}
$$




$$
\begin{aligned}
& N\left(1,2^{3}, 3^{3}, 6^{9} ; n\right)=\frac{1}{10705920} \sigma_{7}(n)-\frac{1}{10705920} \sigma_{7}\left(\frac{n}{2}\right)-\frac{219}{3568640} \sigma_{7}\left(\frac{n}{3}\right)+\frac{1}{167280} \sigma_{7}\left(\frac{n}{4}\right) \\
& +\frac{219}{3568640} \sigma_{7}\left(\frac{n}{6}\right)-\frac{16}{10455} \sigma_{7}\left(\frac{n}{8}\right)-\frac{219}{55760} \sigma_{7}\left(\frac{n}{12}\right)+\frac{3504}{3485} \sigma_{7}\left(\frac{n}{24}\right)-\frac{48947041}{12558240} a_{1}(n) \\
& -\frac{1530623}{46170} a_{1}\left(\frac{n}{2}\right)+\frac{647043}{51680} a_{1}\left(\frac{n}{3}\right)+\frac{29563808}{130815} a_{1}\left(\frac{n}{4}\right)+\frac{124899}{190} a_{1}\left(\frac{n}{6}\right)+\frac{28290528}{1615} a_{1}\left(\frac{n}{12}\right) \\
& -\frac{129737}{841320} \Delta_{3,8}(n)-\frac{1978996}{35055} \Delta_{3,8}\left(\frac{n}{2}\right)+\frac{18091228}{105165} \Delta_{3,8}\left(\frac{n}{4}\right)-\frac{129667456}{35055} \Delta_{3,8}\left(\frac{n}{8}\right) \\
& +\frac{18707}{4617} a_{4}(n)+\frac{105392}{4617} a_{4}\left(\frac{n}{2}\right)+\frac{27136}{81} a_{4}\left(\frac{n}{4}\right)-\frac{7}{2} a_{2}(n)-\frac{4581}{38} a_{2}\left(\frac{n}{3}\right) \\
& +\frac{11}{2} a_{3}(n)+\frac{2529}{38} a_{3}\left(\frac{n}{3}\right)+\frac{14477}{19} a_{5}(n)-\frac{70016}{19} a_{5}\left(\frac{n}{2}\right)-\frac{39}{2} a_{6}(n) \\
& -\frac{1184}{19} a_{6}\left(\frac{n}{2}\right)-\frac{37568}{57} a_{7}(n)+\frac{4288}{19} a_{8}(n)+\frac{23040}{19} a_{9}(n) \text {, } \\
& N\left(1,2^{3}, 3^{5}, 6^{7} ; n\right)=\frac{1}{5352960} \sigma_{7}(n)-\frac{1}{5352960} \sigma_{7}\left(\frac{n}{2}\right)-\frac{219}{1784320} \sigma_{7}\left(\frac{n}{3}\right)+\frac{1}{167280} \sigma_{7}\left(\frac{n}{4}\right) \\
& +\frac{219}{1784320} \sigma_{7}\left(\frac{n}{6}\right)-\frac{16}{10455} \sigma_{7}\left(\frac{n}{8}\right)-\frac{219}{55760} \sigma_{7}\left(\frac{n}{12}\right)+\frac{3504}{3485} \sigma_{7}\left(\frac{n}{24}\right)-\frac{709357}{139536} a_{1}(n) \\
& -\frac{116534}{7695} a_{1}\left(\frac{n}{2}\right)+\frac{223983}{5168} a_{1}\left(\frac{n}{3}\right)+\frac{3339424}{26163} a_{1}\left(\frac{n}{4}\right)+\frac{130086}{95} a_{1}\left(\frac{n}{6}\right)+\frac{8215776}{323} a_{1}\left(\frac{n}{12}\right) \\
& -\frac{1665}{3116} \Delta_{3,8}(n)-\frac{2382854}{35055} \Delta_{3,8}\left(\frac{n}{2}\right)+\frac{30845482}{11685} \Delta_{3,8}\left(\frac{n}{4}\right)-\frac{11609600}{7011} \Delta_{3,8}\left(\frac{n}{8}\right) \\
& +\frac{2882}{513} a_{4}(n)-\frac{824}{1539} a_{4}\left(\frac{n}{2}\right)-\frac{124928}{81} a_{4}\left(\frac{n}{4}\right)-5 a_{2}(n)-\frac{2871}{19} a_{2}\left(\frac{n}{3}\right)+7 a_{3}(n) \\
& +\frac{1161}{19} a_{3}\left(\frac{n}{3}\right)+\frac{27130}{19} a_{5}(n)+\frac{297728}{19} a_{5}\left(\frac{n}{2}\right)-23 a_{6}(n)+\frac{12832}{19} a_{6}\left(\frac{n}{2}\right) \\
& -\frac{11568}{19} a_{7}(n)+\frac{21952}{19} a_{8}(n)+\frac{8384}{19} a_{9}(n) \text {, } \\
& N\left(1,2^{3}, 3^{7}, 6^{5} ; n\right)=\frac{1}{2676480} \sigma_{7}(n)-\frac{1}{2676480} \sigma_{7}\left(\frac{n}{2}\right)-\frac{219}{892160} \sigma_{7}\left(\frac{n}{3}\right)+\frac{1}{167280} \sigma_{7}\left(\frac{n}{4}\right) \\
& +\frac{219}{892160} \sigma_{7}\left(\frac{n}{6}\right)-\frac{16}{10455} \sigma_{7}\left(\frac{n}{8}\right)-\frac{219}{55760} \sigma_{7}\left(\frac{n}{12}\right)+\frac{3504}{3485} \sigma_{7}\left(\frac{n}{24}\right)-\frac{21920611}{3139560} a_{1}(n) \\
& +\frac{102754}{4617} a_{1}\left(\frac{n}{2}\right)+\frac{1162653}{12920} a_{1}\left(\frac{n}{3}\right)-\frac{8790368}{130815} a_{1}\left(\frac{n}{4}\right)+\frac{48894}{19} a_{1}\left(\frac{n}{6}\right)+\frac{67527072}{1615} a_{1}\left(\frac{n}{12}\right) \\
& -\frac{466729}{420660} \Delta_{3,8}(n)-\frac{716801}{7790} \Delta_{3,8}\left(\frac{n}{2}\right)+\frac{758110888}{105165} \Delta_{3,8}\left(\frac{n}{4}\right)+\frac{82280896}{35055} \Delta_{3,8}\left(\frac{n}{8}\right) \\
& +\frac{37358}{4617} a_{4}(n)-\frac{177184}{4617} a_{4}\left(\frac{n}{2}\right)-\frac{411904}{81} a_{4}\left(\frac{n}{4}\right)-8 a_{2}(n)-\frac{4626}{19} a_{2}\left(\frac{n}{3}\right) \\
& +10 a_{3}(n)+\frac{2232}{19} a_{3}\left(\frac{n}{3}\right)+\frac{47244}{19} a_{5}(n)+\frac{987136}{19} a_{5}\left(\frac{n}{2}\right)-30 a_{6}(n) \\
& +\frac{39200}{19} a_{6}\left(\frac{n}{2}\right)-\frac{23168}{57} a_{7}(n)+\frac{55616}{19} a_{8}(n)-\frac{23680}{19} a_{9}(n) \text {, }
\end{aligned}
$$




$$
\begin{aligned}
& N\left(1,2^{3}, 3^{9}, 6^{3} ; n\right)=\frac{1}{1338240} \sigma_{7}(n)-\frac{1}{1338240} \sigma_{7}\left(\frac{n}{2}\right)-\frac{219}{446080} \sigma_{7}\left(\frac{n}{3}\right)+\frac{1}{167280} \sigma_{7}\left(\frac{n}{4}\right) \\
& +\frac{219}{446080} \sigma_{7}\left(\frac{n}{6}\right)-\frac{16}{10455} \sigma_{7}\left(\frac{n}{8}\right)-\frac{219}{55760} \sigma_{7}\left(\frac{n}{12}\right)+\frac{3504}{3485} \sigma_{7}\left(\frac{n}{24}\right)-\frac{8062919}{784890} a_{1}(n) \\
& +\frac{462686}{4617} a_{1}\left(\frac{n}{2}\right)+\frac{272571}{1615} a_{1}\left(\frac{n}{3}\right)-\frac{2259872}{4845} a_{1}\left(\frac{n}{4}\right)+\frac{92010}{19} a_{1}\left(\frac{n}{6}\right)+\frac{121990176}{1615} a_{1}\left(\frac{n}{12}\right) \\
& -\frac{875279}{420660} \Delta_{3,8}(n)-\frac{9835249}{70110} \Delta_{3,8}\left(\frac{n}{2}\right)+\frac{1690524524}{105165} \Delta_{3,8}\left(\frac{n}{4}\right)+\frac{122361536}{11685} \Delta_{3,8}\left(\frac{n}{8}\right) \\
& +\frac{57034}{4617} a_{4}(n)-\frac{494960}{4617} a_{4}\left(\frac{n}{2}\right)-12032 a_{4}\left(\frac{n}{4}\right)-14 a_{2}(n)-\frac{8712}{19} a_{2}\left(\frac{n}{3}\right)+16 a_{3}(n) \\
& +\frac{5634}{19} a_{3}\left(\frac{n}{3}\right)+\frac{82336}{19} a_{5}(n)+\frac{2332544}{19} a_{5}\left(\frac{n}{2}\right)-44 a_{6}(n)+\frac{90912}{19} a_{6}\left(\frac{n}{2}\right) \\
& +\frac{6752}{57} a_{7}(n)+\frac{121920}{19} a_{8}(n)-\frac{91840}{19} a_{9}(n), \\
& N\left(1,2^{3}, 3^{11}, 6 ; n\right)=\frac{1}{669120} \sigma_{7}(n)-\frac{1}{669120} \sigma_{7}\left(\frac{n}{2}\right)-\frac{219}{223040} \sigma_{7}\left(\frac{n}{3}\right)+\frac{1}{167280} \sigma_{7}\left(\frac{n}{4}\right) \\
& +\frac{219}{223040} \sigma_{7}\left(\frac{n}{6}\right)-\frac{16}{10455} \sigma_{7}\left(\frac{n}{8}\right)-\frac{219}{55760} \sigma_{7}\left(\frac{n}{12}\right)+\frac{3504}{3485} \sigma_{7}\left(\frac{n}{24}\right)-\frac{6337912}{392445} a_{1}(n) \\
& +\frac{6051688}{23085} a_{1}\left(\frac{n}{2}\right)+\frac{508371}{1615} a_{1}\left(\frac{n}{3}\right)-\frac{11386528}{8721} a_{1}\left(\frac{n}{4}\right)+\frac{888756}{95} a_{1}\left(\frac{n}{6}\right)+\frac{46913760}{323} a_{1}\left(\frac{n}{12}\right) \\
& -\frac{816157}{210330} \Delta_{3,8}(n)-\frac{8281841}{35055} \Delta_{3,8}\left(\frac{n}{2}\right)+\frac{3534320108}{105165} \Delta_{3,8}\left(\frac{n}{4}\right)+\frac{63950912}{2337} \Delta_{3,8}\left(\frac{n}{8}\right) \\
& +\frac{92476}{4617} a_{4}(n)-\frac{1099760}{4617} a_{4}\left(\frac{n}{2}\right)-\frac{699136}{27} a_{4}\left(\frac{n}{4}\right)-26 a_{2}(n)-\frac{17406}{19} a_{2}\left(\frac{n}{3}\right) \\
& +28 a_{3}(n)+\frac{13644}{19} a_{3}\left(\frac{n}{3}\right)+\frac{146032}{19} a_{5}(n)+\frac{5004864}{19} a_{5}\left(\frac{n}{2}\right)-72 a_{6}(n) \\
& +\frac{194016}{19} a_{6}\left(\frac{n}{2}\right)+\frac{78080}{57} a_{7}(n)+\frac{254208}{19} a_{8}(n)-\frac{236032}{19} a_{9}(n), \\
& N\left(1,2^{5}, 3,6^{9} ; n\right)=\frac{13}{107059200} \sigma_{7}(n)-\frac{13}{107059200} \sigma_{7}\left(\frac{n}{2}\right)+\frac{1089}{35686400} \sigma_{7}\left(\frac{n}{3}\right)-\frac{13}{836400} \sigma_{7}\left(\frac{n}{4}\right) \\
& -\frac{1089}{35686400} \sigma_{7}\left(\frac{n}{6}\right)+\frac{208}{52275} \sigma_{7}\left(\frac{n}{8}\right)-\frac{1089}{278800} \sigma_{7}\left(\frac{n}{12}\right)+\frac{17424}{17425} \sigma_{7}\left(\frac{n}{24}\right)-\frac{930617}{734400} a_{1}(n) \\
& -\frac{101227}{1620} a_{1}\left(\frac{n}{2}\right)-\frac{1474281}{27200} a_{1}\left(\frac{n}{3}\right)+\frac{13629632}{34425} a_{1}\left(\frac{n}{4}\right)-\frac{16047}{20} a_{1}\left(\frac{n}{6}\right)+\frac{2414592}{425} a_{1}\left(\frac{n}{12}\right) \\
& +\frac{65827}{98400} \Delta_{3,8}(n)-\frac{4069037}{147600} \Delta_{3,8}\left(\frac{n}{2}\right)-\frac{14171533}{3075} \Delta_{3,8}\left(\frac{n}{4}\right)-\frac{67518784}{9225} \Delta_{3,8}\left(\frac{n}{8}\right) \\
& +\frac{323}{540} a_{4}(n)+\frac{5420}{81} a_{4}\left(\frac{n}{2}\right)+\frac{1591552}{405} a_{4}\left(\frac{n}{4}\right)-\frac{3}{4} a_{2}(n)-\frac{405}{4} a_{2}\left(\frac{n}{3}\right)+\frac{11}{4} a_{3}(n) \\
& +\frac{621}{4} a_{3}\left(\frac{n}{3}\right)-\frac{1209}{2} a_{5}(n)-41280 a_{5}\left(\frac{n}{2}\right)-\frac{45}{4} a_{6}(n)-1472 a_{6}\left(\frac{n}{2}\right)-616 a_{7}(n) \\
& -1472 a_{8}(n)+2368 a_{9}(n) \text {, }
\end{aligned}
$$




$$
\begin{aligned}
& N\left(1,2^{5}, 3^{3}, 6^{7} ; n\right)=\sigma_{7}(n)-\frac{13}{53529600} \sigma_{7}\left(\frac{n}{2}\right)+\frac{1089}{17843200} \sigma_{7}\left(\frac{n}{3}\right) \\
& -\frac{13}{836400} \sigma_{7}\left(\frac{n}{4}\right)-\frac{1089}{17843200} \sigma_{7}\left(\frac{n}{6}\right)+\frac{208}{52275} \sigma_{7}\left(\frac{n}{8}\right) \\
& -\frac{1089}{278800} \sigma_{7}\left(\frac{n}{12}\right)+\frac{17424}{17425} \sigma_{7}\left(\frac{n}{24}\right)+\frac{14660221}{20930400} a_{1}(n) \\
& -\frac{1110329}{15390} a_{1}\left(\frac{n}{2}\right)-\frac{26966349}{258400} a_{1}\left(\frac{n}{3}\right)+\frac{33471712}{72675} a_{1}\left(\frac{n}{4}\right) \\
& -\frac{356409}{190} a_{1}\left(\frac{n}{6}\right)-\frac{7856352}{8075} a_{1}\left(\frac{n}{12}\right)+\frac{903031}{701100} \Delta_{3,8}(n) \\
& -\frac{2784353}{233700} \Delta_{3,8}\left(\frac{n}{2}\right)-\frac{1240047556}{175275} \Delta_{3,8}\left(\frac{n}{4}\right)-\frac{513576832}{58425} \Delta_{3,8}\left(\frac{n}{8}\right) \\
& -\frac{15302}{7695} a_{4}(n)+\frac{144368}{1539} a_{4}\left(\frac{n}{2}\right)+\frac{255488}{45} a_{4}\left(\frac{n}{4}\right)+\frac{1}{2} a_{2}(n) \\
& -\frac{5265}{38} a_{2}\left(\frac{n}{3}\right)+\frac{3}{2} a_{3}(n)+\frac{11421}{38} a_{3}\left(\frac{n}{3}\right)-\frac{29451}{19} a_{5}(n) \\
& -\frac{1132800}{19} a_{5}\left(\frac{n}{2}\right)-\frac{11}{2} a_{6}(n)-\frac{41312}{19} a_{6}\left(\frac{n}{2}\right) \\
& -\frac{8064}{19} a_{7}(n)-\frac{44352}{19} a_{8}(n)+\frac{49792}{19} a_{9}(n) \text {, } \\
& N\left(1,2^{5}, 3^{5}, 6^{5} ; n\right)=\frac{13}{26764800} \sigma_{7}(n)-\frac{13}{26764800} \sigma_{7}\left(\frac{n}{2}\right) \\
& +\frac{1089}{8921600} \sigma_{7}\left(\frac{n}{3}\right)-\frac{13}{836400} \sigma_{7}\left(\frac{n}{4}\right)-\frac{1089}{8921600} \sigma_{7}\left(\frac{n}{6}\right) \\
& +\frac{208}{52275} \sigma_{7}\left(\frac{n}{8}\right)-\frac{1089}{278800} \sigma_{7}\left(\frac{n}{12}\right)+\frac{17424}{17425} \sigma_{7}\left(\frac{n}{24}\right) \\
& +\frac{3886589}{1162800} a_{1}(n)-\frac{743798}{7695} a_{1}\left(\frac{n}{2}\right)-\frac{21525669}{129200} a_{1}\left(\frac{n}{3}\right) \\
& +\frac{398828128}{654075} a_{1}\left(\frac{n}{4}\right)-\frac{305658}{95} a_{1}\left(\frac{n}{6}\right)-\frac{106168032}{8075} a_{1}\left(\frac{n}{12}\right) \\
& +\frac{120131}{58425} \Delta_{3,8}(n)+\frac{7642681}{350550} \Delta_{3,8}\left(\frac{n}{2}\right)-\frac{661236142}{58425} \Delta_{3,8}\left(\frac{n}{4}\right) \\
& -\frac{2099275136}{175275} \Delta_{3,8}\left(\frac{n}{8}\right)-\frac{4616}{855} a_{4}(n)+\frac{192712}{1539} a_{4}\left(\frac{n}{2}\right) \\
& +\frac{3594752}{405} a_{4}\left(\frac{n}{4}\right)+3 a_{2}(n)-\frac{2295}{19} a_{2}\left(\frac{n}{3}\right)-a_{3}(n)+\frac{7425}{19} a_{3}\left(\frac{n}{3}\right) \\
& -\frac{52854}{19} a_{5}(n)-\frac{1765248}{19} a_{5}\left(\frac{n}{2}\right)+5 a_{6}(n)-\frac{65184}{19} a_{6}\left(\frac{n}{2}\right) \\
& -\frac{6960}{19} a_{7}(n)-\frac{74304}{19} a_{8}(n)+\frac{69568}{19} a_{9}(n) \text {, }
\end{aligned}
$$




$$
\begin{aligned}
& N\left(1,2^{5}, 3^{7}, 6^{3} ; n\right)=\frac{13}{13382400} \sigma_{7}(n)-\frac{13}{13382400} \sigma_{7}\left(\frac{n}{2}\right)+\frac{1089}{4460800} \sigma_{7}\left(\frac{n}{3}\right)-\frac{13}{836400} \sigma_{7}\left(\frac{n}{4}\right) \\
& -\frac{1089}{4460800} \sigma_{7}\left(\frac{n}{6}\right)+\frac{208}{52275} \sigma_{7}\left(\frac{n}{8}\right)-\frac{1089}{278800} \sigma_{7}\left(\frac{n}{12}\right)+\frac{17424}{17425} \sigma_{7}\left(\frac{n}{24}\right)+\frac{38969711}{5232600} a_{1}(n) \\
& -\frac{1151386}{7695} a_{1}\left(\frac{n}{2}\right)-\frac{16236459}{64600} a_{1}\left(\frac{n}{3}\right)+\frac{594178528}{654075} a_{1}\left(\frac{n}{4}\right)-\frac{505386}{95} a_{1}\left(\frac{n}{6}\right)-\frac{315472032}{8075} a_{1}\left(\frac{n}{12}\right) \\
& +\frac{2174459}{701100} \Delta_{3,8}(n)+\frac{32259157}{350550} \Delta_{3,8}\left(\frac{n}{2}\right)-\frac{3315338816}{175275} \Delta_{3,8}\left(\frac{n}{4}\right)-\frac{3194860736}{175275} \Delta_{3,8}\left(\frac{n}{8}\right) \\
& -\frac{81178}{7695} a_{4}(n)+\frac{255488}{1539} a_{4}\left(\frac{n}{2}\right)+\frac{5979392}{405} a_{4}\left(\frac{n}{4}\right)+8 a_{2}(n)+\frac{270}{19} a_{2}\left(\frac{n}{3}\right)-6 a_{3}(n) \\
& +\frac{6912}{19} a_{3}\left(\frac{n}{3}\right)-\frac{86484}{19} a_{5}(n)-\frac{2918016}{19} a_{5}\left(\frac{n}{2}\right)+26 a_{6}(n)-\frac{108960}{19} a_{6}\left(\frac{n}{2}\right) \\
& -\frac{10304}{19} a_{7}(n)-\frac{130240}{19} a_{8}(n)+\frac{116992}{19} a_{9}(n) \text {, } \\
& N\left(1,2^{5}, 3^{9}, 6 ; n\right)=\sigma_{7}(n)-\frac{13}{6691200} \sigma_{7}\left(\frac{n}{2}\right)+\frac{1089}{2230400} \sigma_{7}\left(\frac{n}{3}\right)-\frac{13}{836400} \sigma_{7}\left(\frac{n}{4}\right) \\
& -\frac{1089}{2230400} \sigma_{7}\left(\frac{n}{6}\right)+\frac{208}{52275} \sigma_{7}\left(\frac{n}{8}\right)-\frac{1089}{278800} \sigma_{7}\left(\frac{n}{12}\right)+\frac{17424}{17425} \sigma_{7}\left(\frac{n}{24}\right)+\frac{18671533}{1308150} a_{1}(n) \\
& -\frac{2072086}{7695} a_{1}\left(\frac{n}{2}\right)-\frac{3101526}{8075} a_{1}\left(\frac{n}{3}\right)+\frac{337131296}{218025} a_{1}\left(\frac{n}{4}\right)-\frac{885726}{95} a_{1}\left(\frac{n}{6}\right)-\frac{756797472}{8075} a_{1}\left(\frac{n}{12}\right) \\
& +\frac{3322483}{701100} \Delta_{3,8}(n)+\frac{27336373}{116850} \Delta_{3,8}\left(\frac{n}{2}\right)-\frac{5896404436}{175275} \Delta_{3,8}\left(\frac{n}{4}\right)-\frac{1830837952}{58425} \Delta_{3,8}\left(\frac{n}{8}\right) \\
& -\frac{146306}{7695} a_{4}(n)+\frac{358736}{1539} a_{4}\left(\frac{n}{2}\right)+\frac{3560704}{135} a_{4}\left(\frac{n}{4}\right)+18 a_{2}(n)+\frac{7344}{19} a_{2}\left(\frac{n}{3}\right)-16 a_{3}(n) \\
& +\frac{1890}{19} a_{3}\left(\frac{n}{3}\right)-\frac{139488}{19} a_{5}(n)-\frac{5175552}{19} a_{5}\left(\frac{n}{2}\right)+68 a_{6}(n)-\frac{195488}{19} a_{6}\left(\frac{n}{2}\right) \\
& -\frac{24544}{19} a_{7}(n)-\frac{241088}{19} a_{8}(n)+\frac{225600}{19} a_{9}(n) \text {, } \\
& N\left(1,2^{7}, 3,6^{7} ; n\right)=\frac{1}{2611200} \sigma_{7}(n)-\frac{1}{2611200} \sigma_{7}\left(\frac{n}{2}\right)-\frac{27}{870400} \sigma_{7}\left(\frac{n}{3}\right)+\frac{1}{20400} \sigma_{7}\left(\frac{n}{4}\right) \\
& +\frac{27}{870400} \sigma_{7}\left(\frac{n}{6}\right)-\frac{16}{1275} \sigma_{7}\left(\frac{n}{8}\right)-\frac{27}{6800} \sigma_{7}\left(\frac{n}{12}\right)+\frac{432}{425} \sigma_{7}\left(\frac{n}{24}\right)-\frac{26650837}{4651200} a_{1}(n) \\
& -\frac{422639}{10260} a_{1}\left(\frac{n}{2}\right)+\frac{28740177}{516800} a_{1}\left(\frac{n}{3}\right)+\frac{47870848}{218025} a_{1}\left(\frac{n}{4}\right)+\frac{347463}{380} a_{1}\left(\frac{n}{6}\right)+\frac{136102464}{8075} a_{1}\left(\frac{n}{12}\right) \\
& -\frac{10433}{15200} \Delta_{3,8}(n)-\frac{1726417}{22800} \Delta_{3,8}\left(\frac{n}{2}\right)+\frac{565303}{475} \Delta_{3,8}\left(\frac{n}{4}\right)-\frac{4867136}{1425} \Delta_{3,8}\left(\frac{n}{8}\right) \\
& +\frac{21943}{3420} a_{4}(n)+\frac{12316}{513} a_{4}\left(\frac{n}{2}\right)-\frac{49408}{135} a_{4}\left(\frac{n}{4}\right)-\frac{15}{4} a_{2}(n)-\frac{4131}{76} a_{2}\left(\frac{n}{3}\right) \\
& +\frac{23}{4} a_{3}(n)-\frac{8181}{76} a_{3}\left(\frac{n}{3}\right)+\frac{56673}{38} a_{5}(n)+\frac{80064}{19} a_{5}\left(\frac{n}{2}\right)-\frac{105}{4} a_{6}(n) \\
& +\frac{3712}{19} a_{6}\left(\frac{n}{2}\right)-\frac{17496}{19} a_{7}(n)+\frac{12224}{19} a_{8}(n)+\frac{27840}{19} a_{9}(n) \text {, }
\end{aligned}
$$




$$
\begin{aligned}
& N\left(1,2^{7}, 3^{3}, 6^{5} ; n\right)=\frac{1}{1305600} \sigma_{7}(n)-\frac{1}{1305600} \sigma_{7}\left(\frac{n}{2}\right)-\frac{27}{435200} \sigma_{7}\left(\frac{n}{3}\right)+\frac{1}{20400} \sigma_{7}\left(\frac{n}{4}\right) \\
& +\frac{27}{435200} \sigma_{7}\left(\frac{n}{6}\right)-\frac{16}{1275} \sigma_{7}\left(\frac{n}{8}\right)-\frac{27}{6800} \sigma_{7}\left(\frac{n}{12}\right)+\frac{432}{425} \sigma_{7}\left(\frac{n}{24}\right)-\frac{226286603}{20930400} a_{1}(n) \\
& -\frac{12797}{342} a_{1}\left(\frac{n}{2}\right)+\frac{49457907}{258400} a_{1}\left(\frac{n}{3}\right)+\frac{85331104}{654075} a_{1}\left(\frac{n}{4}\right)+\frac{129699}{38} a_{1}\left(\frac{n}{6}\right)+\frac{191353824}{8075} a_{1}\left(\frac{n}{12}\right) \\
& -\frac{80801}{34200} \Delta_{3,8}(n)-\frac{875719}{8550} \Delta_{3,8}\left(\frac{n}{2}\right)+\frac{26064172}{4275} \Delta_{3,8}\left(\frac{n}{4}\right)-\frac{5024128}{4275} \Delta_{3,8}\left(\frac{n}{8}\right) \\
& +\frac{101371}{7695} a_{4}(n)-\frac{7568}{171} a_{4}\left(\frac{n}{2}\right)-\frac{1467904}{405} a_{4}\left(\frac{n}{4}\right)-\frac{11}{2} a_{2}(n)+\frac{5319}{38} a_{2}\left(\frac{n}{3}\right) \\
& +\frac{15}{2} a_{3}(n)-\frac{23787}{38} a_{3}\left(\frac{n}{3}\right)+\frac{75249}{19} a_{5}(n)+\frac{746880}{19} a_{5}\left(\frac{n}{2}\right)-\frac{75}{2} a_{6}(n) \\
& +\frac{28896}{19} a_{6}\left(\frac{n}{2}\right)-\frac{32000}{19} a_{7}(n)+\frac{41664}{19} a_{8}(n)+\frac{35840}{19} a_{9}(n) \text {, } \\
& N\left(1,2^{7}, 3^{5}, 6^{3} ; n\right)=\frac{1}{652800} \sigma_{7}(n)-\frac{1}{652800} \sigma_{7}\left(\frac{n}{2}\right)-\frac{27}{217600} \sigma_{7}\left(\frac{n}{3}\right)+\frac{1}{20400} \sigma_{7}\left(\frac{n}{4}\right) \\
& +\frac{27}{217600} \sigma_{7}\left(\frac{n}{6}\right)-\frac{16}{1275} \sigma_{7}\left(\frac{n}{8}\right)-\frac{27}{6800} \sigma_{7}\left(\frac{n}{12}\right)+\frac{432}{425} \sigma_{7}\left(\frac{n}{24}\right)-\frac{178254803}{10465200} a_{1}(n) \\
& -\frac{13798}{855} a_{1}\left(\frac{n}{2}\right)+\frac{44041707}{129200} a_{1}\left(\frac{n}{3}\right)-\frac{42759136}{654075} a_{1}\left(\frac{n}{4}\right)+\frac{576918}{95} a_{1}\left(\frac{n}{6}\right)+\frac{301317984}{8075} a_{1}\left(\frac{n}{12}\right) \\
& -\frac{71951}{17100} \Delta_{3,8}(n)-\frac{729679}{4275} \Delta_{3,8}\left(\frac{n}{2}\right)+\frac{57412142}{4275} \Delta_{3,8}\left(\frac{n}{4}\right)+\frac{14052352}{4275} \Delta_{3,8}\left(\frac{n}{8}\right) \\
& +\frac{163442}{7695} a_{4}(n)-\frac{17560}{171} a_{4}\left(\frac{n}{2}\right)-\frac{3620864}{405} a_{4}\left(\frac{n}{4}\right)-9 a_{2}(n)+\frac{4509}{19} a_{2}\left(\frac{n}{3}\right) \\
& +11 a_{3}(n)-\frac{19899}{19} a_{3}\left(\frac{n}{3}\right)+\frac{127458}{19} a_{5}(n)+\frac{1820928}{19} a_{5}\left(\frac{n}{2}\right)-59 a_{6}(n) \\
& +\frac{68512}{19} a_{6}\left(\frac{n}{2}\right)-\frac{43120}{19} a_{7}(n)+\frac{89792}{19} a_{8}(n)+\frac{23488}{19} a_{9}(n) \text {, } \\
& N\left(1,2^{7}, 3^{7}, 6 ; n\right)=\frac{1}{326400} \sigma_{7}(n)-\frac{1}{326400} \sigma_{7}\left(\frac{n}{2}\right)-\frac{27}{108800} \sigma_{7}\left(\frac{n}{3}\right)+\frac{1}{20400} \sigma_{7}\left(\frac{n}{4}\right) \\
& +\frac{27}{108800} \sigma_{7}\left(\frac{n}{6}\right)-\frac{16}{1275} \sigma_{7}\left(\frac{n}{8}\right)-\frac{27}{6800} \sigma_{7}\left(\frac{n}{12}\right)+\frac{432}{425} \sigma_{7}\left(\frac{n}{24}\right)-\frac{136362553}{5232600} a_{1}(n) \\
& +\frac{14210}{513} a_{1}\left(\frac{n}{2}\right)+\frac{32941557}{64600} a_{1}\left(\frac{n}{3}\right)-\frac{231124576}{654075} a_{1}\left(\frac{n}{4}\right)+\frac{178974}{19} a_{1}\left(\frac{n}{6}\right)+\frac{537354144}{8075} a_{1}\left(\frac{n}{12}\right) \\
& -\frac{107627}{17100} \Delta_{3,8}(n)-\frac{2692001}{8550} \Delta_{3,8}\left(\frac{n}{2}\right)+\frac{104633432}{4275} \Delta_{3,8}\left(\frac{n}{4}\right)+\frac{42576832}{4275} \Delta_{3,8}\left(\frac{n}{8}\right) \\
& +\frac{248954}{7695} a_{4}(n)-\frac{74528}{513} a_{4}\left(\frac{n}{2}\right)-\frac{6965504}{405} a_{4}\left(\frac{n}{4}\right)-16 a_{2}(n)+\frac{2646}{19} a_{2}\left(\frac{n}{3}\right) \\
& +18 a_{3}(n)-\frac{24192}{19} a_{3}\left(\frac{n}{3}\right)+\frac{191772}{19} a_{5}(n)+\frac{3475200}{19} a_{5}\left(\frac{n}{2}\right)-102 a_{6}(n) \\
& +\frac{129952}{19} a_{6}\left(\frac{n}{2}\right)-\frac{52096}{19} a_{7}(n)+\frac{168256}{19} a_{8}(n)-\frac{15488}{19} a_{9}(n) \text {, }
\end{aligned}
$$




$$
\begin{aligned}
& N\left(1,2^{9}, 3,6^{5} ; n\right)=\frac{121}{107059200} \sigma_{7}(n)-\frac{121}{107059200} \sigma_{7}\left(\frac{n}{2}\right)+\frac{1053}{35686400} \sigma_{7}\left(\frac{n}{3}\right) \\
& -\frac{121}{836400} \sigma_{7}\left(\frac{n}{4}\right)-\frac{1053}{35686400} \sigma_{7}\left(\frac{n}{6}\right)+\frac{1936}{52275} \sigma_{7}\left(\frac{n}{8}\right)-\frac{1053}{278800} \sigma_{7}\left(\frac{n}{12}\right)+\frac{16848}{17425} \sigma_{7}\left(\frac{n}{24}\right) \\
& +\frac{2420547}{516800} a_{1}(n)-\frac{101747}{2052} a_{1}\left(\frac{n}{2}\right)-\frac{110128383}{516800} a_{1}\left(\frac{n}{3}\right)+\frac{93447872}{218025} a_{1}\left(\frac{n}{4}\right)-\frac{254853}{76} a_{1}\left(\frac{n}{6}\right) \\
& +\frac{63127296}{8075} a_{1}\left(\frac{n}{12}\right)+\frac{4918261}{1869600} \Delta_{3,8}(n)+\frac{10977943}{934800} \Delta_{3,8}\left(\frac{n}{2}\right)-\frac{549806939}{58425} \Delta_{3,8}\left(\frac{n}{4}\right) \\
& -\frac{504268864}{58425} \Delta_{3,8}\left(\frac{n}{8}\right)-\frac{8339}{1140} a_{4}(n)+\frac{68540}{513} a_{4}\left(\frac{n}{2}\right)+\frac{947968}{135} a_{4}\left(\frac{n}{4}\right)+\frac{1}{4} a_{2}(n) \\
& -\frac{30699}{76} a_{2}\left(\frac{n}{3}\right)+\frac{7}{4} a_{3}(n)+\frac{67635}{76} a_{3}\left(\frac{n}{3}\right)-\frac{126927}{38} a_{5}(n)-\frac{1444032}{19} a_{5}\left(\frac{n}{2}\right) \\
& +\frac{7}{4} a_{6}(n)-\frac{51264}{19} a_{6}\left(\frac{n}{2}\right)+\frac{10536}{19} a_{7}(n)-\frac{51264}{19} a_{8}(n)+\frac{18624}{19} a_{9}(n), \\
& N\left(1,2^{9}, 3^{3}, 6^{3} ; n\right)=\frac{121}{53529600} \sigma_{7}(n)-\frac{121}{53529600} \sigma_{7}\left(\frac{n}{2}\right)+\frac{1053}{17843200} \sigma_{7}\left(\frac{n}{3}\right)-\frac{121}{836400} \sigma_{7}\left(\frac{n}{4}\right) \\
& -\frac{1053}{17843200} \sigma_{7}\left(\frac{n}{6}\right)+\frac{1936}{52275} \sigma_{7}\left(\frac{n}{8}\right)-\frac{1053}{278800} \sigma_{7}\left(\frac{n}{12}\right)+\frac{16848}{17425} \sigma_{7}\left(\frac{n}{24}\right)+\frac{374620577}{20930400} a_{1}(n) \\
& -\frac{124711}{5130} a_{1}\left(\frac{n}{2}\right)-\frac{152510913}{258400} a_{1}\left(\frac{n}{3}\right)+\frac{323594336}{654075} a_{1}\left(\frac{n}{4}\right)-\frac{1828773}{190} a_{1}\left(\frac{n}{6}\right)+\frac{65306016}{8075} a_{1}\left(\frac{n}{12}\right) \\
& +\frac{1277093}{175275} \Delta_{3,8}(n)+\frac{31311067}{701100} \Delta_{3,8}\left(\frac{n}{2}\right)-\frac{3480236252}{175275} \Delta_{3,8}\left(\frac{n}{4}\right)-\frac{1978133632}{175275} \Delta_{3,8}\left(\frac{n}{8}\right) \\
& -\frac{193804}{7695} a_{4}(n)+\frac{165616}{513} a_{4}\left(\frac{n}{2}\right)+\frac{5430784}{405} a_{4}\left(\frac{n}{4}\right)+\frac{5}{2} a_{2}(n)-\frac{43389}{38} a_{2}\left(\frac{n}{3}\right) \\
& -\frac{1}{2} a_{3}(n)+\frac{98793}{38} a_{3}\left(\frac{n}{3}\right)-\frac{191583}{19} a_{5}(n)-\frac{2798592}{19} a_{5}\left(\frac{n}{2}\right)+\frac{41}{2} a_{6}(n) \\
& -\frac{101344}{19} a_{6}\left(\frac{n}{2}\right)+\frac{59840}{19} a_{7}(n)-\frac{106816}{19} a_{8}(n)-\frac{39296}{19} a_{9}(n) \text {, } \\
& N\left(1,2^{9}, 3^{5}, 6 ; n\right)=\frac{121}{26764800} \sigma_{7}(n)-\frac{121}{26764800} \sigma_{7}\left(\frac{n}{2}\right)+\frac{1053}{8921600} \sigma_{7}\left(\frac{n}{3}\right)-\frac{121}{836400} \sigma_{7}\left(\frac{n}{4}\right) \\
& -\frac{1053}{8921600} \sigma_{7}\left(\frac{n}{6}\right)+\frac{1936}{52275} \sigma_{7}\left(\frac{n}{8}\right)-\frac{1053}{278800} \sigma_{7}\left(\frac{n}{12}\right)+\frac{16848}{17425} \sigma_{7}\left(\frac{n}{24}\right)+\frac{348118937}{10465200} a_{1}(n) \\
& +\frac{9934}{2565} a_{1}\left(\frac{n}{2}\right)-\frac{126054153}{129200} a_{1}\left(\frac{n}{3}\right)+\frac{361337056}{654075} a_{1}\left(\frac{n}{4}\right)-\frac{1412298}{95} a_{1}\left(\frac{n}{6}\right)+\frac{44351136}{8075} a_{1}\left(\frac{n}{12}\right) \\
& +\frac{4222157}{350550} \Delta_{3,8}(n)+\frac{55114027}{350550} \Delta_{3,8}\left(\frac{n}{2}\right)-\frac{5650411762}{175275} \Delta_{3,8}\left(\frac{n}{4}\right)-\frac{2498367872}{175275} \Delta_{3,8}\left(\frac{n}{8}\right) \\
& -\frac{348668}{7695} a_{4}(n)+\frac{235768}{513} a_{4}\left(\frac{n}{2}\right)+\frac{8743424}{405} a_{4}\left(\frac{n}{4}\right)+7 a_{2}(n)-\frac{32859}{19} a_{2}\left(\frac{n}{3}\right) \\
& -5 a_{3}(n)+\frac{79029}{19} a_{3}\left(\frac{n}{3}\right)-\frac{324414}{19} a_{5}(n)-\frac{4531584}{19} a_{5}\left(\frac{n}{2}\right)+57 a_{6}(n) \\
& -\frac{162080}{19} a_{6}\left(\frac{n}{2}\right)+\frac{109072}{19} a_{7}(n)-\frac{178496}{19} a_{8}(n)-\frac{86848}{19} a_{9}(n) \text {, }
\end{aligned}
$$




$$
\begin{aligned}
& N\left(1,2^{11}, 3,6^{3} ; n\right)=\frac{73}{21411840} \sigma_{7}(n)-\frac{73}{21411840} \sigma_{7}\left(\frac{n}{2}\right)-\frac{243}{7137280} \sigma_{7}\left(\frac{n}{3}\right)+\frac{73}{167280} \sigma_{7}\left(\frac{n}{4}\right) \\
& +\frac{243}{7137280} \sigma_{7}\left(\frac{n}{6}\right)-\frac{1168}{10455} \sigma_{7}\left(\frac{n}{8}\right)-\frac{243}{55760} \sigma_{7}\left(\frac{n}{12}\right)+\frac{3888}{3485} \sigma_{7}\left(\frac{n}{24}\right)-\frac{189044797}{8372160} a_{1}(n) \\
& -\frac{377993}{3420} a_{1}\left(\frac{n}{2}\right)+\frac{56204433}{103360} a_{1}\left(\frac{n}{3}\right)+\frac{8630144}{26163} a_{1}\left(\frac{n}{4}\right)+\frac{3051603}{380} a_{1}\left(\frac{n}{6}\right)+\frac{2018880}{323} a_{1}\left(\frac{n}{12}\right) \\
& -\frac{7530433}{1121760} \Delta_{3,8}(n)-\frac{53148667}{560880} \Delta_{3,8}\left(\frac{n}{2}\right)+\frac{414610967}{35055} \Delta_{3,8}\left(\frac{n}{4}\right)-\frac{25619392}{7011} \Delta_{3,8}\left(\frac{n}{8}\right) \\
& +\frac{180319}{6156} a_{4}(n)+-\frac{34748}{171} a_{4}\left(\frac{n}{2}\right)-\frac{508672}{81} a_{4}\left(\frac{n}{4}\right)-\frac{19}{4} a_{2}(n)+\frac{76761}{76} a_{2}\left(\frac{n}{3}\right) \\
& +\frac{27}{4} a_{3}(n)-\frac{187569}{76} a_{3}\left(\frac{n}{3}\right)+\frac{384741}{38} a_{5}(n)+\frac{1384896}{19} a_{5}\left(\frac{n}{2}\right)-\frac{173}{4} a_{6}(n) \\
& +\frac{50304}{19} a_{6}\left(\frac{n}{2}\right)-\frac{88120}{19} a_{7}(n)+\frac{63680}{19} a_{8}(n)+\frac{134080}{19} a_{9}(n), \\
& N\left(1,2^{11}, 3^{3}, 6 ; n\right)=\frac{73}{10705920} \sigma_{7}(n)-\frac{73}{10705920} \sigma_{7}\left(\frac{n}{2}\right)-\frac{243}{3568640} \sigma_{7}\left(\frac{n}{3}\right)+\frac{73}{167280} \sigma_{7}\left(\frac{n}{4}\right) \\
& +\frac{243}{3568640} \sigma_{7}\left(\frac{n}{6}\right)-\frac{1168}{10455} \sigma_{7}\left(\frac{n}{8}\right)-\frac{243}{55760} \sigma_{7}\left(\frac{n}{12}\right)+\frac{3888}{3485} \sigma_{7}\left(\frac{n}{24}\right)-\frac{245957363}{4186080} a_{1}(n) \\
& -\frac{23035}{114} a_{1}\left(\frac{n}{2}\right)+\frac{85846347}{51680} a_{1}\left(\frac{n}{3}\right)+\frac{21952672}{130815} a_{1}\left(\frac{n}{4}\right)+\frac{960831}{38} a_{1}\left(\frac{n}{6}\right)-\frac{28109088}{1615} a_{1}\left(\frac{n}{12}\right) \\
& -\frac{5751031}{280440} \Delta_{3,8}(n)-\frac{3662827}{35055} \Delta_{3,8}\left(\frac{n}{2}\right)+\frac{1403022116}{35055} \Delta_{3,8}\left(\frac{n}{4}\right)+\frac{110448256}{35055} \Delta_{3,8}\left(\frac{n}{8}\right) \\
& +\frac{121981}{1539} a_{4}(n)-\frac{15152}{19} a_{4}\left(\frac{n}{2}\right)-\frac{1873408}{81} a_{4}\left(\frac{n}{4}\right)-\frac{15}{2} a_{2}(n)+\frac{131571}{38} a_{2}\left(\frac{n}{3}\right) \\
& +\frac{19}{2} a_{3}(n)-\frac{297783}{38} a_{3}\left(\frac{n}{3}\right)+\frac{566901}{19} a_{5}(n)+\frac{5008512}{19} a_{5}\left(\frac{n}{2}\right)-\frac{143}{2} a_{6}(n) \\
& +\frac{181856}{19} a_{6}\left(\frac{n}{2}\right)-\frac{236224}{19} a_{7}(n)+\frac{201920}{19} a_{8}(n)+\frac{328192}{19} a_{9}(n), \\
& N\left(1,2^{13}, 3,6 ; n\right)=\frac{1093}{107059200} \sigma_{7}(n)-\frac{1093}{107059200} \sigma_{7}\left(\frac{n}{2}\right)+\frac{729}{35686400} \sigma_{7}\left(\frac{n}{3}\right)-\frac{1093}{836400} \sigma_{7}\left(\frac{n}{4}\right) \\
& -\frac{729}{35686400} \sigma_{7}\left(\frac{n}{6}\right)+\frac{17488}{52275} \sigma_{7}\left(\frac{n}{8}\right)-\frac{729}{278800} \sigma_{7}\left(\frac{n}{12}\right)+\frac{11664}{17425} \sigma_{7}\left(\frac{n}{24}\right)+\frac{724141757}{13953600} a_{1}(n) \\
& +\frac{1279069}{10260} a_{1}\left(\frac{n}{2}\right)-\frac{861519699}{516800} a_{1}\left(\frac{n}{3}\right)+\frac{64369856}{218025} a_{1}\left(\frac{n}{4}\right)-\frac{10361493}{380} a_{1}\left(\frac{n}{6}\right)+\frac{302980608}{8075} a_{1}\left(\frac{n}{12}\right) \\
& +\frac{38477233}{1869600} \Delta_{3,8}(n)-\frac{66763541}{934800} \Delta_{3,8}\left(\frac{n}{2}\right)-\frac{2394970367}{58425} \Delta_{3,8}\left(\frac{n}{4}\right)-\frac{634891072}{58425} \Delta_{3,8}\left(\frac{n}{8}\right) \\
& -\frac{743663}{10260} a_{4}(n)+\frac{496396}{513} a_{4}\left(\frac{n}{2}\right)+\frac{3230464}{135} a_{4}\left(\frac{n}{4}\right)+\frac{5}{4} a_{2}(n)-\frac{292167}{76} a_{2}\left(\frac{n}{3}\right) \\
& +\frac{3}{4} a_{3}(n)+\frac{624591}{76} a_{3}\left(\frac{n}{3}\right)-\frac{1114371}{38} a_{5}(n)-\frac{5141952}{19} a_{5}\left(\frac{n}{2}\right)+\frac{91}{4} a_{6}(n) \\
& -\frac{190272}{19} a_{6}\left(\frac{n}{2}\right)+\frac{222216}{19} a_{7}(n)-\frac{190272}{19} a_{8}(n)+-\frac{301632}{19} a_{9}(n),
\end{aligned}
$$




$$
\begin{aligned}
& N\left(1^{2}, 6^{14} ; n\right)=\frac{1}{53529600} \sigma_{7}(n)-\frac{1}{53529600} \sigma_{7}\left(\frac{n}{2}\right)-\frac{547}{17843200} \sigma_{7}\left(\frac{n}{3}\right)+\frac{1}{418200} \sigma_{7}\left(\frac{n}{4}\right) \\
& +\frac{547}{17843200} \sigma_{7}\left(\frac{n}{6}\right)-\frac{32}{52275} \sigma_{7}\left(\frac{n}{8}\right)-\frac{547}{139400} \sigma_{7}\left(\frac{n}{12}\right)+\frac{17504}{17425} \sigma_{7}\left(\frac{n}{24}\right)-\frac{94244213}{20930400} a_{1}(n) \\
& -\frac{1723621}{15390} a_{1}\left(\frac{n}{2}\right)-\frac{17309103}{258400} a_{1}\left(\frac{n}{3}\right)+\frac{416839168}{654075} a_{1}\left(\frac{n}{4}\right)-\frac{92661}{190} a_{1}\left(\frac{n}{6}\right)+\frac{123748608}{8075} a_{1}\left(\frac{n}{12}\right) \\
& +\frac{2319703}{2804400} \Delta_{3,8}(n)-\frac{44249777}{467400} \Delta_{3,8}\left(\frac{n}{2}\right)-\frac{118060434}{19475} \Delta_{3,8}\left(\frac{n}{4}\right)-\frac{2388831616}{175275} \Delta_{3,8}\left(\frac{n}{8}\right) \\
& +\frac{56567}{15390} a_{4}(n)+\frac{176872}{1539} a_{4}\left(\frac{n}{2}\right)+\frac{2243072}{405} a_{4}\left(\frac{n}{4}\right)-\frac{7}{2} a_{2}(n)-\frac{7233}{38} a_{2}\left(\frac{n}{3}\right)+\frac{15}{2} a_{3}(n) \\
& +\frac{7233}{38} a_{3}\left(\frac{n}{3}\right)-\frac{23893}{57} a_{5}(n)-\frac{3256192}{57} a_{5}\left(\frac{n}{2}\right)-\frac{65}{2} a_{6}(n)-\frac{38400}{19} a_{6}\left(\frac{n}{2}\right)-\frac{76688}{57} a_{7}(n) \\
& -\frac{35968}{19} a_{8}(n)+\frac{79232}{19} a_{9}(n) \text {, } \\
& N\left(1^{2}, 3^{2}, 6^{12} ; n\right)=\frac{1}{26764800} \sigma_{7}(n)-\frac{1}{26764800} \sigma_{7}\left(\frac{n}{2}\right)-\frac{547}{8921600} \sigma_{7}\left(\frac{n}{3}\right)+\frac{1}{418200} \sigma_{7}\left(\frac{n}{4}\right) \\
& +\frac{547}{8921600} \sigma_{7}\left(\frac{n}{6}\right)-\frac{32}{52275} \sigma_{7}\left(\frac{n}{8}\right)-\frac{547}{139400} \sigma_{7}\left(\frac{n}{12}\right)+\frac{17504}{17425} \sigma_{7}\left(\frac{n}{24}\right)-\frac{153792059}{31395600} a_{1}(n) \\
& -\frac{85099}{855} a_{1}\left(\frac{n}{2}\right)-\frac{7136643}{129200} a_{1}\left(\frac{n}{3}\right)+\frac{428464448}{654075} a_{1}\left(\frac{n}{4}\right)-\frac{12351}{95} a_{1}\left(\frac{n}{6}\right)+\frac{144295488}{8075} a_{1}\left(\frac{n}{12}\right) \\
& +\frac{2870179}{4206600} \Delta_{3,8}(n)-\frac{203632883}{2103300} \Delta_{3,8}\left(\frac{n}{2}\right)-\frac{833224756}{175275} \Delta_{3,8}\left(\frac{n}{4}\right)-\frac{2034565376}{175275} \Delta_{3,8}\left(\frac{n}{8}\right) \\
& +\frac{97331}{23085} a_{4}(n)+\frac{16912}{171} a_{4}\left(\frac{n}{2}\right)+\frac{1825792}{405} a_{4}\left(\frac{n}{4}\right)-4 a_{2}(n)-\frac{3822}{19} a_{2}\left(\frac{n}{3}\right)+8 a_{3}(n) \\
& +\frac{3594}{19} a_{3}\left(\frac{n}{3}\right)-\frac{3062}{19} a_{5}(n)-\frac{2668160}{57} a_{5}\left(\frac{n}{2}\right)-33 a_{6}(n)-\frac{30912}{19} a_{6}\left(\frac{n}{2}\right) \\
& -\frac{75616}{57} a_{7}(n)-\frac{29696}{19} a_{8}(n)+\frac{73984}{19} a_{9}(n) \text {, } \\
& N\left(1^{2}, 3^{4}, 6^{10} ; n\right)=\frac{1}{13382400} \sigma_{7}(n)-\frac{1}{13382400} \sigma_{7}\left(\frac{n}{2}\right)-\frac{547}{4460800} \sigma_{7}\left(\frac{n}{3}\right)+\frac{1}{418200} \sigma_{7}\left(\frac{n}{4}\right) \\
& +\frac{547}{4460800} \sigma_{7}\left(\frac{n}{6}\right)-\frac{32}{52275} \sigma_{7}\left(\frac{n}{8}\right)-\frac{547}{139400} \sigma_{7}\left(\frac{n}{12}\right)+\frac{17504}{17425} \sigma_{7}\left(\frac{n}{24}\right)-\frac{80615219}{15697800} a_{1}(n) \\
& -\frac{257936}{2565} a_{1}\left(\frac{n}{2}\right)-\frac{3225963}{64600} a_{1}\left(\frac{n}{3}\right)+\frac{430488128}{654075} a_{1}\left(\frac{n}{4}\right)-\frac{5448}{95} a_{1}\left(\frac{n}{6}\right)+\frac{141259968}{8075} a_{1}\left(\frac{n}{12}\right) \\
& +\frac{1298239}{2103300} \Delta_{3,8}(n)-\frac{104642483}{1051650} \Delta_{3,8}\left(\frac{n}{2}\right)-\frac{269811092}{58425} \Delta_{3,8}\left(\frac{n}{4}\right)-\frac{2039918336}{175275} \Delta_{3,8}\left(\frac{n}{8}\right) \\
& +\frac{104302}{23085} a_{4}(n)+\frac{50320}{513} a_{4}\left(\frac{n}{2}\right)+\frac{1795072}{405} a_{4}\left(\frac{n}{4}\right)-4 a_{2}(n)-\frac{3690}{19} a_{2}\left(\frac{n}{3}\right)+8 a_{3}(n) \\
& +\frac{3234}{19} a_{3}\left(\frac{n}{3}\right)-\frac{4420}{57} a_{5}(n)-\frac{2615552}{57} a_{5}\left(\frac{n}{2}\right)-34 a_{6}(n)-\frac{30272}{19} a_{6}\left(\frac{n}{2}\right) \\
& -\frac{77920}{57} a_{7}(n)-\frac{29056}{19} a_{8}(n)+\frac{75136}{19} a_{9}(n) \text {, }
\end{aligned}
$$




$$
\begin{aligned}
& N\left(1^{2}, 3^{6}, 6^{8} ; n\right)=\frac{1}{6691200} \sigma_{7}(n)-\frac{1}{6691200} \sigma_{7}\left(\frac{n}{2}\right)-\frac{547}{2230400} \sigma_{7}\left(\frac{n}{3}\right)+\frac{1}{418200} \sigma_{7}\left(\frac{n}{4}\right) \\
& +\frac{547}{2230400} \sigma_{7}\left(\frac{n}{6}\right)-\frac{32}{52275} \sigma_{7}\left(\frac{n}{8}\right)-\frac{547}{139400} \sigma_{7}\left(\frac{n}{12}\right)+\frac{17504}{17425} \sigma_{7}\left(\frac{n}{24}\right)-\frac{42532669}{7848900} a_{1}(n) \\
& -\frac{263156}{2565} a_{1}\left(\frac{n}{2}\right)+-\frac{1463913}{32300} a_{1}\left(\frac{n}{3}\right)+\frac{145113536}{218025} a_{1}\left(\frac{n}{4}\right)-\frac{588}{95} a_{1}\left(\frac{n}{6}\right)+\frac{137082048}{8075} a_{1}\left(\frac{n}{12}\right) \\
& +\frac{294982}{525825} \Delta_{3,8}(n)-\frac{55481638}{525825} \Delta_{3,8}\left(\frac{n}{2}\right)-\frac{794388736}{175275} \Delta_{3,8}\left(\frac{n}{4}\right)-\frac{686261632}{58425} \Delta_{3,8}\left(\frac{n}{8}\right) \\
& +\frac{112144}{23085} a_{4}(n)+\frac{51712}{513} a_{4}\left(\frac{n}{2}\right)+\frac{592384}{135} a_{4}\left(\frac{n}{4}\right)-4 a_{2}(n)-\frac{3588}{19} a_{2}\left(\frac{n}{3}\right)+8 a_{3}(n) \\
& +\frac{2904}{19} a_{3}\left(\frac{n}{3}\right)-\frac{1112}{57} a_{5}(n)-\frac{860032}{19} a_{5}\left(\frac{n}{2}\right)-36 a_{6}(n)-\frac{29888}{19} a_{6}\left(\frac{n}{2}\right) \\
& -\frac{26880}{19} a_{7}(n)-\frac{28672}{19} a_{8}(n)+\frac{76800}{19} a_{9}(n) \text {, } \\
& N\left(1^{2}, 3^{8}, 6^{6} ; n\right)=\frac{1}{3345600} \sigma_{7}(n)-\frac{1}{3345600} \sigma_{7}\left(\frac{n}{2}\right)-\frac{547}{1115200} \sigma_{7}\left(\frac{n}{3}\right)+\frac{1}{418200} \sigma_{7}\left(\frac{n}{4}\right) \\
& +\frac{547}{1115200} \sigma_{7}\left(\frac{n}{6}\right)-\frac{32}{52275} \sigma_{7}\left(\frac{n}{8}\right)-\frac{547}{139400} \sigma_{7}\left(\frac{n}{12}\right)+\frac{17504}{17425} \sigma_{7}\left(\frac{n}{24}\right)-\frac{1264268}{218025} a_{1}(n) \\
& -\frac{816196}{7695} a_{1}\left(\frac{n}{2}\right)-\frac{340149}{8075} a_{1}\left(\frac{n}{3}\right)+\frac{443021888}{654075} a_{1}\left(\frac{n}{4}\right)+\frac{3084}{95} a_{1}\left(\frac{n}{6}\right)+\frac{131761728}{8075} a_{1}\left(\frac{n}{12}\right) \\
& +\frac{20369}{38950} \Delta_{3,8}(n)-\frac{20689507}{175275} \Delta_{3,8}\left(\frac{n}{2}\right)-\frac{87756104}{19475} \Delta_{3,8}\left(\frac{n}{4}\right)-\frac{2091165056}{175275} \Delta_{3,8}\left(\frac{n}{8}\right) \\
& +\frac{13532}{2565} a_{4}(n)+\frac{168992}{1539} a_{4}\left(\frac{n}{2}\right)+\frac{1772032}{405} a_{4}\left(\frac{n}{4}\right)-4 a_{2}(n)-\frac{3516}{19} a_{2}\left(\frac{n}{3}\right)+8 a_{3}(n) \\
& +\frac{2604}{19} a_{3}\left(\frac{n}{3}\right)-\frac{896}{57} a_{5}(n)-\frac{2561792}{57} a_{5}\left(\frac{n}{2}\right)-40 a_{6}(n)-\frac{29760}{19} a_{6}\left(\frac{n}{2}\right)-\frac{83776}{57} a_{7}(n) \\
& -\frac{28544}{19} a_{8}(n)+\frac{78976}{19} a_{9}(n) \text {, } \\
& N\left(1^{2}, 3^{10}, 6^{4} ; n\right)=\frac{1}{1672800} \sigma_{7}(n)-\frac{1}{1672800} \sigma_{7}\left(\frac{n}{2}\right)-\frac{547}{557600} \sigma_{7}\left(\frac{n}{3}\right)+\frac{1}{418200} \sigma_{7}\left(\frac{n}{4}\right) \\
& +\frac{547}{557600} \sigma_{7}\left(\frac{n}{6}\right)-\frac{32}{52275} \sigma_{7}\left(\frac{n}{8}\right)-\frac{547}{139400} \sigma_{7}\left(\frac{n}{12}\right)+\frac{17504}{17425} \sigma_{7}\left(\frac{n}{24}\right)-\frac{12474544}{1962225} a_{1}(n) \\
& -\frac{171032}{1539} a_{1}\left(\frac{n}{2}\right)-\frac{335538}{8075} a_{1}\left(\frac{n}{3}\right)+\frac{450703168}{654075} a_{1}\left(\frac{n}{4}\right)+\frac{1488}{19} a_{1}\left(\frac{n}{6}\right)+\frac{126441408}{8075} a_{1}\left(\frac{n}{12}\right) \\
& +\frac{272803}{525825} \Delta_{3,8}(n)-\frac{75469222}{525825} \Delta_{3,8}\left(\frac{n}{2}\right)-\frac{264160472}{58425} \Delta_{3,8}\left(\frac{n}{4}\right)-\frac{2123545216}{175275} \Delta_{3,8}\left(\frac{n}{8}\right) \\
& +\frac{134776}{23085} a_{4}(n)+\frac{200480}{1539} a_{4}\left(\frac{n}{2}\right)+\frac{1766912}{405} a_{4}\left(\frac{n}{4}\right)-4 a_{2}(n)-\frac{3444}{19} a_{2}\left(\frac{n}{3}\right)+8 a_{3}(n) \\
& +\frac{2304}{19} a_{3}\left(\frac{n}{3}\right)-\frac{2304}{19} a_{5}(n)-\frac{2543488}{57} a_{5}\left(\frac{n}{2}\right)-48 a_{6}(n)-\frac{29632}{19} a_{6}\left(\frac{n}{2}\right)-\frac{86912}{57} a_{7}(n) \\
& -\frac{28416}{19} a_{8}(n)+\frac{81152}{19} a_{9}(n) \text {, }
\end{aligned}
$$




$$
\begin{aligned}
& N\left(1^{2}, 3^{12}, 6^{2} ; n\right)=\frac{1}{836400} \sigma_{7}(n)-\frac{1}{836400} \sigma_{7}\left(\frac{n}{2}\right)-\frac{547}{278800} \sigma_{7}\left(\frac{n}{3}\right)+\frac{1}{418200} \sigma_{7}\left(\frac{n}{4}\right) \\
& +\frac{547}{278800} \sigma_{7}\left(\frac{n}{6}\right)-\frac{32}{52275} \sigma_{7}\left(\frac{n}{8}\right)-\frac{547}{139400} \sigma_{7}\left(\frac{n}{12}\right)+\frac{17504}{17425} \sigma_{7}\left(\frac{n}{24}\right) \\
& -\frac{14012138}{1962225} a_{1}(n)-\frac{181672}{1539} a_{1}\left(\frac{n}{2}\right)-\frac{454326}{8075} a_{1}\left(\frac{n}{3}\right)+\frac{450703168}{654075} a_{1}\left(\frac{n}{4}\right) \\
& +\frac{576}{19} a_{1}\left(\frac{n}{6}\right)+\frac{126441408}{8075} a_{1}\left(\frac{n}{12}\right)+\frac{293456}{525825} \Delta_{3,8}(n)-\frac{102451844}{525825} \Delta_{3,8}\left(\frac{n}{2}\right) \\
& -\frac{819902216}{175275} \Delta_{3,8}\left(\frac{n}{4}\right)-\frac{2123545216}{175275} \Delta_{3,8}\left(\frac{n}{8}\right)+\frac{151952}{23085} a_{4}(n) \\
& +\frac{268576}{1539} a_{4}\left(\frac{n}{2}\right)+\frac{1766912}{405} a_{4}\left(\frac{n}{4}\right)-4 a_{2}(n)-\frac{3444}{19} a_{2}\left(\frac{n}{3}\right)+8 a_{3}(n) \\
& +\frac{2304}{19} a_{3}\left(\frac{n}{3}\right)-\frac{29408}{57} a_{5}(n)-\frac{2543488}{57} a_{5}\left(\frac{n}{2}\right)-64 a_{6}(n) \\
& -\frac{29632}{19} a_{6}\left(\frac{n}{2}\right)-\frac{86912}{57} a_{7}(n)-\frac{28416}{19} a_{8}(n)+\frac{81152}{19} a_{9}(n) \text {, } \\
& N\left(1^{2}, 3^{14} ; n\right)=\frac{1}{418200} \sigma_{7}(n)-\frac{547}{139400} \sigma_{7}\left(\frac{n}{3}\right)-\frac{32}{52275} \sigma_{7}\left(\frac{n}{4}\right) \\
& +\frac{17504}{17425} \sigma_{7}\left(\frac{n}{12}\right)-\frac{527024}{103275} a_{1}(n)-\frac{818048}{34425} a_{1}\left(\frac{n}{2}\right)-\frac{78048}{425} a_{1}\left(\frac{n}{3}\right) \\
& +\frac{93312}{425} a_{1}\left(\frac{n}{6}\right)+\frac{34688}{27675} \Delta_{3,8}(n)-\frac{285424}{675} \Delta_{3,8}\left(\frac{n}{2}\right)-\frac{6682624}{9225} \Delta_{3,8}\left(\frac{n}{4}\right) \\
& +\frac{9536}{1215} \Delta_{3,8}\left(\frac{n}{8}\right)+\frac{131072}{405} a_{4}(n)-\frac{9472}{3} a_{3}\left(\frac{n}{3}\right)-128 a_{5}\left(\frac{n}{2}\right), \\
& N\left(1^{2}, 2^{2}, 6^{12} ; n\right)=\frac{1}{26764800} \sigma_{7}(n)-\frac{1}{26764800} \sigma_{7}\left(\frac{n}{2}\right)+\frac{273}{8921600} \sigma_{7}\left(\frac{n}{3}\right) \\
& -\frac{1}{209100} \sigma_{7}\left(\frac{n}{4}\right)-\frac{273}{8921600} \sigma_{7}\left(\frac{n}{6}\right)+\frac{64}{52275} \sigma_{7}\left(\frac{n}{8}\right)-\frac{273}{69700} \sigma_{7}\left(\frac{n}{12}\right) \\
& +\frac{17472}{17425} \sigma_{7}\left(\frac{n}{24}\right)-\frac{153792059}{31395600} a_{1}(n)-\frac{2173793}{23085} a_{1}\left(\frac{n}{2}\right)-\frac{7142343}{129200} a_{1}\left(\frac{n}{3}\right) \\
& +\frac{465687424}{654075} a_{1}\left(\frac{n}{4}\right)-\frac{7221}{95} a_{1}\left(\frac{n}{6}\right)+\frac{154681344}{8075} a_{1}\left(\frac{n}{12}\right)+\frac{2870179}{4206600} \Delta_{3,8}(n) \\
& -\frac{7391929}{77900} \Delta_{3,8}\left(\frac{n}{2}\right)-\frac{2395302404}{525825} \Delta_{3,8}\left(\frac{n}{4}\right)-\frac{1827154688}{175275} \Delta_{3,8}\left(\frac{n}{8}\right) \\
& +\frac{97331}{23085} a_{4}(n)+\frac{441424}{4617} a_{4}\left(\frac{n}{2}\right)+\frac{1750016}{405} a_{4}\left(\frac{n}{4}\right)-4 a_{2}(n)-\frac{3879}{19} a_{2}\left(\frac{n}{3}\right) \\
& +8 a_{3}(n)+\frac{3879}{19} a_{3}\left(\frac{n}{3}\right)-\frac{3062}{19} a_{5}(n)-\frac{864256}{19} a_{5}\left(\frac{n}{2}\right)-33 a_{6}(n)-\frac{29696}{19} a_{6}\left(\frac{n}{2}\right) \\
& -\frac{75616}{57} a_{7}(n)-\frac{29696}{19} a_{8}(n)+\frac{73984}{19} a_{9}(n) \text {, }
\end{aligned}
$$




$$
\begin{aligned}
& N\left(1^{2}, 2^{2}, 3^{2}, 6^{10} ; n\right)=\frac{1}{13382400} \sigma_{7}(n)-\frac{1}{13382400} \sigma_{7}\left(\frac{n}{2}\right)+\frac{273}{4460800} \sigma_{7}\left(\frac{n}{3}\right)-\frac{1}{209100} \sigma_{7}\left(\frac{n}{4}\right) \\
& -\frac{273}{4460800} \sigma_{7}\left(\frac{n}{6}\right)+\frac{64}{52275} \sigma_{7}\left(\frac{n}{8}\right)-\frac{273}{69700} \sigma_{7}\left(\frac{n}{12}\right)+\frac{17472}{17425} \sigma_{7}\left(\frac{n}{24}\right)-\frac{6995371}{1744200} a_{1}(n) \\
& -\frac{856144}{7695} a_{1}\left(\frac{n}{2}\right)-\frac{4998303}{64600} a_{1}\left(\frac{n}{3}\right)+\frac{469430144}{654075} a_{1}\left(\frac{n}{4}\right)-\frac{71064}{95} a_{1}\left(\frac{n}{6}\right)+\frac{108756864}{8075} a_{1}\left(\frac{n}{12}\right) \\
& +\frac{223151}{233700} \Delta_{3,8}(n)-\frac{29940601}{350550} \Delta_{3,8}\left(\frac{n}{2}\right)-\frac{382189016}{58425} \Delta_{3,8}\left(\frac{n}{4}\right)-\frac{2269193728}{175275} \Delta_{3,8}\left(\frac{n}{8}\right) \\
& +\frac{7838}{2565} a_{4}(n)+\frac{178976}{1539} a_{4}\left(\frac{n}{2}\right)+\frac{2369536}{405} a_{4}\left(\frac{n}{4}\right)-3 a_{2}(n)-\frac{3717}{19} a_{2}\left(\frac{n}{3}\right)+7 a_{3}(n) \\
& +\frac{4401}{19} a_{3}\left(\frac{n}{3}\right)-\frac{11716}{19} a_{5}(n)-\frac{1156352}{19} a_{5}\left(\frac{n}{2}\right)-30 a_{6}(n)-\frac{40960}{19} a_{6}\left(\frac{n}{2}\right) \\
& -\frac{25024}{19} a_{7}(n)-\frac{40960}{19} a_{8}(n)+\frac{81920}{19} a_{9}(n) \\
& N\left(1^{2}, 2^{2}, 3^{4}, 6^{8} ; n\right)=\frac{1}{6691200} \sigma_{7}(n)-\frac{1}{6691200} \sigma_{7}\left(\frac{n}{2}\right)+\frac{273}{2230400} \sigma_{7}\left(\frac{n}{3}\right)-\frac{1}{209100} \sigma_{7}\left(\frac{n}{4}\right) \\
& -\frac{273}{2230400} \sigma_{7}\left(\frac{n}{6}\right)+\frac{64}{52275} \sigma_{7}\left(\frac{n}{8}\right)-\frac{273}{69700} \sigma_{7}\left(\frac{n}{12}\right)+\frac{17472}{17425} \sigma_{7}\left(\frac{n}{24}\right)-\frac{24875789}{7848900} a_{1}(n) \\
& -\frac{2825864}{23085} a_{1}\left(\frac{n}{2}\right)-\frac{3236253}{32300} a_{1}\left(\frac{n}{3}\right)+\frac{509903744}{654075} a_{1}\left(\frac{n}{4}\right)-\frac{120648}{95} a_{1}\left(\frac{n}{6}\right)+\frac{66651264}{8075} a_{1}\left(\frac{n}{12}\right) \\
& +\frac{650042}{525825} \Delta_{3,8}(n)-\frac{13886026}{175275} \Delta_{3,8}\left(\frac{n}{2}\right)-\frac{4288948904}{525825} \Delta_{3,8}\left(\frac{n}{4}\right)-\frac{2495907328}{175275} \Delta_{3,8}\left(\frac{n}{8}\right) \\
& +\frac{44624}{23085} a_{4}(n)+\frac{616480}{4617} a_{4}\left(\frac{n}{2}\right)+\frac{2861056}{405} a_{4}\left(\frac{n}{4}\right)-2 a_{2}(n)-\frac{3528}{19} a_{2}\left(\frac{n}{3}\right)+6 a_{3}(n) \\
& +\frac{4896}{19} a_{3}\left(\frac{n}{3}\right)-\frac{20856}{19} a_{5}(n)-\frac{1394176}{19} a_{5}\left(\frac{n}{2}\right)-28 a_{6}(n)-\frac{50048}{19} a_{6}\left(\frac{n}{2}\right)-\frac{74944}{57} a_{7}(n) \\
& -\frac{52480}{19} a_{8}(n)+\frac{90368}{19} a_{9}(n) \text {, } \\
& N\left(1^{2}, 2^{2}, 3^{6}, 6^{6} ; n\right)=\frac{1}{3345600} \sigma_{7}(n)-\frac{1}{3345600} \sigma_{7}\left(\frac{n}{2}\right)+\frac{273}{1115200} \sigma_{7}\left(\frac{n}{3}\right)-\frac{1}{209100} \sigma_{7}\left(\frac{n}{4}\right) \\
& -\frac{273}{1115200} \sigma_{7}\left(\frac{n}{6}\right)+\frac{64}{52275} \sigma_{7}\left(\frac{n}{8}\right)-\frac{273}{69700} \sigma_{7}\left(\frac{n}{12}\right)+\frac{17472}{17425} \sigma_{7}\left(\frac{n}{24}\right)-\frac{3672652}{1962225} a_{1}(n) \\
& -\frac{3398428}{23085} a_{1}\left(\frac{n}{2}\right)-\frac{1081479}{8075} a_{1}\left(\frac{n}{3}\right)+\frac{595529344}{654075} a_{1}\left(\frac{n}{4}\right)-\frac{200796}{95} a_{1}\left(\frac{n}{6}\right) \\
& -\frac{21476736}{8075} a_{1}\left(\frac{n}{12}\right)+\frac{1737323}{1051650} \Delta_{3,8}(n)-\frac{3817949}{58425} \Delta_{3,8}\left(\frac{n}{2}\right)-\frac{5906420224}{525825} \Delta_{3,8}\left(\frac{n}{4}\right) \\
& -\frac{2967177728}{175275} \Delta_{3,8}\left(\frac{n}{8}\right)+\frac{5068}{23085} a_{4}(n)+\frac{745472}{4617} a_{4}\left(\frac{n}{2}\right)+\frac{3823616}{405} a_{4}\left(\frac{n}{4}\right)-\frac{2520}{19} a_{2}\left(\frac{n}{3}\right) \\
& +4 a_{3}(n)+\frac{4572}{19} a_{3}\left(\frac{n}{3}\right)-\frac{35200}{19} a_{5}(n)-\frac{1856768}{19} a_{5}\left(\frac{n}{2}\right)-24 a_{6}(n)-\frac{67712}{19} a_{6}\left(\frac{n}{2}\right) \\
& -\frac{81152}{57} a_{7}(n)-\frac{75008}{19} a_{8}(n)+\frac{111104}{19} a_{9}(n) \text {, }
\end{aligned}
$$




$$
\begin{aligned}
& N\left(1^{2}, 2^{2}, 3^{8}, 6^{4} ; n\right)=\frac{1}{1672800} \sigma_{7}(n)-\frac{1}{1672800} \sigma_{7}\left(\frac{n}{2}\right)+\frac{273}{557600} \sigma_{7}\left(\frac{n}{3}\right)-\frac{1}{209100} \sigma_{7}\left(\frac{n}{4}\right) \\
& -\frac{273}{557600} \sigma_{7}\left(\frac{n}{6}\right)+\frac{64}{52275} \sigma_{7}\left(\frac{n}{8}\right)-\frac{273}{69700} \sigma_{7}\left(\frac{n}{12}\right)+\frac{17472}{17425} \sigma_{7}\left(\frac{n}{24}\right)+\frac{691616}{1962225} a_{1}(n) \\
& -\frac{4583032}{23085} a_{1}\left(\frac{n}{2}\right)-\frac{1528518}{8075} a_{1}\left(\frac{n}{3}\right)+\frac{255818368}{218025} a_{1}\left(\frac{n}{4}\right)-\frac{345624}{95} a_{1}\left(\frac{n}{6}\right) \\
& -\frac{201845376}{8075} a_{1}\left(\frac{n}{12}\right)+\frac{1227283}{525825} \Delta_{3,8}(n)-\frac{6525734}{175275} \Delta_{3,8}\left(\frac{n}{2}\right)-\frac{9014649904}{525825} \Delta_{3,8}\left(\frac{n}{4}\right) \\
& -\frac{434057472}{19475} \Delta_{3,8}\left(\frac{n}{8}\right)-\frac{62024}{23085} a_{4}(n)+\frac{976064}{4617} a_{4}\left(\frac{n}{2}\right)+\frac{1897472}{135} a_{4}\left(\frac{n}{4}\right)+4 a_{2}(n) \\
& +\frac{72}{19} a_{2}\left(\frac{n}{3}\right)+\frac{2664}{19} a_{3}\left(\frac{n}{3}\right)-\frac{59968}{19} a_{5}(n)-\frac{2750976}{19} a_{5}\left(\frac{n}{2}\right)-16 a_{6}(n) \\
& -\frac{102016}{19} a_{6}\left(\frac{n}{2}\right)-\frac{99200}{57} a_{7}(n)-\frac{119040}{19} a_{8}(n)+\frac{155392}{19} a_{9}(n) \text {, } \\
& N\left(1^{2}, 2^{2}, 3^{10}, 6^{2} ; n\right)=\frac{1}{836400} \sigma_{7}(n)-\frac{1}{836400} \sigma_{7}\left(\frac{n}{2}\right)+\frac{273}{278800} \sigma_{7}\left(\frac{n}{3}\right)-\frac{1}{209100} \sigma_{7}\left(\frac{n}{4}\right) \\
& -\frac{273}{278800} \sigma_{7}\left(\frac{n}{6}\right)+\frac{64}{52275} \sigma_{7}\left(\frac{n}{8}\right)-\frac{273}{69700} \sigma_{7}\left(\frac{n}{12}\right)+\frac{17472}{17425} \sigma_{7}\left(\frac{n}{24}\right)+\frac{2828554}{654075} a_{1}(n) \\
& -\frac{786488}{2565} a_{1}\left(\frac{n}{2}\right)-\frac{2337426}{8075} a_{1}\left(\frac{n}{3}\right)+\frac{375237248}{218025} a_{1}\left(\frac{n}{4}\right)-\frac{631224}{95} a_{1}\left(\frac{n}{6}\right)-\frac{571787136}{8075} a_{1}\left(\frac{n}{12}\right) \\
& +\frac{625352}{175275} \Delta_{3,8}(n)+\frac{3311632}{175275} \Delta_{3,8}\left(\frac{n}{2}\right)-\frac{1689162416}{58425} \Delta_{3,8}\left(\frac{n}{4}\right)-\frac{1949355776}{58425} \Delta_{3,8}\left(\frac{n}{8}\right) \\
& -\frac{60736}{7695} a_{4}(n)+\frac{157888}{513} a_{4}\left(\frac{n}{2}\right)+\frac{3146752}{135} a_{4}\left(\frac{n}{4}\right)+12 a_{2}(n)+\frac{5796}{19} a_{2}\left(\frac{n}{3}\right)-8 a_{3}(n) \\
& -\frac{2376}{19} a_{3}\left(\frac{n}{3}\right)-\frac{105088}{19} a_{5}(n)+-\frac{4538624}{19} a_{5}\left(\frac{n}{2}\right)-\frac{170880}{19} a_{6}\left(\frac{n}{2}\right)-\frac{47872}{19} a_{7}(n) \\
& -\frac{207360}{19} a_{8}(n)+\frac{249344}{19} a_{9}(n) \\
& N\left(1^{2}, 2^{2}, 3^{12} ; n\right)=\frac{1}{418200} \sigma_{7}(n)-\frac{1}{418200} \sigma_{7}\left(\frac{n}{2}\right)+\frac{273}{139400} \sigma_{7}\left(\frac{n}{3}\right)-\frac{1}{209100} \sigma_{7}\left(\frac{n}{4}\right) \\
& -\frac{273}{139400} \sigma_{7}\left(\frac{n}{6}\right)+\frac{64}{52275} \sigma_{7}\left(\frac{n}{8}\right)-\frac{273}{69700} \sigma_{7}\left(\frac{n}{12}\right)+\frac{17472}{17425} \sigma_{7}\left(\frac{n}{24}\right)+\frac{28625504}{1962225} a_{1}(n) \\
& -\frac{9789472}{23085} a_{1}\left(\frac{n}{2}\right)-\frac{4299492}{8075} a_{1}\left(\frac{n}{3}\right)+\frac{17986944}{8075} a_{1}\left(\frac{n}{4}\right)-\frac{1125024}{95} a_{1}\left(\frac{n}{6}\right)-\frac{1496543616}{8075} a_{1}\left(\frac{n}{12}\right) \\
& +\frac{3450352}{525825} \Delta_{3,8}(n)+\frac{86296}{19475} \Delta_{3,8}\left(\frac{n}{2}\right)-\frac{25061466064}{525825} \Delta_{3,8}\left(\frac{n}{4}\right)-\frac{2627712256}{58425} \Delta_{3,8}\left(\frac{n}{8}\right) \\
& -\frac{395936}{23085} a_{4}(n)+\frac{2531648}{4617} a_{4}\left(\frac{n}{2}\right)+\frac{560128}{15} a_{4}\left(\frac{n}{4}\right)+32 a_{2}(n)+\frac{21672}{19} a_{2}\left(\frac{n}{3}\right) \\
& -32 a_{3}(n)-\frac{17568}{19} a_{3}\left(\frac{n}{3}\right)-\frac{218944}{19} a_{5}(n)-\frac{7209728}{19} a_{5}\left(\frac{n}{2}\right)-\frac{277632}{19} a_{6}\left(\frac{n}{2}\right) \\
& -\frac{171776}{57} a_{7}(n)-\frac{357888}{19} a_{8}(n)+\frac{375296}{19} a_{9}(n) \text {, }
\end{aligned}
$$




$$
\begin{aligned}
& N\left(1^{2}, 2^{4}, 6^{10} ; n\right)=\frac{7}{53529600} \sigma_{7}(n)-\frac{7}{53529600} \sigma_{7}\left(\frac{n}{2}\right)-\frac{549}{17843200} \sigma_{7}\left(\frac{n}{3}\right)+\frac{7}{418200} \sigma_{7}\left(\frac{n}{4}\right) \\
& +\frac{549}{17843200} \sigma_{7}\left(\frac{n}{6}\right)-\frac{224}{52275} \sigma_{7}\left(\frac{n}{8}\right)-\frac{549}{139400} \sigma_{7}\left(\frac{n}{12}\right)+\frac{17568}{17425} \sigma_{7}\left(\frac{n}{24}\right)-\frac{108795931}{20930400} a_{1}(n) \\
& -\frac{1565107}{15390} a_{1}\left(\frac{n}{2}\right)-\frac{10885761}{258400} a_{1}\left(\frac{n}{3}\right)+\frac{121714432}{218025} a_{1}\left(\frac{n}{4}\right)-\frac{91467}{190} a_{1}\left(\frac{n}{6}\right)+\frac{154123776}{8075} a_{1}\left(\frac{n}{12}\right) \\
& +\frac{1459061}{2804400} \Delta_{3,8}(n)-\frac{42635899}{467400} \Delta_{3,8}\left(\frac{n}{2}\right)-\frac{787395782}{175275} \Delta_{3,8}\left(\frac{n}{4}\right)-\frac{703936384}{58425} \Delta_{3,8}\left(\frac{n}{8}\right) \\
& +\frac{71989}{15390} a_{4}(n)+\frac{153016}{1539} a_{4}\left(\frac{n}{2}\right)+\frac{578048}{135} a_{4}\left(\frac{n}{4}\right)-\frac{9}{2} a_{2}(n)-\frac{8667}{38} a_{2}\left(\frac{n}{3}\right)+\frac{17}{2} a_{3}(n) \\
& +\frac{8667}{38} a_{3}\left(\frac{n}{3}\right)+\frac{2991}{19} a_{5}(n)-\frac{838272}{19} a_{5}\left(\frac{n}{2}\right)-\frac{63}{2} a_{6}(n)-\frac{29440}{19} a_{6}\left(\frac{n}{2}\right)-\frac{23952}{19} a_{7}(n) \\
& -\frac{22144}{19} a_{8}(n)+\frac{66176}{19} a_{9}(n) \text {, } \\
& N\left(1^{2}, 2^{4}, 3^{2}, 6^{8} ; n\right)=\frac{7}{26764800} \sigma_{7}(n)-\frac{7}{26764800} \sigma_{7}\left(\frac{n}{2}\right)-\frac{549}{8921600} \sigma_{7}\left(\frac{n}{3}\right)+\frac{7}{418200} \sigma_{7}\left(\frac{n}{4}\right) \\
& +\frac{549}{8921600} \sigma_{7}\left(\frac{n}{6}\right)-\frac{224}{52275} \sigma_{7}\left(\frac{n}{8}\right)-\frac{549}{139400} \sigma_{7}\left(\frac{n}{12}\right)+\frac{17568}{17425} \sigma_{7}\left(\frac{n}{24}\right)-\frac{77749511}{10465200} a_{1}(n) \\
& -\frac{618169}{7695} a_{1}\left(\frac{n}{2}\right)+\frac{1651059}{129200} a_{1}\left(\frac{n}{3}\right)+\frac{341223616}{654075} a_{1}\left(\frac{n}{4}\right)+\frac{90531}{95} a_{1}\left(\frac{n}{6}\right)+\frac{235740096}{8075} a_{1}\left(\frac{n}{12}\right) \\
& -\frac{220709}{1402200} \Delta_{3,8}(n)-\frac{78808147}{701100} \Delta_{3,8}\left(\frac{n}{2}\right)-\frac{186591212}{175275} \Delta_{3,8}\left(\frac{n}{4}\right)-\frac{1540328192}{175275} \Delta_{3,8}\left(\frac{n}{8}\right) \\
& +\frac{58379}{7695} a_{4}(n)+\frac{95984}{1539} a_{4}\left(\frac{n}{2}\right)+\frac{717824}{405} a_{4}\left(\frac{n}{4}\right)-6 a_{2}(n)-\frac{3888}{19} a_{2}\left(\frac{n}{3}\right)+10 a_{3}(n) \\
& +\frac{1836}{19} a_{3}\left(\frac{n}{3}\right)+\frac{22902}{19} a_{5}(n)-\frac{351360}{19} a_{5}\left(\frac{n}{2}\right)-39 a_{6}(n)-\frac{10560}{19} a_{6}\left(\frac{n}{2}\right)-\frac{26848}{19} a_{7}(n) \\
& -\frac{2048}{19} a_{8}(n)+\frac{57600}{19} a_{9}(n) \text {, } \\
& N\left(1^{2}, 2^{4}, 3^{4}, 6^{6} ; n\right)=\frac{7}{13382400} \sigma_{7}(n)-\frac{7}{13382400} \sigma_{7}\left(\frac{n}{2}\right)-\frac{549}{4460800} \sigma_{7}\left(\frac{n}{3}\right)+\frac{7}{418200} \sigma_{7}\left(\frac{n}{4}\right) \\
& +\frac{549}{4460800} \sigma_{7}\left(\frac{n}{6}\right)-\frac{224}{52275} \sigma_{7}\left(\frac{n}{8}\right)-\frac{549}{139400} \sigma_{7}\left(\frac{n}{12}\right)+\frac{17568}{17425} \sigma_{7}\left(\frac{n}{24}\right)-\frac{51387271}{5232600} a_{1}(n) \\
& -\frac{468224}{7695} a_{1}\left(\frac{n}{2}\right)+\frac{4784499}{64600} a_{1}\left(\frac{n}{3}\right)+\frac{29004224}{72675} a_{1}\left(\frac{n}{4}\right)+\frac{210456}{95} a_{1}\left(\frac{n}{6}\right)+\frac{312607296}{8075} a_{1}\left(\frac{n}{12}\right) \\
& -\frac{640549}{701100} \Delta_{3,8}(n)-\frac{15592709}{116850} \Delta_{3,8}\left(\frac{n}{2}\right)+\frac{458100988}{175275} \Delta_{3,8}\left(\frac{n}{4}\right)-\frac{119775488}{19475} \Delta_{3,8}\left(\frac{n}{8}\right) \\
& +\frac{82598}{7695} a_{4}(n)+\frac{39760}{1539} a_{4}\left(\frac{n}{2}\right)-\frac{41984}{45} a_{4}\left(\frac{n}{4}\right)-8 a_{2}(n)-\frac{3726}{19} a_{2}\left(\frac{n}{3}\right)+12 a_{3}(n) \\
& -\frac{378}{19} a_{3}\left(\frac{n}{3}\right)+\frac{46092}{19} a_{5}(n)+\frac{185856}{19} a_{5}\left(\frac{n}{2}\right)-46 a_{6}(n)+\frac{9792}{19} a_{6}\left(\frac{n}{2}\right) \\
& -\frac{28896}{19} a_{7}(n)+\frac{23168}{19} a_{8}(n)+\frac{43648}{19} a_{9}(n) \text {, }
\end{aligned}
$$




$$
\begin{aligned}
& N\left(1^{2}, 2^{4}, 3^{6}, 6^{4} ; n\right)=\frac{7}{6691200} \sigma_{7}(n)-\frac{7}{6691200} \sigma_{7}\left(\frac{n}{2}\right)-\frac{549}{2230400} \sigma_{7}\left(\frac{n}{3}\right)+\frac{7}{418200} \sigma_{7}\left(\frac{n}{4}\right) \\
& +\frac{549}{2230400} \sigma_{7}\left(\frac{n}{6}\right)-\frac{224}{52275} \sigma_{7}\left(\frac{n}{8}\right)-\frac{549}{139400} \sigma_{7}\left(\frac{n}{12}\right)+\frac{17568}{17425} \sigma_{7}\left(\frac{n}{24}\right)-\frac{1286743}{96900} a_{1}(n) \\
& -\frac{26284}{1539} a_{1}\left(\frac{n}{2}\right)+\frac{4994109}{32300} a_{1}\left(\frac{n}{3}\right)+\frac{99034816}{654075} a_{1}\left(\frac{n}{4}\right)+\frac{77076}{19} a_{1}\left(\frac{n}{6}\right)+\frac{478092096}{8075} a_{1}\left(\frac{n}{12}\right) \\
& -\frac{111439}{58425} \Delta_{3,8}(n)-\frac{31565692}{175275} \Delta_{3,8}\left(\frac{n}{2}\right)+\frac{529567136}{58425} \Delta_{3,8}\left(\frac{n}{4}\right)-\frac{167057792}{175275} \Delta_{3,8}\left(\frac{n}{8}\right) \\
& +\frac{4328}{285} a_{4}(n)-\frac{33152}{1539} a_{4}\left(\frac{n}{2}\right)-\frac{2377216}{405} a_{4}\left(\frac{n}{4}\right)-12 a_{2}(n)-\frac{5292}{19} a_{2}\left(\frac{n}{3}\right)+16 a_{3}(n) \\
& -\frac{864}{19} a_{3}\left(\frac{n}{3}\right)+\frac{77928}{19} a_{5}(n)+\frac{1155456}{19} a_{5}\left(\frac{n}{2}\right)-60 a_{6}(n)+\frac{46528}{19} a_{6}\left(\frac{n}{2}\right) \\
& -\frac{27136}{19} a_{7}(n)+\frac{69632}{19} a_{8}(n)+\frac{6656}{19} a_{9}(n) \text {, } \\
& N\left(1^{2}, 2^{4}, 3^{8}, 6^{2} ; n\right)=\frac{7}{3345600} \sigma_{7}(n)-\frac{7}{3345600} \sigma_{7}\left(\frac{n}{2}\right)-\frac{549}{1115200} \sigma_{7}\left(\frac{n}{3}\right)+\frac{7}{418200} \sigma_{7}\left(\frac{n}{4}\right) \\
& +\frac{549}{1115200} \sigma_{7}\left(\frac{n}{6}\right)-\frac{224}{52275} \sigma_{7}\left(\frac{n}{8}\right)-\frac{549}{139400} \sigma_{7}\left(\frac{n}{12}\right)+\frac{17568}{17425} \sigma_{7}\left(\frac{n}{24}\right)-\frac{651532}{34425} a_{1}(n) \\
& +\frac{30428}{405} a_{1}\left(\frac{n}{2}\right)+\frac{115983}{425} a_{1}\left(\frac{n}{3}\right)-\frac{3890752}{11475} a_{1}\left(\frac{n}{4}\right)+\frac{36108}{5} a_{1}\left(\frac{n}{6}\right)+\frac{43308864}{425} a_{1}\left(\frac{n}{12}\right) \\
& -\frac{62107}{18450} \Delta_{3,8}(n)-\frac{281839}{1025} \Delta_{3,8}\left(\frac{n}{2}\right)+\frac{193400312}{9225} \Delta_{3,8}\left(\frac{n}{4}\right)+\frac{9441408}{1025} \Delta_{3,8}\left(\frac{n}{8}\right) \\
& +\frac{9028}{405} a_{4}(n)-\frac{7648}{81} a_{4}\left(\frac{n}{2}\right)-\frac{2043392}{135} a_{4}\left(\frac{n}{4}\right)-20 a_{2}(n)-540 a_{2}\left(\frac{n}{3}\right) \\
& +24 a_{3}(n)+108 a_{3}\left(\frac{n}{3}\right)+6720 a_{5}(n)+155904 a_{5}\left(\frac{n}{2}\right)-88 a_{6}(n) \\
& +6080 a_{6}\left(\frac{n}{2}\right)-960 a_{7}(n)+8320 a_{8}(n)-3968 a_{9}(n), \\
& N\left(1^{2}, 2^{4}, 3^{10} ; n\right)=\frac{7}{1672800} \sigma_{7}(n)-\frac{7}{1672800} \sigma_{7}\left(\frac{n}{2}\right)-\frac{549}{557600} \sigma_{7}\left(\frac{n}{3}\right)+\frac{7}{418200} \sigma_{7}\left(\frac{n}{4}\right) \\
& +\frac{549}{557600} \sigma_{7}\left(\frac{n}{6}\right)-\frac{224}{52275} \sigma_{7}\left(\frac{n}{8}\right)-\frac{549}{139400} \sigma_{7}\left(\frac{n}{12}\right)+\frac{17568}{17425} \sigma_{7}\left(\frac{n}{24}\right)-\frac{6190412}{218025} a_{1}(n) \\
& +\frac{239272}{855} a_{1}\left(\frac{n}{2}\right)+\frac{3835134}{8075} a_{1}\left(\frac{n}{3}\right)-\frac{99923776}{72675} a_{1}\left(\frac{n}{4}\right)+\frac{1284768}{95} a_{1}\left(\frac{n}{6}\right)+\frac{1522898496}{8075} a_{1}\left(\frac{n}{12}\right) \\
& -\frac{114077}{19475} \Delta_{3,8}(n)-\frac{9072342}{19475} \Delta_{3,8}\left(\frac{n}{2}\right)+\frac{2585843416}{58425} \Delta_{3,8}\left(\frac{n}{4}\right)+\frac{591722112}{19475} \Delta_{3,8}\left(\frac{n}{8}\right) \\
& +\frac{87848}{2565} a_{4}(n)-\frac{38432}{171} a_{4}\left(\frac{n}{2}\right)-\frac{1508864}{45} a_{4}\left(\frac{n}{4}\right)-36 a_{2}(n)-\frac{21924}{19} a_{2}\left(\frac{n}{3}\right) \\
& +40 a_{3}(n)+\frac{11664}{19} a_{3}\left(\frac{n}{3}\right)+\frac{208128}{19} a_{5}(n)+\frac{6533760}{19} a_{5}\left(\frac{n}{2}\right)-144 a_{6}(n) \\
& +\frac{252864}{19} a_{6}\left(\frac{n}{2}\right)+\frac{8832}{19} a_{7}(n)+\frac{329472}{19} a_{8}(n)-\frac{255232}{19} a_{9}(n) \text {, }
\end{aligned}
$$




$$
\begin{aligned}
& N\left(1^{2}, 2^{6}, 6^{8} ; n\right)=\frac{1}{2676480} \sigma_{7}(n)-\frac{1}{2676480} \sigma_{7}\left(\frac{n}{2}\right)+\frac{27}{892160} \sigma_{7}\left(\frac{n}{3}\right)-\frac{1}{20910} \sigma_{7}\left(\frac{n}{4}\right) \\
& -\frac{27}{892160} \sigma_{7}\left(\frac{n}{6}\right)+\frac{128}{10455} \sigma_{7}\left(\frac{n}{8}\right)-\frac{27}{6970} \sigma_{7}\left(\frac{n}{12}\right)+\frac{3456}{3485} \sigma_{7}\left(\frac{n}{24}\right)-\frac{61573}{12920} a_{1}(n) \\
& -\frac{9552}{95} a_{1}\left(\frac{n}{2}\right)-\frac{691173}{12920} a_{1}\left(\frac{n}{3}\right)+\frac{10954112}{14535} a_{1}\left(\frac{n}{4}\right)+\frac{11178}{95} a_{1}\left(\frac{n}{6}\right)+\frac{36799488}{1615} a_{1}\left(\frac{n}{12}\right) \\
& +\frac{10287}{15580} \Delta_{3,8}(n)-\frac{118219}{1558} \Delta_{3,8}\left(\frac{n}{2}\right)-\frac{65092856}{11685} \Delta_{3,8}\left(\frac{n}{4}\right)-\frac{132326912}{11685} \Delta_{3,8}\left(\frac{n}{8}\right) \\
& +\frac{78}{19} a_{4}(n)+\frac{1696}{19} a_{4}\left(\frac{n}{2}\right)+\frac{47104}{9} a_{4}\left(\frac{n}{4}\right)-3 a_{2}(n)-\frac{3402}{19} a_{2}\left(\frac{n}{3}\right)+7 a_{3}(n) \\
& +\frac{3402}{19} a_{3}\left(\frac{n}{3}\right)-\frac{3060}{19} a_{5}(n)-\frac{1059840}{19} a_{5}\left(\frac{n}{2}\right)-30 a_{6}(n)+-\frac{35840}{19} a_{6}\left(\frac{n}{2}\right) \\
& -\frac{26304}{19} a_{7}(n)-\frac{35840}{19} a_{8}(n)+\frac{81408}{19} a_{9}(n) \text {, } \\
& N\left(1^{2}, 2^{6}, 3^{2}, 6^{6} ; n\right)=\frac{1}{1338240} \sigma_{7}(n)-\frac{1}{1338240} \sigma_{7}\left(\frac{n}{2}\right)+\frac{27}{446080} \sigma_{7}\left(\frac{n}{3}\right)-\frac{1}{20910} \sigma_{7}\left(\frac{n}{4}\right) \\
& -\frac{27}{446080} \sigma_{7}\left(\frac{n}{6}\right)+\frac{128}{10455} \sigma_{7}\left(\frac{n}{8}\right)-\frac{27}{6970} \sigma_{7}\left(\frac{n}{12}\right)+\frac{3456}{3485} \sigma_{7}\left(\frac{n}{24}\right)+\frac{5129}{11628} a_{1}(n) \\
& -\frac{300662}{2565} a_{1}\left(\frac{n}{2}\right)-\frac{242703}{1292} a_{1}\left(\frac{n}{3}\right)+\frac{34596608}{43605} a_{1}\left(\frac{n}{4}\right)-\frac{281826}{95} a_{1}\left(\frac{n}{6}\right)+\frac{13181184}{1615} a_{1}\left(\frac{n}{12}\right) \\
& +\frac{5419}{2337} \Delta_{3,8}(n)-\frac{495602}{11685} \Delta_{3,8}\left(\frac{n}{2}\right)-\frac{43485456}{3895} \Delta_{3,8}\left(\frac{n}{4}\right)-\frac{174772736}{11685} \Delta_{3,8}\left(\frac{n}{8}\right) \\
& -\frac{472}{171} a_{4}(n)+\frac{84544}{513} a_{4}\left(\frac{n}{2}\right)+\frac{243712}{27} a_{4}\left(\frac{n}{4}\right)-a_{2}(n)-\frac{6723}{19} a_{2}\left(\frac{n}{3}\right)+5 a_{3}(n) \\
& +\frac{12879}{19} a_{3}\left(\frac{n}{3}\right)-\frac{48960}{19} a_{5}(n)-\frac{1810944}{19} a_{5}\left(\frac{n}{2}\right)-16 a_{6}(n)-\frac{65152}{19} a_{6}\left(\frac{n}{2}\right) \\
& -\frac{12288}{19} a_{7}(n)-\frac{67584}{19} a_{8}(n)+\frac{76800}{19} a_{9}(n), \\
& N\left(1^{2}, 2^{6}, 3^{4}, 6^{4} ; n\right)=\frac{1}{669120} \sigma_{7}(n)-\frac{1}{669120} \sigma_{7}\left(\frac{n}{2}\right)+\frac{27}{223040} \sigma_{7}\left(\frac{n}{3}\right)-\frac{1}{20910} \sigma_{7}\left(\frac{n}{4}\right) \\
& -\frac{27}{223040} \sigma_{7}\left(\frac{n}{6}\right)+\frac{128}{10455} \sigma_{7}\left(\frac{n}{8}\right)-\frac{27}{6970} \sigma_{7}\left(\frac{n}{12}\right)+\frac{3456}{3485} \sigma_{7}\left(\frac{n}{24}\right)+\frac{265528}{43605} a_{1}(n) \\
& -\frac{69688}{513} a_{1}\left(\frac{n}{2}\right)+-\frac{538596}{1615} a_{1}\left(\frac{n}{3}\right)+\frac{42055936}{43605} a_{1}\left(\frac{n}{4}\right)-\frac{106920}{19} a_{1}\left(\frac{n}{6}\right)-\frac{5501952}{1615} a_{1}\left(\frac{n}{12}\right) \\
& +\frac{96203}{23370} \Delta_{3,8}(n)+\frac{48679}{11685} \Delta_{3,8}\left(\frac{n}{2}\right)-\frac{207939152}{11685} \Delta_{3,8}\left(\frac{n}{4}\right)-\frac{220493312}{11685} \Delta_{3,8}\left(\frac{n}{8}\right) \\
& -\frac{5236}{513} a_{4}(n)+\frac{119872}{513} a_{4}\left(\frac{n}{2}\right)+\frac{370688}{27} a_{4}\left(\frac{n}{4}\right)+2 a_{2}(n)-\frac{9396}{19} a_{2}\left(\frac{n}{3}\right)+2 a_{3}(n) \\
& +\frac{21708}{19} a_{3}\left(\frac{n}{3}\right)-\frac{100368}{19} a_{5}(n)-\frac{2762496}{19} a_{5}\left(\frac{n}{2}\right)-\frac{100608}{19} a_{6}\left(\frac{n}{2}\right)-\frac{384}{19} a_{7}(n) \\
& -\frac{110336}{19} a_{8}(n)+\frac{84480}{19} a_{9}(n) \text {, }
\end{aligned}
$$




$$
\begin{aligned}
& N\left(1^{2}, 2^{6}, 3^{6}, 6^{2} ; n\right)=\frac{1}{334560} \sigma_{7}(n)-\frac{1}{334560} \sigma_{7}\left(\frac{n}{2}\right)+\frac{27}{111520} \sigma_{7}\left(\frac{n}{3}\right)-\frac{1}{20910} \sigma_{7}\left(\frac{n}{4}\right) \\
& -\frac{27}{111520} \sigma_{7}\left(\frac{n}{6}\right)+\frac{128}{10455} \sigma_{7}\left(\frac{n}{8}\right)-\frac{27}{6970} \sigma_{7}\left(\frac{n}{12}\right)+\frac{3456}{3485} \sigma_{7}\left(\frac{n}{24}\right)+\frac{1205677}{87210} a_{1}(n) \\
& -\frac{153172}{855} a_{1}\left(\frac{n}{2}\right)-\frac{1619649}{3230} a_{1}\left(\frac{n}{3}\right)+\frac{55151104}{43605} a_{1}\left(\frac{n}{4}\right)-\frac{838188}{95} a_{1}\left(\frac{n}{6}\right)-\frac{47685888}{1615} a_{1}\left(\frac{n}{12}\right) \\
& +\frac{144641}{23370} \Delta_{3,8}(n)+\frac{1281397}{11685} \Delta_{3,8}\left(\frac{n}{2}\right)-\frac{327361984}{11685} \Delta_{3,8}\left(\frac{n}{4}\right)-\frac{298908928}{11685} \Delta_{3,8}\left(\frac{n}{8}\right) \\
& -\frac{10268}{513} a_{4}(n)+\frac{50432}{171} a_{4}\left(\frac{n}{2}\right)+\frac{576512}{27} a_{4}\left(\frac{n}{4}\right)+8 a_{2}(n)-\frac{9072}{19} a_{2}\left(\frac{n}{3}\right)-4 a_{3}(n) \\
& +\frac{27540}{19} a_{3}\left(\frac{n}{3}\right)-\frac{162288}{19} a_{5}(n)-\frac{4285440}{19} a_{5}\left(\frac{n}{2}\right)+32 a_{6}(n)-\frac{157184}{19} a_{6}\left(\frac{n}{2}\right) \\
& +\frac{7680}{19} a_{7}(n)-\frac{181504}{19} a_{8}(n)+\frac{119808}{19} a_{9}(n) \text {, } \\
& N\left(1^{2}, 2^{6}, 3^{8} ; n\right)=\frac{1}{167280} \sigma_{7}(n)-\frac{1}{167280} \sigma_{7}\left(\frac{n}{2}\right)+\frac{27}{55760} \sigma_{7}\left(\frac{n}{3}\right)-\frac{1}{20910} \sigma_{7}\left(\frac{n}{4}\right) \\
& -\frac{27}{55760} \sigma_{7}\left(\frac{n}{6}\right)+\frac{128}{10455} \sigma_{7}\left(\frac{n}{8}\right)-\frac{27}{6970} \sigma_{7}\left(\frac{n}{12}\right)+\frac{3456}{3485} \sigma_{7}\left(\frac{n}{24}\right)+\frac{376976}{14535} a_{1}(n) \\
& -\frac{743264}{2565} a_{1}\left(\frac{n}{2}\right)-\frac{1148256}{1615} a_{1}\left(\frac{n}{3}\right)+\frac{16063744}{8721} a_{1}\left(\frac{n}{4}\right)-\frac{1322352}{95} a_{1}\left(\frac{n}{6}\right)-\frac{27350784}{323} a_{1}\left(\frac{n}{12}\right) \\
& +\frac{34178}{3895} \Delta_{3,8}(n)+\frac{765004}{2337} \Delta_{3,8}\left(\frac{n}{2}\right)-\frac{542720224}{11685} \Delta_{3,8}\left(\frac{n}{4}\right)-\frac{29861376}{779} \Delta_{3,8}\left(\frac{n}{8}\right) \\
& -\frac{5936}{171} a_{4}(n)+\frac{188800}{513} a_{4}\left(\frac{n}{2}\right)+\frac{956416}{27} a_{4}\left(\frac{n}{4}\right)+20 a_{2}(n)-\frac{2592}{19} a_{2}\left(\frac{n}{3}\right) \\
& -16 a_{3}(n)+\frac{27216}{19} a_{3}\left(\frac{n}{3}\right)-\frac{248832}{19} a_{5}(n)-\frac{7077888}{19} a_{5}\left(\frac{n}{2}\right)+96 a_{6}(n) \\
& -\frac{262400}{19} a_{6}\left(\frac{n}{2}\right)+\frac{8448}{19} a_{7}(n)-\frac{315904}{19} a_{8}(n)+\frac{213504}{19} a_{9}(n), \\
& N\left(1^{2}, 2^{8}, 6^{6} ; n\right)=\frac{61}{53529600} \sigma_{7}(n)-\frac{61}{53529600} \sigma_{7}\left(\frac{n}{2}\right)-\frac{567}{17843200} \sigma_{7}\left(\frac{n}{3}\right)+\frac{61}{418200} \sigma_{7}\left(\frac{n}{4}\right) \\
& +\frac{567}{17843200} \sigma_{7}\left(\frac{n}{6}\right)-\frac{1952}{52275} \sigma_{7}\left(\frac{n}{8}\right)-\frac{567}{139400} \sigma_{7}\left(\frac{n}{12}\right)+\frac{18144}{17425} \sigma_{7}\left(\frac{n}{24}\right)-\frac{255121}{40800} a_{1}(n) \\
& -\frac{26393}{270} a_{1}\left(\frac{n}{2}\right)-\frac{314577}{13600} a_{1}\left(\frac{n}{3}\right)+\frac{5325824}{11475} a_{1}\left(\frac{n}{4}\right)-\frac{13419}{10} a_{1}\left(\frac{n}{6}\right)+\frac{6296832}{425} a_{1}\left(\frac{n}{12}\right) \\
& +\frac{14059}{49200} \Delta_{3,8}(n)-\frac{3072743}{24600} \Delta_{3,8}\left(\frac{n}{2}\right)-\frac{8486458}{3075} \Delta_{3,8}\left(\frac{n}{4}\right)-\frac{31138688}{3075} \Delta_{3,8}\left(\frac{n}{8}\right) \\
& +\frac{179}{30} a_{4}(n)+\frac{3272}{27} a_{4}\left(\frac{n}{2}\right)+\frac{361984}{135} a_{4}\left(\frac{n}{4}\right)-\frac{11}{2} a_{2}(n)-\frac{567}{2} a_{2}\left(\frac{n}{3}\right)+\frac{19}{2} a_{3}(n) \\
& +\frac{567}{2} a_{3}\left(\frac{n}{3}\right)+567 a_{5}(n)-26496 a_{5}\left(\frac{n}{2}\right)-\frac{77}{2} a_{6}(n)-1024 a_{6}\left(\frac{n}{2}\right)-1104 a_{7}((n) \\
& -384 a_{8}(n)+2688 a_{9}(n) \text {, }
\end{aligned}
$$




$$
\begin{aligned}
& N\left(1^{2}, 2^{8}, 3^{2}, 6^{4} ; n\right)=\frac{61}{26764800} \sigma_{7}(n)-\frac{61}{26764800} \sigma_{7}\left(\frac{n}{2}\right)-\frac{567}{8921600} \sigma_{7}\left(\frac{n}{3}\right)+\frac{61}{418200} \sigma_{7}\left(\frac{n}{4}\right) \\
& +\frac{567}{8921600} \sigma_{7}\left(\frac{n}{6}\right)-\frac{1952}{52275} \sigma_{7}\left(\frac{n}{8}\right)-\frac{567}{139400} \sigma_{7}\left(\frac{n}{12}\right)+\frac{18144}{17425} \sigma_{7}\left(\frac{n}{24}\right)-\frac{208099573}{10465200} a_{1}(n) \\
& -\frac{279413}{2565} a_{1}\left(\frac{n}{2}\right)+\frac{45905337}{129200} a_{1}\left(\frac{n}{3}\right)+\frac{299725888}{654075} a_{1}\left(\frac{n}{4}\right)+\frac{585621}{95} a_{1}\left(\frac{n}{6}\right)+\frac{240913728}{8075} a_{1}\left(\frac{n}{12}\right) \\
& -\frac{6150187}{1402200} \Delta_{3,8}(n)-\frac{111166261}{701100} \Delta_{3,8}\left(\frac{n}{2}\right)+\frac{1456122524}{175275} \Delta_{3,8}\left(\frac{n}{4}\right)-\frac{1105024256}{175275} \Delta_{3,8}\left(\frac{n}{8}\right) \\
& +\frac{186757}{7695} a_{4}(n)-\frac{45200}{513} a_{4}\left(\frac{n}{2}\right)-\frac{1635328}{405} a_{4}\left(\frac{n}{4}\right)-8 a_{2}(n)+\frac{8046}{19} a_{2}\left(\frac{n}{3}\right)+12 a_{3}(n) \\
& -\frac{26514}{19} a_{3}\left(\frac{n}{3}\right)+\frac{138690}{19} a_{5}(n)+\frac{878208}{19} a_{5}\left(\frac{n}{2}\right)-61 a_{6}(n)+\frac{34368}{19} a_{6}\left(\frac{n}{2}\right)-\frac{70304}{19} a_{7}(n) \\
& +\frac{50176}{19} a_{8}(n)+\frac{108800}{19} a_{9}(n) \text {, } \\
& N\left(1^{2}, 2^{8}, 3^{4}, 6^{2} ; n\right)=\frac{61}{13382400} \sigma_{7}(n)-\frac{61}{13382400} \sigma_{7}\left(\frac{n}{2}\right)-\frac{567}{4460800} \sigma_{7}\left(\frac{n}{3}\right)+\frac{61}{418200} \sigma_{7}\left(\frac{n}{4}\right) \\
& +\frac{567}{4460800} \sigma_{7}\left(\frac{n}{6}\right)-\frac{1952}{52275} \sigma_{7}\left(\frac{n}{8}\right)-\frac{567}{139400} \sigma_{7}\left(\frac{n}{12}\right)+\frac{18144}{17425} \sigma_{7}\left(\frac{n}{24}\right)-\frac{178701133}{5232600} a_{1}(n) \\
& -\frac{286448}{2565} a_{1}\left(\frac{n}{2}\right)+\frac{48457377}{64600} a_{1}\left(\frac{n}{3}\right)+\frac{159265088}{654075} a_{1}\left(\frac{n}{4}\right)+\frac{1176696}{95} a_{1}\left(\frac{n}{6}\right)+\frac{290363328}{8075} a_{1}\left(\frac{n}{12}\right) \\
& -\frac{6492127}{701100} \Delta_{3,8}(n)-\frac{83264941}{350550} \Delta_{3,8}\left(\frac{n}{2}\right)+\frac{3806791924}{175275} \Delta_{3,8}\left(\frac{n}{4}\right)-\frac{109478656}{175275} \Delta_{3,8}\left(\frac{n}{8}\right) \\
& +\frac{334034}{7695} a_{4}(n)-\frac{136880}{513} a_{4}\left(\frac{n}{2}\right)-\frac{5270528}{405} a_{4}\left(\frac{n}{4}\right)-12 a_{2}(n)+\frac{20142}{19} a_{2}\left(\frac{n}{3}\right)+16 a_{3}(n) \\
& -\frac{57078}{19} a_{3}\left(\frac{n}{3}\right)+\frac{272196}{19} a_{5}(n)+\frac{2747136}{19} a_{5}\left(\frac{n}{2}\right)-90 a_{6}(n)+\frac{102080}{19} a_{6}\left(\frac{n}{2}\right) \\
& -\frac{114592}{19} a_{7}(n)+\frac{127616}{19} a_{8}(n)+\frac{141184}{19} a_{9}(n) \text {, } \\
& N\left(\left(1^{2}, 2^{8}, 3^{6} ; n\right)=\frac{61}{6691200} \sigma_{7}(n)-\frac{61}{6691200} \sigma_{7}\left(\frac{n}{2}\right)-\frac{567}{2230400} \sigma_{7}\left(\frac{n}{3}\right)+\frac{61}{418200} \sigma_{7}\left(\frac{n}{4}\right)\right. \\
& +\frac{567}{2230400} \sigma_{7}\left(\frac{n}{6}\right)-\frac{1952}{52275} \sigma_{7}\left(\frac{n}{8}\right)-\frac{567}{139400} \sigma_{7}\left(\frac{n}{12}\right)+\frac{18144}{17425} \sigma_{7}\left(\frac{n}{24}\right)-\frac{138641483}{2616300} a_{1}(n) \\
& -\frac{120572}{855} a_{1}\left(\frac{n}{2}\right)+\frac{37020627}{32300} a_{1}\left(\frac{n}{3}\right)+\frac{117290048}{654075} a_{1}\left(\frac{n}{4}\right)+\frac{1664532}{95} a_{1}\left(\frac{n}{6}\right)+\frac{418442688}{8075} a_{1}\left(\frac{n}{12}\right) \\
& -\frac{2479876}{175275} \Delta_{3,8}(n)-\frac{74696966}{175275} \Delta_{3,8}\left(\frac{n}{2}\right)+\frac{6475057984}{175275} \Delta_{3,8}\left(\frac{n}{4}\right)+\frac{523351424}{175275} \Delta_{3,8}\left(\frac{n}{8}\right) \\
& +\frac{516608}{7695} a_{4}(n)-\frac{64256}{171} a_{4}\left(\frac{n}{2}\right)-\frac{9456128}{405} a_{4}\left(\frac{n}{4}\right)-20 a_{2}(n)+\frac{27756}{19} a_{2}\left(\frac{n}{3}\right)+24 a_{3}(n) \\
& -\frac{83160}{19} a_{3}\left(\frac{n}{3}\right)+\frac{422424}{19} a_{5}(n)+\frac{4911744}{19} a_{5}\left(\frac{n}{2}\right)-148 a_{6}(n)+\frac{178240}{19} a_{6}\left(\frac{n}{2}\right) \\
& -\frac{167680}{19} a_{7}(n)+\frac{223232}{19} a_{8}(n)+\frac{176128}{19} a_{9}(n) \text {, }
\end{aligned}
$$




$$
\begin{aligned}
& N\left(1^{2}, 2^{10}, 6^{4} ; n\right)=\frac{91}{26764800} \sigma_{7}(n)-\frac{91}{26764800} \sigma_{7}\left(\frac{n}{2}\right)+\frac{243}{8921600} \sigma_{7}\left(\frac{n}{3}\right)-\frac{91}{209100} \sigma_{7}\left(\frac{n}{4}\right) \\
& -\frac{243}{8921600} \sigma_{7}\left(\frac{n}{6}\right)+\frac{5824}{52275} \sigma_{7}\left(\frac{n}{8}\right)-\frac{243}{69700} \sigma_{7}\left(\frac{n}{12}\right)+\frac{15552}{17425} \sigma_{7}\left(\frac{n}{24}\right)-\frac{44628883}{10465200} a_{1}(n) \\
& -\frac{262223}{2565} a_{1}\left(\frac{n}{2}\right)-\frac{6170373}{129200} a_{1}\left(\frac{n}{3}\right)+\frac{533271424}{654075} a_{1}\left(\frac{n}{4}\right)+\frac{149841}{95} a_{1}\left(\frac{n}{6}\right)+\frac{259052544}{8075} a_{1}\left(\frac{n}{12}\right) \\
& +\frac{826523}{1402200} \Delta_{3,8}(n)-\frac{5443531}{701100} \Delta_{3,8}\left(\frac{n}{2}\right)-\frac{1252291108}{175275} \Delta_{3,8}\left(\frac{n}{4}\right)-\frac{2242527488}{175275} \Delta_{3,8}\left(\frac{n}{8}\right) \\
& +\frac{28267}{7695} a_{4}(n)+\frac{18160}{513} a_{4}\left(\frac{n}{2}\right)+\frac{2825216}{405} a_{4}\left(\frac{n}{4}\right)-2 a_{2}(n)-\frac{1917}{19} a_{2}\left(\frac{n}{3}\right)+6 a_{3}(n) \\
& +\frac{1917}{19} a_{3}\left(\frac{n}{3}\right)+\frac{990}{19} a_{5}(n)-\frac{1440768}{19} a_{5}\left(\frac{n}{2}\right)-19 a_{6}(n)-\frac{45056}{19} a_{6}\left(\frac{n}{2}\right)-\frac{28256}{19} a_{7}(n) \\
& -\frac{45056}{19} a_{8}(n)+\frac{94976}{19} a_{9}(n) \text {, } \\
& N\left(1^{2}, 2^{10}, 3^{2}, 6^{2} ; n\right)=\frac{91}{13382400} \sigma_{7}(n)-\frac{91}{13382400} \sigma_{7}\left(\frac{n}{2}\right)+\frac{243}{4460800} \sigma_{7}\left(\frac{n}{3}\right)-\frac{91}{209100} \sigma_{7}\left(\frac{n}{4}\right) \\
& -\frac{243}{4460800} \sigma_{7}\left(\frac{n}{6}\right)+\frac{5824}{52275} \sigma_{7}\left(\frac{n}{8}\right)-\frac{243}{69700} \sigma_{7}\left(\frac{n}{12}\right)+\frac{15552}{17425} \sigma_{7}\left(\frac{n}{24}\right)+\frac{57512879}{1744200} a_{1}(n) \\
& -\frac{1676}{285} a_{1}\left(\frac{n}{2}\right)-\frac{74494053}{64600} a_{1}\left(\frac{n}{3}\right)+\frac{161038208}{218025} a_{1}\left(\frac{n}{4}\right)-\frac{1748412}{95} a_{1}\left(\frac{n}{6}\right)+\frac{253666944}{8075} a_{1}\left(\frac{n}{12}\right) \\
& +\frac{3327001}{233700} \Delta_{3,8}(n)-\frac{2488277}{116850} \Delta_{3,8}\left(\frac{n}{2}\right)-\frac{1971171016}{58425} \Delta_{3,8}\left(\frac{n}{4}\right)-\frac{1012445696}{58425} \Delta_{3,8}\left(\frac{n}{8}\right) \\
& -\frac{121102}{2565} a_{4}(n)+\frac{12640}{19} a_{4}\left(\frac{n}{2}\right)+\frac{2947072}{135} a_{4}\left(\frac{n}{4}\right)+a_{2}(n)-\frac{48033}{19} a_{2}\left(\frac{n}{3}\right)+3 a_{3}(n) \\
& +\frac{103437}{19} a_{3}\left(\frac{n}{3}\right)-\frac{373788}{19} a_{5}(n)-\frac{4605696}{19} a_{5}\left(\frac{n}{2}\right)+14 a_{6}(n)-\frac{167168}{19} a_{6}\left(\frac{n}{2}\right) \\
& +\frac{126912}{19} a_{7}(n)-\frac{172032}{19} a_{8}(n)-\frac{122880}{19} a_{9}(n) \text {, } \\
& N\left(1^{2}, 2^{10}, 3^{4} ; n\right)=\frac{91}{6691200} \sigma_{7}(n)-\frac{91}{6691200} \sigma_{7}\left(\frac{n}{2}\right)+\frac{243}{2230400} \sigma_{7}\left(\frac{n}{3}\right)-\frac{91}{209100} \sigma_{7}\left(\frac{n}{4}\right) \\
& -\frac{243}{2230400} \sigma_{7}\left(\frac{n}{6}\right)+\frac{5824}{52275} \sigma_{7}\left(\frac{n}{8}\right)-\frac{243}{69700} \sigma_{7}\left(\frac{n}{12}\right)+\frac{15552}{17425} \sigma_{7}\left(\frac{n}{24}\right)+\frac{184648247}{2616300} a_{1}(n) \\
& +\frac{203992}{2565} a_{1}\left(\frac{n}{2}\right)-\frac{73978443}{32300} a_{1}\left(\frac{n}{3}\right)+\frac{685569664}{654075} a_{1}\left(\frac{n}{4}\right)-\frac{3241224}{95} a_{1}\left(\frac{n}{6}\right)+\frac{402701184}{8075} a_{1}\left(\frac{n}{12}\right) \\
& +\frac{4955959}{175275} \Delta_{3,8}(n)+\frac{14601164}{175275} \Delta_{3,8}\left(\frac{n}{2}\right)-\frac{11383414888}{175275} \Delta_{3,8}\left(\frac{n}{4}\right)-\frac{4778413568}{175275} \Delta_{3,8}\left(\frac{n}{8}\right) \\
& -\frac{760712}{7695} a_{4}(n)+\frac{609376}{513} a_{4}\left(\frac{n}{2}\right)+\frac{16910336}{405} a_{4}\left(\frac{n}{4}\right)+6 a_{2}(n)-\frac{91584}{19} a_{2}\left(\frac{n}{3}\right)-2 a_{3}(n) \\
& +\frac{202392}{19} a_{3}\left(\frac{n}{3}\right)-\frac{746856}{19} a_{5}(n)-\frac{8850432}{19} a_{5}\left(\frac{n}{2}\right)+60 a_{6}(n)-\frac{316032}{19} a_{6}\left(\frac{n}{2}\right)+\frac{268096}{19} a_{7}(n) \\
& -\frac{333056}{19} a_{8}(n)-\frac{292096}{19} a_{9}(n) \text {, }
\end{aligned}
$$




$$
\begin{aligned}
& N\left(1^{2}, 2^{12}, 6^{2} ; n\right)=\frac{547}{53529600} \sigma_{7}(n)-\frac{547}{53529600} \sigma_{7}\left(\frac{n}{2}\right)-\frac{729}{17843200} \sigma_{7}\left(\frac{n}{3}\right) \\
& +\frac{547}{418200} \sigma_{7}\left(\frac{n}{4}\right)+\frac{729}{17843200} \sigma_{7}\left(\frac{n}{6}\right)-\frac{17504}{52275} \sigma_{7}\left(\frac{n}{8}\right)-\frac{729}{139400} \sigma_{7}\left(\frac{n}{12}\right) \\
& +\frac{23328}{17425} \sigma_{7}\left(\frac{n}{24}\right)-\frac{6141173}{775200} a_{1}(n)-\frac{13513}{114} a_{1}\left(\frac{n}{2}\right)-\frac{4613301}{258400} a_{1}\left(\frac{n}{3}\right) \\
& +\frac{38599424}{72675} a_{1}\left(\frac{n}{4}\right)-\frac{139131}{38} a_{1}\left(\frac{n}{6}\right)+\frac{103044096}{8075} a_{1}\left(\frac{n}{12}\right) \\
& +\frac{206267}{934800} \Delta_{3,8}(n)-\frac{96400879}{467400} \Delta_{3,8}\left(\frac{n}{2}\right)-\frac{120679514}{58425} \Delta_{3,8}\left(\frac{n}{4}\right) \\
& -\frac{630523264}{58425} \Delta_{3,8}\left(\frac{n}{8}\right)+\frac{4387}{570} a_{4}(n)+\frac{11656}{57} a_{4}\left(\frac{n}{2}\right)+\frac{73216}{45} a_{4}\left(\frac{n}{4}\right) \\
& -\frac{13}{2} a_{2}(n)-\frac{15471}{38} a_{2}\left(\frac{n}{3}\right)+\frac{21}{2} a_{3}(n)+\frac{15471}{38} a_{3}\left(\frac{n}{3}\right)+\frac{9963}{19} a_{5}(n) \\
& -\frac{263808}{19} a_{5}\left(\frac{n}{2}\right)-\frac{107}{2} a_{6}(n)-\frac{16640}{19} a_{6}\left(\frac{n}{2}\right)-\frac{21072}{19} a_{7}(n) \\
& +\frac{384}{19} a_{8}(n)+\frac{50304}{19} a_{9}(n) \text {, } \\
& N\left(1^{2}, 2^{12}, 3^{2} ; n\right)=\frac{547}{26764800} \sigma_{7}(n)-\frac{547}{26764800} \sigma_{7}\left(\frac{n}{2}\right)-\frac{729}{8921600} \sigma_{7}\left(\frac{n}{3}\right) \\
& +\frac{547}{418200} \sigma_{7}\left(\frac{n}{4}\right)+\frac{729}{8921600} \sigma_{7}\left(\frac{n}{6}\right)-\frac{17504}{52275} \sigma_{7}\left(\frac{n}{8}\right)-\frac{729}{139400} \sigma_{7}\left(\frac{n}{12}\right) \\
& +\frac{23328}{17425} \sigma_{7}\left(\frac{n}{24}\right)-\frac{389757457}{3488400} a_{1}(n)-\frac{902119}{2565} a_{1}\left(\frac{n}{2}\right)+\frac{428453199}{129200} a_{1}\left(\frac{n}{3}\right) \\
& +\frac{2559424}{72675} a_{1}\left(\frac{n}{4}\right)+\frac{5226903}{95} a_{1}\left(\frac{n}{6}\right)-\frac{254608704}{8075} a_{1}\left(\frac{n}{12}\right)-\frac{19135483}{467400} \Delta_{3,8}(n) \\
& -\frac{1544509}{233700} \Delta_{3,8}\left(\frac{n}{2}\right)+\frac{4588704716}{58425} \Delta_{3,8}\left(\frac{n}{4}\right)+\frac{624929536}{58425} \Delta_{3,8}\left(\frac{n}{8}\right) \\
& +\frac{391573}{2565} a_{4}(n)-\frac{914032}{513} a_{4}\left(\frac{n}{2}\right)-\frac{1997824}{45} a_{4}\left(\frac{n}{4}\right)-10 a_{2}(n) \\
& +\frac{138348}{19} a_{2}\left(\frac{n}{3}\right)+14 a_{3}(n)-\frac{304560}{19} a_{3}\left(\frac{n}{3}\right)+\frac{1124334}{19} a_{5}(n) \\
& +\frac{9560448}{19} a_{5}\left(\frac{n}{2}\right)-99 a_{6}(n)+\frac{357824}{19} a_{6}\left(\frac{n}{2}\right)-\frac{465504}{19} a_{7}(n) \\
& +\frac{380928}{19} a_{8}(n)+\frac{653568}{19} a_{9}(n) \text {, } \\
& N\left(1^{2}, 2^{14} ; n\right)=\frac{1}{32640} \sigma_{7}(n)-\frac{1}{32640} \sigma_{7}\left(\frac{n}{2}\right)-\frac{1}{255} \sigma_{7}\left(\frac{n}{4}\right)+\frac{256}{255} \sigma_{7}\left(\frac{n}{8}\right) \\
& -\frac{1}{68} a_{1}(n)+2 a_{1}\left(\frac{n}{2}\right)+\frac{1664}{17} a_{1}\left(\frac{n}{4}\right)-a_{4}\left(\frac{n}{4}\right)+5 a_{2}\left(\frac{n}{3}\right),
\end{aligned}
$$




$$
\begin{aligned}
& N\left(1^{3}, 2,3,6^{11} ; n\right)=\frac{1}{10705920} \sigma_{7}(n)-\frac{1}{10705920} \sigma_{7}\left(\frac{n}{2}\right)-\frac{219}{3568640} \sigma_{7}\left(\frac{n}{3}\right)+\frac{1}{167280} \sigma_{7}\left(\frac{n}{4}\right) \\
& +\frac{219}{3568640} \sigma_{7}\left(\frac{n}{6}\right)-\frac{16}{10455} \sigma_{7}\left(\frac{n}{8}\right)-\frac{219}{55760} \sigma_{7}\left(\frac{n}{12}\right)+\frac{3504}{3485} \sigma_{7}\left(\frac{n}{24}\right)-\frac{96943141}{12558240} a_{1}(n) \\
& -\frac{6711791}{46170} a_{1}\left(\frac{n}{2}\right)-\frac{3658377}{51680} a_{1}\left(\frac{n}{3}\right)+\frac{124113184}{130815} a_{1}\left(\frac{n}{4}\right)-\frac{44157}{190} a_{1}\left(\frac{n}{6}\right)+\frac{48638304}{1615} a_{1}\left(\frac{n}{12}\right) \\
& +\frac{91946}{105165} \Delta_{3,8}(n)-\frac{6673567}{46740} \Delta_{3,8}\left(\frac{n}{2}\right)-\frac{695717132}{105165} \Delta_{3,8}\left(\frac{n}{4}\right)-\frac{587681408}{35055} \Delta_{3,8}\left(\frac{n}{8}\right) \\
& +\frac{31604}{4617} a_{4}(n)+\frac{668048}{4617} a_{4}\left(\frac{n}{2}\right)+\frac{513536}{81} a_{4}\left(\frac{n}{4}\right)-\frac{13}{2} a_{2}(n)-\frac{12087}{38} a_{2}\left(\frac{n}{3}\right)+\frac{25}{2} a_{3}(n) \\
& +\frac{11403}{38} a_{3}\left(\frac{n}{3}\right)+\frac{597}{19} a_{5}(n)-\frac{1256576}{19} a_{5}\left(\frac{n}{2}\right)-\frac{99}{2} a_{6}(n)-\frac{43424}{19} a_{6}\left(\frac{n}{2}\right) \\
& -\frac{111680}{57} a_{7}(n)-\frac{37952}{19} a_{8}(n)+\frac{105088}{19} a_{9}(n) \text {, } \\
& N\left(1^{3}, 2,3^{3}, 6^{9} ; n\right)=\frac{1}{5352960} \sigma_{7}(n)-\frac{1}{5352960} \sigma_{7}\left(\frac{n}{2}\right)-\frac{219}{1784320} \sigma_{7}\left(\frac{n}{3}\right)+\frac{1}{167280} \sigma_{7}\left(\frac{n}{4}\right) \\
& +\frac{219}{1784320} \sigma_{7}\left(\frac{n}{6}\right)-\frac{16}{10455} \sigma_{7}\left(\frac{n}{8}\right)-\frac{219}{55760} \sigma_{7}\left(\frac{n}{12}\right)+\frac{3504}{3485} \sigma_{7}\left(\frac{n}{24}\right)-\frac{2791423}{330480} a_{1}(n) \\
& -\frac{169016}{1215} a_{1}\left(\frac{n}{2}\right)-\frac{69051}{1360} a_{1}\left(\frac{n}{3}\right)+\frac{1254368}{1377} a_{1}\left(\frac{n}{4}\right)+\frac{1068}{5} a_{1}\left(\frac{n}{6}\right)+\frac{570528}{17} a_{1}\left(\frac{n}{12}\right) \\
& +\frac{6947}{11070} \Delta_{3,8}(n)-\frac{12035}{82} \Delta_{3,8}\left(\frac{n}{2}\right)-\frac{30228766}{5535} \Delta_{3,8}\left(\frac{n}{4}\right)-\frac{5885824}{369} \Delta_{3,8}\left(\frac{n}{8}\right) \\
& +\frac{1900}{243} a_{4}(n)+\frac{31672}{243} a_{4}\left(\frac{n}{2}\right)+\frac{445952}{81} a_{4}\left(\frac{n}{4}\right)-7 a_{2}(n)-315 a_{2}\left(\frac{n}{3}\right)+13 a_{3}(n) \\
& +261 a_{3}\left(\frac{n}{3}\right)+430 a_{5}(n)-57472 a_{5}\left(\frac{n}{2}\right)-51 a_{6}(n)-1952 a_{6}\left(\frac{n}{2}\right)-\frac{5968}{3} a_{7}(n) \\
& -1600 a_{8}(n)+5312 a_{9}(n) \text {, } \\
& N\left(1^{3}, 2,3^{5}, 6^{7} ; n\right)=\frac{1}{2676480} \sigma_{7}(n)-\frac{1}{2676480} \sigma_{7}\left(\frac{n}{2}\right)-\frac{219}{892160} \sigma_{7}\left(\frac{n}{3}\right)+\frac{1}{167280} \sigma_{7}\left(\frac{n}{4}\right) \\
& +\frac{219}{892160} \sigma_{7}\left(\frac{n}{6}\right)-\frac{16}{10455} \sigma_{7}\left(\frac{n}{8}\right)-\frac{219}{55760} \sigma_{7}\left(\frac{n}{12}\right)+\frac{3504}{3485} \sigma_{7}\left(\frac{n}{24}\right)-\frac{29593187}{3139560} a_{1}(n) \\
& -\frac{2943074}{23085} a_{1}\left(\frac{n}{2}\right)-\frac{332259}{12920} a_{1}\left(\frac{n}{3}\right)+\frac{110419616}{130815} a_{1}\left(\frac{n}{4}\right)+\frac{71322}{95} a_{1}\left(\frac{n}{6}\right)+\frac{62356896}{1615} a_{1}\left(\frac{n}{12}\right) \\
& +\frac{134167}{420660} \Delta_{3,8}(n)-\frac{10959569}{70110} \Delta_{3,8}\left(\frac{n}{2}\right)-\frac{77041448}{21033} \Delta_{3,8}\left(\frac{n}{4}\right)-\frac{509829952}{35055} \Delta_{3,8}\left(\frac{n}{8}\right) \\
& +\frac{42046}{4617} a_{4}(n)+\frac{523552}{4617} a_{4}\left(\frac{n}{2}\right)+\frac{336640}{81} a_{4}\left(\frac{n}{4}\right)-8 a_{2}(n)-\frac{6282}{19} a_{2}\left(\frac{n}{3}\right)+14 a_{3}(n) \\
& +\frac{4572}{19} a_{3}\left(\frac{n}{3}\right)+\frac{18076}{19} a_{5}(n)-\frac{825600}{19} a_{5}\left(\frac{n}{2}\right)-54 a_{6}(n)-\frac{26976}{19} a_{6}\left(\frac{n}{2}\right) \\
& -\frac{112768}{57} a_{7}(n)-\frac{17856}{19} a_{8}(n)+\frac{91648}{19} a_{9}(n) \text {, }
\end{aligned}
$$




$$
\begin{aligned}
& N\left(1^{3}, 2,3^{7}, 6^{5} ; n\right)=\frac{1}{1338240} \sigma_{7}(n)-\frac{1}{1338240} \sigma_{7}\left(\frac{n}{2}\right)-\frac{219}{446080} \sigma_{7}\left(\frac{n}{3}\right)+\frac{1}{167280} \sigma_{7}\left(\frac{n}{4}\right) \\
& +\frac{219}{446080} \sigma_{7}\left(\frac{n}{6}\right)-\frac{16}{10455} \sigma_{7}\left(\frac{n}{8}\right)-\frac{219}{55760} \sigma_{7}\left(\frac{n}{12}\right)+\frac{3504}{3485} \sigma_{7}\left(\frac{n}{24}\right)-\frac{285556}{26163} a_{1}(n) \\
& -\frac{88802}{855} a_{1}\left(\frac{n}{2}\right)+\frac{6039}{646} a_{1}\left(\frac{n}{3}\right)+\frac{10432544}{14535} a_{1}\left(\frac{n}{4}\right)+\frac{151542}{95} a_{1}\left(\frac{n}{6}\right)+\frac{79394976}{1615} a_{1}\left(\frac{n}{12}\right) \\
& -\frac{3155}{28044} \Delta_{3,8}(n)-\frac{12370771}{70110} \Delta_{3,8}\left(\frac{n}{2}\right)-\frac{403148}{779} \Delta_{3,8}\left(\frac{n}{4}\right)-\frac{46417088}{3895} \Delta_{3,8}\left(\frac{n}{8}\right) \\
& +\frac{16970}{1539} a_{4}(n)+\frac{15280}{171} a_{4}\left(\frac{n}{2}\right)+\frac{15616}{9} a_{4}\left(\frac{n}{4}\right)-10 a_{2}(n)-\frac{7416}{19} a_{2}\left(\frac{n}{3}\right)+16 a_{3}(n) \\
& +\frac{5022}{19} a_{3}\left(\frac{n}{3}\right)+\frac{32560}{19} a_{5}(n)-\frac{352000}{19} a_{5}\left(\frac{n}{2}\right)-60 a_{6}(n)-\frac{8928}{19} a_{6}\left(\frac{n}{2}\right)-\frac{35616}{19} a_{7}(n) \\
& +\frac{5056}{19} a_{8}(n)+\frac{71360}{19} a_{9}(n) \text {, } \\
& N\left(1^{3}, 2,3^{9}, 6^{3} ; n\right)=\frac{1}{669120} \sigma_{7}(n)-\frac{1}{669120} \sigma_{7}\left(\frac{n}{2}\right)-\frac{219}{223040} \sigma_{7}\left(\frac{n}{3}\right)+\frac{1}{167280} \sigma_{7}\left(\frac{n}{4}\right) \\
& +\frac{219}{223040} \sigma_{7}\left(\frac{n}{6}\right)-\frac{16}{10455} \sigma_{7}\left(\frac{n}{8}\right)-\frac{219}{55760} \sigma_{7}\left(\frac{n}{12}\right)+\frac{3504}{3485} \sigma_{7}\left(\frac{n}{24}\right)-\frac{5260792}{392445} a_{1}(n) \\
& -\frac{251200}{4617} a_{1}\left(\frac{n}{2}\right)+\frac{104451}{1615} a_{1}\left(\frac{n}{3}\right)+\frac{20123872}{43605} a_{1}\left(\frac{n}{4}\right)+\frac{59628}{19} a_{1}\left(\frac{n}{6}\right)+\frac{114793056}{1615} a_{1}\left(\frac{n}{12}\right) \\
& -\frac{166717}{210330} \Delta_{3,8}(n)-\frac{2536699}{11685} \Delta_{3,8}\left(\frac{n}{2}\right)+\frac{570958844}{105165} \Delta_{3,8}\left(\frac{n}{4}\right)-\frac{25923648}{3895} \Delta_{3,8}\left(\frac{n}{8}\right) \\
& +\frac{65548}{4617} a_{4}(n)+\frac{226000}{4617} a_{4}\left(\frac{n}{2}\right)-\frac{78592}{27} a_{4}\left(\frac{n}{4}\right)-14 a_{2}(n)-\frac{10170}{19} a_{2}\left(\frac{n}{3}\right)+20 a_{3}(n) \\
& +\frac{7092}{19} a_{3}\left(\frac{n}{3}\right)+\frac{56368}{19} a_{5}(n)+\frac{550976}{19} a_{5}\left(\frac{n}{2}\right)-72 a_{6}(n)+\frac{25696}{19} a_{6}\left(\frac{n}{2}\right)-\frac{89344}{57} a_{7}(n) \\
& +\frac{49408}{19} a_{8}(n)+\frac{27648}{19} a_{9}(n) \text {, } \\
& N\left(1^{3}, 2,3^{11}, 6 ; n\right)=\frac{1}{334560} \sigma_{7}(n)-\frac{1}{334560} \sigma_{7}\left(\frac{n}{2}\right)-\frac{219}{111520} \sigma_{7}\left(\frac{n}{3}\right)+\frac{1}{167280} \sigma_{7}\left(\frac{n}{4}\right) \\
& +\frac{219}{111520} \sigma_{7}\left(\frac{n}{6}\right)-\frac{16}{10455} \sigma_{7}\left(\frac{n}{8}\right)-\frac{219}{55760} \sigma_{7}\left(\frac{n}{12}\right)+\frac{3504}{3485} \sigma_{7}\left(\frac{n}{24}\right)-\frac{6950003}{392445} a_{1}(n) \\
& +\frac{232924}{4617} a_{1}\left(\frac{n}{2}\right)+\frac{263829}{1615} a_{1}\left(\frac{n}{3}\right)-\frac{3494432}{43605} a_{1}\left(\frac{n}{4}\right)+\frac{117744}{19} a_{1}\left(\frac{n}{6}\right)+\frac{187586784}{1615} a_{1}\left(\frac{n}{12}\right) \\
& -\frac{210874}{105165} \Delta_{3,8}(n)-\frac{10459438}{35055} \Delta_{3,8}\left(\frac{n}{2}\right)+\frac{1796353396}{105165} \Delta_{3,8}\left(\frac{n}{4}\right)+\frac{16805568}{3895} \Delta_{3,8}\left(\frac{n}{8}\right) \\
& +\frac{91016}{4617} a_{4}(n)-\frac{115600}{4617} a_{4}\left(\frac{n}{2}\right)-\frac{327424}{27} a_{4}\left(\frac{n}{4}\right)-22 a_{2}(n)-\frac{16002}{19} a_{2}\left(\frac{n}{3}\right)+28 a_{3}(n) \\
& +\frac{12240}{19} a_{3}\left(\frac{n}{3}\right)+\frac{97200}{19} a_{5}(n)+\frac{2333120}{19} a_{5}\left(\frac{n}{2}\right)-96 a_{6}(n)+\frac{94368}{19} a_{6}\left(\frac{n}{2}\right) \\
& -\frac{44480}{57} a_{7}(n)+\frac{136320}{19} a_{8}(n)-\frac{65920}{19} a_{9}(n) \text {, }
\end{aligned}
$$




$$
\begin{aligned}
& N\left(1^{3}, 2^{3}, 3,6^{9} ; n\right)=\frac{13}{53529600} \sigma_{7}(n)-\frac{13}{53529600} \sigma_{7}\left(\frac{n}{2}\right)+\frac{1089}{17843200} \sigma_{7}\left(\frac{n}{3}\right)-\frac{13}{836400} \sigma_{7}\left(\frac{n}{4}\right) \\
& -\frac{1089}{17843200} \sigma_{7}\left(\frac{n}{6}\right)+\frac{208}{52275} \sigma_{7}\left(\frac{n}{8}\right)-\frac{1089}{278800} \sigma_{7}\left(\frac{n}{12}\right)+\frac{17424}{17425} \sigma_{7}\left(\frac{n}{24}\right)-\frac{128355679}{20930400} a_{1}(n) \\
& -\frac{2553209}{15390} a_{1}\left(\frac{n}{2}\right)-\frac{28175049}{258400} a_{1}\left(\frac{n}{3}\right)+\frac{696635488}{654075} a_{1}\left(\frac{n}{4}\right)-\frac{212409}{190} a_{1}\left(\frac{n}{6}\right)+\frac{182010528}{8075} a_{1}\left(\frac{n}{12}\right) \\
& +\frac{1887037}{1402200} \Delta_{3,8}(n)-\frac{21059221}{175275} \Delta_{3,8}\left(\frac{n}{2}\right)-\frac{1704961316}{175275} \Delta_{3,8}\left(\frac{n}{4}\right)-\frac{3369501056}{175275} \Delta_{3,8}\left(\frac{n}{8}\right) \\
& +\frac{36833}{7695} a_{4}(n)+\frac{260144}{1539} a_{4}\left(\frac{n}{2}\right)+\frac{3533312}{405} a_{4}\left(\frac{n}{4}\right)-\frac{9}{2} a_{2}(n)-\frac{11367}{38} a_{2}\left(\frac{n}{3}\right)+\frac{21}{2} a_{3}(n) \\
& +\frac{13419}{38} a_{3}\left(\frac{n}{3}\right)-\frac{14547}{19} a_{5}(n)-\frac{1730304}{19} a_{5}\left(\frac{n}{2}\right)-\frac{87}{2} a_{6}(n)-\frac{61024}{19} a_{6}\left(\frac{n}{2}\right) \\
& -\frac{37184}{19} a_{7}(n)-\frac{59200}{19} a_{8}(n)+\frac{120832}{19} a_{9}(n), \\
& N\left(1^{3}, 2^{3}, 3^{3}, 6^{7} ; n\right)=\frac{13}{26764800} \sigma_{7}(n)-\frac{13}{26764800} \sigma_{7}\left(\frac{n}{2}\right)+\frac{1089}{8921600} \sigma_{7}\left(\frac{n}{3}\right)-\frac{13}{836400} \sigma_{7}\left(\frac{n}{4}\right) \\
& -\frac{1089}{8921600} \sigma_{7}\left(\frac{n}{6}\right)+\frac{208}{52275} \sigma_{7}\left(\frac{n}{8}\right)-\frac{1089}{278800} \sigma_{7}\left(\frac{n}{12}\right)+\frac{17424}{17425} \sigma_{7}\left(\frac{n}{24}\right)-\frac{43225799}{10465200} a_{1}(n) \\
& -\frac{276140}{1539} a_{1}\left(\frac{n}{2}\right)-\frac{21081969}{129200} a_{1}\left(\frac{n}{3}\right)+\frac{751927648}{654075} a_{1}\left(\frac{n}{4}\right)-\frac{43200}{19} a_{1}\left(\frac{n}{6}\right)+\frac{117677088}{8075} a_{1}\left(\frac{n}{12}\right) \\
& +\frac{1411847}{701100} \Delta_{3,8}(n)-\frac{18989932}{175275} \Delta_{3,8}\left(\frac{n}{2}\right)-\frac{2200979726}{175275} \Delta_{3,8}\left(\frac{n}{4}\right)-\frac{3695480576}{175275} \Delta_{3,8}\left(\frac{n}{8}\right) \\
& +\frac{16286}{7695} a_{4}(n)+\frac{313112}{1539} a_{4}\left(\frac{n}{2}\right)+\frac{4355072}{405} a_{4}\left(\frac{n}{4}\right)-3 a_{2}(n)-\frac{6237}{19} a_{2}\left(\frac{n}{3}\right)+9 a_{3}(n) \\
& +\frac{9315}{19} a_{3}\left(\frac{n}{3}\right)-\frac{34566}{19} a_{5}(n)-\frac{2132736}{19} a_{5}\left(\frac{n}{2}\right)-39 a_{6}(n)-\frac{76448}{19} a_{6}\left(\frac{n}{2}\right)-\frac{33936}{19} a_{7}(n) \\
& -\frac{78272}{19} a_{8}(n)+\frac{128576}{19} a_{9}(n) \text {, } \\
& N\left(1^{3}, 2^{3}, 3^{5}, 6^{5} ; n\right)=\frac{13}{13382400} \sigma_{7}(n)-\frac{13}{13382400} \sigma_{7}\left(\frac{n}{2}\right)+\frac{1089}{4460800} \sigma_{7}\left(\frac{n}{3}\right)-\frac{13}{836400} \sigma_{7}\left(\frac{n}{4}\right) \\
& -\frac{1089}{4460800} \sigma_{7}\left(\frac{n}{6}\right)+\frac{208}{52275} \sigma_{7}\left(\frac{n}{8}\right)-\frac{1089}{278800} \sigma_{7}\left(\frac{n}{12}\right)+\frac{17424}{17425} \sigma_{7}\left(\frac{n}{24}\right)-\frac{7213169}{5232600} a_{1}(n) \\
& -\frac{1633118}{7695} a_{1}\left(\frac{n}{2}\right)-\frac{15036939}{64600} a_{1}\left(\frac{n}{3}\right)+\frac{875361248}{654075} a_{1}\left(\frac{n}{4}\right)-\frac{361638}{95} a_{1}\left(\frac{n}{6}\right)-\frac{5457312}{8075} a_{1}\left(\frac{n}{12}\right) \\
& +\frac{2013739}{701100} \Delta_{3,8}(n)-\frac{27677923}{350550} \Delta_{3,8}\left(\frac{n}{2}\right)-\frac{3079785616}{175275} \Delta_{3,8}\left(\frac{n}{4}\right)-\frac{4391037376}{175275} \Delta_{3,8}\left(\frac{n}{8}\right) \\
& -\frac{11498}{7695} a_{4}(n)+\frac{381376}{1539} a_{4}\left(\frac{n}{2}\right)+\frac{5892352}{405} a_{4}\left(\frac{n}{4}\right)-\frac{5562}{19} a_{2}\left(\frac{n}{3}\right)+6 a_{3}(n)+\frac{10692}{19} a_{3}\left(\frac{n}{3}\right) \\
& -\frac{61764}{19} a_{5}(n)+-\frac{2879616}{19} a_{5}\left(\frac{n}{2}\right)-30 a_{6}(n)-\frac{104736}{19} a_{6}\left(\frac{n}{2}\right)-\frac{33856}{19} a_{7}(n) \\
& -\frac{113856}{19} a_{8}(n)+\frac{154752}{19} a_{9}(n) \text {, }
\end{aligned}
$$




$$
\begin{aligned}
& N\left(1^{3}, 2^{3}, 3^{7}, 6^{3} ; n\right)=\frac{13}{6691200} \sigma_{7}(n)-\frac{13}{6691200} \sigma_{7}\left(\frac{n}{2}\right)+\frac{1089}{2230400} \sigma_{7}\left(\frac{n}{3}\right)-\frac{13}{836400} \sigma_{7}\left(\frac{n}{4}\right) \\
& -\frac{1089}{2230400} \sigma_{7}\left(\frac{n}{6}\right)+\frac{208}{52275} \sigma_{7}\left(\frac{n}{8}\right)-\frac{1089}{278800} \sigma_{7}\left(\frac{n}{12}\right)+\frac{17424}{17425} \sigma_{7}\left(\frac{n}{24}\right)+\frac{647588}{218025} a_{1}(n) \\
& -\frac{719414}{2565} a_{1}\left(\frac{n}{2}\right)-\frac{5359257}{16150} a_{1}\left(\frac{n}{3}\right)+\frac{371838496}{218025} a_{1}\left(\frac{n}{4}\right)-\frac{594162}{95} a_{1}\left(\frac{n}{6}\right)-\frac{261811872}{8075} a_{1}\left(\frac{n}{12}\right) \\
& +\frac{318917}{77900} \Delta_{3,8}(n)-\frac{687559}{38950} \Delta_{3,8}\left(\frac{n}{2}\right)-\frac{518717524}{19475} \Delta_{3,8}\left(\frac{n}{4}\right)-\frac{1909295552}{58425} \Delta_{3,8}\left(\frac{n}{8}\right) \\
& -\frac{18122}{2565} a_{4}(n)+\frac{161008}{513} a_{4}\left(\frac{n}{2}\right)+\frac{2910464}{135} a_{4}\left(\frac{n}{4}\right)+6 a_{2}(n)-\frac{2376}{19} a_{2}\left(\frac{n}{3}\right)+\frac{9558}{19} a_{3}\left(\frac{n}{3}\right) \\
& -\frac{103152}{19} a_{5}(n)-\frac{4248192}{19} a_{5}\left(\frac{n}{2}\right)-12 a_{6}(n)-\frac{156832}{19} a_{6}\left(\frac{n}{2}\right)-\frac{39072}{19} a_{7}(n) \\
& -\frac{180544}{19} a_{8}(n)+\frac{213952}{19} a_{9}(n) \\
& N\left(1^{3}, 2^{3}, 3^{9}, 6 ; n\right)=\frac{13}{3345600} \sigma_{7}(n)-\frac{13}{3345600} \sigma_{7}\left(\frac{n}{2}\right)+\frac{1089}{1115200} \sigma_{7}\left(\frac{n}{3}\right)-\frac{13}{836400} \sigma_{7}\left(\frac{n}{4}\right) \\
& -\frac{1089}{1115200} \sigma_{7}\left(\frac{n}{6}\right)+\frac{208}{52275} \sigma_{7}\left(\frac{n}{8}\right)-\frac{1089}{278800} \sigma_{7}\left(\frac{n}{12}\right)+\frac{17424}{17425} \sigma_{7}\left(\frac{n}{24}\right)+\frac{2260436}{218025} a_{1}(n) \\
& -\frac{368648}{855} a_{1}\left(\frac{n}{2}\right)-\frac{3973077}{8075} a_{1}\left(\frac{n}{3}\right)+\frac{539444896}{218025} a_{1}\left(\frac{n}{4}\right)-\frac{1036692}{95} a_{1}\left(\frac{n}{6}\right)-\frac{792783072}{8075} a_{1}\left(\frac{n}{12}\right) \\
& +\frac{709111}{116850} \Delta_{3,8}(n)+\frac{6213173}{58425} \Delta_{3,8}\left(\frac{n}{2}\right)-\frac{2596256972}{58425} \Delta_{3,8}\left(\frac{n}{4}\right)-\frac{2831500352}{58425} \Delta_{3,8}\left(\frac{n}{8}\right) \\
& -\frac{42164}{2565} a_{4}(n)+\frac{74128}{171} a_{4}\left(\frac{n}{2}\right)+\frac{4792064}{135} a_{4}\left(\frac{n}{4}\right)+18 a_{2}(n)+\frac{5886}{19} a_{2}\left(\frac{n}{3}\right)-12 a_{3}(n) \\
& +\frac{3348}{19} a_{3}\left(\frac{n}{3}\right)-\frac{172752}{19} a_{5}(n)-\frac{6957120}{19} a_{5}\left(\frac{n}{2}\right)+24 a_{6}(n)-\frac{260704}{19} a_{6}\left(\frac{n}{2}\right)-\frac{56576}{19} a_{7}(n) \\
& -\frac{313600}{19} a_{8}(n)+\frac{345088}{19} a_{9}(n) \text {, } \\
& N\left(1^{3}, 2^{5}, 3,6^{7} ; n\right)=\frac{1}{1305600} \sigma_{7}(n)-\frac{1}{1305600} \sigma_{7}\left(\frac{n}{2}\right)-\frac{27}{435200} \sigma_{7}\left(\frac{n}{3}\right)+\frac{1}{20400} \sigma_{7}\left(\frac{n}{4}\right) \\
& +\frac{27}{435200} \sigma_{7}\left(\frac{n}{6}\right)-\frac{16}{1275} \sigma_{7}\left(\frac{n}{8}\right)-\frac{27}{6800} \sigma_{7}\left(\frac{n}{12}\right)+\frac{432}{425} \sigma_{7}\left(\frac{n}{24}\right)-\frac{78081661}{6976800} a_{1}(n) \\
& -\frac{657283}{5130} a_{1}\left(\frac{n}{2}\right)+\frac{4711527}{258400} a_{1}\left(\frac{n}{3}\right)+\frac{19483232}{24225} a_{1}\left(\frac{n}{4}\right)+\frac{205551}{190} a_{1}\left(\frac{n}{6}\right)+\frac{328735584}{8075} a_{1}\left(\frac{n}{12}\right) \\
& -\frac{427}{1900} \Delta_{3,8}(n)-\frac{980561}{5700} \Delta_{3,8}\left(\frac{n}{2}\right)-\frac{3023636}{1425} \Delta_{3,8}\left(\frac{n}{4}\right)-\frac{6470272}{475} \Delta_{3,8}\left(\frac{n}{8}\right) \\
& +\frac{29282}{2565} a_{4}(n)+\frac{54256}{513} a_{4}\left(\frac{n}{2}\right)+\frac{45568}{15} a_{4}\left(\frac{n}{4}\right)-\frac{17}{2} a_{2}(n)-\frac{11259}{38} a_{2}\left(\frac{n}{3}\right)+\frac{29}{2} a_{3}(n) \\
& +\frac{5103}{38} a_{3}\left(\frac{n}{3}\right)+\frac{33273}{19} a_{5}(n)-\frac{597888}{19} a_{5}\left(\frac{n}{2}\right)-\frac{119}{2} a_{6}(n)-\frac{18976}{19} a_{6}\left(\frac{n}{2}\right)-\frac{40704}{19} a_{7}(n) \\
& -\frac{6208}{19} a_{8}(n)+\frac{90240}{19} a_{9}(n)
\end{aligned}
$$




$$
\begin{aligned}
& N\left(1^{3}, 2^{5}, 3^{3}, 6^{5} ; n\right)=\frac{1}{652800} \sigma_{7}(n)-\frac{1}{652800} \sigma_{7}\left(\frac{n}{2}\right)-\frac{27}{217600} \sigma_{7}\left(\frac{n}{3}\right)+\frac{1}{20400} \sigma_{7}\left(\frac{n}{4}\right) \\
& +\frac{27}{217600} \sigma_{7}\left(\frac{n}{6}\right)-\frac{16}{1275} \sigma_{7}\left(\frac{n}{8}\right)-\frac{27}{6800} \sigma_{7}\left(\frac{n}{12}\right)+\frac{432}{425} \sigma_{7}\left(\frac{n}{24}\right)-\frac{57762581}{3488400} a_{1}(n) \\
& -\frac{98488}{855} a_{1}\left(\frac{n}{2}\right)+\frac{21033567}{129200} a_{1}\left(\frac{n}{3}\right)+\frac{146865248}{218025} a_{1}\left(\frac{n}{4}\right)+\frac{361908}{95} a_{1}\left(\frac{n}{6}\right)+\frac{410400864}{8075} a_{1}\left(\frac{n}{12}\right) \\
& -\frac{2863}{1425} \Delta_{3,8}(n)-\frac{569141}{2850} \Delta_{3,8}\left(\frac{n}{2}\right)+\frac{5379094}{1425} \Delta_{3,8}\left(\frac{n}{4}\right)-\frac{14999936}{1425} \Delta_{3,8}\left(\frac{n}{8}\right) \\
& +\frac{47624}{2565} a_{4}(n)+\frac{4568}{171} a_{4}\left(\frac{n}{2}\right)-\frac{132608}{135} a_{4}\left(\frac{n}{4}\right)-11 a_{2}(n)-\frac{2349}{19} a_{2}\left(\frac{n}{3}\right)+17 a_{3}(n) \\
& -\frac{6885}{19} a_{3}\left(\frac{n}{3}\right)+\frac{84690}{19} a_{5}(n)+\frac{217728}{19} a_{5}\left(\frac{n}{2}\right)-71 a_{6}(n)+\frac{11936}{19} a_{6}\left(\frac{n}{2}\right)-\frac{53904}{19} a_{7}(n) \\
& +\frac{30784}{19} a_{8}(n)+\frac{90432}{19} a_{9}(n) \text {, } \\
& N\left(1^{3}, 2^{5}, 3^{5}, 6^{3} ; n\right)=\frac{1}{326400} \sigma_{7}(n)-\frac{1}{326400} \sigma_{7}\left(\frac{n}{2}\right)-\frac{27}{108800} \sigma_{7}\left(\frac{n}{3}\right)+\frac{1}{20400} \sigma_{7}\left(\frac{n}{4}\right) \\
& +\frac{27}{108800} \sigma_{7}\left(\frac{n}{6}\right)-\frac{16}{1275} \sigma_{7}\left(\frac{n}{8}\right)-\frac{27}{6800} \sigma_{7}\left(\frac{n}{12}\right)+\frac{432}{425} \sigma_{7}\left(\frac{n}{24}\right)-\frac{40716851}{1744200} a_{1}(n) \\
& -\frac{185906}{2565} a_{1}\left(\frac{n}{2}\right)+\frac{21313557}{64600} a_{1}\left(\frac{n}{3}\right)+\frac{82270688}{218025} a_{1}\left(\frac{n}{4}\right)+\frac{664362}{95} a_{1}\left(\frac{n}{6}\right)+\frac{589447584}{8075} a_{1}\left(\frac{n}{12}\right) \\
& -\frac{23209}{5700} \Delta_{3,8}(n)-\frac{779027}{2850} \Delta_{3,8}\left(\frac{n}{2}\right)+\frac{18932504}{1425} \Delta_{3,8}\left(\frac{n}{4}\right)-\frac{5742016}{1425} \Delta_{3,8}\left(\frac{n}{8}\right) \\
& +\frac{70318}{2565} a_{4}(n)-\frac{27104}{513} a_{4}\left(\frac{n}{2}\right)-\frac{1081088}{135} a_{4}\left(\frac{n}{4}\right)-16 a_{2}(n)-\frac{1458}{19} a_{2}\left(\frac{n}{3}\right)+22 a_{3}(n) \\
& -\frac{13932}{19} a_{3}\left(\frac{n}{3}\right)+\frac{145260}{19} a_{5}(n)+\frac{1622016}{19} a_{5}\left(\frac{n}{2}\right)-94 a_{6}(n)+\frac{64288}{19} a_{6}\left(\frac{n}{2}\right) \\
& -\frac{61824}{19} a_{7}(n)+\frac{95296}{19} a_{8}(n)+\frac{59904}{19} a_{9}(n) \text {, } \\
& N\left(1^{3}, 2^{5}, 3^{7}, 6 ; n\right)=\frac{1}{163200} \sigma_{7}(n)-\frac{1}{163200} \sigma_{7}\left(\frac{n}{2}\right)-\frac{27}{54400} \sigma_{7}\left(\frac{n}{3}\right)+\frac{1}{20400} \sigma_{7}\left(\frac{n}{4}\right) \\
& +\frac{27}{54400} \sigma_{7}\left(\frac{n}{6}\right)-\frac{16}{1275} \sigma_{7}\left(\frac{n}{8}\right)-\frac{27}{6800} \sigma_{7}\left(\frac{n}{12}\right)+\frac{432}{425} \sigma_{7}\left(\frac{n}{24}\right)-\frac{7322044}{218025} a_{1}(n) \\
& +\frac{28418}{2565} a_{1}\left(\frac{n}{2}\right)+\frac{8608491}{16150} a_{1}\left(\frac{n}{3}\right)+-\frac{8440544}{72675} a_{1}\left(\frac{n}{4}\right)+\frac{1072494}{95} a_{1}\left(\frac{n}{6}\right)+\frac{966635424}{8075} a_{1}\left(\frac{n}{12}\right) \\
& -\frac{37493}{5700} \Delta_{3,8}(n)-\frac{406043}{950} \Delta_{3,8}\left(\frac{n}{2}\right)+\frac{40795564}{1425} \Delta_{3,8}\left(\frac{n}{4}\right)+\frac{9650624}{1425} \Delta_{3,8}\left(\frac{n}{8}\right) \\
& +\frac{103006}{2565} a_{4}(n)-\frac{70192}{513} a_{4}\left(\frac{n}{2}\right)-\frac{883456}{45} a_{4}\left(\frac{n}{4}\right)-26 a_{2}(n)-\frac{5184}{19} a_{2}\left(\frac{n}{3}\right)+32 a_{3}(n) \\
& -\frac{16362}{19} a_{3}\left(\frac{n}{3}\right)+\frac{227376}{19} a_{5}(n)+\frac{3923712}{19} a_{5}\left(\frac{n}{2}\right)-140 a_{6}(n)+\frac{150688}{19} a_{6}\left(\frac{n}{2}\right) \\
& -\frac{65184}{19} a_{7}(n)+\frac{206016}{19} a_{8}(n)-\frac{14400}{19} a_{9}(n) \text {, }
\end{aligned}
$$




$$
\begin{aligned}
& N\left(1^{3}, 2^{7}, 3,6^{5} ; n\right)=\frac{121}{53529600} \sigma_{7}(n)-\frac{121}{53529600} \sigma_{7}\left(\frac{n}{2}\right)+\frac{1053}{17843200} \sigma_{7}\left(\frac{n}{3}\right)-\frac{121}{836400} \sigma_{7}\left(\frac{n}{4}\right) \\
& -\frac{1053}{17843200} \sigma_{7}\left(\frac{n}{6}\right)+\frac{1936}{52275} \sigma_{7}\left(\frac{n}{8}\right)-\frac{1053}{278800} \sigma_{7}\left(\frac{n}{12}\right)+\frac{16848}{17425} \sigma_{7}\left(\frac{n}{24}\right)+\frac{906517}{20930400} a_{1}(n) \\
& -\frac{829639}{5130} a_{1}\left(\frac{n}{2}\right)-\frac{68516973}{258400} a_{1}\left(\frac{n}{3}\right)+\frac{731344096}{654075} a_{1}\left(\frac{n}{4}\right)-\frac{742437}{190} a_{1}\left(\frac{n}{6}\right)+\frac{149810976}{8075} a_{1}\left(\frac{n}{12}\right) \\
& +\frac{4589699}{1402200} \Delta_{3,8}(n)-\frac{22542629}{350550} \Delta_{3,8}\left(\frac{n}{2}\right)-\frac{2666701132}{175275} \Delta_{3,8}\left(\frac{n}{4}\right)-\frac{3682631552}{175275} \Delta_{3,8}\left(\frac{n}{8}\right) \\
& -\frac{25529}{7695} a_{4}(n)+\frac{116848}{513} a_{4}\left(\frac{n}{2}\right)+\frac{5026304}{405} a_{4}\left(\frac{n}{4}\right)-\frac{5}{2} a_{2}(n)-\frac{21411}{38} a_{2}\left(\frac{n}{3}\right)+\frac{17}{2} a_{3}(n) \\
& +\frac{39879}{38} a_{3}\left(\frac{n}{3}\right)-\frac{65511}{19} a_{5}(n)-\frac{2500608}{19} a_{5}\left(\frac{n}{2}\right)-\frac{51}{2} a_{6}(n)-\frac{88800}{19} a_{6}\left(\frac{n}{2}\right)-\frac{16256}{19} a_{7}(n) \\
& -\frac{89408}{19} a_{8}(n)+\frac{103424}{19} a_{9}(n) \text {, } \\
& N\left(1^{3}, 2^{7}, 3^{3}, 6^{3} ; n\right)=\frac{121}{26764800} \sigma_{7}(n)-\frac{121}{26764800} \sigma_{7}\left(\frac{n}{2}\right)+\frac{1053}{8921600} \sigma_{7}\left(\frac{n}{3}\right)-\frac{121}{836400} \sigma_{7}\left(\frac{n}{4}\right) \\
& -\frac{1053}{8921600} \sigma_{7}\left(\frac{n}{6}\right)+\frac{1936}{52275} \sigma_{7}\left(\frac{n}{8}\right)-\frac{1053}{278800} \sigma_{7}\left(\frac{n}{12}\right)+\frac{16848}{17425} \sigma_{7}\left(\frac{n}{24}\right)+\frac{145001917}{10465200} a_{1}(n) \\
& -\frac{43988}{285} a_{1}\left(\frac{n}{2}\right)-\frac{84765573}{129200} a_{1}\left(\frac{n}{3}\right)+\frac{843516896}{654075} a_{1}\left(\frac{n}{4}\right)-\frac{1006416}{95} a_{1}\left(\frac{n}{6}\right)+\frac{99382176}{8075} a_{1}\left(\frac{n}{12}\right) \\
& +\frac{5678249}{701100} \Delta_{3,8}(n)-\frac{3457949}{175275} \Delta_{3,8}\left(\frac{n}{2}\right)-\frac{4832954582}{175275} \Delta_{3,8}\left(\frac{n}{4}\right)-\frac{4510634752}{175275} \Delta_{3,8}\left(\frac{n}{8}\right) \\
& -\frac{168958}{7695} a_{4}(n)+\frac{24488}{57} a_{4}\left(\frac{n}{2}\right)+\frac{8233984}{405} a_{4}\left(\frac{n}{4}\right)+a_{2}(n)-\frac{23841}{19} a_{2}\left(\frac{n}{3}\right)+5 a_{3}(n) \\
& +\frac{51543}{19} a_{3}\left(\frac{n}{3}\right)-\frac{200142}{19} a_{5}(n)-\frac{4151040}{19} a_{5}\left(\frac{n}{2}\right)-3 a_{6}(n)-\frac{150304}{19} a_{6}\left(\frac{n}{2}\right) \\
& +\frac{30128}{19} a_{7}(n)-\frac{159424}{19} a_{8}(n)+\frac{62272}{19} a_{9}(n) \text {, } \\
& N\left(1^{3}, 2^{7}, 3^{5}, 6 ; n\right)=\frac{121}{13382400} \sigma_{7}(n)-\frac{121}{13382400} \sigma_{7}\left(\frac{n}{2}\right)+\frac{1053}{4460800} \sigma_{7}\left(\frac{n}{3}\right)-\frac{121}{836400} \sigma_{7}\left(\frac{n}{4}\right) \\
& -\frac{1053}{4460800} \sigma_{7}\left(\frac{n}{6}\right)+\frac{1936}{52275} \sigma_{7}\left(\frac{n}{8}\right)-\frac{1053}{278800} \sigma_{7}\left(\frac{n}{12}\right)+\frac{16848}{17425} \sigma_{7}\left(\frac{n}{24}\right)+\frac{158241347}{5232600} a_{1}(n) \\
& -\frac{429722}{2565} a_{1}\left(\frac{n}{2}\right)-\frac{69234543}{64600} a_{1}\left(\frac{n}{3}\right)+\frac{1048594016}{654075} a_{1}\left(\frac{n}{4}\right)-\frac{1606086}{95} a_{1}\left(\frac{n}{6}\right)-\frac{59395104}{8075} a_{1}\left(\frac{n}{12}\right) \\
& +\frac{9275543}{701100} \Delta_{3,8}(n)+\frac{42438449}{350550} \Delta_{3,8}\left(\frac{n}{2}\right)-\frac{7734429872}{175275} \Delta_{3,8}\left(\frac{n}{4}\right)-\frac{5898035392}{175275} \Delta_{3,8}\left(\frac{n}{8}\right) \\
& -\frac{334546}{7695} a_{4}(n)+\frac{302272}{513} a_{4}\left(\frac{n}{2}\right)+\frac{12930304}{405} a_{4}\left(\frac{n}{4}\right)+8 a_{2}(n)-\frac{32994}{19} a_{2}\left(\frac{n}{3}\right)-2 a_{3}(n) \\
& +\frac{79164}{19} a_{3}\left(\frac{n}{3}\right)-\frac{343188}{19} a_{5}(n)-\frac{6534528}{19} a_{5}\left(\frac{n}{2}\right)+42 a_{6}(n)-\frac{236192}{19} a_{6}\left(\frac{n}{2}\right) \\
& +\frac{71360}{19} a_{7}(n)-\frac{262336}{19} a_{8}(n)+\frac{52864}{19} a_{9}(n),
\end{aligned}
$$




$$
\begin{aligned}
& N\left(1^{3}, 2^{9}, 3,6^{3} ; n\right)=\frac{73}{10705920} \sigma_{7}(n)-\frac{73}{10705920} \sigma_{7}\left(\frac{n}{2}\right)-\frac{243}{3568640} \sigma_{7}\left(\frac{n}{3}\right)+\frac{73}{167280} \sigma_{7}\left(\frac{n}{4}\right) \\
& +\frac{243}{3568640} \sigma_{7}\left(\frac{n}{6}\right)-\frac{1168}{10455} \sigma_{7}\left(\frac{n}{8}\right)-\frac{243}{55760} \sigma_{7}\left(\frac{n}{12}\right)+\frac{3888}{3485} \sigma_{7}\left(\frac{n}{24}\right)-\frac{122444543}{4186080} a_{1}(n) \\
& -\frac{954511}{5130} a_{1}\left(\frac{n}{2}\right)+\frac{26975007}{51680} a_{1}\left(\frac{n}{3}\right)+\frac{107032096}{130815} a_{1}\left(\frac{n}{4}\right)+\frac{1653147}{190} a_{1}\left(\frac{n}{6}\right)+\frac{61017696}{1615} a_{1}\left(\frac{n}{12}\right) \\
& -\frac{451759}{70110} \Delta_{3,8}(n)-\frac{30568369}{140220} \Delta_{3,8}\left(\frac{n}{2}\right)+\frac{361012364}{35055} \Delta_{3,8}\left(\frac{n}{4}\right)-\frac{419526272}{35055} \Delta_{3,8}\left(\frac{n}{8}\right) \\
& +\frac{54928}{1539} a_{4}(n)-\frac{63920}{513} a_{4}\left(\frac{n}{2}\right)-\frac{346624}{81} a_{4}\left(\frac{n}{4}\right)-\frac{21}{2} a_{2}(n)+\frac{26433}{38} a_{2}\left(\frac{n}{3}\right)+\frac{33}{2} a_{3}(n) \\
& -\frac{81837}{38} a_{3}\left(\frac{n}{3}\right)+\frac{202941}{19} a_{5}(n)+\frac{974208}{19} a_{5}\left(\frac{n}{2}\right)-\frac{171}{2} a_{6}(n)+\frac{38240}{19} a_{6}\left(\frac{n}{2}\right)-\frac{109120}{19} a_{7}(n) \\
& +\frac{58304}{19} a_{8}(n)+\frac{184960}{19} a_{9}(n) \\
& N\left(1^{3}, 2^{9}, 3^{3}, 6 ; n\right)=\frac{73}{5352960} \sigma_{7}(n)-\frac{73}{5352960} \sigma_{7}\left(\frac{n}{2}\right)-\frac{243}{1784320} \sigma_{7}\left(\frac{n}{3}\right)+\frac{73}{167280} \sigma_{7}\left(\frac{n}{4}\right) \\
& +\frac{243}{1784320} \sigma_{7}\left(\frac{n}{6}\right)-\frac{1168}{10455} \sigma_{7}\left(\frac{n}{8}\right)-\frac{243}{55760} \sigma_{7}\left(\frac{n}{12}\right)+\frac{3888}{3485} \sigma_{7}\left(\frac{n}{24}\right)-\frac{138345527}{2093040} a_{1}(n) \\
& -\frac{615784}{2565} a_{1}\left(\frac{n}{2}\right)+\frac{42849063}{25840} a_{1}\left(\frac{n}{3}\right)+\frac{56631584}{130815} a_{1}\left(\frac{n}{4}\right)+\frac{2570148}{95} a_{1}\left(\frac{n}{6}\right)+\frac{40493664}{1615} a_{1}\left(\frac{n}{12}\right) \\
& -\frac{1435247}{70110} \Delta_{3,8}(n)-\frac{18305761}{70110} \Delta_{3,8}\left(\frac{n}{2}\right)+\frac{293920682}{7011} \Delta_{3,8}\left(\frac{n}{4}\right)-\frac{30817408}{35055} \Delta_{3,8}\left(\frac{n}{8}\right) \\
& +\frac{133220}{1539} a_{4}(n)-\frac{374104}{513} a_{4}\left(\frac{n}{2}\right)-\frac{1942016}{81} a_{4}\left(\frac{n}{4}\right)-15 a_{2}(n)+\frac{58023}{19} a_{2}\left(\frac{n}{3}\right)+21 a_{3}(n) \\
& -\frac{141129}{19} a_{3}\left(\frac{n}{3}\right)+\frac{582138}{19} a_{5}(n)+\frac{5145216}{19} a_{5}\left(\frac{n}{2}\right)-123 a_{6}(n)+\frac{191008}{19} a_{6}\left(\frac{n}{2}\right) \\
& -\frac{250064}{19} a_{7}(n)+\frac{222016}{19} a_{8}(n)+\frac{345152}{19} a_{9}(n), \\
& N\left(1^{3}, 2^{11}, 3,6 ; n\right)=\frac{1093}{53529600} \sigma_{7}(n)-\frac{1093}{53529600} \sigma_{7}\left(\frac{n}{2}\right)+\frac{729}{17843200} \sigma_{7}\left(\frac{n}{3}\right)-\frac{1093}{836400} \sigma_{7}\left(\frac{n}{4}\right) \\
& -\frac{729}{17843200} \sigma_{7}\left(\frac{n}{6}\right)+\frac{17488}{52275} \sigma_{7}\left(\frac{n}{8}\right)-\frac{729}{278800} \sigma_{7}\left(\frac{n}{12}\right)+\frac{11664}{17425} \sigma_{7}\left(\frac{n}{24}\right)+\frac{339730787}{6976800} a_{1}(n) \\
& +\frac{171397}{5130} a_{1}\left(\frac{n}{2}\right)-\frac{440015409}{258400} a_{1}\left(\frac{n}{3}\right)+\frac{178759456}{218025} a_{1}\left(\frac{n}{4}\right)-\frac{5349969}{190} a_{1}\left(\frac{n}{6}\right)+\frac{373115808}{8075} a_{1}\left(\frac{n}{12}\right) \\
& +\frac{3275313}{155800} \Delta_{3,8}(n)-\frac{2057404}{19475} \Delta_{3,8}\left(\frac{n}{2}\right)-\frac{889254964}{19475} \Delta_{3,8}\left(\frac{n}{4}\right)-\frac{396119424}{19475} \Delta_{3,8}\left(\frac{n}{8}\right) \\
& -\frac{178849}{2565} a_{4}(n)+\frac{523088}{513} a_{4}\left(\frac{n}{2}\right)+\frac{3812864}{135} a_{4}\left(\frac{n}{4}\right)-\frac{1}{2} a_{2}(n)-\frac{148959}{38} a_{2}\left(\frac{n}{3}\right)+\frac{13}{2} a_{3}(n) \\
& +\frac{315171}{38} a_{3}\left(\frac{n}{3}\right)-\frac{556443}{19} a_{5}(n)-\frac{5992704}{19} a_{5}\left(\frac{n}{2}\right)+\frac{17}{2} a_{6}(n)-\frac{221024}{19} a_{6}\left(\frac{n}{2}\right) \\
& +\frac{201024}{19} a_{7}(n)-\frac{224064}{19} a_{8}(n)-\frac{230400}{19} a_{9}(n),
\end{aligned}
$$




$$
\begin{aligned}
& N\left(1^{4}, 6^{12} ; n\right)=\frac{1}{13382400} \sigma_{7}(n)-\frac{1}{13382400} \sigma_{7}\left(\frac{n}{2}\right)+\frac{273}{4460800} \sigma_{7}\left(\frac{n}{3}\right)-\frac{1}{209100} \sigma_{7}\left(\frac{n}{4}\right) \\
& -\frac{273}{4460800} \sigma_{7}\left(\frac{n}{6}\right)+\frac{64}{52275} \sigma_{7}\left(\frac{n}{8}\right)-\frac{273}{69700} \sigma_{7}\left(\frac{n}{12}\right)+\frac{17472}{17425} \sigma_{7}\left(\frac{n}{24}\right)-\frac{153792059}{15697800} a_{1}(n) \\
& -\frac{4696882}{23085} a_{1}\left(\frac{n}{2}\right)-\frac{7142343}{64600} a_{1}\left(\frac{n}{3}\right)+\frac{864113024}{654075} a_{1}\left(\frac{n}{4}\right)-\frac{60954}{95} a_{1}\left(\frac{n}{6}\right)+\frac{316738944}{8075} a_{1}\left(\frac{n}{12}\right) \\
& +\frac{2870179}{2103300} \Delta_{3,8}(n)-\frac{65343281}{350550} \Delta_{3,8}\left(\frac{n}{2}\right)-\frac{5453804824}{525825} \Delta_{3,8}\left(\frac{n}{4}\right)-\frac{4127615488}{175275} \Delta_{3,8}\left(\frac{n}{8}\right) \\
& +\frac{194662}{23085} a_{4}(n)+\frac{974048}{4617} a_{4}\left(\frac{n}{2}\right)+\frac{3905536}{405} a_{4}\left(\frac{n}{4}\right)-8 a_{2}(n)-\frac{7758}{19} a_{2}\left(\frac{n}{3}\right)+16 a_{3}(n) \\
& +\frac{7758}{19} a_{3}\left(\frac{n}{3}\right)-\frac{6124}{19} a_{5}(n)-\frac{1918208}{19} a_{5}\left(\frac{n}{2}\right)-66 a_{6}(n)-\frac{66688}{19} a_{6}\left(\frac{n}{2}\right) \\
& -\frac{151232}{57} a_{7}(n)-\frac{59392}{19} a_{8}(n)+\frac{147968}{19} a_{9}(n) \text {, } \\
& N\left(1^{4}, 3^{4}, 6^{8} ; n\right)=\frac{1}{6691200} \sigma_{7}(n)-\frac{1}{6691200} \sigma_{7}\left(\frac{n}{2}\right)+\frac{273}{2230400} \sigma_{7}\left(\frac{n}{3}\right)-\frac{1}{209100} \sigma_{7}\left(\frac{n}{4}\right) \\
& -\frac{273}{2230400} \sigma_{7}\left(\frac{n}{6}\right)+\frac{64}{52275} \sigma_{7}\left(\frac{n}{8}\right)-\frac{273}{69700} \sigma_{7}\left(\frac{n}{12}\right)+\frac{17472}{17425} \sigma_{7}\left(\frac{n}{24}\right)-\frac{71664889}{7848900} a_{1}(n) \\
& -\frac{4546024}{23085} a_{1}\left(\frac{n}{2}\right)-\frac{3822753}{32300} a_{1}\left(\frac{n}{3}\right)+\frac{841700224}{654075} a_{1}\left(\frac{n}{4}\right)-\frac{77928}{95} a_{1}\left(\frac{n}{6}\right)+\frac{325551744}{8075} a_{1}\left(\frac{n}{12}\right) \\
& +\frac{767917}{525825} \Delta_{3,8}(n)-\frac{9900592}{58425} \Delta_{3,8}\left(\frac{n}{2}\right)-\frac{5367891784}{525825} \Delta_{3,8}\left(\frac{n}{4}\right)-\frac{4022655488}{175275} \Delta_{3,8}\left(\frac{n}{8}\right) \\
& +\frac{177064}{23085} a_{4}(n)+\frac{922784}{4617} a_{4}\left(\frac{n}{2}\right)+\frac{3823616}{405} a_{4}\left(\frac{n}{4}\right)-8 a_{2}(n)-\frac{8406}{19} a_{2}\left(\frac{n}{3}\right)+16 a_{3}(n) \\
& +\frac{9090}{19} a_{3}\left(\frac{n}{3}\right)-\frac{7000}{19} a_{5}(n)-\frac{1883136}{19} a_{5}\left(\frac{n}{2}\right)-60 a_{6}(n)-\frac{65408}{19} a_{6}\left(\frac{n}{2}\right) \\
& -\frac{141248}{57} a_{7}(n)-\frac{58112}{19} a_{8}(n)+\frac{140544}{19} a_{9}(n) \text {, } \\
& N\left(1^{4}, 3^{4}, 6^{8} ; n\right)=\frac{1}{3345600} \sigma_{7}(n)-\frac{1}{3345600} \sigma_{7}\left(\frac{n}{2}\right)+\frac{273}{1115200} \sigma_{7}\left(\frac{n}{3}\right)-\frac{1}{209100} \sigma_{7}\left(\frac{n}{4}\right) \\
& -\frac{273}{1115200} \sigma_{7}\left(\frac{n}{6}\right)+\frac{64}{52275} \sigma_{7}\left(\frac{n}{8}\right)-\frac{273}{69700} \sigma_{7}\left(\frac{n}{12}\right)+\frac{17472}{17425} \sigma_{7}\left(\frac{n}{24}\right)-\frac{3731371}{436050} a_{1}(n) \\
& -\frac{97480}{513} a_{1}\left(\frac{n}{2}\right)-\frac{2060703}{16150} a_{1}\left(\frac{n}{3}\right)+\frac{823291264}{654075} a_{1}\left(\frac{n}{4}\right)-\frac{17928}{19} a_{1}\left(\frac{n}{6}\right)+\frac{334560384}{8075} a_{1}\left(\frac{n}{12}\right) \\
& +\frac{91951}{58425} \Delta_{3,8}(n)-\frac{3074258}{19475} \Delta_{3,8}\left(\frac{n}{2}\right)-\frac{1773707968}{175275} \Delta_{3,8}\left(\frac{n}{4}\right)-\frac{3938739968}{175275} \Delta_{3,8}\left(\frac{n}{8}\right) \\
& +\frac{17912}{2565} a_{4}(n)+\frac{98560}{513} a_{4}\left(\frac{n}{2}\right)+\frac{3777536}{405} a_{4}\left(\frac{n}{4}\right)-8 a_{2}(n)-\frac{9000}{19} a_{2}\left(\frac{n}{3}\right)+16 a_{3}(n) \\
& +\frac{10368}{19} a_{3}\left(\frac{n}{3}\right)-\frac{8848}{19} a_{5}(n)-\frac{1865984}{19} a_{5}\left(\frac{n}{2}\right)-56 a_{6}(n)-\frac{64640}{19} a_{6}\left(\frac{n}{2}\right) \\
& -\frac{44032}{19} a_{7}(n)-\frac{57344}{19} a_{8}(n)+\frac{134144}{19} a_{9}(n) \text {, }
\end{aligned}
$$




$$
\begin{aligned}
& N\left(1^{4}, 3^{6}, 6^{6} ; n\right)=\frac{1}{1672800} \sigma_{7}(n)-\frac{1}{1672800} \sigma_{7}\left(\frac{n}{2}\right)+\frac{273}{557600} \sigma_{7}\left(\frac{n}{3}\right)-\frac{1}{209100} \sigma_{7}\left(\frac{n}{4}\right) \\
& -\frac{273}{557600} \sigma_{7}\left(\frac{n}{6}\right)+\frac{64}{52275} \sigma_{7}\left(\frac{n}{8}\right)-\frac{273}{69700} \sigma_{7}\left(\frac{n}{12}\right)+\frac{17472}{17425} \sigma_{7}\left(\frac{n}{24}\right)-\frac{15292294}{1962225} a_{1}(n) \\
& -\frac{4203448}{23085} a_{1}\left(\frac{n}{2}\right)-\frac{1083288}{8075} a_{1}\left(\frac{n}{3}\right)+\frac{808886144}{654075} a_{1}\left(\frac{n}{4}\right)-\frac{100536}{95} a_{1}\left(\frac{n}{6}\right)+\frac{343764864}{8075} a_{1}\left(\frac{n}{12}\right) \\
& +\frac{869353}{525825} \Delta_{3,8}(n)-\frac{23197154}{175275} \Delta_{3,8}\left(\frac{n}{2}\right)-\frac{5300227024}{525825} \Delta_{3,8}\left(\frac{n}{4}\right)-\frac{3875868928}{175275} \Delta_{3,8}\left(\frac{n}{8}\right) \\
& +\frac{141736}{23085} a_{4}(n)+\frac{797504}{4617} a_{4}\left(\frac{n}{2}\right)+\frac{3767296}{405} a_{4}\left(\frac{n}{4}\right)-8 a_{2}(n)-\frac{9540}{19} a_{2}\left(\frac{n}{3}\right)+16 a_{3}(n) \\
& +\frac{11592}{19} a_{3}\left(\frac{n}{3}\right)-\frac{8704}{19} a_{5}(n)-\frac{1866752}{19} a_{5}\left(\frac{n}{2}\right)-48 a_{6}(n)-\frac{64384}{19} a_{6}\left(\frac{n}{2}\right)-\frac{123776}{57} a_{7}(n) \\
& -\frac{57088}{19} a_{8}(n)+\frac{128768}{19} a_{9}(n) \text {, } \\
& N\left(1^{4}, 3^{8}, 6^{4} ; n\right)=\frac{1}{836400} \sigma_{7}(n)-\frac{1}{836400} \sigma_{7}\left(\frac{n}{2}\right)+\frac{273}{278800} \sigma_{7}\left(\frac{n}{3}\right)-\frac{1}{209100} \sigma_{7}\left(\frac{n}{4}\right) \\
& -\frac{273}{278800} \sigma_{7}\left(\frac{n}{6}\right)+\frac{64}{52275} \sigma_{7}\left(\frac{n}{8}\right)-\frac{273}{69700} \sigma_{7}\left(\frac{n}{12}\right)+\frac{17472}{17425} \sigma_{7}\left(\frac{n}{24}\right)-\frac{12991628}{1962225} a_{1}(n) \\
& -\frac{3938672}{23085} a_{1}\left(\frac{n}{2}\right)-\frac{1089456}{8075} a_{1}\left(\frac{n}{3}\right)+\frac{264827008}{218025} a_{1}\left(\frac{n}{4}\right)-\frac{110064}{95} a_{1}\left(\frac{n}{6}\right)+\frac{352969344}{8075} a_{1}\left(\frac{n}{12}\right) \\
& +\frac{872786}{525825} \Delta_{3,8}(n)-\frac{14094508}{175275} \Delta_{3,8}\left(\frac{n}{2}\right)-\frac{5238759824}{525825} \Delta_{3,8}\left(\frac{n}{4}\right)-\frac{423666432}{19475} \Delta_{3,8}\left(\frac{n}{8}\right) \\
& +\frac{114512}{23085} a_{4}(n)+\frac{602176}{4617} a_{4}\left(\frac{n}{2}\right)+\frac{1252352}{135} a_{4}\left(\frac{n}{4}\right)-8 a_{2}(n)-\frac{10080}{19} a_{2}\left(\frac{n}{3}\right) \\
& +16 a_{3}(n)+\frac{12816}{19} a_{3}\left(\frac{n}{3}\right)-\frac{4608}{19} a_{5}(n)-\frac{1867520}{19} a_{5}\left(\frac{n}{2}\right)-32 a_{6}(n)-\frac{64128}{19} a_{6}\left(\frac{n}{2}\right) \\
& -\frac{115456}{57} a_{7}(n)-\frac{56832}{19} a_{8}(n)+\frac{123392}{19} a_{9}(n) \text {, } \\
& N\left(1^{4}, 3^{10}, 6^{2} ; n\right)=\frac{1}{418200} \sigma_{7}(n)-\frac{1}{418200} \sigma_{7}\left(\frac{n}{2}\right)+\frac{273}{139400} \sigma_{7}\left(\frac{n}{3}\right)-\frac{1}{209100} \sigma_{7}\left(\frac{n}{4}\right) \\
& -\frac{273}{139400} \sigma_{7}\left(\frac{n}{6}\right)+\frac{64}{52275} \sigma_{7}\left(\frac{n}{8}\right)-\frac{273}{69700} \sigma_{7}\left(\frac{n}{12}\right)+\frac{17472}{17425} \sigma_{7}\left(\frac{n}{24}\right)-\frac{9832916}{1962225} a_{1}(n) \\
& -\frac{3652304}{23085} a_{1}\left(\frac{n}{2}\right)-\frac{757032}{8075} a_{1}\left(\frac{n}{3}\right)+\frac{264827008}{218025} a_{1}\left(\frac{n}{4}\right)-\frac{93648}{95} a_{1}\left(\frac{n}{6}\right)+\frac{352969344}{8075} a_{1}\left(\frac{n}{12}\right) \\
& +\frac{836192}{525825} \Delta_{3,8}(n)+\frac{473336}{19475} \Delta_{3,8}\left(\frac{n}{2}\right)-\frac{5062269584}{525825} \Delta_{3,8}\left(\frac{n}{4}\right)-\frac{423666432}{19475} \Delta_{3,8}\left(\frac{n}{8}\right) \\
& +\frac{78944}{23085} a_{4}(n)+\frac{193600}{4617} a_{4}\left(\frac{n}{2}\right)+\frac{1252352}{135} a_{4}\left(\frac{n}{4}\right)-8 a_{2}(n)-\frac{10080}{19} a_{2}\left(\frac{n}{3}\right) \\
& +16 a_{3}(n)+\frac{12816}{19} a_{3}\left(\frac{n}{3}\right)+\frac{11200}{19} a_{5}(n)-\frac{1867520}{19} a_{5}\left(\frac{n}{2}\right)-\frac{64128}{19} a_{6}\left(\frac{n}{2}\right) \\
& -\frac{115456}{57} a_{7}(n)-\frac{56832}{19} a_{8}(n)+\frac{123392}{19} a_{9}(n) \text {, }
\end{aligned}
$$




$$
\begin{aligned}
& N\left(1^{4}, 3^{12} ; n\right)=\frac{1}{209100} \sigma_{7}(n)-\frac{1}{104550} \sigma_{7}\left(\frac{n}{2}\right)+\frac{273}{69700} \sigma_{7}\left(\frac{n}{3}\right) \\
& +\frac{64}{52275} \sigma_{7}\left(\frac{n}{4}\right)-\frac{273}{34850} \sigma_{7}\left(\frac{n}{6}\right)+\frac{17472}{17425} \sigma_{7}\left(\frac{n}{12}\right)+\frac{93824}{34425} a_{1}(n) \\
& +\frac{2093056}{34425} a_{1}\left(\frac{n}{2}\right)-\frac{3456}{425} a_{1}\left(\frac{n}{3}\right)+\frac{884736}{425} a_{1}\left(\frac{n}{6}\right)+\frac{25312}{9225} \Delta_{3,8}(n) \\
& -\frac{30592}{1845} \Delta_{3,8}\left(\frac{n}{2}\right)+\frac{10006528}{9225} \Delta_{3,8}\left(\frac{n}{4}\right)+\frac{1024}{405} a_{4}(n)-\frac{16384}{405} a_{4}\left(\frac{n}{2}\right) \text {, } \\
& N\left(1^{4}, 2^{2}, 6^{10} ; n\right)=\frac{7}{26764800} \sigma_{7}(n)-\frac{7}{26764800} \sigma_{7}\left(\frac{n}{2}\right)-\frac{549}{8921600} \sigma_{7}\left(\frac{n}{3}\right) \\
& +\frac{7}{418200} \sigma_{7}\left(\frac{n}{4}\right)+\frac{549}{8921600} \sigma_{7}\left(\frac{n}{6}\right)-\frac{224}{52275} \sigma_{7}\left(\frac{n}{8}\right)-\frac{549}{139400} \sigma_{7}\left(\frac{n}{12}\right) \\
& +\frac{17568}{17425} \sigma_{7}\left(\frac{n}{24}\right)-\frac{108795931}{10465200} a_{1}(n)-\frac{1467371}{7695} a_{1}\left(\frac{n}{2}\right)-\frac{10885761}{129200} a_{1}\left(\frac{n}{3}\right) \\
& +\frac{802187456}{654075} a_{1}\left(\frac{n}{4}\right)-\frac{61371}{95} a_{1}\left(\frac{n}{6}\right)+\frac{331897536}{8075} a_{1}\left(\frac{n}{12}\right)+\frac{1459061}{1402200} \Delta_{3,8}(n) \\
& -\frac{125975777}{701100} \Delta_{3,8}\left(\frac{n}{2}\right)-\frac{1467072052}{175275} \Delta_{3,8}\left(\frac{n}{4}\right)-\frac{3791510272}{175275} \Delta_{3,8}\left(\frac{n}{8}\right) \\
& +\frac{71989}{7695} a_{4}(n)+\frac{288400}{1539} a_{4}\left(\frac{n}{2}\right)+\frac{3257344}{405} a_{4}\left(\frac{n}{4}\right)-9 a_{2}(n)-\frac{8667}{19} a_{2}\left(\frac{n}{3}\right) \\
& +17 a_{3}(n)+\frac{8667}{19} a_{3}\left(\frac{n}{3}\right)+\frac{5982}{19} a_{5}(n)-\frac{1596288}{19} a_{5}\left(\frac{n}{2}\right)-63 a_{6}(n) \\
& -\frac{55232}{19} a_{6}\left(\frac{n}{2}\right)-\frac{47904}{19} a_{7}(n)-\frac{44288}{19} a_{8}(n)+\frac{132352}{19} a_{9}(n) \text {, } \\
& N\left(1^{4}, 2^{2}, 3^{2}, 6^{8} ; n\right)=\frac{7}{13382400} \sigma_{7}(n)-\frac{7}{13382400} \sigma_{7}\left(\frac{n}{2}\right)-\frac{549}{4460800} \sigma_{7}\left(\frac{n}{3}\right) \\
& +\frac{7}{418200} \sigma_{7}\left(\frac{n}{4}\right)+\frac{549}{4460800} \sigma_{7}\left(\frac{n}{6}\right)-\frac{224}{52275} \sigma_{7}\left(\frac{n}{8}\right)-\frac{549}{139400} \sigma_{7}\left(\frac{n}{12}\right) \\
& +\frac{17568}{17425} \sigma_{7}\left(\frac{n}{24}\right)-\frac{64982171}{5232600} a_{1}(n)-\frac{1386928}{7695} a_{1}\left(\frac{n}{2}\right)-\frac{2268801}{64600} a_{1}\left(\frac{n}{3}\right) \\
& +\frac{28149568}{24225} a_{1}\left(\frac{n}{4}\right)+\frac{54792}{95} a_{1}\left(\frac{n}{6}\right)+\frac{401322816}{8075} a_{1}\left(\frac{n}{12}\right)+\frac{304501}{701100} \Delta_{3,8}(n) \\
& -\frac{23174839}{116850} \Delta_{3,8}\left(\frac{n}{2}\right)-\frac{1041029572}{175275} \Delta_{3,8}\left(\frac{n}{4}\right)-\frac{1178854144}{58425} \Delta_{3,8}\left(\frac{n}{8}\right) \\
& +\frac{92218}{7695} a_{4}(n)+\frac{247376}{1539} a_{4}\left(\frac{n}{2}\right)+\frac{31744}{5} a_{4}\left(\frac{n}{4}\right)-10 a_{2}(n)-\frac{7992}{19} a_{2}\left(\frac{n}{3}\right) \\
& +18 a_{3}(n)+\frac{5940}{19} a_{3}\left(\frac{n}{3}\right)+\frac{23244}{19} a_{5}(n)-\frac{1263360}{19} a_{5}\left(\frac{n}{2}\right)-70 a_{6}(n) \\
& -\frac{42176}{19} a_{6}\left(\frac{n}{2}\right)-\frac{51168}{19} a_{7}(n)-\frac{28800}{19} a_{8}(n)+\frac{128128}{19} a_{9}(n) \text {, }
\end{aligned}
$$




$$
\begin{aligned}
& N\left(1^{4}, 2^{2}, 3^{4}, 6^{6} ; n\right)=\frac{7}{6691200} \sigma_{7}(n)-\frac{7}{6691200} \sigma_{7}\left(\frac{n}{2}\right)-\frac{549}{2230400} \sigma_{7}\left(\frac{n}{3}\right)+\frac{7}{418200} \sigma_{7}\left(\frac{n}{4}\right) \\
& +\frac{549}{2230400} \sigma_{7}\left(\frac{n}{6}\right)-\frac{224}{52275} \sigma_{7}\left(\frac{n}{8}\right)-\frac{549}{139400} \sigma_{7}\left(\frac{n}{12}\right)+\frac{17568}{17425} \sigma_{7}\left(\frac{n}{24}\right)-\frac{38619931}{2616300} a_{1}(n) \\
& -\frac{136256}{855} a_{1}\left(\frac{n}{2}\right)+\frac{864639}{32300} a_{1}\left(\frac{n}{3}\right)+\frac{674608576}{654075} a_{1}\left(\frac{n}{4}\right)+\frac{175896}{95} a_{1}\left(\frac{n}{6}\right)+\frac{479854656}{8075} a_{1}\left(\frac{n}{12}\right) \\
& -\frac{115339}{350550} \Delta_{3,8}(n)-\frac{37899577}{175275} \Delta_{3,8}\left(\frac{n}{2}\right)-\frac{379980832}{175275} \Delta_{3,8}\left(\frac{n}{4}\right)-\frac{3048682112}{175275} \Delta_{3,8}\left(\frac{n}{8}\right) \\
& +\frac{116116}{7695} a_{4}(n)+\frac{20608}{171} a_{4}\left(\frac{n}{2}\right)+\frac{1447424}{405} a_{4}\left(\frac{n}{4}\right)-12 a_{2}(n)-\frac{7884}{19} a_{2}\left(\frac{n}{3}\right)+20 a_{3}(n) \\
& +\frac{3780}{19} a_{3}\left(\frac{n}{3}\right)+\frac{47064}{19} a_{5}(n)-\frac{713088}{19} a_{5}\left(\frac{n}{2}\right)-76 a_{6}(n)-\frac{21312}{19} a_{6}\left(\frac{n}{2}\right) \\
& -\frac{52736}{19} a_{7}(n)-\frac{3072}{19} a_{8}(n)+\frac{113152}{19} a_{9}(n) \text {, } \\
& N\left(1^{4}, 2^{2}, 3^{6}, 6^{4} ; n\right)=\frac{7}{3345600} \sigma_{7}(n)-\frac{7}{3345600} \sigma_{7}\left(\frac{n}{2}\right)-\frac{549}{1115200} \sigma_{7}\left(\frac{n}{3}\right)+\frac{7}{418200} \sigma_{7}\left(\frac{n}{4}\right) \\
& +\frac{549}{1115200} \sigma_{7}\left(\frac{n}{6}\right)-\frac{224}{52275} \sigma_{7}\left(\frac{n}{8}\right)-\frac{549}{139400} \sigma_{7}\left(\frac{n}{12}\right)+\frac{17568}{17425} \sigma_{7}\left(\frac{n}{24}\right)-\frac{11849218}{654075} a_{1}(n) \\
& -\frac{291604}{2565} a_{1}\left(\frac{n}{2}\right)+\frac{877167}{8075} a_{1}\left(\frac{n}{3}\right)+\frac{507361216}{654075} a_{1}\left(\frac{n}{4}\right)+\frac{351828}{95} a_{1}\left(\frac{n}{6}\right)+\frac{647004096}{8075} a_{1}\left(\frac{n}{12}\right) \\
& -\frac{469093}{350550} \Delta_{3,8}(n)-\frac{44967769}{175275} \Delta_{3,8}\left(\frac{n}{2}\right)+\frac{768440648}{175275} \Delta_{3,8}\left(\frac{n}{4}\right)-\frac{2112228992}{175275} \Delta_{3,8}\left(\frac{n}{8}\right) \\
& +\frac{149692}{7695} a_{4}(n)+\frac{34208}{513} a_{4}\left(\frac{n}{2}\right)-\frac{580096}{405} a_{4}\left(\frac{n}{4}\right)-16 a_{2}(n)-\frac{9504}{19} a_{2}\left(\frac{n}{3}\right)+24 a_{3}(n) \\
& +\frac{3348}{19} a_{3}\left(\frac{n}{3}\right)+\frac{79872}{19} a_{5}(n)+\frac{269568}{19} a_{5}\left(\frac{n}{2}\right)-88 a_{6}(n)+\frac{15936}{19} a_{6}\left(\frac{n}{2}\right) \\
& -\frac{50496}{19} a_{7}(n)+\frac{43904}{19} a_{8}(n)+\frac{75136}{19} a_{9}(n), \\
& N\left(1^{4}, 2^{2}, 3^{8}, 6^{2} ; n\right)=\frac{7}{1672800} \sigma_{7}(n)-\frac{7}{1672800} \sigma_{7}\left(\frac{n}{2}\right)-\frac{549}{557600} \sigma_{7}\left(\frac{n}{3}\right)+\frac{7}{418200} \sigma_{7}\left(\frac{n}{4}\right) \\
& +\frac{549}{557600} \sigma_{7}\left(\frac{n}{6}\right)-\frac{224}{52275} \sigma_{7}\left(\frac{n}{8}\right)-\frac{549}{139400} \sigma_{7}\left(\frac{n}{12}\right)+\frac{17568}{17425} \sigma_{7}\left(\frac{n}{24}\right)-\frac{5146102}{218025} a_{1}(n) \\
& -\frac{10384}{513} a_{1}\left(\frac{n}{2}\right)+\frac{1847664}{8075} a_{1}\left(\frac{n}{3}\right)+\frac{62184512}{218025} a_{1}\left(\frac{n}{4}\right)+\frac{129816}{19} a_{1}\left(\frac{n}{6}\right)+\frac{991780416}{8075} a_{1}\left(\frac{n}{12}\right) \\
& -\frac{164701}{58425} \Delta_{3,8}(n)-\frac{6591842}{19475} \Delta_{3,8}\left(\frac{n}{2}\right)+\frac{318066952}{19475} \Delta_{3,8}\left(\frac{n}{4}\right)-\frac{110230144}{58425} \Delta_{3,8}\left(\frac{n}{8}\right) \\
& +\frac{67768}{2565} a_{4}(n)-\frac{9056}{513} a_{4}\left(\frac{n}{2}\right)-\frac{1444352}{135} a_{4}\left(\frac{n}{4}\right)-24 a_{2}(n)-\frac{14472}{19} a_{2}\left(\frac{n}{3}\right)+32 a_{3}(n) \\
& +\frac{6264}{19} a_{3}\left(\frac{n}{3}\right)+\frac{131904}{19} a_{5}(n)+\frac{2076288}{19} a_{5}\left(\frac{n}{2}\right)-112 a_{6}(n)+\frac{84928}{19} a_{6}\left(\frac{n}{2}\right) \\
& -\frac{41600}{19} a_{7}(n)+\frac{132352}{19} a_{8}(n)+-\frac{6912}{19} a_{9}(n) \text {, }
\end{aligned}
$$




$$
\begin{aligned}
& N\left(1^{4}, 2^{2}, 3^{10} ; n\right)=\frac{7}{836400} \sigma_{7}(n)-\frac{7}{836400} \sigma_{7}\left(\frac{n}{2}\right)-\frac{549}{278800} \sigma_{7}\left(\frac{n}{3}\right)+\frac{7}{418200} \sigma_{7}\left(\frac{n}{4}\right) \\
& +\frac{549}{278800} \sigma_{7}\left(\frac{n}{6}\right)-\frac{224}{52275} \sigma_{7}\left(\frac{n}{8}\right)-\frac{549}{139400} \sigma_{7}\left(\frac{n}{12}\right)+\frac{17568}{17425} \sigma_{7}\left(\frac{n}{24}\right)-\frac{19501742}{654075} a_{1}(n) \\
& +\frac{2232184}{7695} a_{1}\left(\frac{n}{2}\right)+\frac{3097098}{8075} a_{1}\left(\frac{n}{3}\right)-\frac{99923776}{72675} a_{1}\left(\frac{n}{4}\right)+\frac{1314864}{95} a_{1}\left(\frac{n}{6}\right)+\frac{1522898496}{8075} a_{1}\left(\frac{n}{12}\right) \\
& -\frac{827896}{175275} \Delta_{3,8}(n)-\frac{36740092}{58425} \Delta_{3,8}\left(\frac{n}{2}\right)+\frac{7768997128}{175275} \Delta_{3,8}\left(\frac{n}{4}\right)+\frac{591722112}{19475} \Delta_{3,8}\left(\frac{n}{8}\right) \\
& +\frac{296528}{7695} a_{4}(n)-\frac{180512}{1539} a_{4}\left(\frac{n}{2}\right)-\frac{1508864}{45} a_{4}\left(\frac{n}{4}\right)-36 a_{2}(n)-\frac{21924}{19} a_{2}\left(\frac{n}{3}\right)+40 a_{3}(n) \\
& +\frac{11664}{19} a_{3}\left(\frac{n}{3}\right)+\frac{188064}{19} a_{5}(n)+\frac{6533760}{19} a_{5}\left(\frac{n}{2}\right)-192 a_{6}(n)+\frac{252864}{19} a_{6}\left(\frac{n}{2}\right) \\
& +\frac{8832}{19} a_{7}(n)+\frac{329472}{19} a_{8}(n)-\frac{255232}{19} a_{9}(n) \text {, } \\
& N\left(1^{4}, 2^{4}, 6^{8} ; n\right)=\frac{1}{1338240} \sigma_{7}(n)-\frac{1}{1338240} \sigma_{7}\left(\frac{n}{2}\right)+\frac{27}{446080} \sigma_{7}\left(\frac{n}{3}\right)-\frac{1}{20910} \sigma_{7}\left(\frac{n}{4}\right) \\
& -\frac{27}{446080} \sigma_{7}\left(\frac{n}{6}\right)+\frac{128}{10455} \sigma_{7}\left(\frac{n}{8}\right)-\frac{27}{6970} \sigma_{7}\left(\frac{n}{12}\right)+\frac{3456}{3485} \sigma_{7}\left(\frac{n}{24}\right)-\frac{61573}{6460} a_{1}(n) \\
& -\frac{562928}{2565} a_{1}\left(\frac{n}{2}\right)-\frac{691173}{6460} a_{1}\left(\frac{n}{3}\right)+\frac{61152512}{43605} a_{1}\left(\frac{n}{4}\right)-\frac{59724}{95} a_{1}\left(\frac{n}{6}\right)+\frac{61122816}{1615} a_{1}\left(\frac{n}{12}\right) \\
& +\frac{10287}{7790} \Delta_{3,8}(n)-\frac{367121}{2337} \Delta_{3,8}\left(\frac{n}{2}\right)-\frac{46659184}{3895} \Delta_{3,8}\left(\frac{n}{4}\right)-\frac{294616064}{11685} \Delta_{3,8}\left(\frac{n}{8}\right) \\
& +\frac{156}{19} a_{4}(n)+\frac{103744}{513} a_{4}\left(\frac{n}{2}\right)+\frac{299008}{27} a_{4}\left(\frac{n}{4}\right)-6 a_{2}(n)-\frac{6804}{19} a_{2}\left(\frac{n}{3}\right)+14 a_{3}(n) \\
& +\frac{6804}{19} a_{3}\left(\frac{n}{3}\right)-\frac{6120}{19} a_{5}(n)-\frac{2207232}{19} a_{5}\left(\frac{n}{2}\right)-60 a_{6}(n)-\frac{76544}{19} a_{6}\left(\frac{n}{2}\right) \\
& -\frac{52608}{19} a_{7}(n)-\frac{71680}{19} a_{8}(n)+\frac{162816}{19} a_{9}(n) \text {, } \\
& N\left(1^{4}, 2^{4}, 3^{2}, 6^{6} ; n\right)=\frac{1}{669120} \sigma_{7}(n)-\frac{1}{669120} \sigma_{7}\left(\frac{n}{2}\right)+\frac{27}{223040} \sigma_{7}\left(\frac{n}{3}\right)-\frac{1}{20910} \sigma_{7}\left(\frac{n}{4}\right) \\
& -\frac{27}{223040} \sigma_{7}\left(\frac{n}{6}\right)+\frac{128}{10455} \sigma_{7}\left(\frac{n}{8}\right)-\frac{27}{6970} \sigma_{7}\left(\frac{n}{12}\right)+\frac{3456}{3485} \sigma_{7}\left(\frac{n}{24}\right)-\frac{577628}{130815} a_{1}(n) \\
& -\frac{592744}{2565} a_{1}\left(\frac{n}{2}\right)-\frac{392328}{1615} a_{1}\left(\frac{n}{3}\right)+\frac{195799808}{130815} a_{1}\left(\frac{n}{4}\right)-\frac{346392}{95} a_{1}\left(\frac{n}{6}\right)+\frac{36407808}{1615} a_{1}\left(\frac{n}{12}\right) \\
& +\frac{210217}{70110} \Delta_{3,8}(n)-\frac{4585691}{35055} \Delta_{3,8}\left(\frac{n}{2}\right)-\frac{603167888}{35055} \Delta_{3,8}\left(\frac{n}{4}\right)-\frac{967123456}{35055} \Delta_{3,8}\left(\frac{n}{8}\right) \\
& +\frac{2180}{1539} a_{4}(n)+\frac{142784}{513} a_{4}\left(\frac{n}{2}\right)+\frac{1177600}{81} a_{4}\left(\frac{n}{4}\right)-4 a_{2}(n)-\frac{10098}{19} a_{2}\left(\frac{n}{3}\right)+12 a_{3}(n) \\
& +\frac{16254}{19} a_{3}\left(\frac{n}{3}\right)-\frac{52848}{19} a_{5}(n)-\frac{2899200}{19} a_{5}\left(\frac{n}{2}\right)-48 a_{6}(n)-\frac{103680}{19} a_{6}\left(\frac{n}{2}\right) \\
& -\frac{38528}{19} a_{7}(n)-\frac{103680}{19} a_{8}(n)+\frac{158720}{19} a_{9}(n) \text {, }
\end{aligned}
$$




$$
\begin{aligned}
& N\left(1^{4}, 2^{4}, 3^{4}, 6^{4} ; n\right)=\frac{1}{334560} \sigma_{7}(n)-\frac{1}{334560} \sigma_{7}\left(\frac{n}{2}\right)+\frac{27}{111520} \sigma_{7}\left(\frac{n}{3}\right)-\frac{1}{20910} \sigma_{7}\left(\frac{n}{4}\right) \\
& -\frac{27}{111520} \sigma_{7}\left(\frac{n}{6}\right)+\frac{128}{10455} \sigma_{7}\left(\frac{n}{8}\right)-\frac{27}{6970} \sigma_{7}\left(\frac{n}{12}\right)+\frac{3456}{3485} \sigma_{7}\left(\frac{n}{24}\right)+\frac{207107}{130815} a_{1}(n) \\
& -\frac{135944}{513} a_{1}\left(\frac{n}{2}\right)-\frac{648333}{1615} a_{1}\left(\frac{n}{3}\right)+\frac{45571328}{26163} a_{1}\left(\frac{n}{4}\right)-\frac{126936}{19} a_{1}\left(\frac{n}{6}\right)+\frac{1633536}{323} a_{1}\left(\frac{n}{12}\right) \\
& +\frac{173686}{35055} \Delta_{3,8}(n)-\frac{2932084}{35055} \Delta_{3,8}\left(\frac{n}{2}\right)-\frac{177761600}{7011} \Delta_{3,8}\left(\frac{n}{4}\right)-\frac{231239680}{7011} \Delta_{3,8}\left(\frac{n}{8}\right) \\
& -\frac{10064}{1539} a_{4}(n)+\frac{187136}{513} a_{4}\left(\frac{n}{2}\right)+\frac{1654784}{81} a_{4}\left(\frac{n}{4}\right)-\frac{12096}{19} a_{2}\left(\frac{n}{3}\right)+8 a_{3}(n) \\
& +\frac{24408}{19} a_{3}\left(\frac{n}{3}\right)-\frac{110592}{19} a_{5}(n)-\frac{4079616}{19} a_{5}\left(\frac{n}{2}\right)-32 a_{6}(n)-\frac{147968}{19} a_{6}\left(\frac{n}{2}\right) \\
& -\frac{28672}{19} a_{7}(n)-\frac{157696}{19} a_{8}(n)+\frac{179200}{19} a_{9}(n) \text {, } \\
& N\left(1^{4}, 2^{4}, 3^{6}, 6^{2} ; n\right)=\frac{1}{167280} \sigma_{7}(n)-\frac{1}{167280} \sigma_{7}\left(\frac{n}{2}\right)+\frac{27}{55760} \sigma_{7}\left(\frac{n}{3}\right)-\frac{1}{20910} \sigma_{7}\left(\frac{n}{4}\right) \\
& -\frac{27}{55760} \sigma_{7}\left(\frac{n}{6}\right)+\frac{128}{10455} \sigma_{7}\left(\frac{n}{8}\right)-\frac{27}{6970} \sigma_{7}\left(\frac{n}{12}\right)+\frac{3456}{3485} \sigma_{7}\left(\frac{n}{24}\right)+\frac{261982}{26163} a_{1}(n) \\
& -\frac{865664}{2565} a_{1}\left(\frac{n}{2}\right)-\frac{192258}{323} a_{1}\left(\frac{n}{3}\right)+\frac{286112512}{130815} a_{1}\left(\frac{n}{4}\right)-\frac{1005552}{95} a_{1}\left(\frac{n}{6}\right)-\frac{53169408}{1615} a_{1}\left(\frac{n}{12}\right) \\
& +\frac{51500}{7011} \Delta_{3,8}(n)+\frac{794888}{35055} \Delta_{3,8}\left(\frac{n}{2}\right)-\frac{1350603136}{35055} \Delta_{3,8}\left(\frac{n}{4}\right)-\frac{1493057024}{35055} \Delta_{3,8}\left(\frac{n}{8}\right) \\
& -\frac{26720}{1539} a_{4}(n)+\frac{236032}{513} a_{4}\left(\frac{n}{2}\right)+\frac{2459648}{81} a_{4}\left(\frac{n}{4}\right)+8 a_{2}(n)-\frac{10476}{19} a_{2}\left(\frac{n}{3}\right)+\frac{28944}{19} a_{3}\left(\frac{n}{3}\right) \\
& -\frac{184896}{19} a_{5}(n)-\frac{6047232}{19} a_{5}\left(\frac{n}{2}\right)-\frac{221696}{19} a_{6}\left(\frac{n}{2}\right)-\frac{24832}{19} a_{7}(n)-\frac{250880}{19} a_{8}(n) \\
& +\frac{239104}{19} a_{9}(n) \text {, } \\
& N\left(1^{4}, 2^{4}, 3^{8} ; n\right)=\frac{1}{83640} \sigma_{7}(n)-\frac{1}{83640} \sigma_{7}\left(\frac{n}{2}\right)+\frac{27}{27880} \sigma_{7}\left(\frac{n}{3}\right)-\frac{1}{20910} \sigma_{7}\left(\frac{n}{4}\right) \\
& -\frac{27}{27880} \sigma_{7}\left(\frac{n}{6}\right)+\frac{128}{10455} \sigma_{7}\left(\frac{n}{8}\right)-\frac{27}{6970} \sigma_{7}\left(\frac{n}{12}\right)+\frac{3456}{3485} \sigma_{7}\left(\frac{n}{24}\right)+\frac{38288}{1615} a_{1}(n) \\
& -\frac{1294576}{2565} a_{1}\left(\frac{n}{2}\right)-\frac{1378512}{1615} a_{1}\left(\frac{n}{3}\right)+\frac{26804480}{8721} a_{1}\left(\frac{n}{4}\right)-\frac{1617408}{95} a_{1}\left(\frac{n}{6}\right)-\frac{36986112}{323} a_{1}\left(\frac{n}{12}\right) \\
& +\frac{123068}{11685} \Delta_{3,8}(n)+\frac{2819912}{11685} \Delta_{3,8}\left(\frac{n}{2}\right)-\frac{737179616}{11685} \Delta_{3,8}\left(\frac{n}{4}\right)-\frac{143701504}{2337} \Delta_{3,8}\left(\frac{n}{8}\right) \\
& -\frac{1952}{57} a_{4}(n)+\frac{309632}{513} a_{4}\left(\frac{n}{2}\right)+\frac{1329152}{27} a_{4}\left(\frac{n}{4}\right)+24 a_{2}(n)-\frac{1296}{19} a_{2}\left(\frac{n}{3}\right)-16 a_{3}(n) \\
& +\frac{25920}{19} a_{3}\left(\frac{n}{3}\right)-\frac{299520}{19} a_{5}(n)-\frac{9755136}{19} a_{5}\left(\frac{n}{2}\right)+64 a_{6}(n)-\frac{362240}{19} a_{6}\left(\frac{n}{2}\right) \\
& -\frac{32256}{19} a_{7}(n)-\frac{435200}{19} a_{8}(n)+\frac{384000}{19} a_{9}(n) \text {, }
\end{aligned}
$$




$$
\begin{aligned}
& N\left(1^{4}, 2^{6}, 6^{6} ; n\right)=\frac{61}{26764800} \sigma_{7}(n)-\frac{61}{26764800} \sigma_{7}\left(\frac{n}{2}\right)-\frac{567}{8921600} \sigma_{7}\left(\frac{n}{3}\right)+\frac{61}{418200} \sigma_{7}\left(\frac{n}{4}\right) \\
& +\frac{567}{8921600} \sigma_{7}\left(\frac{n}{6}\right)-\frac{1952}{52275} \sigma_{7}\left(\frac{n}{8}\right)-\frac{567}{139400} \sigma_{7}\left(\frac{n}{12}\right)+\frac{18144}{17425} \sigma_{7}\left(\frac{n}{24}\right)-\frac{255121}{20400} a_{1}(n) \\
& -\frac{22849}{135} a_{1}\left(\frac{n}{2}\right)-\frac{314577}{6800} a_{1}\left(\frac{n}{3}\right)+\frac{11968064}{11475} a_{1}\left(\frac{n}{4}\right)-\frac{4347}{5} a_{1}\left(\frac{n}{6}\right)+\frac{19369152}{425} a_{1}\left(\frac{n}{12}\right) \\
& +\frac{14059}{24600} \Delta_{3,8}(n)-\frac{2905463}{12300} \Delta_{3,8}\left(\frac{n}{2}\right)-\frac{13772588}{3075} \Delta_{3,8}\left(\frac{n}{4}\right)-\frac{54938368}{3075} \Delta_{3,8}\left(\frac{n}{8}\right) \\
& +\frac{179}{15} a_{4}(n)+\frac{5360}{27} a_{4}\left(\frac{n}{2}\right)+\frac{646144}{135} a_{4}\left(\frac{n}{4}\right)-11 a_{2}(n)-567 a_{2}\left(\frac{n}{3}\right)+19 a_{3}(n)+567 a_{3}\left(\frac{n}{3}\right) \\
& +1134 a_{5}(n)-49536 a_{5}\left(\frac{n}{2}\right)-77 a_{6}(n)-1728 a_{6}\left(\frac{n}{2}\right)-2208 a_{7}(n)-768 a_{8}(n)+5376 a_{9}(n), \\
& N\left(1^{4}, 2^{6}, 3^{2}, 6^{4} ; n\right)=\frac{61}{13382400} \sigma_{7}(n)-\frac{61}{13382400} \sigma_{7}\left(\frac{n}{2}\right)-\frac{567}{4460800} \sigma_{7}\left(\frac{n}{3}\right)+\frac{61}{418200} \sigma_{7}\left(\frac{n}{4}\right) \\
& +\frac{567}{4460800} \sigma_{7}\left(\frac{n}{6}\right)-\frac{1952}{52275} \sigma_{7}\left(\frac{n}{8}\right)-\frac{567}{139400} \sigma_{7}\left(\frac{n}{12}\right)+\frac{18144}{17425} \sigma_{7}\left(\frac{n}{24}\right)-\frac{137445193}{5232600} a_{1}(n) \\
& -\frac{469328}{2565} a_{1}\left(\frac{n}{2}\right)+\frac{21862917}{64600} a_{1}\left(\frac{n}{3}\right)+\frac{631108928}{654075} a_{1}\left(\frac{n}{4}\right)+\frac{628056}{95} a_{1}\left(\frac{n}{6}\right)+\frac{494232768}{8075} a_{1}\left(\frac{n}{12}\right) \\
& -\frac{2928817}{701100} \Delta_{3,8}(n)-\frac{92365711}{350550} \Delta_{3,8}\left(\frac{n}{2}\right)+\frac{1168963444}{175275} \Delta_{3,8}\left(\frac{n}{4}\right)-\frac{2617812736}{175275} \Delta_{3,8}\left(\frac{n}{8}\right) \\
& +\frac{234254}{7695} a_{4}(n)-\frac{7856}{513} a_{4}\left(\frac{n}{2}\right)-\frac{785408}{405} a_{4}\left(\frac{n}{4}\right)-14 a_{2}(n)+\frac{2484}{19} a_{2}\left(\frac{n}{3}\right)+22 a_{3}(n) \\
& -\frac{20952}{19} a_{3}\left(\frac{n}{3}\right)+\frac{152964}{19} a_{5}(n)+\frac{450048}{19} a_{5}\left(\frac{n}{2}\right)-98 a_{6}(n)+\frac{21440}{19} a_{6}\left(\frac{n}{2}\right) \\
& -\frac{90784}{19} a_{7}(n)+\frac{46976}{19} a_{8}(n)+\frac{156544}{19} a_{9}(n) \text {, } \\
& N\left(1^{4}, 2^{6}, 3^{4}, 6^{2} ; n\right)=\frac{61}{6691200} \sigma_{7}(n)-\frac{61}{6691200} \sigma_{7}\left(\frac{n}{2}\right)-\frac{567}{2230400} \sigma_{7}\left(\frac{n}{3}\right)+\frac{61}{418200} \sigma_{7}\left(\frac{n}{4}\right) \\
& +\frac{567}{2230400} \sigma_{7}\left(\frac{n}{6}\right)-\frac{1952}{52275} \sigma_{7}\left(\frac{n}{8}\right)-\frac{567}{139400} \sigma_{7}\left(\frac{n}{12}\right)+\frac{18144}{17425} \sigma_{7}\left(\frac{n}{24}\right)-\frac{108046753}{2616300} a_{1}(n) \\
& -\frac{78400}{513} a_{1}\left(\frac{n}{2}\right)+\frac{24414957}{32300} a_{1}\left(\frac{n}{3}\right)+\frac{378127168}{654075} a_{1}\left(\frac{n}{4}\right)+\frac{259560}{19} a_{1}\left(\frac{n}{6}\right)+\frac{644246208}{8075} a_{1}\left(\frac{n}{12}\right) \\
& -\frac{3270757}{350550} \Delta_{3,8}(n)-\frac{63062191}{175275} \Delta_{3,8}\left(\frac{n}{2}\right)+\frac{4082297984}{175275} \Delta_{3,8}\left(\frac{n}{4}\right)-\frac{1026540416}{175275} \Delta_{3,8}\left(\frac{n}{8}\right) \\
& +\frac{389548}{7695} a_{4}(n)-\frac{111104}{513} a_{4}\left(\frac{n}{2}\right)-\frac{5467648}{405} a_{4}\left(\frac{n}{4}\right)-20 a_{2}(n)+\frac{13068}{19} a_{2}\left(\frac{n}{3}\right)+28 a_{3}(n) \\
& -\frac{50004}{19} a_{3}\left(\frac{n}{3}\right)+\frac{295560}{19} a_{5}(n)+\frac{2815872}{19} a_{5}\left(\frac{n}{2}\right)-132 a_{6}(n)+\frac{108352}{19} a_{6}\left(\frac{n}{2}\right) \\
& -\frac{129536}{19} a_{7}(n)+\frac{148480}{19} a_{8}(n)+\frac{160256}{19} a_{9}(n) \text {, }
\end{aligned}
$$




$$
\begin{aligned}
& N\left(1^{4}, 2^{6}, 3^{6} ; n\right)=\frac{61}{3345600} \sigma_{7}(n)-\frac{61}{3345600} \sigma_{7}\left(\frac{n}{2}\right)-\frac{567}{1115200} \sigma_{7}\left(\frac{n}{3}\right)+\frac{61}{418200} \sigma_{7}\left(\frac{n}{4}\right) \\
& +\frac{567}{1115200} \sigma_{7}\left(\frac{n}{6}\right)-\frac{1952}{52275} \sigma_{7}\left(\frac{n}{8}\right)-\frac{567}{139400} \sigma_{7}\left(\frac{n}{12}\right)+\frac{18144}{17425} \sigma_{7}\left(\frac{n}{24}\right)-\frac{40312834}{654075} a_{1}(n) \\
& -\frac{22804}{171} a_{1}\left(\frac{n}{2}\right)+\frac{9671121}{8075} a_{1}\left(\frac{n}{3}\right)+\frac{135677248}{654075} a_{1}\left(\frac{n}{4}\right)+\frac{383004}{19} a_{1}\left(\frac{n}{6}\right)+\frac{980013888}{8075} a_{1}\left(\frac{n}{12}\right) \\
& -\frac{5182159}{350550} \Delta_{3,8}(n)-\frac{101675467}{175275} \Delta_{3,8}\left(\frac{n}{2}\right)+\frac{7659812024}{175275} \Delta_{3,8}\left(\frac{n}{4}\right)+\frac{651140224}{175275} \Delta_{3,8}\left(\frac{n}{8}\right) \\
& +\frac{587956}{7695} a_{4}(n)-\frac{60896}{171} a_{4}\left(\frac{n}{2}\right)-\frac{11365888}{405} a_{4}\left(\frac{n}{4}\right)-32 a_{2}(n)+\frac{17928}{19} a_{2}\left(\frac{n}{3}\right)+40 a_{3}(n) \\
& -\frac{73332}{19} a_{3}\left(\frac{n}{3}\right)+\frac{462528}{19} a_{5}(n)+\frac{5777664}{19} a_{5}\left(\frac{n}{2}\right)-200 a_{6}(n)+\frac{215488}{19} a_{6}\left(\frac{n}{2}\right) \\
& -\frac{172736}{19} a_{7}(n)+\frac{284800}{19} a_{8}(n)+\frac{142976}{19} a_{9}(n) \text {, } \\
& N\left(1^{4}, 2^{8}, 6^{4} ; n\right)=\frac{91}{13382400} \sigma_{7}(n)-\frac{91}{13382400} \sigma_{7}\left(\frac{n}{2}\right)+\frac{243}{4460800} \sigma_{7}\left(\frac{n}{3}\right)-\frac{91}{209100} \sigma_{7}\left(\frac{n}{4}\right) \\
& -\frac{243}{4460800} \sigma_{7}\left(\frac{n}{6}\right)+\frac{5824}{52275} \sigma_{7}\left(\frac{n}{8}\right)-\frac{243}{69700} \sigma_{7}\left(\frac{n}{12}\right)+\frac{15552}{17425} \sigma_{7}\left(\frac{n}{24}\right)-\frac{44628883}{5232600} a_{1}(n) \\
& -\frac{200858}{855} a_{1}\left(\frac{n}{2}\right)-\frac{6170373}{64600} a_{1}\left(\frac{n}{3}\right)+\frac{986075264}{654075} a_{1}\left(\frac{n}{4}\right)+\frac{28818}{95} a_{1}\left(\frac{n}{6}\right)+\frac{274425984}{8075} a_{1}\left(\frac{n}{12}\right) \\
& +\frac{826523}{701100} \Delta_{3,8}(n)-\frac{18343771}{350550} \Delta_{3,8}\left(\frac{n}{2}\right)-\frac{2555107928}{175275} \Delta_{3,8}\left(\frac{n}{4}\right)-\frac{4839290368}{175275} \Delta_{3,8}\left(\frac{n}{8}\right) \\
& +\frac{56534}{7695} a_{4}(n)+\frac{22240}{171} a_{4}\left(\frac{n}{2}\right)+\frac{5564416}{405} a_{4}\left(\frac{n}{4}\right)-4 a_{2}(n)-\frac{3834}{19} a_{2}\left(\frac{n}{3}\right)+12 a_{3}(n) \\
& +\frac{3834}{19} a_{3}\left(\frac{n}{3}\right)+\frac{1980}{19} a_{5}(n)-\frac{2750208}{19} a_{5}\left(\frac{n}{2}\right)-38 a_{6}(n)-\frac{92544}{19} a_{6}\left(\frac{n}{2}\right)-\frac{56512}{19} a_{7}(n) \\
& -\frac{90112}{19} a_{8}(n)+\frac{189952}{19} a_{9}(n) \text {, } \\
& N\left(1^{4}, 2^{8}, 3^{2}, 6^{2} ; n\right)=\frac{91}{6691200} \sigma_{7}(n)-\frac{91}{6691200} \sigma_{7}\left(\frac{n}{2}\right)+\frac{243}{2230400} \sigma_{7}\left(\frac{n}{3}\right)-\frac{91}{209100} \sigma_{7}\left(\frac{n}{4}\right) \\
& -\frac{243}{2230400} \sigma_{7}\left(\frac{n}{6}\right)+\frac{5824}{52275} \sigma_{7}\left(\frac{n}{8}\right)-\frac{243}{69700} \sigma_{7}\left(\frac{n}{12}\right)+\frac{15552}{17425} \sigma_{7}\left(\frac{n}{24}\right)+\frac{76578907}{2616300} a_{1}(n) \\
& -\frac{118456}{855} a_{1}\left(\frac{n}{2}\right)-\frac{39152583}{32300} a_{1}\left(\frac{n}{3}\right)+\frac{1009706624}{654075} a_{1}\left(\frac{n}{4}\right)-\frac{1873224}{95} a_{1}\left(\frac{n}{6}\right)+\frac{276188544}{8075} a_{1}\left(\frac{n}{12}\right) \\
& +\frac{2622854}{175275} \Delta_{3,8}(n)-\frac{11471966}{175275} \Delta_{3,8}\left(\frac{n}{2}\right)-\frac{7336728008}{175275} \Delta_{3,8}\left(\frac{n}{4}\right)-\frac{5612005888}{175275} \Delta_{3,8}\left(\frac{n}{8}\right) \\
& -\frac{340432}{7695} a_{4}(n)+\frac{130976}{171} a_{4}\left(\frac{n}{2}\right)+\frac{11769856}{405} a_{4}\left(\frac{n}{4}\right)-\frac{49518}{19} a_{2}\left(\frac{n}{3}\right)+8 a_{3}(n) \\
& +\frac{104922}{19} a_{3}\left(\frac{n}{3}\right)-\frac{380232}{19} a_{5}(n)-\frac{6019584}{19} a_{5}\left(\frac{n}{2}\right)-4 a_{6}(n)-\frac{218752}{19} a_{6}\left(\frac{n}{2}\right) \\
& +\frac{97856}{19} a_{7}(n)-\frac{226048}{19} a_{8}(n)-\frac{19712}{19} a_{9}(n) \text {, }
\end{aligned}
$$




$$
\begin{aligned}
& N\left(1^{4}, 2^{8}, 3^{4} ; n\right)=\frac{91}{3345600} \sigma_{7}(n)-\frac{91}{3345600} \sigma_{7}\left(\frac{n}{2}\right)+\frac{243}{1115200} \sigma_{7}\left(\frac{n}{3}\right)-\frac{91}{209100} \sigma_{7}\left(\frac{n}{4}\right) \\
& -\frac{243}{1115200} \sigma_{7}\left(\frac{n}{6}\right)+\frac{5824}{52275} \sigma_{7}\left(\frac{n}{8}\right)-\frac{243}{69700} \sigma_{7}\left(\frac{n}{12}\right)+\frac{15552}{17425} \sigma_{7}\left(\frac{n}{24}\right)+\frac{29562839}{436050} a_{1}(n) \\
& -\frac{103352}{855} a_{1}\left(\frac{n}{2}\right)-\frac{38636973}{16150} a_{1}\left(\frac{n}{3}\right)+\frac{483913088}{218025} a_{1}\left(\frac{n}{4}\right)-\frac{3543048}{95} a_{1}\left(\frac{n}{6}\right)+\frac{251904384}{8075} a_{1}\left(\frac{n}{12}\right) \\
& +\frac{1725541}{58425} \Delta_{3,8}(n)+\frac{5193086}{58425} \Delta_{3,8}\left(\frac{n}{2}\right)-\frac{4604556736}{58425} \Delta_{3,8}\left(\frac{n}{4}\right)-\frac{2863782656}{58425} \Delta_{3,8}\left(\frac{n}{8}\right) \\
& -\frac{249688}{2565} a_{4}(n)+\frac{224512}{171} a_{4}\left(\frac{n}{2}\right)+\frac{7263232}{135} a_{4}\left(\frac{n}{4}\right)+8 a_{2}(n)-\frac{90072}{19} a_{2}\left(\frac{n}{3}\right)+\frac{200880}{19} a_{3}\left(\frac{n}{3}\right) \\
& -\frac{759024}{19} a_{5}(n)-\frac{11185920}{19} a_{5}\left(\frac{n}{2}\right)+56 a_{6}(n)-\frac{403328}{19} a_{6}\left(\frac{n}{2}\right)+\frac{224256}{19} a_{7}(n) \\
& -\frac{430080}{19} a_{8}(n)-\frac{132096}{19} a_{9}(n) \text {, } \\
& N\left(1^{4}, 2^{10}, 6^{2} ; n\right)=\frac{547}{26764800} \sigma_{7}(n)-\frac{547}{26764800} \sigma_{7}\left(\frac{n}{2}\right)-\frac{729}{8921600} \sigma_{7}\left(\frac{n}{3}\right)+\frac{547}{418200} \sigma_{7}\left(\frac{n}{4}\right) \\
& +\frac{729}{8921600} \sigma_{7}\left(\frac{n}{6}\right)-\frac{17504}{52275} \sigma_{7}\left(\frac{n}{8}\right)-\frac{729}{139400} \sigma_{7}\left(\frac{n}{12}\right)+\frac{23328}{17425} \sigma_{7}\left(\frac{n}{24}\right)-\frac{6141173}{387600} a_{1}(n) \\
& -\frac{483293}{2565} a_{1}\left(\frac{n}{2}\right)-\frac{4613301}{129200} a_{1}\left(\frac{n}{3}\right)+\frac{246167872}{218025} a_{1}\left(\frac{n}{4}\right)-\frac{227799}{95} a_{1}\left(\frac{n}{6}\right)+\frac{476853696}{8075} a_{1}\left(\frac{n}{12}\right) \\
& +\frac{206267}{467400} \Delta_{3,8}(n)-\frac{85370239}{233700} \Delta_{3,8}\left(\frac{n}{2}\right)-\frac{168841804}{58425} \Delta_{3,8}\left(\frac{n}{4}\right)-\frac{1091166464}{58425} \Delta_{3,8}\left(\frac{n}{8}\right) \\
& +\frac{4387}{285} a_{4}(n)+\frac{154480}{513} a_{4}\left(\frac{n}{2}\right)+\frac{416768}{135} a_{4}\left(\frac{n}{4}\right)-13 a_{2}(n)-\frac{15471}{19} a_{2}\left(\frac{n}{3}\right)+21 a_{3}(n) \\
& +\frac{15471}{19} a_{3}\left(\frac{n}{3}\right)+\frac{19926}{19} a_{5}(n)-\frac{593280}{19} a_{5}\left(\frac{n}{2}\right)-107 a_{6}(n)-\frac{24768}{19} a_{6}\left(\frac{n}{2}\right) \\
& -\frac{42144}{19} a_{7}(n)+\frac{768}{19} a_{8}(n)+\frac{100608}{19} a_{9}(n), \\
& N\left(1^{4}, 2^{10}, 3^{2} ; n\right)=\frac{547}{13382400} \sigma_{7}(n)-\frac{547}{13382400} \sigma_{7}\left(\frac{n}{2}\right)-\frac{729}{4460800} \sigma_{7}\left(\frac{n}{3}\right)+\frac{547}{418200} \sigma_{7}\left(\frac{n}{4}\right) \\
& +\frac{729}{4460800} \sigma_{7}\left(\frac{n}{6}\right)-\frac{17504}{52275} \sigma_{7}\left(\frac{n}{8}\right)-\frac{729}{139400} \sigma_{7}\left(\frac{n}{12}\right)+\frac{23328}{17425} \sigma_{7}\left(\frac{n}{24}\right)-\frac{70134799}{581400} a_{1}(n) \\
& -\frac{116048}{285} a_{1}\left(\frac{n}{2}\right)+\frac{213466779}{64600} a_{1}\left(\frac{n}{3}\right)+\frac{12248128}{24225} a_{1}\left(\frac{n}{4}\right)+\frac{5382504}{95} a_{1}\left(\frac{n}{6}\right)+\frac{65295936}{8075} a_{1}\left(\frac{n}{12}\right) \\
& -\frac{9533693}{233700} \Delta_{3,8}(n)-\frac{21391379}{116850} \Delta_{3,8}\left(\frac{n}{2}\right)+\frac{4641668516}{58425} \Delta_{3,8}\left(\frac{n}{4}\right)+\frac{181893376}{58425} \Delta_{3,8}\left(\frac{n}{8}\right) \\
& +\frac{138002}{855} a_{4}(n)-\frac{96592}{57} a_{4}\left(\frac{n}{2}\right)-\frac{662528}{15} a_{4}\left(\frac{n}{4}\right)-18 a_{2}(n)+\frac{129600}{19} a_{2}\left(\frac{n}{3}\right)+26 a_{3}(n) \\
& -\frac{295812}{19} a_{3}\left(\frac{n}{3}\right)+\frac{1145340}{19} a_{5}(n)+\frac{9508608}{19} a_{5}\left(\frac{n}{2}\right)-158 a_{6}(n)+\frac{360512}{19} a_{6}\left(\frac{n}{2}\right) \\
& -\frac{486240}{19} a_{7}(n)+\frac{398208}{19} a_{8}(n)+\frac{684672}{19} a_{9}(n) \text {, }
\end{aligned}
$$




$$
\begin{aligned}
& N\left(1^{4}, 2^{12} ; n\right)=\frac{1}{16320} \sigma_{7}(n)-\frac{1}{16320} \sigma_{7}\left(\frac{n}{2}\right)-\frac{1}{255} \sigma_{7}\left(\frac{n}{4}\right)+\frac{256}{255} \sigma_{7}\left(\frac{n}{8}\right) \\
& -\frac{1}{34} a_{1}(n)-12 a_{1}\left(\frac{n}{2}\right)-\frac{512}{17} a_{1}\left(\frac{n}{4}\right)-2 a_{2}(n)+10 a_{3}(n), \\
& N\left(1^{5}, 2,3,6^{9} ; n\right)=\frac{13}{26764800} \sigma_{7}(n)-\frac{13}{26764800} \sigma_{7}\left(\frac{n}{2}\right)+\frac{1089}{8921600} \sigma_{7}\left(\frac{n}{3}\right) \\
& -\frac{13}{836400} \sigma_{7}\left(\frac{n}{4}\right)-\frac{1089}{8921600} \sigma_{7}\left(\frac{n}{6}\right)+\frac{208}{52275} \sigma_{7}\left(\frac{n}{8}\right)-\frac{1089}{278800} \sigma_{7}\left(\frac{n}{12}\right) \\
& +\frac{17424}{17425} \sigma_{7}\left(\frac{n}{24}\right)-\frac{121539019}{10465200} a_{1}(n)-\frac{674714}{2565} a_{1}\left(\frac{n}{2}\right)-\frac{18379989}{129200} a_{1}\left(\frac{n}{3}\right) \\
& +\frac{1097063008}{654075} a_{1}\left(\frac{n}{4}\right)-\frac{142002}{95} a_{1}\left(\frac{n}{6}\right)+\frac{372073248}{8075} a_{1}\left(\frac{n}{12}\right) \\
& +\frac{307708}{175275} \Delta_{3,8}(n)-\frac{71743979}{350550} \Delta_{3,8}\left(\frac{n}{2}\right)-\frac{2468626906}{175275} \Delta_{3,8}\left(\frac{n}{4}\right) \\
& -\frac{5269172096}{175275} \Delta_{3,8}\left(\frac{n}{8}\right)+\frac{75856}{7695} a_{4}(n)+\frac{135256}{513} a_{4}\left(\frac{n}{2}\right) \\
& +\frac{5222912}{405} a_{4}\left(\frac{n}{4}\right)-9 a_{2}(n)-\frac{9963}{19} a_{2}\left(\frac{n}{3}\right)+19 a_{3}(n) \\
& +\frac{10989}{19} a_{3}\left(\frac{n}{3}\right)-\frac{8310}{19} a_{5}(n)-\frac{2567040}{19} a_{5}\left(\frac{n}{2}\right)-75 a_{6}(n) \\
& -\frac{89760}{19} a_{6}\left(\frac{n}{2}\right)-\frac{61616}{19} a_{7}(n)-\frac{79424}{19} a_{8}(n)+\frac{186816}{19} a_{9}(n), \\
& N\left(1^{5}, 2,3^{3}, 6^{7} ; n\right)=\frac{13}{13382400} \sigma_{7}(n)-\frac{13}{13382400} \sigma_{7}\left(\frac{n}{2}\right)+\frac{1089}{4460800} \sigma_{7}\left(\frac{n}{3}\right) \\
& -\frac{13}{836400} \sigma_{7}\left(\frac{n}{4}\right)-\frac{1089}{4460800} \sigma_{7}\left(\frac{n}{6}\right)+\frac{208}{52275} \sigma_{7}\left(\frac{n}{8}\right)-\frac{1089}{278800} \sigma_{7}\left(\frac{n}{12}\right) \\
& +\frac{17424}{17425} \sigma_{7}\left(\frac{n}{24}\right)-\frac{17033683}{1744200} a_{1}(n)-\frac{136846}{513} a_{1}\left(\frac{n}{2}\right)-\frac{12276819}{64600} a_{1}\left(\frac{n}{3}\right) \\
& +\frac{13806048}{8075} a_{1}\left(\frac{n}{4}\right)-\frac{45738}{19} a_{1}\left(\frac{n}{6}\right)+\frac{343333728}{8075} a_{1}\left(\frac{n}{12}\right)+\frac{547973}{233700} \Delta_{3,8}(n) \\
& -\frac{22452301}{116850} \Delta_{3,8}\left(\frac{n}{2}\right)-\frac{933861952}{58425} \Delta_{3,8}\left(\frac{n}{4}\right)-\frac{601838784}{19475} \Delta_{3,8}\left(\frac{n}{8}\right) \\
& +\frac{19034}{2565} a_{4}(n)+\frac{146944}{513} a_{4}\left(\frac{n}{2}\right)+\frac{70912}{5} a_{4}\left(\frac{n}{4}\right)-8 a_{2}(n) \\
& -\frac{10962}{19} a_{2}\left(\frac{n}{3}\right)+18 a_{3}(n)+\frac{14040}{19} a_{3}\left(\frac{n}{3}\right)-\frac{24756}{19} a_{5}(n) \\
& -\frac{2828928}{19} a_{5}\left(\frac{n}{2}\right)-70 a_{6}(n)-\frac{99744}{19} a_{6}\left(\frac{n}{2}\right)-\frac{56384}{19} a_{7}(n) \\
& -\frac{91840}{19} a_{8}(n)+\frac{186112}{19} a_{9}(n) \text {, }
\end{aligned}
$$




$$
\begin{aligned}
& N\left(1^{5}, 2,3^{5}, 6^{5} ; n\right)=\frac{13}{6691200} \sigma_{7}(n)-\frac{13}{6691200} \sigma_{7}\left(\frac{n}{2}\right)+\frac{1089}{2230400} \sigma_{7}\left(\frac{n}{3}\right)-\frac{13}{836400} \sigma_{7}\left(\frac{n}{4}\right) \\
& -\frac{1089}{2230400} \sigma_{7}\left(\frac{n}{6}\right)+\frac{208}{52275} \sigma_{7}\left(\frac{n}{8}\right)-\frac{1089}{278800} \sigma_{7}\left(\frac{n}{12}\right)+\frac{17424}{17425} \sigma_{7}\left(\frac{n}{24}\right)-\frac{9593687}{1308150} a_{1}(n) \\
& -\frac{26886}{95} a_{1}\left(\frac{n}{2}\right)-\frac{1995336}{8075} a_{1}\left(\frac{n}{3}\right)+\frac{1186431328}{654075} a_{1}\left(\frac{n}{4}\right)-\frac{337806}{95} a_{1}\left(\frac{n}{6}\right)+\frac{270579168}{8075} a_{1}\left(\frac{n}{12}\right) \\
& +\frac{2136763}{701100} \Delta_{3,8}(n)-\frac{56274041}{350550} \Delta_{3,8}\left(\frac{n}{2}\right)-\frac{3398146516}{175275} \Delta_{3,8}\left(\frac{n}{4}\right)-\frac{5819779136}{175275} \Delta_{3,8}\left(\frac{n}{8}\right) \\
& +\frac{32974}{7695} a_{4}(n)+\frac{17648}{57} a_{4}\left(\frac{n}{2}\right)+\frac{6770432}{405} a_{4}\left(\frac{n}{4}\right)-6 a_{2}(n)-\frac{11016}{19} a_{2}\left(\frac{n}{3}\right)+16 a_{3}(n) \\
& +\frac{16146}{19} a_{3}\left(\frac{n}{3}\right)-\frac{45216}{19} a_{5}(n)-\frac{3333888}{19} a_{5}\left(\frac{n}{2}\right)-60 a_{6}(n)-\frac{118688}{19} a_{6}\left(\frac{n}{2}\right)-\frac{53472}{19} a_{7}(n) \\
& -\frac{115648}{19} a_{8}(n)+\frac{198464}{19} a_{9}(n) \\
& N\left(1^{5}, 2,3^{7}, 6^{3} ; n\right)=\frac{13}{3345600} \sigma_{7}(n)-\frac{13}{3345600} \sigma_{7}\left(\frac{n}{2}\right)+\frac{1089}{1115200} \sigma_{7}\left(\frac{n}{3}\right)-\frac{13}{836400} \sigma_{7}\left(\frac{n}{4}\right) \\
& -\frac{1089}{1115200} \sigma_{7}\left(\frac{n}{6}\right)+\frac{208}{52275} \sigma_{7}\left(\frac{n}{8}\right)-\frac{1089}{278800} \sigma_{7}\left(\frac{n}{12}\right)+\frac{17424}{17425} \sigma_{7}\left(\frac{n}{24}\right)-\frac{2355172}{654075} a_{1}(n) \\
& -\frac{2455216}{7695} a_{1}\left(\frac{n}{2}\right)-\frac{2589957}{8075} a_{1}\left(\frac{n}{3}\right)+\frac{441742496}{218025} a_{1}\left(\frac{n}{4}\right)-\frac{499356}{95} a_{1}\left(\frac{n}{6}\right)+\frac{111997728}{8075} a_{1}\left(\frac{n}{12}\right) \\
& +\frac{1386053}{350550} \Delta_{3,8}(n)-\frac{5509547}{58425} \Delta_{3,8}\left(\frac{n}{2}\right)-\frac{4443036436}{175275} \Delta_{3,8}\left(\frac{n}{4}\right)-\frac{735137984}{19475} \Delta_{3,8}\left(\frac{n}{8}\right) \\
& -\frac{2732}{7695} a_{4}(n)+\frac{514256}{1539} a_{4}\left(\frac{n}{2}\right)+\frac{2877184}{135} a_{4}\left(\frac{n}{4}\right)-2 a_{2}(n)-\frac{9342}{19} a_{2}\left(\frac{n}{3}\right)+12 a_{3}(n) \\
& +\frac{16524}{19} a_{3}\left(\frac{n}{3}\right)-\frac{73296}{19} a_{5}(n)-\frac{4238400}{19} a_{5}\left(\frac{n}{2}\right)-40 a_{6}(n)-\frac{152800}{19} a_{6}\left(\frac{n}{2}\right) \\
& -\frac{53760}{19} a_{7}(n)-\frac{159488}{19} a_{8}(n)+\frac{231424}{19} a_{9}(n) \text {, } \\
& N\left(1^{5}, 2,3^{9}, 6 ; n\right)=\frac{13}{1672800} \sigma_{7}(n)-\frac{13}{1672800} \sigma_{7}\left(\frac{n}{2}\right)+\frac{1089}{557600} \sigma_{7}\left(\frac{n}{3}\right)-\frac{13}{836400} \sigma_{7}\left(\frac{n}{4}\right) \\
& -\frac{1089}{557600} \sigma_{7}\left(\frac{n}{6}\right)+\frac{208}{52275} \sigma_{7}\left(\frac{n}{8}\right)-\frac{1089}{278800} \sigma_{7}\left(\frac{n}{12}\right)+\frac{17424}{17425} \sigma_{7}\left(\frac{n}{24}\right)+\frac{1733081}{654075} a_{1}(n) \\
& -\frac{3142276}{7695} a_{1}\left(\frac{n}{2}\right)-\frac{3462489}{8075} a_{1}\left(\frac{n}{3}\right)+\frac{182375392}{72675} a_{1}\left(\frac{n}{4}\right)-\frac{803376}{95} a_{1}\left(\frac{n}{6}\right)-\frac{231995232}{8075} a_{1}\left(\frac{n}{12}\right) \\
& +\frac{925828}{175275} \Delta_{3,8}(n)+\frac{2357566}{58425} \Delta_{3,8}\left(\frac{n}{2}\right)-\frac{6533293516}{175275} \Delta_{3,8}\left(\frac{n}{4}\right)-\frac{931360704}{19475} \Delta_{3,8}\left(\frac{n}{8}\right) \\
& -\frac{61064}{7695} a_{4}(n)+\frac{576176}{1539} a_{4}\left(\frac{n}{2}\right)+\frac{1380608}{45} a_{4}\left(\frac{n}{4}\right)+6 a_{2}(n)-\frac{4158}{19} a_{2}\left(\frac{n}{3}\right)+4 a_{3}(n) \\
& +\frac{13392}{19} a_{3}\left(\frac{n}{3}\right)-\frac{117360}{19} a_{5}(n)-\frac{6068160}{19} a_{5}\left(\frac{n}{2}\right)-\frac{222624}{19} a_{6}\left(\frac{n}{2}\right)-\frac{62144}{19} a_{7}(n) \\
& -\frac{249984}{19} a_{8}(n)+\frac{312704}{19} a_{9}(n) \text {, }
\end{aligned}
$$




$$
\begin{aligned}
& N\left(1^{5}, 2^{3}, 3,6^{7} ; n\right)=\frac{1}{652800} \sigma_{7}(n)-\frac{1}{652800} \sigma_{7}\left(\frac{n}{2}\right)-\frac{27}{217600} \sigma_{7}\left(\frac{n}{3}\right)+\frac{1}{20400} \sigma_{7}\left(\frac{n}{4}\right) \\
& +\frac{27}{217600} \sigma_{7}\left(\frac{n}{6}\right)-\frac{16}{1275} \sigma_{7}\left(\frac{n}{8}\right)-\frac{27}{6800} \sigma_{7}\left(\frac{n}{12}\right)+\frac{432}{425} \sigma_{7}\left(\frac{n}{24}\right)-\frac{58786321}{3488400} a_{1}(n) \\
& -\frac{564322}{2565} a_{1}\left(\frac{n}{2}\right)-\frac{1172853}{129200} a_{1}\left(\frac{n}{3}\right)+\frac{100838176}{72675} a_{1}\left(\frac{n}{4}\right)+\frac{84294}{95} a_{1}\left(\frac{n}{6}\right)+\frac{537892704}{8075} a_{1}\left(\frac{n}{12}\right) \\
& +\frac{643}{5700} \Delta_{3,8}(n)-\frac{121931}{475} \Delta_{3,8}\left(\frac{n}{2}\right)-\frac{8331206}{1425} \Delta_{3,8}\left(\frac{n}{4}\right)-\frac{34026496}{1425} \Delta_{3,8}\left(\frac{n}{8}\right) \\
& +\frac{42934}{2565} a_{4}(n)+\frac{98360}{513} a_{4}\left(\frac{n}{2}\right)+\frac{303104}{45} a_{4}\left(\frac{n}{4}\right)-13 a_{2}(n)-\frac{10287}{19} a_{2}\left(\frac{n}{3}\right)+23 a_{3}(n) \\
& +\frac{7209}{19} a_{3}\left(\frac{n}{3}\right)+\frac{42210}{19} a_{5}(n)-\frac{1343232}{19} a_{5}\left(\frac{n}{2}\right)-91 a_{6}(n)-\frac{44128}{19} a_{6}\left(\frac{n}{2}\right)-\frac{63600}{19} a_{7}(n) \\
& -\frac{22848}{19} a_{8}(n)+\frac{151488}{19} a_{9}(n) \text {, } \\
& N\left(1^{5}, 2^{3}, 3^{3}, 6^{5} ; n\right)=\frac{1}{326400} \sigma_{7}(n)-\frac{1}{326400} \sigma_{7}\left(\frac{n}{2}\right)-\frac{27}{108800} \sigma_{7}\left(\frac{n}{3}\right)+\frac{1}{20400} \sigma_{7}\left(\frac{n}{4}\right) \\
& +\frac{27}{108800} \sigma_{7}\left(\frac{n}{6}\right)-\frac{16}{1275} \sigma_{7}\left(\frac{n}{8}\right)-\frac{27}{6800} \sigma_{7}\left(\frac{n}{12}\right)+\frac{432}{425} \sigma_{7}\left(\frac{n}{24}\right)-\frac{13011737}{581400} a_{1}(n) \\
& -\frac{102838}{513} a_{1}\left(\frac{n}{2}\right)+\frac{9140877}{64600} a_{1}\left(\frac{n}{3}\right)+\frac{266403808}{218025} a_{1}\left(\frac{n}{4}\right)+\frac{71334}{19} a_{1}\left(\frac{n}{6}\right)+\frac{635274144}{8075} a_{1}\left(\frac{n}{12}\right) \\
& -\frac{9949}{5700} \Delta_{3,8}(n)-\frac{268629}{950} \Delta_{3,8}\left(\frac{n}{2}\right)+\frac{346648}{475} \Delta_{3,8}\left(\frac{n}{4}\right)-\frac{28632256}{1425} \Delta_{3,8}\left(\frac{n}{8}\right) \\
& +\frac{20626}{855} a_{4}(n)+\frac{52960}{513} a_{4}\left(\frac{n}{2}\right)+\frac{296192}{135} a_{4}\left(\frac{n}{4}\right)-16 a_{2}(n)-\frac{7290}{19} a_{2}\left(\frac{n}{3}\right)+26 a_{3}(n) \\
& -\frac{1944}{19} a_{3}\left(\frac{n}{3}\right)+\frac{97020}{19} a_{5}(n)-\frac{426240}{19} a_{5}\left(\frac{n}{2}\right)-102 a_{6}(n)-\frac{9312}{19} a_{6}\left(\frac{n}{2}\right)-\frac{75648}{19} a_{7}(n) \\
& +\frac{19264}{19} a_{8}(n)+\frac{146304}{19} a_{9}(n) \text {, } \\
& N\left(1^{5}, 2^{3}, 3^{5}, 6^{3} ; n\right)=\frac{1}{163200} \sigma_{7}(n)-\frac{1}{163200} \sigma_{7}\left(\frac{n}{2}\right)-\frac{27}{54400} \sigma_{7}\left(\frac{n}{3}\right)+\frac{1}{20400} \sigma_{7}\left(\frac{n}{4}\right) \\
& +\frac{27}{54400} \sigma_{7}\left(\frac{n}{6}\right)-\frac{16}{1275} \sigma_{7}\left(\frac{n}{8}\right)-\frac{27}{6800} \sigma_{7}\left(\frac{n}{12}\right)+\frac{432}{425} \sigma_{7}\left(\frac{n}{24}\right)-\frac{1425227}{48450} a_{1}(n) \\
& -\frac{24046}{171} a_{1}\left(\frac{n}{2}\right)+\frac{2597913}{8075} a_{1}\left(\frac{n}{3}\right)+\frac{20375392}{24225} a_{1}\left(\frac{n}{4}\right)+\frac{139266}{19} a_{1}\left(\frac{n}{6}\right)+\frac{864700704}{8075} a_{1}\left(\frac{n}{12}\right) \\
& -\frac{7541}{1900} \Delta_{3,8}(n)-\frac{1008899}{2850} \Delta_{3,8}\left(\frac{n}{2}\right)+\frac{5624868}{475} \Delta_{3,8}\left(\frac{n}{4}\right)-\frac{16997696}{1425} \Delta_{3,8}\left(\frac{n}{8}\right) \\
& +\frac{9514}{285} a_{4}(n)+\frac{496}{171} a_{4}\left(\frac{n}{2}\right)-\frac{30464}{5} a_{4}\left(\frac{n}{4}\right)-22 a_{2}(n)-\frac{7128}{19} a_{2}\left(\frac{n}{3}\right)+32 a_{3}(n) \\
& -\frac{8262}{19} a_{3}\left(\frac{n}{3}\right)+\frac{163872}{19} a_{5}(n)+\frac{1219968}{19} a_{5}\left(\frac{n}{2}\right)-124 a_{6}(n)+\frac{52384}{19} a_{6}\left(\frac{n}{2}\right) \\
& -\frac{80736}{19} a_{7}(n)+\frac{95552}{19} a_{8}(n)+\frac{101952}{19} a_{9}(n) \text {, }
\end{aligned}
$$




$$
\begin{aligned}
& N\left(1^{5}, 2^{3}, 3^{7}, 6 ; n\right)=\frac{1}{81600} \sigma_{7}(n)-\frac{1}{81600} \sigma_{7}\left(\frac{n}{2}\right)-\frac{27}{27200} \sigma_{7}\left(\frac{n}{3}\right)+\frac{1}{20400} \sigma_{7}\left(\frac{n}{4}\right) \\
& +\frac{27}{27200} \sigma_{7}\left(\frac{n}{6}\right)-\frac{16}{1275} \sigma_{7}\left(\frac{n}{8}\right)-\frac{27}{6800} \sigma_{7}\left(\frac{n}{12}\right)+\frac{432}{425} \sigma_{7}\left(\frac{n}{24}\right)-\frac{8770928}{218025} a_{1}(n) \\
& -\frac{76552}{2565} a_{1}\left(\frac{n}{2}\right)+\frac{4440771}{8075} a_{1}\left(\frac{n}{3}\right)+\frac{44582368}{218025} a_{1}\left(\frac{n}{4}\right)+\frac{1164564}{95} a_{1}\left(\frac{n}{6}\right)+\frac{1340445024}{8075} a_{1}\left(\frac{n}{12}\right) \\
& -\frac{19333}{2850} \Delta_{3,8}(n)-\frac{715339}{1425} \Delta_{3,8}\left(\frac{n}{2}\right)+\frac{42681964}{1425} \Delta_{3,8}\left(\frac{n}{4}\right)+\frac{809408}{475} \Delta_{3,8}\left(\frac{n}{8}\right) \\
& +\frac{120572}{2565} a_{4}(n)-\frac{60592}{513} a_{4}\left(\frac{n}{2}\right)-\frac{2683648}{135} a_{4}\left(\frac{n}{4}\right)-34 a_{2}(n)-\frac{12150}{19} a_{2}\left(\frac{n}{3}\right)+44 a_{3}(n) \\
& -\frac{9396}{19} a_{3}\left(\frac{n}{3}\right)+\frac{259056}{19} a_{5}(n)+\frac{3933504}{19} a_{5}\left(\frac{n}{2}\right)-168 a_{6}(n)+\frac{154720}{19} a_{6}\left(\frac{n}{2}\right) \\
& -\frac{79872}{19} a_{7}(n)+\frac{227072}{19} a_{8}(n)+\frac{3072}{19} a_{9}(n) \text {, } \\
& N\left(1^{5}, 2^{5}, 3,6^{5} ; n\right)=\frac{121}{26764800} \sigma_{7}(n)-\frac{121}{26764800} \sigma_{7}\left(\frac{n}{2}\right)+\frac{1053}{8921600} \sigma_{7}\left(\frac{n}{3}\right)-\frac{121}{836400} \sigma_{7}\left(\frac{n}{4}\right) \\
& -\frac{1053}{8921600} \sigma_{7}\left(\frac{n}{6}\right)+\frac{1936}{52275} \sigma_{7}\left(\frac{n}{8}\right)-\frac{1053}{278800} \sigma_{7}\left(\frac{n}{12}\right)+\frac{16848}{17425} \sigma_{7}\left(\frac{n}{24}\right)-\frac{52092343}{10465200} a_{1}(n) \\
& -\frac{721418}{2565} a_{1}\left(\frac{n}{2}\right)-\frac{40092633}{129200} a_{1}\left(\frac{n}{3}\right)+\frac{1193265376}{654075} a_{1}\left(\frac{n}{4}\right)-\frac{459954}{95} a_{1}\left(\frac{n}{6}\right)+\frac{247633056}{8075} a_{1}\left(\frac{n}{12}\right) \\
& +\frac{1342727}{350550} \Delta_{3,8}(n)-\frac{49507313}{350550} \Delta_{3,8}\left(\frac{n}{2}\right)-\frac{3774051682}{175275} \Delta_{3,8}\left(\frac{n}{4}\right)-\frac{5915760512}{175275} \Delta_{3,8}\left(\frac{n}{8}\right) \\
& +\frac{8812}{7695} a_{4}(n)+\frac{173368}{513} a_{4}\left(\frac{n}{2}\right)+\frac{7361024}{405} a_{4}\left(\frac{n}{4}\right)-5 a_{2}(n)-\frac{13743}{19} a_{2}\left(\frac{n}{3}\right)+15 a_{3}(n) \\
& +\frac{22977}{19} a_{3}\left(\frac{n}{3}\right)-\frac{65214}{19} a_{5}(n)-\frac{3637632}{19} a_{5}\left(\frac{n}{2}\right)-55 a_{6}(n)-\frac{129312}{19} a_{6}\left(\frac{n}{2}\right)-\frac{42608}{19} a_{7}(n) \\
& -\frac{126272}{19} a_{8}(n)+\frac{188096}{19} a_{9}(n) \\
& N\left(1^{5}, 2^{5}, 3,6^{5} ; n\right)=\frac{121}{13382400} \sigma_{7}(n)-\frac{121}{13382400} \sigma_{7}\left(\frac{n}{2}\right)+\frac{1053}{4460800} \sigma_{7}\left(\frac{n}{3}\right)-\frac{121}{836400} \sigma_{7}\left(\frac{n}{4}\right) \\
& -\frac{1053}{4460800} \sigma_{7}\left(\frac{n}{6}\right)+\frac{1936}{52275} \sigma_{7}\left(\frac{n}{8}\right)-\frac{1053}{278800} \sigma_{7}\left(\frac{n}{12}\right)+\frac{16848}{17425} \sigma_{7}\left(\frac{n}{24}\right)+\frac{49731707}{5232600} a_{1}(n) \\
& -\frac{757478}{2565} a_{1}\left(\frac{n}{2}\right)-\frac{46449783}{64600} a_{1}\left(\frac{n}{3}\right)+\frac{1378649696}{654075} a_{1}\left(\frac{n}{4}\right)-\frac{1146114}{95} a_{1}\left(\frac{n}{6}\right)+\frac{133898976}{8075} a_{1}\left(\frac{n}{12}\right) \\
& +\frac{6222683}{701100} \Delta_{3,8}(n)-\frac{30945811}{350550} \Delta_{3,8}\left(\frac{n}{2}\right)-\frac{6336018272}{175275} \Delta_{3,8}\left(\frac{n}{4}\right)-\frac{7135356352}{175275} \Delta_{3,8}\left(\frac{n}{8}\right) \\
& -\frac{141466}{7695} a_{4}(n)+\frac{287104}{513} a_{4}\left(\frac{n}{2}\right)+\frac{11286784}{405} a_{4}\left(\frac{n}{4}\right)-\frac{25866}{19} a_{2}\left(\frac{n}{3}\right)+10 a_{3}(n)+\frac{53568}{19} a_{3}\left(\frac{n}{3}\right) \\
& -\frac{208836}{19} a_{5}(n)-\frac{5631360}{19} a_{5}\left(\frac{n}{2}\right)-30 a_{6}(n)-\frac{204064}{19} a_{6}\left(\frac{n}{2}\right)+\frac{704}{19} a_{7}(n)-\frac{213184}{19} a_{8}(n) \\
& +\frac{166144}{19} a_{9}(n) \text {, }
\end{aligned}
$$




$$
\begin{aligned}
& N\left(1^{5}, 2^{5}, 3^{5}, 6 ; n\right)=\frac{121}{6691200} \sigma_{7}(n)-\frac{121}{6691200} \sigma_{7}\left(\frac{n}{2}\right)+\frac{1053}{2230400} \sigma_{7}\left(\frac{n}{3}\right)-\frac{121}{836400} \sigma_{7}\left(\frac{n}{4}\right) \\
& -\frac{1053}{2230400} \sigma_{7}\left(\frac{n}{6}\right)+\frac{1936}{52275} \sigma_{7}\left(\frac{n}{8}\right)-\frac{1053}{278800} \sigma_{7}\left(\frac{n}{12}\right)+\frac{16848}{17425} \sigma_{7}\left(\frac{n}{24}\right)+\frac{1857299}{68850} a_{1}(n) \\
& -\frac{16058}{45} a_{1}\left(\frac{n}{2}\right)-\frac{498978}{425} a_{1}\left(\frac{n}{3}\right)+\frac{92084384}{34425} a_{1}\left(\frac{n}{4}\right)-\frac{98082}{5} a_{1}\left(\frac{n}{6}\right)-\frac{9264096}{425} a_{1}\left(\frac{n}{12}\right) \\
& +\frac{534749}{36900} \Delta_{3,8}(n)+\frac{1296017}{18450} \Delta_{3,8}\left(\frac{n}{2}\right)-\frac{529705868}{9225} \Delta_{3,8}\left(\frac{n}{4}\right)-\frac{494723008}{9225} \Delta_{3,8}\left(\frac{n}{8}\right) \\
& -\frac{16798}{405} a_{4}(n)+\frac{6832}{9} a_{4}\left(\frac{n}{2}\right)+\frac{17515264}{405} a_{4}\left(\frac{n}{4}\right)+10 a_{2}(n)-1728 a_{2}\left(\frac{n}{3}\right)+4158 a_{3}\left(\frac{n}{3}\right) \\
& -19296 a_{5}(n)-460032 a_{5}\left(\frac{n}{2}\right)+20 a_{6}(n)-16736 a_{6}\left(\frac{n}{2}\right)+1760 a_{7}(n) \\
& -18496 a_{8}(n)+10432 a_{9}(n) \text {, } \\
& N\left(1^{5}, 2^{7}, 3,6^{3} ; n\right)=\frac{73}{5352960} \sigma_{7}(n)-\frac{73}{5352960} \sigma_{7}\left(\frac{n}{2}\right)-\frac{243}{1784320} \sigma_{7}\left(\frac{n}{3}\right)+\frac{73}{167280} \sigma_{7}\left(\frac{n}{4}\right) \\
& +\frac{243}{1784320} \sigma_{7}\left(\frac{n}{6}\right)-\frac{1168}{10455} \sigma_{7}\left(\frac{n}{8}\right)-\frac{243}{55760} \sigma_{7}\left(\frac{n}{12}\right)+\frac{3888}{3485} \sigma_{7}\left(\frac{n}{24}\right)-\frac{25506937}{697680} a_{1}(n) \\
& -\frac{221798}{855} a_{1}\left(\frac{n}{2}\right)+\frac{13425939}{25840} a_{1}\left(\frac{n}{3}\right)+\frac{11372000}{8721} a_{1}\left(\frac{n}{4}\right)+\frac{880038}{95} a_{1}\left(\frac{n}{6}\right)+\frac{23899104}{323} a_{1}\left(\frac{n}{12}\right) \\
& -\frac{299779}{46740} \Delta_{3,8}(n)-\frac{779056}{2337} \Delta_{3,8}\left(\frac{n}{2}\right)+\frac{108804346}{11685} \Delta_{3,8}\left(\frac{n}{4}\right)-\frac{47081984}{2337} \Delta_{3,8}\left(\frac{n}{8}\right) \\
& +\frac{22042}{513} a_{4}(n)-\frac{8504}{171} a_{4}\left(\frac{n}{2}\right)-\frac{71680}{27} a_{4}\left(\frac{n}{4}\right)-17 a_{2}(n)+\frac{6885}{19} a_{2}\left(\frac{n}{3}\right)+27 a_{3}(n) \\
& -\frac{34587}{19} a_{3}\left(\frac{n}{3}\right)+\frac{222858}{19} a_{5}(n)+\frac{633600}{19} a_{5}\left(\frac{n}{2}\right)-127 a_{6}(n)+\frac{28960}{19} a_{6}\left(\frac{n}{2}\right) \\
& -\frac{128688}{19} a_{7}(n)+\frac{62400}{19} a_{8}(n)+\frac{229056}{19} a_{9}(n) \text {, } \\
& N\left(1^{5}, 2^{7}, 3^{3}, 6 ; n\right)=\frac{73}{2676480} \sigma_{7}(n)-\frac{73}{2676480} \sigma_{7}\left(\frac{n}{2}\right)-\frac{243}{892160} \sigma_{7}\left(\frac{n}{3}\right)+\frac{73}{167280} \sigma_{7}\left(\frac{n}{4}\right) \\
& +\frac{243}{892160} \sigma_{7}\left(\frac{n}{6}\right)-\frac{1168}{10455} \sigma_{7}\left(\frac{n}{8}\right)-\frac{243}{55760} \sigma_{7}\left(\frac{n}{12}\right)+\frac{3888}{3485} \sigma_{7}\left(\frac{n}{24}\right)-\frac{15576509}{209304} a_{1}(n) \\
& -\frac{682526}{2565} a_{1}\left(\frac{n}{2}\right)+\frac{4343769}{2584} a_{1}\left(\frac{n}{3}\right)+\frac{17680672}{26163} a_{1}\left(\frac{n}{4}\right)+\frac{2738142}{95} a_{1}\left(\frac{n}{6}\right)+\frac{24613920}{323} a_{1}\left(\frac{n}{12}\right) \\
& -\frac{581963}{28044} \Delta_{3,8}(n)-\frac{28730461}{70110} \Delta_{3,8}\left(\frac{n}{2}\right)+\frac{1588937272}{35055} \Delta_{3,8}\left(\frac{n}{4}\right)-\frac{30001472}{7011} \Delta_{3,8}\left(\frac{n}{8}\right) \\
& +\frac{146450}{1539} a_{4}(n)-\frac{347936}{513} a_{4}\left(\frac{n}{2}\right)-\frac{2099456}{81} a_{4}\left(\frac{n}{4}\right)-24 a_{2}(n)+\frac{49518}{19} a_{2}\left(\frac{n}{3}\right)+34 a_{3}(n) \\
& -\frac{132624}{19} a_{3}\left(\frac{n}{3}\right)+\frac{612108}{19} a_{5}(n)+\frac{5494272}{19} a_{5}\left(\frac{n}{2}\right)-174 a_{6}(n)+\frac{208416}{19} a_{6}\left(\frac{n}{2}\right) \\
& -\frac{262016}{19} a_{7}(n)+\frac{258880}{19} a_{8}(n)+\frac{348032}{19} a_{9}(n) \text {, }
\end{aligned}
$$




$$
\begin{aligned}
& N\left(1^{5}, 2^{9}, 3,6 ; n\right)=\frac{1093}{26764800} \sigma_{7}(n)-\frac{1093}{26764800} \sigma_{7}\left(\frac{n}{2}\right)+\frac{729}{8921600} \sigma_{7}\left(\frac{n}{3}\right)-\frac{1093}{836400} \sigma_{7}\left(\frac{n}{4}\right) \\
& -\frac{729}{8921600} \sigma_{7}\left(\frac{n}{6}\right)+\frac{17488}{52275} \sigma_{7}\left(\frac{n}{8}\right)-\frac{729}{278800} \sigma_{7}\left(\frac{n}{12}\right)+\frac{11664}{17425} \sigma_{7}\left(\frac{n}{24}\right)+\frac{8440933}{183600} a_{1}(n) \\
& -\frac{9694}{135} a_{1}\left(\frac{n}{2}\right)-\frac{11843631}{6800} a_{1}\left(\frac{n}{3}\right)+\frac{16465504}{11475} a_{1}\left(\frac{n}{4}\right)-\frac{146502}{5} a_{1}\left(\frac{n}{6}\right)+\frac{21663072}{425} a_{1}\left(\frac{n}{12}\right) \\
& +\frac{66119}{3075} \Delta_{3,8}(n)-\frac{801727}{6150} \Delta_{3,8}\left(\frac{n}{2}\right)-\frac{160212218}{3075} \Delta_{3,8}\left(\frac{n}{4}\right)-\frac{97176448}{3075} \Delta_{3,8}\left(\frac{n}{8}\right) \\
& -\frac{9112}{135} a_{4}(n)+\frac{29576}{27} a_{4}\left(\frac{n}{2}\right)+\frac{4580864}{135} a_{4}\left(\frac{n}{4}\right)-a_{2}(n)-3969 a_{2}\left(\frac{n}{3}\right)+11 a_{3}(n) \\
& +8343 a_{3}\left(\frac{n}{3}\right)-29538 a_{5}(n)-374400 a_{5}\left(\frac{n}{2}\right)-3 a_{6}(n)-13792 a_{6}\left(\frac{n}{2}\right) \\
& +9456 a_{7}(n)-14016 a_{8}(n)-7872 a_{9}(n) \text {, } \\
& N\left(1^{6}, 6^{10} ; n\right)=\frac{7}{13382400} \sigma_{7}(n)-\frac{7}{13382400} \sigma_{7}\left(\frac{n}{2}\right)-\frac{549}{4460800} \sigma_{7}\left(\frac{n}{3}\right)+\frac{7}{418200} \sigma_{7}\left(\frac{n}{4}\right) \\
& +\frac{549}{4460800} \sigma_{7}\left(\frac{n}{6}\right)-\frac{224}{52275} \sigma_{7}\left(\frac{n}{8}\right)-\frac{549}{139400} \sigma_{7}\left(\frac{n}{12}\right)+\frac{17568}{17425} \sigma_{7}\left(\frac{n}{24}\right)-\frac{84566171}{5232600} a_{1}(n) \\
& -\frac{164912}{513} a_{1}\left(\frac{n}{2}\right)-\frac{8144001}{64600} a_{1}\left(\frac{n}{3}\right)+\frac{1308738496}{654075} a_{1}\left(\frac{n}{4}\right)-\frac{34632}{19} a_{1}\left(\frac{n}{6}\right)+\frac{421690176}{8075} a_{1}\left(\frac{n}{12}\right) \\
& +\frac{1091701}{701100} \Delta_{3,8}(n)-\frac{98746037}{350550} \Delta_{3,8}\left(\frac{n}{2}\right)-\frac{2713504852}{175275} \Delta_{3,8}\left(\frac{n}{4}\right)-\frac{6243690752}{175275} \Delta_{3,8}\left(\frac{n}{8}\right) \\
& +\frac{112378}{7695} a_{4}(n)+\frac{169520}{513} a_{4}\left(\frac{n}{2}\right)+\frac{5848064}{405} a_{4}\left(\frac{n}{4}\right)-12 a_{2}(n)-\frac{11610}{19} a_{2}\left(\frac{n}{3}\right)+24 a_{3}(n) \\
& +\frac{11610}{19} a_{3}\left(\frac{n}{3}\right)+\frac{4236}{19} a_{5}(n)-\frac{2854656}{19} a_{5}\left(\frac{n}{2}\right)-102 a_{6}(n)-\frac{100288}{19} a_{6}\left(\frac{n}{2}\right) \\
& -\frac{79200}{19} a_{7}(n)-\frac{82048}{19} a_{8}(n)+\frac{224896}{19} a_{9}(n) \text {, } \\
& N\left(1^{6}, 3^{2}, 6^{8} ; n\right)=\frac{7}{6691200} \sigma_{7}(n)-\frac{7}{6691200} \sigma_{7}\left(\frac{n}{2}\right)-\frac{549}{2230400} \sigma_{7}\left(\frac{n}{3}\right)+\frac{7}{418200} \sigma_{7}\left(\frac{n}{4}\right) \\
& +\frac{549}{2230400} \sigma_{7}\left(\frac{n}{6}\right)-\frac{224}{52275} \sigma_{7}\left(\frac{n}{8}\right)-\frac{549}{139400} \sigma_{7}\left(\frac{n}{12}\right)+\frac{17568}{17425} \sigma_{7}\left(\frac{n}{24}\right)-\frac{46483621}{2616300} a_{1}(n) \\
& -\frac{829868}{2565} a_{1}\left(\frac{n}{2}\right)-\frac{2857851}{32300} a_{1}\left(\frac{n}{3}\right)+\frac{1299795136}{654075} a_{1}\left(\frac{n}{4}\right)-\frac{77364}{95} a_{1}\left(\frac{n}{6}\right)+\frac{481617216}{8075} a_{1}\left(\frac{n}{12}\right) \\
& +\frac{191713}{175275} \Delta_{3,8}(n)-\frac{52202632}{175275} \Delta_{3,8}\left(\frac{n}{2}\right)-\frac{2499103552}{175275} \Delta_{3,8}\left(\frac{n}{4}\right)-\frac{6169615232}{175275} \Delta_{3,8}\left(\frac{n}{8}\right) \\
& +\frac{128296}{7695} a_{4}(n)+\frac{163072}{513} a_{4}\left(\frac{n}{2}\right)+\frac{5517824}{405} a_{4}\left(\frac{n}{4}\right)-12 a_{2}(n)-\frac{10476}{19} a_{2}\left(\frac{n}{3}\right)+24 a_{3}(n) \\
& +\frac{8424}{19} a_{3}\left(\frac{n}{3}\right)+\frac{16200}{19} a_{5}(n)-\frac{2698368}{19} a_{5}\left(\frac{n}{2}\right)-108 a_{6}(n)-\frac{94016}{19} a_{6}\left(\frac{n}{2}\right)-\frac{83200}{19} a_{7}(n) \\
& -\frac{75776}{19} a_{8}(n)+\frac{229376}{19} a_{9}(n) \text {, }
\end{aligned}
$$




$$
\begin{aligned}
& N\left(1^{6}, 3^{4}, 6^{6} ; n\right)=\frac{7}{3345600} \sigma_{7}(n)-\frac{7}{3345600} \sigma_{7}\left(\frac{n}{2}\right)-\frac{549}{1115200} \sigma_{7}\left(\frac{n}{3}\right)+\frac{7}{418200} \sigma_{7}\left(\frac{n}{4}\right) \\
& +\frac{549}{1115200} \sigma_{7}\left(\frac{n}{6}\right)-\frac{224}{52275} \sigma_{7}\left(\frac{n}{8}\right)-\frac{549}{139400} \sigma_{7}\left(\frac{n}{12}\right)+\frac{17568}{17425} \sigma_{7}\left(\frac{n}{24}\right)-\frac{12869728}{654075} a_{1}(n) \\
& -\frac{2552108}{7695} a_{1}\left(\frac{n}{2}\right)-\frac{449343}{8075} a_{1}\left(\frac{n}{3}\right)+\frac{1286108096}{654075} a_{1}\left(\frac{n}{4}\right)-\frac{15228}{95} a_{1}\left(\frac{n}{6}\right)+\frac{471139776}{8075} a_{1}\left(\frac{n}{12}\right) \\
& +\frac{241847}{350550} \Delta_{3,8}(n)-\frac{58754429}{175275} \Delta_{3,8}\left(\frac{n}{2}\right)-\frac{2314588792}{175275} \Delta_{3,8}\left(\frac{n}{4}\right)-\frac{6078247552}{175275} \Delta_{3,8}\left(\frac{n}{8}\right) \\
& +\frac{146092}{7695} a_{4}(n)+\frac{503776}{1539} a_{4}\left(\frac{n}{2}\right)+\frac{5215744}{405} a_{4}\left(\frac{n}{4}\right)-12 a_{2}(n)-\frac{8748}{19} a_{2}\left(\frac{n}{3}\right)+24 a_{3}(n) \\
& +\frac{4644}{19} a_{3}\left(\frac{n}{3}\right)+\frac{24768}{19} a_{5}(n)-\frac{2539776}{19} a_{5}\left(\frac{n}{2}\right)-120 a_{6}(n)-\frac{88512}{19} a_{6}\left(\frac{n}{2}\right) \\
& -\frac{87616}{19} a_{7}(n)-\frac{70272}{19} a_{8}(n)+\frac{235392}{19} a_{9}(n) \text {, } \\
& N\left(1^{6}, 3^{6}, 6^{4} ; n\right)=\frac{7}{1672800} \sigma_{7}(n)-\frac{7}{1672800} \sigma_{7}\left(\frac{n}{2}\right)-\frac{549}{557600} \sigma_{7}\left(\frac{n}{3}\right)+\frac{7}{418200} \sigma_{7}\left(\frac{n}{4}\right) \\
& +\frac{549}{557600} \sigma_{7}\left(\frac{n}{6}\right)-\frac{224}{52275} \sigma_{7}\left(\frac{n}{8}\right)-\frac{549}{139400} \sigma_{7}\left(\frac{n}{12}\right)+\frac{17568}{17425} \sigma_{7}\left(\frac{n}{24}\right)-\frac{14488856}{654075} a_{1}(n) \\
& -\frac{529960}{1539} a_{1}\left(\frac{n}{2}\right)-\frac{256086}{8075} a_{1}\left(\frac{n}{3}\right)+\frac{424140352}{218025} a_{1}\left(\frac{n}{4}\right)+\frac{7056}{19} a_{1}\left(\frac{n}{6}\right)+\frac{460662336}{8075} a_{1}\left(\frac{n}{12}\right) \\
& +\frac{69647}{175275} \Delta_{3,8}(n)-\frac{24174826}{58425} \Delta_{3,8}\left(\frac{n}{2}\right)-\frac{2152478072}{175275} \Delta_{3,8}\left(\frac{n}{4}\right)-\frac{1995626624}{58425} \Delta_{3,8}\left(\frac{n}{8}\right) \\
& +\frac{167384}{7695} a_{4}(n)+\frac{573664}{1539} a_{4}\left(\frac{n}{2}\right)+\frac{1637888}{135} a_{4}\left(\frac{n}{4}\right)-12 a_{2}(n)-\frac{7020}{19} a_{2}\left(\frac{n}{3}\right)+24 a_{3}(n) \\
& +\frac{864}{19} a_{3}\left(\frac{n}{3}\right)+\frac{26496}{19} a_{5}(n)-\frac{2381184}{19} a_{5}\left(\frac{n}{2}\right)-144 a_{6}(n)-\frac{83008}{19} a_{6}\left(\frac{n}{2}\right) \\
& -\frac{92032}{19} a_{7}(n)-\frac{64768}{19} a_{8}(n)+\frac{241408}{19} a_{9}(n) \text {, } \\
& N\left(1^{6}, 3^{8}, 6^{2} ; n\right)=\frac{7}{836400} \sigma_{7}(n)-\frac{7}{836400} \sigma_{7}\left(\frac{n}{2}\right)-\frac{549}{278800} \sigma_{7}\left(\frac{n}{3}\right)+\frac{7}{418200} \sigma_{7}\left(\frac{n}{4}\right) \\
& +\frac{549}{278800} \sigma_{7}\left(\frac{n}{6}\right)-\frac{224}{52275} \sigma_{7}\left(\frac{n}{8}\right)-\frac{549}{139400} \sigma_{7}\left(\frac{n}{12}\right)+\frac{17568}{17425} \sigma_{7}\left(\frac{n}{24}\right)-\frac{16388362}{654075} a_{1}(n) \\
& -\frac{563096}{1539} a_{1}\left(\frac{n}{2}\right)-\frac{994122}{8075} a_{1}\left(\frac{n}{3}\right)+\frac{424140352}{218025} a_{1}\left(\frac{n}{4}\right)-\frac{6624}{19} a_{1}\left(\frac{n}{6}\right)+\frac{460662336}{8075} a_{1}\left(\frac{n}{12}\right) \\
& +\frac{34744}{175275} \Delta_{3,8}(n)-\frac{33417452}{58425} \Delta_{3,8}\left(\frac{n}{2}\right)-\frac{2232247672}{175275} \Delta_{3,8}\left(\frac{n}{4}\right)-\frac{1995626624}{58425} \Delta_{3,8}\left(\frac{n}{8}\right) \\
& +\frac{191248}{7695} a_{4}(n)+\frac{768224}{1539} a_{4}\left(\frac{n}{2}\right)+\frac{1637888}{135} a_{4}\left(\frac{n}{4}\right)-12 a_{2}(n)-\frac{7020}{19} a_{2}\left(\frac{n}{3}\right)+24 a_{3}(n) \\
& +\frac{864}{19} a_{3}\left(\frac{n}{3}\right)+\frac{6432}{19} a_{5}(n)-\frac{2381184}{19} a_{5}\left(\frac{n}{2}\right)-192 a_{6}(n)-\frac{83008}{19} a_{6}\left(\frac{n}{2}\right) \\
& -\frac{92032}{19} a_{7}(n)-\frac{64768}{19} a_{8}(n)+\frac{241408}{19} a_{9}(n) \text {, }
\end{aligned}
$$




$$
\begin{aligned}
& N\left(1^{6}, 3^{10} ; n\right)=\frac{7}{418200} \sigma_{7}(n)-\frac{549}{139400} \sigma_{7}\left(\frac{n}{3}\right)-\frac{224}{52275} \sigma_{7}\left(\frac{n}{4}\right) \\
& +\frac{17568}{17425} \sigma_{7}\left(\frac{n}{12}\right)-\frac{633136}{34425} a_{1}(n)-\frac{2375296}{34425} a_{1}\left(\frac{n}{2}\right)-\frac{290016}{425} a_{1}\left(\frac{n}{3}\right) \\
& -\frac{706176}{425} a_{1}\left(\frac{n}{4}\right)+\frac{16432}{9225} a_{1}\left(\frac{n}{12}\right)-\frac{31664}{25} \Delta_{3,8}(n)-\frac{20328448}{9225} \Delta_{3,8}\left(\frac{n}{2}\right) \\
& +\frac{11584}{405} \Delta_{3,8}\left(\frac{n}{8}\right)+\frac{385024}{405} a_{4}(n)-8448 a_{3}\left(\frac{n}{3}\right)-384 a_{5}\left(\frac{n}{2}\right), \\
& N\left(1^{6}, 2^{2}, 6^{8} ; n\right)=\frac{1}{669120} \sigma_{7}(n)-\frac{1}{669120} \sigma_{7}\left(\frac{n}{2}\right)+\frac{27}{223040} \sigma_{7}\left(\frac{n}{3}\right) \\
& -\frac{1}{20910} \sigma_{7}\left(\frac{n}{4}\right)-\frac{27}{223040} \sigma_{7}\left(\frac{n}{6}\right)+\frac{128}{10455} \sigma_{7}\left(\frac{n}{8}\right)-\frac{27}{6970} \sigma_{7}\left(\frac{n}{12}\right) \\
& +\frac{3456}{3485} \sigma_{7}\left(\frac{n}{24}\right)-\frac{445357}{29070} a_{1}(n)-\frac{821264}{2565} a_{1}\left(\frac{n}{2}\right)-\frac{397413}{3230} a_{1}\left(\frac{n}{3}\right) \\
& +\frac{17526016}{8721} a_{1}\left(\frac{n}{4}\right)-\frac{106272}{95} a_{1}\left(\frac{n}{6}\right)+\frac{19650816}{323} a_{1}\left(\frac{n}{12}\right)+\frac{17741}{11685} \Delta_{3,8}(n) \\
& -\frac{2651458}{11685} \Delta_{3,8}\left(\frac{n}{2}\right)-\frac{188942704}{11685} \Delta_{3,8}\left(\frac{n}{4}\right)-\frac{84021248}{2337} \Delta_{3,8}\left(\frac{n}{8}\right) \\
& +\frac{2360}{171} a_{4}(n)+\frac{146368}{513} a_{4}\left(\frac{n}{2}\right)+\frac{409600}{27} a_{4}\left(\frac{n}{4}\right)-10 a_{2}(n)-\frac{11016}{19} a_{2}\left(\frac{n}{3}\right) \\
& +22 a_{3}(n)+\frac{11016}{19} a_{3}\left(\frac{n}{3}\right)+\frac{6768}{19} a_{5}(n)-\frac{3027456}{19} a_{5}\left(\frac{n}{2}\right)-88 a_{6}(n) \\
& -\frac{104704}{19} a_{6}\left(\frac{n}{2}\right)-\frac{77184}{19} a_{7}(n)-\frac{90112}{19} a_{8}(n)+\frac{228864}{19} a_{9}(n), \\
& N\left(1^{6}, 2^{2}, 3^{2}, 6^{6} ; n\right)=\frac{1}{334560} \sigma_{7}(n)-\frac{1}{334560} \sigma_{7}\left(\frac{n}{2}\right)+\frac{27}{111520} \sigma_{7}\left(\frac{n}{3}\right) \\
& -\frac{1}{20910} \sigma_{7}\left(\frac{n}{4}\right)-\frac{27}{111520} \sigma_{7}\left(\frac{n}{6}\right)+\frac{128}{10455} \sigma_{7}\left(\frac{n}{8}\right)-\frac{27}{6970} \sigma_{7}\left(\frac{n}{12}\right) \\
& +\frac{3456}{3485} \sigma_{7}\left(\frac{n}{24}\right)-\frac{905791}{87210} a_{1}(n)-\frac{56908}{171} a_{1}\left(\frac{n}{2}\right)-\frac{846693}{3230} a_{1}\left(\frac{n}{3}\right) \\
& +\frac{18374656}{8721} a_{1}\left(\frac{n}{4}\right)-\frac{78516}{19} a_{1}\left(\frac{n}{6}\right)+\frac{14715648}{323} a_{1}\left(\frac{n}{12}\right)+\frac{25199}{7790} \Delta_{3,8}(n) \\
& -\frac{2493719}{11685} \Delta_{3,8}\left(\frac{n}{2}\right)-\frac{16738208}{779} \Delta_{3,8}\left(\frac{n}{4}\right)-\frac{89720576}{2337} \Delta_{3,8}\left(\frac{n}{8}\right) \\
& +\frac{3668}{513} a_{4}(n)+\frac{63616}{171} a_{4}\left(\frac{n}{2}\right)+\frac{504832}{27} a_{4}\left(\frac{n}{4}\right)-8 a_{2}(n)-\frac{14256}{19} a_{2}\left(\frac{n}{3}\right) \\
& +20 a_{3}(n)+\frac{20412}{19} a_{3}\left(\frac{n}{3}\right)-\frac{41616}{19} a_{5}(n)-\frac{3732480}{19} a_{5}\left(\frac{n}{2}\right)-80 a_{6}(n) \\
& -\frac{132352}{19} a_{6}\left(\frac{n}{2}\right)-\frac{62976}{19} a_{7}(n)-\frac{122624}{19} a_{8}(n)+\frac{225792}{19} a_{9}(n),
\end{aligned}
$$




$$
\begin{aligned}
& N\left(1^{6}, 2^{2}, 3^{4}, 6^{4} ; n\right)=\frac{1}{167280} \sigma_{7}(n)-\frac{1}{167280} \sigma_{7}\left(\frac{n}{2}\right)+\frac{27}{55760} \sigma_{7}\left(\frac{n}{3}\right)-\frac{1}{20910} \sigma_{7}\left(\frac{n}{4}\right) \\
& -\frac{27}{55760} \sigma_{7}\left(\frac{n}{6}\right)+\frac{128}{10455} \sigma_{7}\left(\frac{n}{8}\right)-\frac{27}{6970} \sigma_{7}\left(\frac{n}{12}\right)+\frac{3456}{3485} \sigma_{7}\left(\frac{n}{24}\right)-\frac{191638}{43605} a_{1}(n) \\
& -\frac{62896}{171} a_{1}\left(\frac{n}{2}\right)-\frac{680994}{1615} a_{1}\left(\frac{n}{3}\right)+\frac{20537600}{8721} a_{1}\left(\frac{n}{4}\right)-\frac{136512}{19} a_{1}\left(\frac{n}{6}\right)+\frac{9075456}{323} a_{1}\left(\frac{n}{12}\right) \\
& +\frac{60796}{11685} \Delta_{3,8}(n)-\frac{1944664}{11685} \Delta_{3,8}\left(\frac{n}{2}\right)-\frac{23152576}{779} \Delta_{3,8}\left(\frac{n}{4}\right)-\frac{102473216}{2337} \Delta_{3,8}\left(\frac{n}{8}\right) \\
& -\frac{416}{513} a_{4}(n)+\frac{78592}{171} a_{4}\left(\frac{n}{2}\right)+\frac{665600}{27} a_{4}\left(\frac{n}{4}\right)-4 a_{2}(n)-\frac{16200}{19} a_{2}\left(\frac{n}{3}\right)+16 a_{3}(n) \\
& +\frac{28512}{19} a_{3}\left(\frac{n}{3}\right)-\frac{99648}{19} a_{5}(n)-\frac{4925952}{19} a_{5}\left(\frac{n}{2}\right)-64 a_{6}(n)-\frac{177152}{19} a_{6}\left(\frac{n}{2}\right) \\
& -\frac{52992}{19} a_{7}(n)-\frac{177152}{19} a_{8}(n)+\frac{247296}{19} a_{9}(n) \text {, } \\
& N\left(1^{6}, 2^{2}, 3^{2}, 6^{6} ; n\right)=\frac{1}{83640} \sigma_{7}(n)-\frac{1}{83640} \sigma_{7}\left(\frac{n}{2}\right)+\frac{27}{27880} \sigma_{7}\left(\frac{n}{3}\right)-\frac{1}{20910} \sigma_{7}\left(\frac{n}{4}\right) \\
& -\frac{27}{27880} \sigma_{7}\left(\frac{n}{6}\right)+\frac{128}{10455} \sigma_{7}\left(\frac{n}{8}\right)-\frac{27}{6970} \sigma_{7}\left(\frac{n}{12}\right)+\frac{3456}{3485} \sigma_{7}\left(\frac{n}{24}\right)+\frac{174766}{43605} a_{1}(n) \\
& -\frac{1129384}{2565} a_{1}\left(\frac{n}{2}\right)-\frac{997542}{1615} a_{1}\left(\frac{n}{3}\right)+\frac{40702208}{14535} a_{1}\left(\frac{n}{4}\right)-\frac{1053432}{95} a_{1}\left(\frac{n}{6}\right)-\frac{15959808}{1615} a_{1}\left(\frac{n}{12}\right) \\
& +\frac{89038}{11685} \Delta_{3,8}(n)-\frac{236292}{3895} \Delta_{3,8}\left(\frac{n}{2}\right)-\frac{501220352}{11685} \Delta_{3,8}\left(\frac{n}{4}\right)-\frac{624652288}{11685} \Delta_{3,8}\left(\frac{n}{8}\right) \\
& -\frac{5968}{513} a_{4}(n)+\frac{284672}{513} a_{4}\left(\frac{n}{2}\right)+\frac{311296}{9} a_{4}\left(\frac{n}{4}\right)+4 a_{2}(n)-\frac{14580}{19} a_{2}\left(\frac{n}{3}\right)+8 a_{3}(n) \\
& +\frac{33048}{19} a_{3}\left(\frac{n}{3}\right)-\frac{173952}{19} a_{5}(n)-\frac{6893568}{19} a_{5}\left(\frac{n}{2}\right)-32 a_{6}(n)-\frac{250880}{19} a_{6}\left(\frac{n}{2}\right) \\
& -\frac{49152}{19} a_{7}(n)-\frac{270336}{19} a_{8}(n)+\frac{307200}{19} a_{9}(n) \text {, } \\
& N\left(1^{6}, 2^{2}, 3^{8} ; n\right)=\frac{1}{41820} \sigma_{7}(n)-\frac{1}{41820} \sigma_{7}\left(\frac{n}{2}\right)+\frac{27}{13940} \sigma_{7}\left(\frac{n}{3}\right)-\frac{1}{20910} \sigma_{7}\left(\frac{n}{4}\right) \\
& -\frac{27}{13940} \sigma_{7}\left(\frac{n}{6}\right)+\frac{128}{10455} \sigma_{7}\left(\frac{n}{8}\right)-\frac{27}{6970} \sigma_{7}\left(\frac{n}{12}\right)+\frac{3456}{3485} \sigma_{7}\left(\frac{n}{24}\right)+\frac{910124}{43605} a_{1}(n) \\
& -\frac{429904}{855} a_{1}\left(\frac{n}{2}\right)-\frac{1497528}{1615} a_{1}\left(\frac{n}{3}\right)+\frac{26804480}{8721} a_{1}\left(\frac{n}{4}\right)-\frac{1584576}{95} a_{1}\left(\frac{n}{6}\right)-\frac{36986112}{323} a_{1}\left(\frac{n}{12}\right) \\
& +\frac{44544}{3895} \Delta_{3,8}(n)+\frac{73904}{2337} \Delta_{3,8}\left(\frac{n}{2}\right)-\frac{737977312}{11685} \Delta_{3,8}\left(\frac{n}{4}\right)-\frac{143701504}{2337} \Delta_{3,8}\left(\frac{n}{8}\right) \\
& -\frac{14528}{513} a_{4}(n)+\frac{129152}{171} a_{4}\left(\frac{n}{2}\right)+\frac{1329152}{27} a_{4}\left(\frac{n}{4}\right)+24 a_{2}(n)-\frac{1296}{19} a_{2}\left(\frac{n}{3}\right)-16 a_{3}(n) \\
& +\frac{25920}{19} a_{3}\left(\frac{n}{3}\right)-\frac{321408}{19} a_{5}(n)+-\frac{9755136}{19} a_{5}\left(\frac{n}{2}\right)-\frac{362240}{19} a_{6}\left(\frac{n}{2}\right)-\frac{32256}{19} a_{7}(n) \\
& -\frac{435200}{19} a_{8}(n)+\frac{384000}{19} a_{9}(n) \text {, }
\end{aligned}
$$




$$
\begin{aligned}
& N\left(1^{6}, 2^{4}, 6^{6} ; n\right)=\frac{61}{13382400} \sigma_{7}(n)-\frac{61}{13382400} \sigma_{7}\left(\frac{n}{2}\right)-\frac{567}{4460800} \sigma_{7}\left(\frac{n}{3}\right)+\frac{61}{418200} \sigma_{7}\left(\frac{n}{4}\right) \\
& +\frac{567}{4460800} \sigma_{7}\left(\frac{n}{6}\right)-\frac{1952}{52275} \sigma_{7}\left(\frac{n}{8}\right)-\frac{567}{139400} \sigma_{7}\left(\frac{n}{12}\right)+\frac{18144}{17425} \sigma_{7}\left(\frac{n}{24}\right)-\frac{100712273}{5232600} a_{1}(n) \\
& -\frac{128368}{513} a_{1}\left(\frac{n}{2}\right)-\frac{3284163}{64600} a_{1}\left(\frac{n}{3}\right)+\frac{1014607168}{654075} a_{1}\left(\frac{n}{4}\right)-\frac{13464}{19} a_{1}\left(\frac{n}{6}\right)+\frac{638371008}{8075} a_{1}\left(\frac{n}{12}\right) \\
& +\frac{440563}{701100} \Delta_{3,8}(n)-\frac{117679931}{350550} \Delta_{3,8}\left(\frac{n}{2}\right)-\frac{1157563516}{175275} \Delta_{3,8}\left(\frac{n}{4}\right)-\frac{4665372416}{175275} \Delta_{3,8}\left(\frac{n}{8}\right) \\
& +\frac{143254}{7695} a_{4}(n)+\frac{142480}{513} a_{4}\left(\frac{n}{2}\right)+\frac{2941952}{405} a_{4}\left(\frac{n}{4}\right)-16 a_{2}(n)-\frac{16254}{19} a_{2}\left(\frac{n}{3}\right)+28 a_{3}(n) \\
& +\frac{16254}{19} a_{3}\left(\frac{n}{3}\right)+\frac{39492}{19} a_{5}(n)-\frac{1454592}{19} a_{5}\left(\frac{n}{2}\right)-114 a_{6}(n)-\frac{48960}{19} a_{6}\left(\frac{n}{2}\right)-\frac{62240}{19} a_{7}(n) \\
& -\frac{18560}{19} a_{8}(n)+\frac{151424}{19} a_{9}(n) \text {, } \\
& N\left(1^{6}, 2^{4}, 3^{2}, 6^{4} ; n\right)=\frac{61}{6691200} \sigma_{7}(n)-\frac{61}{6691200} \sigma_{7}\left(\frac{n}{2}\right)-\frac{567}{2230400} \sigma_{7}\left(\frac{n}{3}\right)+\frac{61}{418200} \sigma_{7}\left(\frac{n}{4}\right) \\
& +\frac{567}{2230400} \sigma_{7}\left(\frac{n}{6}\right)-\frac{1952}{52275} \sigma_{7}\left(\frac{n}{8}\right)-\frac{567}{139400} \sigma_{7}\left(\frac{n}{12}\right)+\frac{18144}{17425} \sigma_{7}\left(\frac{n}{24}\right)+\frac{87035603}{2616300} a_{1}(n) \\
& -\frac{218852}{855} a_{1}\left(\frac{n}{2}\right)+\frac{11188107}{32300} a_{1}\left(\frac{n}{3}\right)+\frac{934203968}{654075} a_{1}\left(\frac{n}{4}\right)+\frac{656892}{95} a_{1}\left(\frac{n}{6}\right)+\frac{774479808}{8075} a_{1}\left(\frac{n}{12}\right) \\
& -\frac{749266}{175275} \Delta_{3,8}(n)-\frac{61804106}{175275} \Delta_{3,8}\left(\frac{n}{2}\right)+\frac{973651744}{175275} \Delta_{3,8}\left(\frac{n}{4}\right)-\frac{3992972416}{175275} \Delta_{3,8}\left(\frac{n}{8}\right) \\
& +\frac{288848}{7695} a_{4}(n)+\frac{7552}{171} a_{4}\left(\frac{n}{2}\right)-\frac{86528}{405} a_{4}\left(\frac{n}{4}\right)-20 a_{2}(n)-\frac{3348}{19} a_{2}\left(\frac{n}{3}\right)+32 a_{3}(n) \\
& -\frac{15120}{19} a_{3}\left(\frac{n}{3}\right)+\frac{177912}{19} a_{5}(n)+\frac{87168}{19} a_{5}\left(\frac{n}{2}\right)-132 a_{6}(n)+\frac{11072}{19} a_{6}\left(\frac{n}{2}\right) \\
& -\frac{110080}{19} a_{7}(n)+\frac{51200}{19} a_{8}(n)+\frac{199168}{19} a_{9}(n) \text {, } \\
& N\left(1^{6}, 2^{4}, 3^{4}, 6^{2} ; n\right)=\frac{61}{3345600} \sigma_{7}(n)-\frac{61}{3345600} \sigma_{7}\left(\frac{n}{2}\right)-\frac{567}{1115200} \sigma_{7}\left(\frac{n}{3}\right)+\frac{61}{418200} \sigma_{7}\left(\frac{n}{4}\right) \\
& +\frac{567}{1115200} \sigma_{7}\left(\frac{n}{6}\right)-\frac{1952}{52275} \sigma_{7}\left(\frac{n}{8}\right)-\frac{567}{139400} \sigma_{7}\left(\frac{n}{12}\right)+\frac{18144}{17425} \sigma_{7}\left(\frac{n}{24}\right)-\frac{31990484}{654075} a_{1}(n) \\
& -\frac{490172}{2565} a_{1}\left(\frac{n}{2}\right)+\frac{6358671}{8075} a_{1}\left(\frac{n}{3}\right)+\frac{568701248}{654075} a_{1}\left(\frac{n}{4}\right)+\frac{1406844}{95} a_{1}\left(\frac{n}{6}\right)+\frac{1025057088}{8075} a_{1}\left(\frac{n}{12}\right) \\
& -\frac{3406859}{350550} \Delta_{3,8}(n)-\frac{80141447}{175275} \Delta_{3,8}\left(\frac{n}{2}\right)+\frac{4453297144}{175275} \Delta_{3,8}\left(\frac{n}{4}\right)-\frac{1805973376}{175275} \Delta_{3,8}\left(\frac{n}{8}\right) \\
& +\frac{451076}{7695} a_{4}(n)-\frac{96416}{513} a_{4}\left(\frac{n}{2}\right)-\frac{5815808}{405} a_{4}\left(\frac{n}{4}\right)-28 a_{2}(n)+\frac{5724}{19} a_{2}\left(\frac{n}{3}\right)+40 a_{3}(n) \\
& -\frac{42660}{19} a_{3}\left(\frac{n}{3}\right)+\frac{330624}{19} a_{5}(n)+\frac{2949888}{19} a_{5}\left(\frac{n}{2}\right)-168 a_{6}(n)+\frac{117184}{19} a_{6}\left(\frac{n}{2}\right) \\
& -\frac{143296}{19} a_{7}(n)+\frac{176768}{19} a_{8}(n)+\frac{174208}{19} a_{9}(n) \text {, }
\end{aligned}
$$




$$
\begin{aligned}
& N\left(1^{6}, 2^{4}, 3^{6} ; n\right)=\frac{61}{1672800} \sigma_{7}(n)-\frac{61}{1672800} \sigma_{7}\left(\frac{n}{2}\right)-\frac{567}{557600} \sigma_{7}\left(\frac{n}{3}\right)+\frac{61}{418200} \sigma_{7}\left(\frac{n}{4}\right) \\
& +\frac{567}{557600} \sigma_{7}\left(\frac{n}{6}\right)-\frac{1952}{52275} \sigma_{7}\left(\frac{n}{8}\right)-\frac{567}{139400} \sigma_{7}\left(\frac{n}{12}\right)+\frac{18144}{17425} \sigma_{7}\left(\frac{n}{24}\right)-\frac{15350356}{218025} a_{1}(n) \\
& -\frac{63128}{513} a_{1}\left(\frac{n}{2}\right)+\frac{10315242}{8075} a_{1}\left(\frac{n}{3}\right)+\frac{17118272}{72675} a_{1}\left(\frac{n}{4}\right)+\frac{429408}{19} a_{1}\left(\frac{n}{6}\right)+\frac{1541585088}{8075} a_{1}\left(\frac{n}{12}\right) \\
& -\frac{921053}{58425} \Delta_{3,8}(n)-\frac{40345078}{58425} \Delta_{3,8}\left(\frac{n}{2}\right)+\frac{2957443208}{58425} \Delta_{3,8}\left(\frac{n}{4}\right)+\frac{259643008}{58425} \Delta_{3,8}\left(\frac{n}{8}\right) \\
& +\frac{220984}{2565} a_{4}(n)-\frac{190240}{513} a_{4}\left(\frac{n}{2}\right)-\frac{1475072}{45} a_{4}\left(\frac{n}{4}\right)-44 a_{2}(n)+\frac{8100}{19} a_{2}\left(\frac{n}{3}\right)+56 a_{3}(n) \\
& -\frac{63504}{19} a_{3}\left(\frac{n}{3}\right)+\frac{514944}{19} a_{5}(n)+\frac{6643584}{19} a_{5}\left(\frac{n}{2}\right)-240 a_{6}(n)+\frac{252736}{19} a_{6}\left(\frac{n}{2}\right) \\
& -\frac{177792}{19} a_{7}(n)+\frac{346368}{19} a_{8}(n)+\frac{109824}{19} a_{9}(n) \text {, } \\
& N\left(1^{6}, 2^{6}, 6^{4} ; n\right)=\frac{91}{6691200} \sigma_{7}(n)-\frac{91}{6691200} \sigma_{7}\left(\frac{n}{2}\right)+\frac{243}{2230400} \sigma_{7}\left(\frac{n}{3}\right)-\frac{91}{209100} \sigma_{7}\left(\frac{n}{4}\right) \\
& -\frac{243}{2230400} \sigma_{7}\left(\frac{n}{6}\right)+\frac{5824}{52275} \sigma_{7}\left(\frac{n}{8}\right)-\frac{243}{69700} \sigma_{7}\left(\frac{n}{12}\right)+\frac{15552}{17425} \sigma_{7}\left(\frac{n}{24}\right)-\frac{35801123}{2616300} a_{1}(n) \\
& -\frac{934928}{2565} a_{1}\left(\frac{n}{2}\right)-\frac{3979413}{32300} a_{1}\left(\frac{n}{3}\right)+\frac{1452566144}{654075} a_{1}\left(\frac{n}{4}\right)-\frac{105264}{95} a_{1}\left(\frac{n}{6}\right)+\frac{300276864}{8075} a_{1}\left(\frac{n}{12}\right) \\
& +\frac{532963}{350550} \Delta_{3,8}(n)-\frac{17572151}{175275} \Delta_{3,8}\left(\frac{n}{2}\right)-\frac{3722161448}{175275} \Delta_{3,8}\left(\frac{n}{4}\right)-\frac{7108630528}{175275} \Delta_{3,8}\left(\frac{n}{8}\right) \\
& +\frac{93548}{7695} a_{4}(n)+\frac{114784}{513} a_{4}\left(\frac{n}{2}\right)+\frac{8052736}{405} a_{4}\left(\frac{n}{4}\right)-6 a_{2}(n)-\frac{5940}{19} a_{2}\left(\frac{n}{3}\right)+18 a_{3}(n) \\
& +\frac{5940}{19} a_{3}\left(\frac{n}{3}\right)+\frac{11160}{19} a_{5}(n)-\frac{3948288}{19} a_{5}\left(\frac{n}{2}\right)-60 a_{6}(n)-\frac{135808}{19} a_{6}\left(\frac{n}{2}\right)-\frac{83392}{19} a_{7}(n) \\
& -\frac{128512}{19} a_{8}(n)+\frac{281344}{19} a_{9}(n) \text {, } \\
& N\left(1^{6}, 2^{6}, 3^{2}, 6^{2} ; n\right)=\frac{91}{3345600} \sigma_{7}(n)-\frac{91}{3345600} \sigma_{7}\left(\frac{n}{2}\right)+\frac{243}{1115200} \sigma_{7}\left(\frac{n}{3}\right)-\frac{91}{209100} \sigma_{7}\left(\frac{n}{4}\right) \\
& -\frac{243}{1115200} \sigma_{7}\left(\frac{n}{6}\right)+\frac{5824}{52275} \sigma_{7}\left(\frac{n}{8}\right)-\frac{243}{69700} \sigma_{7}\left(\frac{n}{12}\right)+\frac{15552}{17425} \sigma_{7}\left(\frac{n}{24}\right)+\frac{16506461}{654075} a_{1}(n) \\
& -\frac{726196}{2565} a_{1}\left(\frac{n}{2}\right)-\frac{10193184}{8075} a_{1}\left(\frac{n}{3}\right)+\frac{1540672384}{654075} a_{1}\left(\frac{n}{4}\right)-\frac{2039508}{95} a_{1}\left(\frac{n}{6}\right)+\frac{282847104}{8075} a_{1}\left(\frac{n}{12}\right) \\
& +\frac{5462561}{350550} \Delta_{3,8}(n)-\frac{19168447}{175275} \Delta_{3,8}\left(\frac{n}{2}\right)-\frac{8903306848}{175275} \Delta_{3,8}\left(\frac{n}{4}\right)-\frac{8232017408}{175275} \Delta_{3,8}\left(\frac{n}{8}\right) \\
& -\frac{314204}{7695} a_{4}(n)+\frac{453248}{513} a_{4}\left(\frac{n}{2}\right)+\frac{14944256}{405} a_{4}\left(\frac{n}{4}\right)-\frac{50760}{19} a_{2}\left(\frac{n}{3}\right)+12 a_{3}(n) \\
& +\frac{106164}{19} a_{3}\left(\frac{n}{3}\right)-\frac{385920}{19} a_{5}(n)-\frac{7557888}{19} a_{5}\left(\frac{n}{2}\right)-24 a_{6}(n)-\frac{275072}{19} a_{6}\left(\frac{n}{2}\right) \\
& +\frac{69376}{19} a_{7}(n)-\frac{282368}{19} a_{8}(n)+\frac{88064}{19} a_{9}(n) \text {, }
\end{aligned}
$$




$$
\begin{aligned}
& N\left(1^{6}, 2^{6}, 3^{4} ; n\right)=\frac{91}{1672800} \sigma_{7}(n)-\frac{91}{1672800} \sigma_{7}\left(\frac{n}{2}\right)+\frac{243}{557600} \sigma_{7}\left(\frac{n}{3}\right)-\frac{91}{209100} \sigma_{7}\left(\frac{n}{4}\right) \\
& -\frac{243}{557600} \sigma_{7}\left(\frac{n}{6}\right)+\frac{5824}{52275} \sigma_{7}\left(\frac{n}{8}\right)-\frac{243}{69700} \sigma_{7}\left(\frac{n}{12}\right)+\frac{15552}{17425} \sigma_{7}\left(\frac{n}{24}\right)+\frac{42606362}{654075} a_{1}(n) \\
& -\frac{183224}{513} a_{1}\left(\frac{n}{2}\right)-\frac{20190528}{8075} a_{1}\left(\frac{n}{3}\right)+\frac{2273701504}{654075} a_{1}\left(\frac{n}{4}\right)-\frac{785880}{19} a_{1}\left(\frac{n}{6}\right)+\frac{48426624}{8075} a_{1}\left(\frac{n}{12}\right) \\
& +\frac{5410081}{175275} \Delta_{3,8}(n)+\frac{15791306}{175275} \Delta_{3,8}\left(\frac{n}{2}\right)-\frac{16792084048}{175275} \Delta_{3,8}\left(\frac{n}{4}\right)-\frac{12745979648}{175275} \Delta_{3,8}\left(\frac{n}{8}\right) \\
& -\frac{738968}{7695} a_{4}(n)+\frac{763328}{513} a_{4}\left(\frac{n}{2}\right)+\frac{27616256}{405} a_{4}\left(\frac{n}{4}\right)+12 a_{2}(n)-\frac{87696}{19} a_{2}\left(\frac{n}{3}\right) \\
& +\frac{198504}{19} a_{3}\left(\frac{n}{3}\right)-\frac{779904}{19} a_{5}(n)-\frac{13992960}{19} a_{5}\left(\frac{n}{2}\right)+48 a_{6}(n)-\frac{508544}{19} a_{6}\left(\frac{n}{2}\right) \\
& +\frac{178816}{19} a_{7}(n)-\frac{545024}{19} a_{8}(n)+\frac{44288}{19} a_{9}(n) \text {, } \\
& N\left(1^{6}, 2^{8}, 6^{2} ; n\right)=\frac{547}{13382400} \sigma_{7}(n)-\frac{547}{13382400} \sigma_{7}\left(\frac{n}{2}\right)-\frac{729}{4460800} \sigma_{7}\left(\frac{n}{3}\right)+\frac{547}{418200} \sigma_{7}\left(\frac{n}{4}\right) \\
& +\frac{729}{4460800} \sigma_{7}\left(\frac{n}{6}\right)-\frac{17504}{52275} \sigma_{7}\left(\frac{n}{8}\right)-\frac{729}{139400} \sigma_{7}\left(\frac{n}{12}\right)+\frac{23328}{17425} \sigma_{7}\left(\frac{n}{24}\right)-\frac{14234719}{581400} a_{1}(n) \\
& -\frac{674032}{2565} a_{1}\left(\frac{n}{2}\right)-\frac{1675701}{64600} a_{1}\left(\frac{n}{3}\right)+\frac{358471232}{218025} a_{1}\left(\frac{n}{4}\right)-\frac{131976}{95} a_{1}\left(\frac{n}{6}\right)+\frac{883123776}{8075} a_{1}\left(\frac{n}{12}\right) \\
& +\frac{75067}{233700} \Delta_{3,8}(n)-\frac{59378699}{116850} \Delta_{3,8}\left(\frac{n}{2}\right)-\frac{197732044}{58425} \Delta_{3,8}\left(\frac{n}{4}\right)-\frac{1591510784}{58425} \Delta_{3,8}\left(\frac{n}{8}\right) \\
& +\frac{20642}{855} a_{4}(n)+\frac{199664}{513} a_{4}\left(\frac{n}{2}\right)+\frac{590848}{135} a_{4}\left(\frac{n}{4}\right)-20 a_{2}(n)-\frac{23490}{19} a_{2}\left(\frac{n}{3}\right) \\
& +32 a_{3}(n)+\frac{23490}{19} a_{3}\left(\frac{n}{3}\right)+\frac{41148}{19} a_{5}(n)-\frac{887040}{19} a_{5}\left(\frac{n}{2}\right)-158 a_{6}(n)-\frac{31424}{19} a_{6}\left(\frac{n}{2}\right) \\
& -\frac{61152}{19} a_{7}(n)+\frac{11136}{19} a_{8}(n)+\frac{145536}{19} a_{9}(n), \\
& N\left(1^{6}, 2^{8}, 3^{2} ; n\right)=\frac{547}{6691200} \sigma_{7}(n)+-\frac{547}{6691200} \sigma_{7}\left(\frac{n}{2}\right)-\frac{729}{2230400} \sigma_{7}\left(\frac{n}{3}\right)+\frac{547}{418200} \sigma_{7}\left(\frac{n}{4}\right) \\
& +\frac{729}{2230400} \sigma_{7}\left(\frac{n}{6}\right)-\frac{17504}{52275} \sigma_{7}\left(\frac{n}{8}\right)-\frac{729}{139400} \sigma_{7}\left(\frac{n}{12}\right)+\frac{23328}{17425} \sigma_{7}\left(\frac{n}{24}\right)-\frac{114444667}{872100} a_{1}(n) \\
& -\frac{1137964}{2565} a_{1}\left(\frac{n}{2}\right)+\frac{107442369}{32300} a_{1}\left(\frac{n}{3}\right)+\frac{60528064}{72675} a_{1}\left(\frac{n}{4}\right)+\frac{5555628}{95} a_{1}\left(\frac{n}{6}\right)+\frac{497710656}{8075} a_{1}\left(\frac{n}{12}\right) \\
& -\frac{799733}{19475} \Delta_{3,8}(n)-\frac{7041148}{19475} \Delta_{3,8}\left(\frac{n}{2}\right)+\frac{1624609792}{19475} \Delta_{3,8}\left(\frac{n}{4}\right)-\frac{31095168}{19475} \Delta_{3,8}\left(\frac{n}{8}\right) \\
& +\frac{441832}{2565} a_{4}(n)-\frac{838144}{513} a_{4}\left(\frac{n}{2}\right)-\frac{2082304}{45} a_{4}\left(\frac{n}{4}\right)-28 a_{2}(n)+\frac{119556}{19} a_{2}\left(\frac{n}{3}\right)+40 a_{3}(n) \\
& -\frac{285768}{19} a_{3}\left(\frac{n}{3}\right)+\frac{1188648}{19} a_{5}(n)+\frac{9901440}{19} a_{5}\left(\frac{n}{2}\right)-220 a_{6}(n)+\frac{380352}{19} a_{6}\left(\frac{n}{2}\right) \\
& -\frac{504576}{19} a_{7}(n)+\frac{442368}{19} a_{8}(n)+\frac{691200}{19} a_{9}(n) \text {, }
\end{aligned}
$$




$$
\begin{aligned}
& N\left(1^{6}, 2^{10} ; n\right)=\frac{1}{8160} \sigma_{7}(n)-\frac{1}{8160} \sigma_{7}\left(\frac{n}{2}\right)-\frac{1}{255} \sigma_{7}\left(\frac{n}{4}\right)+\frac{256}{255} \sigma_{7}\left(\frac{n}{8}\right) \\
& -\frac{1}{17} a_{1}(n)-16 a_{1}\left(\frac{n}{2}\right)-\frac{512}{17} a_{1}\left(\frac{n}{4}\right)-2 a_{2}(n)+14 a_{3}(n), \\
& N\left(1^{7}, 2,3,6^{7} ; n\right)=\frac{1}{326400} \sigma_{7}(n)-\frac{1}{326400} \sigma_{7}\left(\frac{n}{2}\right)-\frac{27}{108800} \sigma_{7}\left(\frac{n}{3}\right) \\
& +\frac{1}{20400} \sigma_{7}\left(\frac{n}{4}\right)+\frac{27}{108800} \sigma_{7}\left(\frac{n}{6}\right)-\frac{16}{1275} \sigma_{7}\left(\frac{n}{8}\right)-\frac{27}{6800} \sigma_{7}\left(\frac{n}{12}\right) \\
& +\frac{432}{425} \sigma_{7}\left(\frac{n}{24}\right)-\frac{121702433}{5232600} a_{1}(n)-\frac{290078}{855} a_{1}\left(\frac{n}{2}\right)-\frac{1385523}{64600} a_{1}\left(\frac{n}{3}\right) \\
& +\frac{1357834144}{654075} a_{1}\left(\frac{n}{4}\right)+\frac{2178}{95} a_{1}\left(\frac{n}{6}\right)+\frac{677967264}{8075} a_{1}\left(\frac{n}{12}\right) \\
& +\frac{4553}{17100} \Delta_{3,8}(n)-\frac{2936581}{8550} \Delta_{3,8}\left(\frac{n}{2}\right)-\frac{48002168}{4275} \Delta_{3,8}\left(\frac{n}{4}\right) \\
& -\frac{154623808}{4275} \Delta_{3,8}\left(\frac{n}{8}\right)+\frac{176914}{7695} a_{4}(n)+\frac{51808}{171} a_{4}\left(\frac{n}{2}\right) \\
& +\frac{4795136}{405} a_{4}\left(\frac{n}{4}\right)-16 a_{2}(n)-\frac{13986}{19} a_{2}\left(\frac{n}{3}\right)+30 a_{3}(n) \\
& +\frac{10908}{19} a_{3}\left(\frac{n}{3}\right)+\frac{53388}{19} a_{5}(n)-\frac{2353152}{19} a_{5}\left(\frac{n}{2}\right)-126 a_{6}(n) \\
& -\frac{79584}{19} a_{6}\left(\frac{n}{2}\right)-\frac{91520}{19} a_{7}(n)-\frac{48576}{19} a_{8}(n)+\frac{230912}{19} a_{9}(n), \\
& N\left(1^{7}, 2,3^{3}, 6^{5} ; n\right)=\frac{1}{163200} \sigma_{7}(n)-\frac{1}{163200} \sigma_{7}\left(\frac{n}{2}\right)-\frac{27}{54400} \sigma_{7}\left(\frac{n}{3}\right) \\
& +\frac{1}{20400} \sigma_{7}\left(\frac{n}{4}\right)+\frac{27}{54400} \sigma_{7}\left(\frac{n}{6}\right)-\frac{16}{1275} \sigma_{7}\left(\frac{n}{8}\right)-\frac{27}{6800} \sigma_{7}\left(\frac{n}{12}\right) \\
& +\frac{432}{425} \sigma_{7}\left(\frac{n}{24}\right)-\frac{18686492}{654075} a_{1}(n)-\frac{174182}{513} a_{1}\left(\frac{n}{2}\right)+\frac{1839771}{16150} a_{1}\left(\frac{n}{3}\right) \\
& +\frac{1308667424}{654075} a_{1}\left(\frac{n}{4}\right)+\frac{46998}{19} a_{1}\left(\frac{n}{6}\right)+\frac{725360544}{8075} a_{1}\left(\frac{n}{12}\right) \\
& -\frac{23999}{17100} \Delta_{3,8}(n)-\frac{3232787}{8550} \Delta_{3,8}\left(\frac{n}{2}\right)-\frac{27577468}{4275} \Delta_{3,8}\left(\frac{n}{4}\right) \\
& -\frac{146111168}{4275} \Delta_{3,8}\left(\frac{n}{8}\right)+\frac{230618}{7695} a_{4}(n)+\frac{124880}{513} a_{4}\left(\frac{n}{2}\right) \\
& +\frac{3517696}{405} a_{4}\left(\frac{n}{4}\right)-18 a_{2}(n)-\frac{10152}{19} a_{2}\left(\frac{n}{3}\right)+32 a_{3}(n) \\
& +\frac{918}{19} a_{3}\left(\frac{n}{3}\right)+\frac{100656}{19} a_{5}(n)-\frac{1704192}{19} a_{5}\left(\frac{n}{2}\right)-140 a_{6}(n) \\
& -\frac{55136}{19} a_{6}\left(\frac{n}{2}\right)-\frac{106144}{19} a_{7}(n)-\frac{19264}{19} a_{8}(n)+\frac{241600}{19} a_{9}(n),
\end{aligned}
$$




$$
\begin{aligned}
& N\left(1^{7}, 2,3^{5}, 6^{3} ; n\right)=\frac{1}{81600} \sigma_{7}(n)-\frac{1}{81600} \sigma_{7}\left(\frac{n}{2}\right)-\frac{27}{27200} \sigma_{7}\left(\frac{n}{3}\right)+\frac{1}{20400} \sigma_{7}\left(\frac{n}{4}\right) \\
& +\frac{27}{27200} \sigma_{7}\left(\frac{n}{6}\right)-\frac{16}{1275} \sigma_{7}\left(\frac{n}{8}\right)-\frac{27}{6800} \sigma_{7}\left(\frac{n}{12}\right)+\frac{432}{425} \sigma_{7}\left(\frac{n}{24}\right)-\frac{22949504}{654075} a_{1}(n) \\
& -\frac{795472}{2565} a_{1}\left(\frac{n}{2}\right)+\frac{2139651}{8075} a_{1}\left(\frac{n}{3}\right)+\frac{1142497184}{654075} a_{1}\left(\frac{n}{4}\right)+\frac{512604}{95} a_{1}\left(\frac{n}{6}\right) \\
& +\frac{862987104}{8075} a_{1}\left(\frac{n}{12}\right)-\frac{27919}{8550} \Delta_{3,8}(n)-\frac{2002177}{4275} \Delta_{3,8}\left(\frac{n}{2}\right)+\frac{7391732}{4275} \Delta_{3,8}\left(\frac{n}{4}\right) \\
& -\frac{122228288}{4275} \Delta_{3,8}\left(\frac{n}{8}\right)+\frac{295076}{7695} a_{4}(n)+\frac{100112}{513} a_{4}\left(\frac{n}{2}\right)+\frac{1052416}{405} a_{4}\left(\frac{n}{4}\right) \\
& -22 a_{2}(n)-\frac{8370}{19} a_{2}\left(\frac{n}{3}\right)+36 a_{3}(n)-\frac{7020}{19} a_{3}\left(\frac{n}{3}\right)+\frac{151344}{19} a_{5}(n)-\frac{482496}{19} a_{5}\left(\frac{n}{2}\right) \\
& -168 a_{6}(n)-\frac{10016}{19} a_{6}\left(\frac{n}{2}\right)-\frac{114688}{19} a_{7}(n)+\frac{35584}{19} a_{8}(n)+\frac{220672}{19} a_{9}(n), \\
& N\left(1^{7}, 2,3^{7}, 6 ; n\right)=\frac{1}{40800} \sigma_{7}(n)-\frac{1}{40800} \sigma_{7}\left(\frac{n}{2}\right)-\frac{27}{13600} \sigma_{7}\left(\frac{n}{3}\right)+\frac{1}{20400} \sigma_{7}\left(\frac{n}{4}\right) \\
& +\frac{27}{13600} \sigma_{7}\left(\frac{n}{6}\right)-\frac{16}{1275} \sigma_{7}\left(\frac{n}{8}\right)-\frac{27}{6800} \sigma_{7}\left(\frac{n}{12}\right)+\frac{432}{425} \sigma_{7}\left(\frac{n}{24}\right)-\frac{3264617}{72675} a_{1}(n) \\
& -\frac{681412}{2565} a_{1}\left(\frac{n}{2}\right)+\frac{3504357}{8075} a_{1}\left(\frac{n}{3}\right)+\frac{301154528}{218025} a_{1}\left(\frac{n}{4}\right)+\frac{841104}{95} a_{1}\left(\frac{n}{6}\right) \\
& +\frac{1153319904}{8075} a_{1}\left(\frac{n}{12}\right)-\frac{7618}{1425} \Delta_{3,8}(n)-\frac{930778}{1425} \Delta_{3,8}\left(\frac{n}{2}\right)+\frac{19308244}{1425} \Delta_{3,8}\left(\frac{n}{4}\right) \\
& -\frac{29199296}{1425} \Delta_{3,8}\left(\frac{n}{8}\right)+\frac{42968}{855} a_{4}(n)+\frac{93104}{513} a_{4}\left(\frac{n}{2}\right)-\frac{866048}{135} a_{4}\left(\frac{n}{4}\right)-30 a_{2}(n) \\
& -\frac{9882}{19} a_{2}\left(\frac{n}{3}\right)+44 a_{3}(n)-\frac{11664}{19} a_{3}\left(\frac{n}{3}\right)+\frac{214128}{19} a_{5}(n)+\frac{1305792}{19} a_{5}\left(\frac{n}{2}\right) \\
& -224 a_{6}(n)+\frac{56608}{19} a_{6}\left(\frac{n}{2}\right)-\frac{121920}{19} a_{7}(n)+\frac{120448}{19} a_{8}(n)+\frac{170112}{19} a_{9}(n) \text {, } \\
& N\left(1^{7}, 2^{3}, 3,6^{5} ; n\right)=\frac{121}{13382400} \sigma_{7}(n)-\frac{121}{13382400} \sigma_{7}\left(\frac{n}{2}\right)+\frac{1053}{4460800} \sigma_{7}\left(\frac{n}{3}\right)-\frac{121}{836400} \sigma_{7}\left(\frac{n}{4}\right) \\
& -\frac{1053}{4460800} \sigma_{7}\left(\frac{n}{6}\right)+\frac{1936}{52275} \sigma_{7}\left(\frac{n}{8}\right)-\frac{1053}{278800} \sigma_{7}\left(\frac{n}{12}\right)+\frac{16848}{17425} \sigma_{7}\left(\frac{n}{24}\right)-\frac{3131047}{275400} a_{1}(n) \\
& -\frac{52934}{135} a_{1}\left(\frac{n}{2}\right)-\frac{1072557}{3400} a_{1}\left(\frac{n}{3}\right)+\frac{84152864}{34425} a_{1}\left(\frac{n}{4}\right)-\frac{27882}{5} a_{1}\left(\frac{n}{6}\right)+\frac{20993184}{425} a_{1}\left(\frac{n}{12}\right) \\
& +\frac{143657}{36900} \Delta_{3,8}(n)-\frac{3994729}{18450} \Delta_{3,8}\left(\frac{n}{2}\right)-\frac{240190928}{9225} \Delta_{3,8}\left(\frac{n}{4}\right)-\frac{413090368}{9225} \Delta_{3,8}\left(\frac{n}{8}\right) \\
& +\frac{3026}{405} a_{4}(n)+\frac{11584}{27} a_{4}\left(\frac{n}{2}\right)+\frac{9151744}{405} a_{4}\left(\frac{n}{4}\right)-8 a_{2}(n)-918 a_{2}\left(\frac{n}{3}\right)+22 a_{3}(n) \\
& +1404 a_{3}\left(\frac{n}{3}\right)-2556 a_{5}(n)-237696 a_{5}\left(\frac{n}{2}\right)-86 a_{6}(n)-8416 a_{6}\left(\frac{n}{2}\right) \\
& -3520 a_{7}(n)-7744 a_{8}(n)+13696 a_{9}(n) \text {, }
\end{aligned}
$$




$$
\begin{aligned}
& N\left(1^{7}, 2^{3}, 3^{3}, 6^{3} ; n\right)=\frac{121}{6691200} \sigma_{7}(n)-\frac{121}{6691200} \sigma_{7}\left(\frac{n}{2}\right)+\frac{1053}{2230400} \sigma_{7}\left(\frac{n}{3}\right)-\frac{121}{836400} \sigma_{7}\left(\frac{n}{4}\right) \\
& -\frac{1053}{2230400} \sigma_{7}\left(\frac{n}{6}\right)+\frac{1936}{52275} \sigma_{7}\left(\frac{n}{8}\right)-\frac{1053}{278800} \sigma_{7}\left(\frac{n}{12}\right)+\frac{16848}{17425} \sigma_{7}\left(\frac{n}{24}\right)+\frac{123062}{34425} a_{1}(n) \\
& -\frac{57146}{135} a_{1}\left(\frac{n}{2}\right)-\frac{626931}{850} a_{1}\left(\frac{n}{3}\right)+\frac{97023904}{34425} a_{1}\left(\frac{n}{4}\right)-\frac{66078}{5} a_{1}\left(\frac{n}{6}\right)+\frac{12376224}{425} a_{1}\left(\frac{n}{12}\right) \\
& +\frac{335899}{36900} \Delta_{3,8}(n)-\frac{2951993}{18450} \Delta_{3,8}\left(\frac{n}{2}\right)-\frac{391401388}{9225} \Delta_{3,8}\left(\frac{n}{4}\right)-\frac{493830848}{9225} \Delta_{3,8}\left(\frac{n}{8}\right) \\
& -\frac{5138}{405} a_{4}(n)+\frac{18064}{27} a_{4}\left(\frac{n}{2}\right)+\frac{13639424}{405} \Delta_{3,8}\left(\frac{n}{4}\right)-2 a_{2}(n)-1512 a_{2}\left(\frac{n}{3}\right)+16 a_{3}(n) \\
& +2970 a_{3}\left(\frac{n}{3}\right)-10512 a_{5}(n)-356736 a_{5}\left(\frac{n}{2}\right)-60 a_{6}(n)-12896 a_{6}\left(\frac{n}{2}\right)-1376 a_{7}(n) \\
& -12992 a_{8}(n)+123376 a_{9}(n), \\
& N\left(1^{7}, 2^{3}, 3^{5}, 6 ; n\right)=\frac{121}{3345600} \sigma_{7}(n)-\frac{121}{3345600} \sigma_{7}\left(\frac{n}{2}\right)+\frac{1053}{1115200} \sigma_{7}\left(\frac{n}{3}\right)-\frac{121}{836400} \sigma_{7}\left(\frac{n}{4}\right) \\
& -\frac{1053}{1115200} \sigma_{7}\left(\frac{n}{6}\right)+\frac{1936}{52275} \sigma_{7}\left(\frac{n}{8}\right)-\frac{1053}{278800} \sigma_{7}\left(\frac{n}{12}\right)+\frac{16848}{17425} \sigma_{7}\left(\frac{n}{24}\right)+\frac{14188196}{654075} a_{1}(n) \\
& -\frac{89560}{171} a_{1}\left(\frac{n}{2}\right)-\frac{9879849}{8075} a_{1}\left(\frac{n}{3}\right)+\frac{2341964896}{654075} a_{1}\left(\frac{n}{4}\right)-\frac{412740}{19} a_{1}\left(\frac{n}{6}\right)-\frac{177731424}{8075} a_{1}\left(\frac{n}{12}\right) \\
& +\frac{5293121}{350550} \Delta_{3,8}(n)+\frac{1155923}{175275} \Delta_{3,8}\left(\frac{n}{2}\right)-\frac{11797291252}{175275} \Delta_{3,8}\left(\frac{n}{4}\right)-\frac{12320380352}{175275} \Delta_{3,8}\left(\frac{n}{8}\right) \\
& -\frac{283244}{7695} a_{4}(n)+\frac{154576}{171} a_{4}\left(\frac{n}{2}\right)+\frac{21035264}{405} a_{4}\left(\frac{n}{4}\right)+10 a_{2}(n)-\frac{34074}{19} a_{2}\left(\frac{n}{3}\right)+4 a_{3}(n) \\
& +\frac{80244}{19} a_{3}\left(\frac{n}{3}\right)-\frac{368208}{19} a_{5}(n)-\frac{10443072}{19} a_{5}\left(\frac{n}{2}\right)-8 a_{6}(n)-\frac{380384}{19} a_{6}\left(\frac{n}{2}\right)-\frac{512}{19} a_{7}(n) \\
& -\frac{411392}{19} a_{8}(n)+\frac{316928}{19} a_{9}(n) \text {, } \\
& N\left(1^{7}, 2^{5}, 3,6^{3} ; n\right)=\frac{73}{2676480} \sigma_{7}(n)-\frac{73}{2676480} \sigma_{7}\left(\frac{n}{2}\right)-\frac{243}{892160} \sigma_{7}\left(\frac{n}{3}\right)+\frac{73}{167280} \sigma_{7}\left(\frac{n}{4}\right) \\
& +\frac{243}{892160} \sigma_{7}\left(\frac{n}{6}\right)-\frac{1168}{10455} \sigma_{7}\left(\frac{n}{8}\right)-\frac{243}{55760} \sigma_{7}\left(\frac{n}{12}\right)+\frac{3888}{3485} \sigma_{7}\left(\frac{n}{24}\right)-\frac{9429853}{209304} a_{1}(n) \\
& -\frac{278462}{855} a_{1}\left(\frac{n}{2}\right)+\frac{1440441}{2584} a_{1}\left(\frac{n}{3}\right)+\frac{223785376}{130815} a_{1}\left(\frac{n}{4}\right)+\frac{941202}{95} a_{1}\left(\frac{n}{6}\right)+\frac{185425056}{1615} a_{1}\left(\frac{n}{12}\right) \\
& -\frac{192955}{28044} \Delta_{3,8}(n)-\frac{30421301}{70110} \Delta_{3,8}\left(\frac{n}{2}\right)+\frac{69539080}{7011} \Delta_{3,8}\left(\frac{n}{4}\right)-\frac{938645312}{35055} \Delta_{3,8}\left(\frac{n}{8}\right) \\
& +\frac{79906}{1539} a_{4}(n)-\frac{416}{171} a_{4}\left(\frac{n}{2}\right)-\frac{175360}{81} a_{4}\left(\frac{n}{4}\right)-24 a_{2}(n)+\frac{54}{19} a_{2}\left(\frac{n}{3}\right)+38 a_{3}(n) \\
& -\frac{27756}{19} a_{3}\left(\frac{n}{3}\right)+\frac{260316}{19} a_{5}(n)+\frac{512256}{19} a_{5}\left(\frac{n}{2}\right)-166 a_{6}(n)+\frac{28064}{19} a_{6}\left(\frac{n}{2}\right) \\
& -\frac{145792}{19} a_{7}(n)+\frac{80960}{19} a_{8}(n)+\frac{263680}{19} a_{9}(n) \text {, }
\end{aligned}
$$




$$
\begin{aligned}
& N\left(1^{7}, 2^{5}, 3^{3}, 6 ; n\right)=\frac{73}{1338240} \sigma_{7}(n)-\frac{73}{1338240} \sigma_{7}\left(\frac{n}{2}\right)-\frac{243}{446080} \sigma_{7}\left(\frac{n}{3}\right)+\frac{73}{167280} \sigma_{7}\left(\frac{n}{4}\right) \\
& +\frac{243}{446080} \sigma_{7}\left(\frac{n}{6}\right)-\frac{1168}{10455} \sigma_{7}\left(\frac{n}{8}\right)-\frac{243}{55760} \sigma_{7}\left(\frac{n}{12}\right)+\frac{3888}{3485} \sigma_{7}\left(\frac{n}{24}\right)-\frac{3662272}{43605} a_{1}(n) \\
& -\frac{47210}{171} a_{1}\left(\frac{n}{2}\right)+\frac{5646483}{3230} a_{1}\left(\frac{n}{3}\right)+\frac{35369824}{43605} a_{1}\left(\frac{n}{4}\right)+\frac{586062}{19} a_{1}\left(\frac{n}{6}\right)+\frac{218992032}{1615} a_{1}\left(\frac{n}{12}\right) \\
& -\frac{1008589}{46740} \Delta_{3,8}(n)-\frac{12542377}{23370} \Delta_{3,8}\left(\frac{n}{2}\right)+\frac{597244972}{11685} \Delta_{3,8}\left(\frac{n}{4}\right)-\frac{64579648}{11685} \Delta_{3,8}\left(\frac{n}{8}\right) \\
& +\frac{54142}{513} a_{4}(n)-\frac{114640}{171} a_{4}\left(\frac{n}{2}\right)-\frac{798976}{27} a_{4}\left(\frac{n}{4}\right)-34 a_{2}(n)+\frac{40176}{19} a_{2}\left(\frac{n}{3}\right)+48 a_{3}(n) \\
& -\frac{123282}{19} a_{3}\left(\frac{n}{3}\right)+\frac{663984}{19} a_{5}(n)+\frac{6177024}{19} a_{5}\left(\frac{n}{2}\right)-220 a_{6}(n)+\frac{238624}{19} a_{6}\left(\frac{n}{2}\right) \\
& -\frac{271392}{19} a_{7}(n)+\frac{315840}{19} a_{8}(n)+\frac{335040}{19} a_{9}(n) \text {, } \\
& N\left(1^{7}, 2^{7}, 3,6 ; n\right)=\frac{1093}{13382400} \sigma_{7}(n)-\frac{1093}{13382400} \sigma_{7}\left(\frac{n}{2}\right)+\frac{729}{4460800} \sigma_{7}\left(\frac{n}{3}\right)-\frac{1093}{836400} \sigma_{7}\left(\frac{n}{4}\right) \\
& -\frac{729}{4460800} \sigma_{7}\left(\frac{n}{6}\right)+\frac{17488}{52275} \sigma_{7}\left(\frac{n}{8}\right)-\frac{729}{278800} \sigma_{7}\left(\frac{n}{12}\right)+\frac{11664}{17425} \sigma_{7}\left(\frac{n}{24}\right)+\frac{25038359}{581400} a_{1}(n) \\
& -\frac{31966}{171} a_{1}\left(\frac{n}{2}\right)-\frac{114249339}{64600} a_{1}\left(\frac{n}{3}\right)+\frac{149981152}{72675} a_{1}\left(\frac{n}{4}\right)-\frac{584550}{19} a_{1}\left(\frac{n}{6}\right)+\frac{417914208}{8075} a_{1}\left(\frac{n}{12}\right) \\
& +\frac{5102413}{233700} \Delta_{3,8}(n)-\frac{17351981}{116850} \Delta_{3,8}\left(\frac{n}{2}\right)-\frac{3466321072}{58425} \Delta_{3,8}\left(\frac{n}{4}\right)-\frac{2526270272}{58425} \Delta_{3,8}\left(\frac{n}{8}\right) \\
& -\frac{55522}{855} a_{4}(n)+\frac{201664}{171} a_{4}\left(\frac{n}{2}\right)+\frac{1810688}{45} a_{4}\left(\frac{n}{4}\right)-\frac{75978}{19} a_{2}\left(\frac{n}{3}\right)+14 a_{3}(n)+\frac{159084}{19} a_{3}\left(\frac{n}{3}\right) \\
& -\frac{565380}{19} a_{5}(n)-\frac{8355456}{19} a_{5}\left(\frac{n}{2}\right)-14 a_{6}(n)-\frac{307744}{19} a_{6}\left(\frac{n}{2}\right)+\frac{159168}{19} a_{7}(n)-\frac{312000}{19} a_{8}(n) \\
& -\frac{61824}{19} a_{9}(n) \text {, } \\
& N\left(1^{8}, 6^{8} ; n\right)=\frac{1}{334560} \sigma_{7}(n)-\frac{1}{334560} \sigma_{7}\left(\frac{n}{2}\right)+\frac{27}{111520} \sigma_{7}\left(\frac{n}{3}\right)-\frac{1}{20910} \sigma_{7}\left(\frac{n}{4}\right) \\
& -\frac{27}{111520} \sigma_{7}\left(\frac{n}{6}\right)+\frac{128}{10455} \sigma_{7}\left(\frac{n}{8}\right)-\frac{27}{6970} \sigma_{7}\left(\frac{n}{12}\right)+\frac{3456}{3485} \sigma_{7}\left(\frac{n}{24}\right)-\frac{336557}{14535} a_{1}(n) \\
& -\frac{988048}{2565} a_{1}\left(\frac{n}{2}\right)-\frac{103653}{1615} a_{1}\left(\frac{n}{3}\right)+\frac{105769216}{43605} a_{1}\left(\frac{n}{4}\right)-\frac{95904}{95} a_{1}\left(\frac{n}{6}\right)+\frac{143140608}{1615} a_{1}\left(\frac{n}{12}\right) \\
& +\frac{9242}{11685} \Delta_{3,8}(n)-\frac{3213556}{11685} \Delta_{3,8}\left(\frac{n}{2}\right)-\frac{12286592}{779} \Delta_{3,8}\left(\frac{n}{4}\right)-\frac{166772224}{3895} \Delta_{3,8}\left(\frac{n}{8}\right) \\
& +\frac{3824}{171} a_{4}(n)+\frac{151040}{513} a_{4}\left(\frac{n}{2}\right)+\frac{432128}{27} a_{4}\left(\frac{n}{4}\right)-16 a_{2}(n)-\frac{16848}{19} a_{2}\left(\frac{n}{3}\right)+32 a_{3}(n) \\
& +\frac{16848}{19} a_{3}\left(\frac{n}{3}\right)+\frac{51552}{19} a_{5}(n)-\frac{3193344}{19} a_{5}\left(\frac{n}{2}\right)-112 a_{6}(n)-\frac{107776}{19} a_{6}\left(\frac{n}{2}\right) \\
& -\frac{98304}{19} a_{7}(n)-\frac{73728}{19} a_{8}(n)+\frac{264192}{19} a_{9}(n) \text {, }
\end{aligned}
$$




$$
\begin{aligned}
& N\left(1^{8}, 3^{2}, 6^{6} ; n\right)=\frac{1}{167280} \sigma_{7}(n)-\frac{1}{167280} \sigma_{7}\left(\frac{n}{2}\right)+\frac{27}{55760} \sigma_{7}\left(\frac{n}{3}\right)-\frac{1}{20910} \sigma_{7}\left(\frac{n}{4}\right) \\
& -\frac{27}{55760} \sigma_{7}\left(\frac{n}{6}\right)+\frac{128}{10455} \sigma_{7}\left(\frac{n}{8}\right)-\frac{27}{6970} \sigma_{7}\left(\frac{n}{12}\right)+\frac{3456}{3485} \sigma_{7}\left(\frac{n}{24}\right)-\frac{269092}{14535} a_{1}(n) \\
& -\frac{319952}{855} a_{1}\left(\frac{n}{2}\right)-\frac{279828}{1615} a_{1}\left(\frac{n}{3}\right)+\frac{35993344}{14535} a_{1}\left(\frac{n}{4}\right)-\frac{321408}{95} a_{1}\left(\frac{n}{6}\right)+\frac{148075776}{1615} a_{1}\left(\frac{n}{12}\right) \\
& +\frac{24962}{11685} \Delta_{3,8}(n)-\frac{2739244}{11685} \Delta_{3,8}\left(\frac{n}{2}\right)-\frac{14751840}{779} \Delta_{3,8}\left(\frac{n}{4}\right)-\frac{171852288}{3895} \Delta_{3,8}\left(\frac{n}{8}\right) \\
& +\frac{2800}{171} a_{4}(n)+\frac{56704}{171} a_{4}\left(\frac{n}{2}\right)+\frac{161792}{9} a_{4}\left(\frac{n}{4}\right)-16 a_{2}(n)-\frac{22356}{19} a_{2}\left(\frac{n}{3}\right)+32 a_{3}(n) \\
& +\frac{28512}{19} a_{3}\left(\frac{n}{3}\right)+\frac{20736}{19} a_{5}(n)-\frac{3612672}{19} a_{5}\left(\frac{n}{2}\right)-96 a_{6}(n)-\frac{123648}{19} a_{6}\left(\frac{n}{2}\right) \\
& -\frac{82176}{19} a_{7}(n)-\frac{89600}{19} a_{8}(n)+\frac{247296}{19} a_{9}(n) \text {, } \\
& N\left(1^{8}, 3^{4}, 6^{4} ; n\right)=\frac{1}{83640} \sigma_{7}(n)-\frac{1}{83640} \sigma_{7}\left(\frac{n}{2}\right)+\frac{27}{27880} \sigma_{7}\left(\frac{n}{3}\right)-\frac{1}{20910} \sigma_{7}\left(\frac{n}{4}\right) \\
& -\frac{27}{27880} \sigma_{7}\left(\frac{n}{6}\right)+\frac{128}{10455} \sigma_{7}\left(\frac{n}{8}\right)-\frac{27}{6970} \sigma_{7}\left(\frac{n}{12}\right)+\frac{3456}{3485} \sigma_{7}\left(\frac{n}{24}\right)-\frac{574424}{43605} a_{1}(n) \\
& -\frac{101728}{285} a_{1}\left(\frac{n}{2}\right)-\frac{442152}{1615} a_{1}\left(\frac{n}{3}\right)+\frac{110190848}{43605} a_{1}\left(\frac{n}{4}\right)-\frac{489456}{95} a_{1}\left(\frac{n}{6}\right)+\frac{153010944}{1615} a_{1}\left(\frac{n}{12}\right) \\
& +\frac{39428}{11685} \Delta_{3,8}(n)-\frac{529688}{3895} \Delta_{3,8}\left(\frac{n}{2}\right)-\frac{256287008}{11685} \Delta_{3,8}\left(\frac{n}{4}\right)-\frac{176932352}{3895} \Delta_{3,8}\left(\frac{n}{8}\right) \\
& +\frac{5024}{513} a_{4}(n)+\frac{6016}{19} a_{4}\left(\frac{n}{2}\right)+\frac{538624}{27} a_{4}\left(\frac{n}{4}\right)-16 a_{2}(n)-\frac{27864}{19} a_{2}\left(\frac{n}{3}\right)+32 a_{3}(n) \\
& +\frac{40176}{19} a_{3}\left(\frac{n}{3}\right)-\frac{4608}{19} a_{5}(n)-\frac{4032000}{19} a_{5}\left(\frac{n}{2}\right)-64 a_{6}(n)-\frac{139520}{19} a_{6}\left(\frac{n}{2}\right) \\
& -\frac{66048}{19} a_{7}(n)-\frac{105472}{19} a_{8}(n)+\frac{230400}{19} a_{9}(n) \text {, } \\
& N\left(1^{8}, 3^{6}, 6^{2} ; n\right)=\frac{1}{41820} \sigma_{7}(n)-\frac{1}{41820} \sigma_{7}\left(\frac{n}{2}\right)+\frac{27}{13940} \sigma_{7}\left(\frac{n}{3}\right)-\frac{1}{20910} \sigma_{7}\left(\frac{n}{4}\right) \\
& -\frac{27}{13940} \sigma_{7}\left(\frac{n}{6}\right)+\frac{128}{10455} \sigma_{7}\left(\frac{n}{8}\right)-\frac{27}{6970} \sigma_{7}\left(\frac{n}{12}\right)+\frac{3456}{3485} \sigma_{7}\left(\frac{n}{24}\right)-\frac{378952}{43605} a_{1}(n) \\
& -\frac{843808}{2565} a_{1}\left(\frac{n}{2}\right)-\frac{107676}{1615} a_{1}\left(\frac{n}{3}\right)+\frac{110190848}{43605} a_{1}\left(\frac{n}{4}\right)-\frac{292464}{95} a_{1}\left(\frac{n}{6}\right)+\frac{153010944}{1615} a_{1}\left(\frac{n}{12}\right) \\
& +\frac{56224}{11685} \Delta_{3,8}(n)+\frac{858896}{11685} \Delta_{3,8}\left(\frac{n}{2}\right)-\frac{249107744}{11685} \Delta_{3,8}\left(\frac{n}{4}\right)-\frac{176932352}{3895} \Delta_{3,8}\left(\frac{n}{8}\right) \\
& +\frac{1984}{513} a_{4}(n)+\frac{84608}{513} a_{4}\left(\frac{n}{2}\right)+\frac{538624}{27} a_{4}\left(\frac{n}{4}\right)-16 a_{2}(n)-\frac{27864}{19} a_{2}\left(\frac{n}{3}\right)+32 a_{3}(n) \\
& +\frac{40176}{19} a_{3}\left(\frac{n}{3}\right)+\frac{17280}{19} a_{5}(n)-\frac{4032000}{19} a_{5}\left(\frac{n}{2}\right)-\frac{139520}{19} a_{6}\left(\frac{n}{2}\right)-\frac{66048}{19} a_{7}(n) \\
& -\frac{105472}{19} a_{8}(n)+\frac{230400}{19} a_{9}(n) \text {, }
\end{aligned}
$$




$$
\begin{aligned}
& N\left(1^{8}, 3^{8} ; n\right)=\frac{1}{20910} \sigma_{7}(n)-\frac{1}{10455} \sigma_{7}\left(\frac{n}{2}\right)+\frac{27}{6970} \sigma_{7}\left(\frac{n}{3}\right)+\frac{128}{10455} \sigma_{7}\left(\frac{n}{4}\right) \\
& -\frac{27}{3485} \sigma_{7}\left(\frac{n}{6}\right)+\frac{3456}{3485} \sigma_{7}\left(\frac{n}{12}\right)+\frac{1792}{255} a_{1}(n)+\frac{28672}{255} a_{1}\left(\frac{n}{2}\right)+\frac{48384}{85} a_{1}\left(\frac{n}{3}\right) \\
& +\frac{774144}{85} a_{1}\left(\frac{n}{6}\right)+\frac{5504}{615} \Delta_{3,8}(n)-\frac{512}{615} \Delta_{3,8}\left(\frac{n}{2}\right)+\frac{1409024}{615} \Delta_{3,8}\left(\frac{n}{4}\right), \\
& N\left(1^{8}, 2^{2}, 6^{6} ; n\right)=\frac{61}{6691200} \sigma_{7}(n)-\frac{61}{6691200} \sigma_{7}\left(\frac{n}{2}\right)-\frac{567}{2230400} \sigma_{7}\left(\frac{n}{3}\right) \\
& +\frac{61}{418200} \sigma_{7}\left(\frac{n}{4}\right)+\frac{567}{2230400} \sigma_{7}\left(\frac{n}{6}\right)-\frac{1952}{52275} \sigma_{7}\left(\frac{n}{8}\right)-\frac{567}{139400} \sigma_{7}\left(\frac{n}{12}\right) \\
& +\frac{18144}{17425} \sigma_{7}\left(\frac{n}{24}\right)-\frac{70547473}{2616300} a_{1}(n)-\frac{856904}{2565} a_{1}\left(\frac{n}{2}\right)-\frac{591363}{32300} a_{1}\left(\frac{n}{3}\right) \\
& +\frac{1323512128}{654075} a_{1}\left(\frac{n}{4}\right)-\frac{55872}{95} a_{1}\left(\frac{n}{6}\right)+\frac{919205568}{8075} a_{1}\left(\frac{n}{12}\right) \\
& +\frac{79763}{350550} \Delta_{3,8}(n)-\frac{73394191}{175275} \Delta_{3,8}\left(\frac{n}{2}\right)-\frac{1376658496}{175275} \Delta_{3,8}\left(\frac{n}{4}\right) \\
& -\frac{6073751936}{175275} \Delta_{3,8}\left(\frac{n}{8}\right)+\frac{205708}{7695} a_{4}(n)+\frac{172288}{513} a_{4}\left(\frac{n}{2}\right) \\
& +\frac{3717632}{405} a_{4}\left(\frac{n}{4}\right)-20 a_{2}(n)-\frac{21924}{19} a_{2}\left(\frac{n}{3}\right)+36 a_{3}(n) \\
& +\frac{21924}{19} a_{3}\left(\frac{n}{3}\right)+\frac{71784}{19} a_{5}(n)-\frac{1856640}{19} a_{5}\left(\frac{n}{2}\right)-148 a_{6}(n) \\
& -\frac{60864}{19} a_{6}\left(\frac{n}{2}\right)-\frac{81152}{19} a_{7}(n)-\frac{15872}{19} a_{8}(n)+\frac{197120}{19} a_{9}(n) \text {, } \\
& N\left(1^{8}, 2^{2}, 3^{2}, 6^{4} ; n\right)=\frac{61}{3345600} \sigma_{7}(n)-\frac{61}{3345600} \sigma_{7}\left(\frac{n}{2}\right)-\frac{567}{1115200} \sigma_{7}\left(\frac{n}{3}\right) \\
& +\frac{61}{418200} \sigma_{7}\left(\frac{n}{4}\right)+\frac{567}{1115200} \sigma_{7}\left(\frac{n}{6}\right)-\frac{1952}{52275} \sigma_{7}\left(\frac{n}{8}\right)-\frac{567}{139400} \sigma_{7}\left(\frac{n}{12}\right) \\
& +\frac{18144}{17425} \sigma_{7}\left(\frac{n}{24}\right)-\frac{1415906}{34425} a_{1}(n)-\frac{48164}{135} a_{1}\left(\frac{n}{2}\right)+\frac{159039}{425} a_{1}\left(\frac{n}{3}\right) \\
& +\frac{68261312}{34425} a_{1}\left(\frac{n}{4}\right)+\frac{32868}{5} a_{1}\left(\frac{n}{6}\right)+\frac{51291072}{425} a_{1}\left(\frac{n}{12}\right)-\frac{85181}{18450} \Delta_{3,8}(n) \\
& -\frac{4047713}{9225} \Delta_{3,8}\left(\frac{n}{2}\right)+\frac{27941896}{9225} \Delta_{3,8}\left(\frac{n}{4}\right)-\frac{298927744}{9225} \Delta_{3,8}\left(\frac{n}{8}\right) \\
& +\frac{18524}{405} a_{4}(n)+\frac{3232}{27} a_{4}\left(\frac{n}{2}\right)+\frac{1126912}{405} a_{4}\left(\frac{n}{4}\right)-24 a_{2}(n)-432 a_{2}\left(\frac{n}{3}\right) \\
& +40 a_{3}(n)-540 a_{3}\left(\frac{n}{3}\right)+10944 a_{5}(n)-26880 a_{5}\left(\frac{n}{2}\right)-168 a_{6}(n) \\
& -448 a_{6}\left(\frac{n}{2}\right)-6976 a_{7}(n)+2432 a_{8}(n)+13696 a_{9}(n),
\end{aligned}
$$




$$
\begin{aligned}
& N\left(1^{8}, 2^{2}, 3^{4}, 6^{2} ; n\right)=\frac{61}{1672800} \sigma_{7}(n)-\frac{61}{1672800} \sigma_{7}\left(\frac{n}{2}\right)-\frac{567}{557600} \sigma_{7}\left(\frac{n}{3}\right)+\frac{61}{418200} \sigma_{7}\left(\frac{n}{4}\right) \\
& +\frac{567}{557600} \sigma_{7}\left(\frac{n}{6}\right)-\frac{1952}{52275} \sigma_{7}\left(\frac{n}{8}\right)-\frac{567}{139400} \sigma_{7}\left(\frac{n}{12}\right)+\frac{18144}{17425} \sigma_{7}\left(\frac{n}{24}\right)-\frac{37093598}{654075} a_{1}(n) \\
& -\frac{16576}{57} a_{1}\left(\frac{n}{2}\right)+\frac{6549912}{8075} a_{1}\left(\frac{n}{3}\right)+\frac{931462208}{654075} a_{1}\left(\frac{n}{4}\right)+\frac{276120}{19} a_{1}\left(\frac{n}{6}\right)+\frac{1225107648}{8075} a_{1}\left(\frac{n}{12}\right) \\
& -\frac{1754149}{175275} \Delta_{3,8}(n)-\frac{97580774}{175275} \Delta_{3,8}\left(\frac{n}{2}\right)+\frac{3995678104}{175275} \Delta_{3,8}\left(\frac{n}{4}\right)-\frac{3492628096}{175275} \Delta_{3,8}\left(\frac{n}{8}\right) \\
& +\frac{513272}{7695} a_{4}(n)-\frac{1888}{19} a_{4}\left(\frac{n}{2}\right)-\frac{4602368}{405} a_{4}\left(\frac{n}{4}\right)-32 a_{2}(n)+\frac{864}{19} a_{2}\left(\frac{n}{3}\right)+48 a_{3}(n) \\
& -\frac{37800}{19} a_{3}\left(\frac{n}{3}\right)+\frac{356544}{19} a_{5}(n)+\frac{2352000}{19} a_{5}\left(\frac{n}{2}\right)-208 a_{6}(n)+\frac{97600}{19} a_{6}\left(\frac{n}{2}\right) \\
& -\frac{165760}{19} a_{7}(n)+\frac{171776}{19} a_{8}(n)+\frac{235264}{19} a_{9}(n) \text {, } \\
& N\left(1^{8}, 2^{2}, 3^{6} ; n\right)=\frac{61}{836400} \sigma_{7}(n)-\frac{61}{836400} \sigma_{7}\left(\frac{n}{2}\right)-\frac{567}{278800} \sigma_{7}\left(\frac{n}{3}\right)+\frac{61}{418200} \sigma_{7}\left(\frac{n}{4}\right) \\
& +\frac{567}{278800} \sigma_{7}\left(\frac{n}{6}\right)-\frac{1952}{52275} \sigma_{7}\left(\frac{n}{8}\right)-\frac{567}{139400} \sigma_{7}\left(\frac{n}{12}\right)+\frac{18144}{17425} \sigma_{7}\left(\frac{n}{24}\right)-\frac{16341662}{218025} a_{1}(n) \\
& -\frac{356072}{2565} a_{1}\left(\frac{n}{2}\right)+\frac{10050534}{8075} a_{1}\left(\frac{n}{3}\right)+\frac{17118272}{72675} a_{1}\left(\frac{n}{4}\right)+\frac{2171664}{95} a_{1}\left(\frac{n}{6}\right)+\frac{1541585088}{8075} a_{1}\left(\frac{n}{12}\right) \\
& -\frac{897056}{58425} \Delta_{3,8}(n)-\frac{54842116}{58425} \Delta_{3,8}\left(\frac{n}{2}\right)+\frac{2954451848}{58425} \Delta_{3,8}\left(\frac{n}{4}\right)+\frac{259643008}{58425} \Delta_{3,8}\left(\frac{n}{8}\right) \\
& +\frac{241808}{2565} a_{4}(n)-\frac{92960}{513} a_{4}\left(\frac{n}{2}\right)-\frac{1475072}{45} a_{4}\left(\frac{n}{4}\right)-44 a_{2}(n)+\frac{8100}{19} a_{2}\left(\frac{n}{3}\right)+56 a_{3}(n) \\
& -\frac{63504}{19} a_{3}\left(\frac{n}{3}\right)+\frac{498528}{19} a_{5}(n)+\frac{6643584}{19} a_{5}\left(\frac{n}{2}\right)-320 a_{6}(n)+\frac{252736}{19} a_{6}\left(\frac{n}{2}\right) \\
& -\frac{177792}{19} a_{7}(n)+\frac{346368}{19} a_{8}(n)+\frac{109824}{19} a_{9}(n) \text {, } \\
& N\left(1^{8}, 2^{4}, 6^{4} ; n\right)=\frac{91}{3345600} \sigma_{7}(n)-\frac{91}{3345600} \sigma_{7}\left(\frac{n}{2}\right)+\frac{243}{1115200} \sigma_{7}\left(\frac{n}{3}\right)-\frac{91}{209100} \sigma_{7}\left(\frac{n}{4}\right) \\
& -\frac{243}{1115200} \sigma_{7}\left(\frac{n}{6}\right)+\frac{5824}{52275} \sigma_{7}\left(\frac{n}{8}\right)-\frac{243}{69700} \sigma_{7}\left(\frac{n}{12}\right)+\frac{15552}{17425} \sigma_{7}\left(\frac{n}{24}\right)-\frac{8991121}{436050} a_{1}(n) \\
& -\frac{420592}{855} a_{1}\left(\frac{n}{2}\right)-\frac{1788453}{16150} a_{1}\left(\frac{n}{3}\right)+\frac{624003968}{218025} a_{1}\left(\frac{n}{4}\right)-\frac{228528}{95} a_{1}\left(\frac{n}{6}\right)+\frac{347082624}{8075} a_{1}\left(\frac{n}{12}\right) \\
& +\frac{79801}{58425} \Delta_{3,8}(n)-\frac{9330754}{58425} \Delta_{3,8}\left(\frac{n}{2}\right)-\frac{1535303296}{58425} \Delta_{3,8}\left(\frac{n}{4}\right)-\frac{3042319616}{58425} \Delta_{3,8}\left(\frac{n}{8}\right) \\
& +\frac{49352}{2565} a_{4}(n)+\frac{52736}{171} a_{4}\left(\frac{n}{2}\right)+\frac{3361792}{135} a_{4}\left(\frac{n}{4}\right)-8 a_{2}(n)-\frac{8424}{19} a_{2}\left(\frac{n}{3}\right)+24 a_{3}(n) \\
& +\frac{8424}{19} a_{3}\left(\frac{n}{3}\right)+\frac{36720}{19} a_{5}(n)-\frac{4923648}{19} a_{5}\left(\frac{n}{2}\right)-88 a_{6}(n)-\frac{170624}{19} a_{6}\left(\frac{n}{2}\right) \\
& -\frac{107520}{19} a_{7}(n)-\frac{153600}{19} a_{8}(n)+\frac{365568}{19} a_{9}(n) \text {, }
\end{aligned}
$$




$$
\begin{aligned}
& N\left(1^{8}, 2^{4}, 3^{2}, 6^{2} ; n\right)=\frac{91}{1672800} \sigma_{7}(n)-\frac{91}{1672800} \sigma_{7}\left(\frac{n}{2}\right)+\frac{243}{557600} \sigma_{7}\left(\frac{n}{3}\right)-\frac{91}{209100} \sigma_{7}\left(\frac{n}{4}\right) \\
& -\frac{243}{557600} \sigma_{7}\left(\frac{n}{6}\right)+\frac{5824}{52275} \sigma_{7}\left(\frac{n}{8}\right)-\frac{243}{69700} \sigma_{7}\left(\frac{n}{12}\right)+\frac{15552}{17425} \sigma_{7}\left(\frac{n}{24}\right)+\frac{12888152}{654075} a_{1}(n) \\
& -\frac{1087832}{2565} a_{1}\left(\frac{n}{2}\right)-\frac{10271538}{8075} a_{1}\left(\frac{n}{3}\right)+\frac{2024593024}{654075} a_{1}\left(\frac{n}{4}\right)-\frac{2191896}{95} a_{1}\left(\frac{n}{6}\right)+\frac{310460544}{8075} a_{1}\left(\frac{n}{12}\right) \\
& +\frac{2752051}{175275} \Delta_{3,8}(n)-\frac{27900754}{175275} \Delta_{3,8}\left(\frac{n}{2}\right)-\frac{10196115568}{175275} \Delta_{3,8}\left(\frac{n}{4}\right)-\frac{10601017088}{175275} \Delta_{3,8}\left(\frac{n}{8}\right) \\
& -\frac{272648}{7695} a_{4}(n)+\frac{507712}{513} a_{4}\left(\frac{n}{2}\right)+\frac{17662976}{405} a_{4}\left(\frac{n}{4}\right)-\frac{52380}{19} a_{2}\left(\frac{n}{3}\right)+16 a_{3}(n) \\
& +\frac{107784}{19} a_{3}\left(\frac{n}{3}\right)-\frac{377280}{19} a_{5}(n)-\frac{8873472}{19} a_{5}\left(\frac{n}{2}\right)-48 a_{6}(n)-\frac{322944}{19} a_{6}\left(\frac{n}{2}\right) \\
& +\frac{43648}{19} a_{7}(n)-\frac{325376}{19} a_{8}(n)+\frac{188672}{19} a_{9}(n) \text {, } \\
& N\left(1^{8}, 2^{4}, 3^{4} ; n\right)=\frac{91}{836400} \sigma_{7}(n)-\frac{91}{836400} \sigma_{7}\left(\frac{n}{2}\right)+\frac{243}{278800} \sigma_{7}\left(\frac{n}{3}\right)-\frac{91}{209100} \sigma_{7}\left(\frac{n}{4}\right) \\
& -\frac{243}{278800} \sigma_{7}\left(\frac{n}{6}\right)+\frac{5824}{52275} \sigma_{7}\left(\frac{n}{8}\right)-\frac{243}{69700} \sigma_{7}\left(\frac{n}{12}\right)+\frac{15552}{17425} \sigma_{7}\left(\frac{n}{24}\right)+\frac{39930844}{654075} a_{1}(n) \\
& -\frac{1555168}{2565} a_{1}\left(\frac{n}{2}\right)-\frac{20790936}{8075} a_{1}\left(\frac{n}{3}\right)+\frac{3095663744}{654075} a_{1}\left(\frac{n}{4}\right)-\frac{4328064}{95} a_{1}\left(\frac{n}{6}\right)-\frac{155051136}{8075} a_{1}\left(\frac{n}{12}\right) \\
& +\frac{5570522}{175275} \Delta_{3,8}(n)+\frac{13068652}{175275} \Delta_{3,8}\left(\frac{n}{2}\right)-\frac{19757597648}{175275} \Delta_{3,8}\left(\frac{n}{4}\right)-\frac{16900611328}{175275} \Delta_{3,8}\left(\frac{n}{8}\right) \\
& -\frac{714736}{7695} a_{4}(n)+\frac{861632}{513} a_{4}\left(\frac{n}{2}\right)+\frac{33442816}{405} a_{4}\left(\frac{n}{4}\right)+16 a_{2}(n)-\frac{85320}{19} a_{2}\left(\frac{n}{3}\right)+\frac{196128}{19} a_{3}\left(\frac{n}{3}\right) \\
& -\frac{792576}{19} a_{5}(n)-\frac{16800000}{19} a_{5}\left(\frac{n}{2}\right)+32 a_{6}(n)-\frac{613760}{19} a_{6}\left(\frac{n}{2}\right)+\frac{133376}{19} a_{7}(n) \\
& -\frac{659968}{19} a_{8}(n)+\frac{220672}{19} a_{9}(n) \text {, } \\
& N\left(1^{8}, 2^{6}, 6^{2} ; n\right)=\frac{547}{6691200} \sigma_{7}(n)-\frac{547}{6691200} \sigma_{7}\left(\frac{n}{2}\right)-\frac{729}{2230400} \sigma_{7}\left(\frac{n}{3}\right)+\frac{547}{418200} \sigma_{7}\left(\frac{n}{4}\right) \\
& +\frac{729}{2230400} \sigma_{7}\left(\frac{n}{6}\right)-\frac{17504}{52275} \sigma_{7}\left(\frac{n}{8}\right)-\frac{729}{139400} \sigma_{7}\left(\frac{n}{12}\right)+\frac{23328}{17425} \sigma_{7}\left(\frac{n}{24}\right)-\frac{10045919}{290700} a_{1}(n) \\
& -\frac{836296}{2565} a_{1}\left(\frac{n}{2}\right)+\frac{1261899}{32300} a_{1}\left(\frac{n}{3}\right)+\frac{447252032}{218025} a_{1}\left(\frac{n}{4}\right)-\frac{16848}{95} a_{1}\left(\frac{n}{6}\right)+\frac{1320826176}{8075} a_{1}\left(\frac{n}{12}\right) \\
& -\frac{18711}{38950} \Delta_{3,8}(n)-\frac{12219653}{19475} \Delta_{3,8}\left(\frac{n}{2}\right)-\frac{29382688}{19475} \Delta_{3,8}\left(\frac{n}{4}\right)-\frac{655449728}{19475} \Delta_{3,8}\left(\frac{n}{8}\right) \\
& +\frac{29924}{855} a_{4}(n)+\frac{222080}{513} a_{4}\left(\frac{n}{2}\right)+\frac{537088}{135} a_{4}\left(\frac{n}{4}\right)-28 a_{2}(n)-\frac{32076}{19} a_{2}\left(\frac{n}{3}\right)+44 a_{3}(n) \\
& +\frac{32076}{19} a_{3}\left(\frac{n}{3}\right)+\frac{84888}{19} a_{5}(n)-\frac{846720}{19} a_{5}\left(\frac{n}{2}\right)-204 a_{6}(n)-\frac{25408}{19} a_{6}\left(\frac{n}{2}\right) \\
& -\frac{76032}{19} a_{7}(n)+\frac{41472}{19} a_{8}(n)+\frac{179712}{19} a_{9}(n) \text {, }
\end{aligned}
$$




$$
\begin{aligned}
& N\left(1^{8}, 2^{6}, 3^{2} ; n\right)=\frac{547}{3345600} \sigma_{7}(n)-\frac{547}{3345600} \sigma_{7}\left(\frac{n}{2}\right)-\frac{729}{1115200} \sigma_{7}\left(\frac{n}{3}\right) \\
& +\frac{547}{418200} \sigma_{7}\left(\frac{n}{4}\right)+\frac{729}{1115200} \sigma_{7}\left(\frac{n}{6}\right)-\frac{17504}{52275} \sigma_{7}\left(\frac{n}{8}\right)-\frac{729}{139400} \sigma_{7}\left(\frac{n}{12}\right) \\
& +\frac{23328}{17425} \sigma_{7}\left(\frac{n}{24}\right)-\frac{31348886}{218025} a_{1}(n)-\frac{1190372}{2565} a_{1}\left(\frac{n}{2}\right)+\frac{27469827}{8075} a_{1}\left(\frac{n}{3}\right) \\
& +\frac{73910464}{72675} a_{1}\left(\frac{n}{4}\right)+\frac{5763204}{95} a_{1}\left(\frac{n}{6}\right)+\frac{1042635456}{8075} a_{1}\left(\frac{n}{12}\right) \\
& -\frac{4907011}{116850} \Delta_{3,8}(n)-\frac{30718303}{58425} \Delta_{3,8}\left(\frac{n}{2}\right)+\frac{5292946136}{58425} \Delta_{3,8}\left(\frac{n}{4}\right) \\
& -\frac{200607104}{58425} \Delta_{3,8}\left(\frac{n}{8}\right)+\frac{476324}{2565} a_{4}(n)-\frac{830816}{513} a_{4}\left(\frac{n}{2}\right) \\
& -\frac{2281984}{45} a_{4}\left(\frac{n}{4}\right)-40 a_{2}(n)+\frac{108216}{19} a_{2}\left(\frac{n}{3}\right)+56 a_{3}(n) \\
& -\frac{274428}{19} a_{3}\left(\frac{n}{3}\right)+\frac{1261440}{19} a_{5}(n)+\frac{10738944}{19} a_{5}\left(\frac{n}{2}\right)-280 a_{6}(n) \\
& +\frac{417344}{19} a_{6}\left(\frac{n}{2}\right)-\frac{520512}{19} a_{7}(n)+\frac{513408}{19} a_{8}(n)+\frac{673152}{19} a_{9}(n), \\
& N\left(1^{8}, 2^{8} ; n\right)=\frac{1}{4080} \sigma_{7}(n)-\frac{1}{4080} \sigma_{7}\left(\frac{n}{2}\right)-\frac{1}{255} \sigma_{7}\left(\frac{n}{4}\right)+\frac{256}{255} \sigma_{7}\left(\frac{n}{8}\right) \\
& -\frac{2}{17} a_{1}(n)-16 a_{1}\left(\frac{n}{2}\right)-\frac{512}{17} a_{1}\left(\frac{n}{4}\right)+16 a_{3}(n), \\
& N\left(1^{9}, 2,3,6^{5} ; n\right)=\frac{121}{6691200} \sigma_{7}(n)-\frac{121}{6691200} \sigma_{7}\left(\frac{n}{2}\right)+\frac{1053}{2230400} \sigma_{7}\left(\frac{n}{3}\right) \\
& -\frac{121}{836400} \sigma_{7}\left(\frac{n}{4}\right)-\frac{1053}{2230400} \sigma_{7}\left(\frac{n}{6}\right)+\frac{1936}{52275} \sigma_{7}\left(\frac{n}{8}\right)-\frac{1053}{278800} \sigma_{7}\left(\frac{n}{12}\right) \\
& +\frac{16848}{17425} \sigma_{7}\left(\frac{n}{24}\right)-\frac{26532499}{1308150} a_{1}(n)-\frac{123434}{285} a_{1}\left(\frac{n}{2}\right)-\frac{1773972}{8075} a_{1}\left(\frac{n}{3}\right) \\
& +\frac{1744119776}{654075} a_{1}\left(\frac{n}{4}\right)-\frac{453078}{95} a_{1}\left(\frac{n}{6}\right)+\frac{725237856}{8075} a_{1}\left(\frac{n}{12}\right) \\
& +\frac{1899551}{701100} \Delta_{3,8}(n)-\frac{90439717}{350550} \Delta_{3,8}\left(\frac{n}{2}\right)-\frac{3931178852}{175275} \Delta_{3,8}\left(\frac{n}{4}\right) \\
& -\frac{8399048512}{175275} \Delta_{3,8}\left(\frac{n}{8}\right)+\frac{135158}{7695} a_{4}(n)+\frac{22448}{57} a_{4}\left(\frac{n}{2}\right)+\frac{8483584}{405} a_{4}\left(\frac{n}{4}\right) \\
& -14 a_{2}(n)-\frac{24408}{19} a_{2}\left(\frac{n}{3}\right)+32 a_{3}(n)+\frac{33642}{19} a_{3}\left(\frac{n}{3}\right)+\frac{15264}{19} a_{5}(n) \\
& -\frac{4210944}{19} a_{5}\left(\frac{n}{2}\right)-108 a_{6}(n)-\frac{144928}{19} a_{6}\left(\frac{n}{2}\right) \\
& -\frac{77920}{19} a_{7}(n)-\frac{110272}{19} a_{8}(n)+\frac{270400}{19} a_{9}(n) \text {, }
\end{aligned}
$$




$$
\begin{aligned}
& N\left(1^{9}, 2,3^{3}, 6^{3} ; n\right)=\frac{121}{3345600} \sigma_{7}(n)-\frac{121}{3345600} \sigma_{7}\left(\frac{n}{2}\right)+\frac{1053}{1115200} \sigma_{7}\left(\frac{n}{3}\right)-\frac{121}{836400} \sigma_{7}\left(\frac{n}{4}\right) \\
& -\frac{1053}{1115200} \sigma_{7}\left(\frac{n}{6}\right)+\frac{1936}{52275} \sigma_{7}\left(\frac{n}{8}\right)-\frac{1053}{278800} \sigma_{7}\left(\frac{n}{12}\right)+\frac{16848}{17425} \sigma_{7}\left(\frac{n}{24}\right)-\frac{3563204}{654075} a_{1}(n) \\
& -\frac{1143488}{2565} a_{1}\left(\frac{n}{2}\right)-\frac{4953249}{8075} a_{1}\left(\frac{n}{3}\right)+\frac{1976614496}{654075} a_{1}\left(\frac{n}{4}\right)-\frac{1144764}{95} a_{1}\left(\frac{n}{6}\right)+\frac{636962976}{8075} a_{1}\left(\frac{n}{12}\right) \\
& +\frac{2652721}{350550} \Delta_{3,8}(n)-\frac{29311997}{175275} \Delta_{3,8}\left(\frac{n}{2}\right)-\frac{6529804772}{175275} \Delta_{3,8}\left(\frac{n}{4}\right)-\frac{9835452352}{175275} \Delta_{3,8}\left(\frac{n}{8}\right) \\
& -\frac{16444}{7695} a_{4}(n)+\frac{300208}{513} a_{4}\left(\frac{n}{2}\right)+\frac{12546304}{405} a_{4}\left(\frac{n}{4}\right)-10 a_{2}(n)-\frac{37206}{19} a_{2}\left(\frac{n}{3}\right)+28 a_{3}(n) \\
& +\frac{64908}{19} a_{3}\left(\frac{n}{3}\right)-\frac{121680}{19} a_{5}(n)-\frac{6271296}{19} a_{5}\left(\frac{n}{2}\right)-72 a_{6}(n)-\frac{221792}{19} a_{6}\left(\frac{n}{2}\right) \\
& -\frac{37120}{19} a_{7}(n)-\frac{196864}{19} a_{8}(n)+\frac{257536}{19} a_{9}(n) \text {, } \\
& N\left(1^{9}, 2,3^{5}, 6 ; n\right)=\frac{121}{1672800} \sigma_{7}(n)-\frac{121}{1672800} \sigma_{7}\left(\frac{n}{2}\right)+\frac{1053}{557600} \sigma_{7}\left(\frac{n}{3}\right)-\frac{121}{836400} \sigma_{7}\left(\frac{n}{4}\right) \\
& -\frac{1053}{557600} \sigma_{7}\left(\frac{n}{6}\right)+\frac{1936}{52275} \sigma_{7}\left(\frac{n}{8}\right)-\frac{1053}{278800} \sigma_{7}\left(\frac{n}{12}\right)+\frac{16848}{17425} \sigma_{7}\left(\frac{n}{24}\right)+\frac{2648399}{218025} a_{1}(n) \\
& -\frac{1299868}{2565} a_{1}\left(\frac{n}{2}\right)-\frac{8455293}{8075} a_{1}\left(\frac{n}{3}\right)+\frac{266917984}{72675} a_{1}\left(\frac{n}{4}\right)-\frac{1869264}{95} a_{1}\left(\frac{n}{6}\right)+\frac{376789536}{8075} a_{1}\left(\frac{n}{12}\right) \\
& +\frac{754612}{58425} \Delta_{3,8}(n)+\frac{3840422}{58425} \Delta_{3,8}\left(\frac{n}{2}\right)-\frac{3393395764}{58425} \Delta_{3,8}\left(\frac{n}{4}\right)-\frac{4110799424}{58425} \Delta_{3,8}\left(\frac{n}{8}\right) \\
& -\frac{64376}{2565} a_{4}(n)+\frac{367952}{513} a_{4}\left(\frac{n}{2}\right)+\frac{2084096}{45} a_{4}\left(\frac{n}{4}\right)-2 a_{2}(n)-\frac{45846}{19} a_{2}\left(\frac{n}{3}\right)+20 a_{3}(n) \\
& +\frac{92016}{19} a_{3}\left(\frac{n}{3}\right)-\frac{256176}{19} a_{5}(n)-\frac{9369792}{19} a_{5}\left(\frac{n}{2}\right)-\frac{335648}{19} a_{6}\left(\frac{n}{2}\right)-\frac{10176}{19} a_{7}(n) \\
& -\frac{331392}{19} a_{8}(n)+\frac{290688}{19} a_{9}(n) \text {, } \\
& N\left(1^{9}, 2^{3}, 3,6^{3} ; n\right)=\frac{73}{1338240} \sigma_{7}(n)-\frac{73}{1338240} \sigma_{7}\left(\frac{n}{2}\right)-\frac{243}{446080} \sigma_{7}\left(\frac{n}{3}\right)+\frac{73}{167280} \sigma_{7}\left(\frac{n}{4}\right) \\
& +\frac{243}{446080} \sigma_{7}\left(\frac{n}{6}\right)-\frac{1168}{10455} \sigma_{7}\left(\frac{n}{8}\right)-\frac{243}{55760} \sigma_{7}\left(\frac{n}{12}\right)+\frac{3888}{3485} \sigma_{7}\left(\frac{n}{24}\right)-\frac{14377981}{261630} a_{1}(n) \\
& -\frac{1022602}{2565} a_{1}\left(\frac{n}{2}\right)+\frac{1029087}{1615} a_{1}\left(\frac{n}{3}\right)+\frac{272238368}{130815} a_{1}\left(\frac{n}{4}\right)+\frac{990594}{95} a_{1}\left(\frac{n}{6}\right)+\frac{246390048}{1615} a_{1}\left(\frac{n}{12}\right) \\
& -\frac{1102621}{140220} \Delta_{3,8}(n)-\frac{7330709}{14022} \Delta_{3,8}\left(\frac{n}{2}\right)+\frac{402033028}{35055} \Delta_{3,8}\left(\frac{n}{4}\right)-\frac{1146114496}{35055} \Delta_{3,8}\left(\frac{n}{8}\right) \\
& +\frac{96638}{1539} a_{4}(n)+\frac{12176}{513} a_{4}\left(\frac{n}{2}\right)-\frac{177920}{81} a_{4}\left(\frac{n}{4}\right)-30 a_{2}(n)-\frac{6696}{19} a_{2}\left(\frac{n}{3}\right)+48 a_{3}(n) \\
& -\frac{21006}{19} a_{3}\left(\frac{n}{3}\right)+\frac{314784}{19} a_{5}(n)+\frac{502656}{19} a_{5}\left(\frac{n}{2}\right)-204 a_{6}(n)+\frac{31264}{19} a_{6}\left(\frac{n}{2}\right) \\
& -\frac{159968}{19} a_{7}(n)+\frac{106048}{19} a_{8}(n)+\frac{299840}{19} a_{9}(n) \text {, }
\end{aligned}
$$




$$
\begin{aligned}
& N\left(1^{9}, 2^{3}, 3^{3}, 6 ; n\right)=\frac{73}{669120} \sigma_{7}(n)-\frac{73}{669120} \sigma_{7}\left(\frac{n}{2}\right)-\frac{243}{223040} \sigma_{7}\left(\frac{n}{3}\right)+\frac{73}{167280} \sigma_{7}\left(\frac{n}{4}\right) \\
& +\frac{243}{223040} \sigma_{7}\left(\frac{n}{6}\right)-\frac{1168}{10455} \sigma_{7}\left(\frac{n}{8}\right)-\frac{243}{55760} \sigma_{7}\left(\frac{n}{12}\right)+\frac{3888}{3485} \sigma_{7}\left(\frac{n}{24}\right)-\frac{12354896}{130815} a_{1}(n) \\
& -\frac{797176}{2565} a_{1}\left(\frac{n}{2}\right)+\frac{2970819}{1615} a_{1}\left(\frac{n}{3}\right)+\frac{132741536}{130815} a_{1}\left(\frac{n}{4}\right)+\frac{3065652}{95} a_{1}\left(\frac{n}{6}\right)+\frac{299354976}{1615} a_{1}\left(\frac{n}{12}\right) \\
& -\frac{1591751}{70110} \Delta_{3,8}(n)-\frac{22717481}{35055} \Delta_{3,8}\left(\frac{n}{2}\right)+\frac{392241716}{7011} \Delta_{3,8}\left(\frac{n}{4}\right)-\frac{284272192}{35055} \Delta_{3,8}\left(\frac{n}{8}\right) \\
& +\frac{180212}{1539} a_{4}(n)-\frac{340720}{513} a_{4}\left(\frac{n}{2}\right)-\frac{2615552}{81} a_{4}\left(\frac{n}{4}\right)-42 a_{2}(n)+\frac{31698}{19} a_{2}\left(\frac{n}{3}\right)+60 a_{3}(n) \\
& -\frac{114804}{19} a_{3}\left(\frac{n}{3}\right)+\frac{725616}{19} a_{5}(n)+\frac{6683712}{19} a_{5}\left(\frac{n}{2}\right)-264 a_{6}(n)+\frac{261856}{19} a_{6}\left(\frac{n}{2}\right) \\
& -\frac{282368}{19} a_{7}(n)+\frac{365824}{19} a_{8}(n)+\frac{338432}{19} a_{9}(n) \text {, } \\
& N\left(1^{9}, 2^{5}, 3,6 ; n\right)=\frac{1093}{6691200} \sigma_{7}(n)-\frac{1093}{6691200} \sigma_{7}\left(\frac{n}{2}\right)+\frac{729}{2230400} \sigma_{7}\left(\frac{n}{3}\right)-\frac{1093}{836400} \sigma_{7}\left(\frac{n}{4}\right) \\
& -\frac{729}{2230400} \sigma_{7}\left(\frac{n}{6}\right)+\frac{17488}{52275} \sigma_{7}\left(\frac{n}{8}\right)-\frac{729}{278800} \sigma_{7}\left(\frac{n}{12}\right)+\frac{11664}{17425} \sigma_{7}\left(\frac{n}{24}\right)+\frac{17032631}{436050} a_{1}(n) \\
& -\frac{779554}{2565} a_{1}\left(\frac{n}{2}\right)-\frac{14247846}{8075} a_{1}\left(\frac{n}{3}\right)+\frac{579121696}{218025} a_{1}\left(\frac{n}{4}\right)-\frac{3061422}{95} a_{1}\left(\frac{n}{6}\right)+\frac{399407328}{8075} a_{1}\left(\frac{n}{12}\right) \\
& +\frac{5090281}{233700} \Delta_{3,8}(n)-\frac{19936878}{116850} \Delta_{3,8}\left(\frac{n}{2}\right)-\frac{3842024572}{58425} \Delta_{3,8}\left(\frac{n}{4}\right)-\frac{3164610752}{58425} \Delta_{3,8}\left(\frac{n}{8}\right) \\
& -\frac{156262}{2565} a_{4}(n)+\frac{645104}{513} a_{4}\left(\frac{n}{2}\right)+\frac{6212864}{135} a_{4}\left(\frac{n}{4}\right)+2 a_{2}(n)-\frac{76464}{19} a_{2}\left(\frac{n}{3}\right)+16 a_{3}(n) \\
& +\frac{159570}{19} a_{3}\left(\frac{n}{3}\right)-\frac{560736}{19} a_{5}(n)-\frac{9485568}{19} a_{5}\left(\frac{n}{2}\right)-28 a_{6}(n)-\frac{349344}{19} a_{6}\left(\frac{n}{2}\right) \\
& +\frac{141600}{19} a_{7}(n)-\frac{351168}{19} a_{8}(n)+\frac{27456}{19} a_{9}(n) \text {, } \\
& N\left(1^{10}, 6^{6} ; n\right)=\frac{61}{3345600} \sigma_{7}(n)-\frac{61}{3345600} \sigma_{7}\left(\frac{n}{2}\right)-\frac{567}{1115200} \sigma_{7}\left(\frac{n}{3}\right)+\frac{61}{418200} \sigma_{7}\left(\frac{n}{4}\right) \\
& +\frac{567}{1115200} \sigma_{7}\left(\frac{n}{6}\right)-\frac{1952}{52275} \sigma_{7}\left(\frac{n}{8}\right)-\frac{567}{139400} \sigma_{7}\left(\frac{n}{12}\right)+\frac{18144}{17425} \sigma_{7}\left(\frac{n}{24}\right)-\frac{23816204}{654075} a_{1}(n) \\
& -\frac{1050332}{2565} a_{1}\left(\frac{n}{2}\right)+\frac{171351}{8075} a_{1}\left(\frac{n}{3}\right)+\frac{1396451648}{654075} a_{1}\left(\frac{n}{4}\right)-\frac{27396}{95} a_{1}\left(\frac{n}{6}\right)+\frac{1048557888}{8075} a_{1}\left(\frac{n}{12}\right) \\
& -\frac{90779}{350550} \Delta_{3,8}(n)-\frac{98876807}{175275} \Delta_{3,8}\left(\frac{n}{2}\right)-\frac{1125294056}{175275} \Delta_{3,8}\left(\frac{n}{4}\right)-\frac{6365435776}{175275} \Delta_{3,8}\left(\frac{n}{8}\right) \\
& +\frac{282116}{7695} a_{4}(n)+\frac{213472}{513} a_{4}\left(\frac{n}{2}\right)+\frac{3523072}{405} a_{4}\left(\frac{n}{4}\right)-20 a_{2}(n)-\frac{24732}{19} a_{2}\left(\frac{n}{3}\right)+40 a_{3}(n) \\
& +\frac{24732}{19} a_{3}\left(\frac{n}{3}\right)+\frac{99072}{19} a_{5}(n)-\frac{1768704}{19} a_{5}\left(\frac{n}{2}\right)-200 a_{6}(n)-\frac{56128}{19} a_{6}\left(\frac{n}{2}\right) \\
& -\frac{84160}{19} a_{7}(n)-\frac{1408}{19} a_{8}(n)+\frac{209536}{19} a_{9}(n) \text {, }
\end{aligned}
$$




$$
\begin{aligned}
& N\left(1^{10}, 3^{2}, 6^{4} ; n\right)=\frac{61}{1672800} \sigma_{7}(n)-\frac{61}{1672800} \sigma_{7}\left(\frac{n}{2}\right)-\frac{567}{557600} \sigma_{7}\left(\frac{n}{3}\right) \\
& +\frac{61}{418200} \sigma_{7}\left(\frac{n}{4}\right)+\frac{567}{557600} \sigma_{7}\left(\frac{n}{6}\right)-\frac{1952}{52275} \sigma_{7}\left(\frac{n}{8}\right)-\frac{567}{139400} \sigma_{7}\left(\frac{n}{12}\right) \\
& +\frac{18144}{17425} \sigma_{7}\left(\frac{n}{24}\right)-\frac{32787328}{654075} a_{1}(n)-\frac{1351304}{2565} a_{1}\left(\frac{n}{2}\right)+\frac{2784582}{8075} a_{1}\left(\frac{n}{3}\right) \\
& +\frac{1708859968}{654075} a_{1}\left(\frac{n}{4}\right)+\frac{417168}{95} a_{1}\left(\frac{n}{6}\right)+\frac{908630208}{8075} a_{1}\left(\frac{n}{12}\right) \\
& -\frac{745139}{175275} \Delta_{3,8}(n)-\frac{111705274}{175275} \Delta_{3,8}\left(\frac{n}{2}\right)-\frac{876486376}{175275} \Delta_{3,8}\left(\frac{n}{4}\right) \\
& -\frac{7764185216}{175275} \Delta_{3,8}\left(\frac{n}{8}\right)+\frac{418312}{7695} a_{4}(n)+\frac{175840}{513} a_{4}\left(\frac{n}{2}\right) \\
& +\frac{4070912}{405} a_{4}\left(\frac{n}{4}\right)-20 a_{2}(n)-\frac{6372}{19} a_{2}\left(\frac{n}{3}\right)+40 a_{3}(n)-\frac{12096}{19} a_{3}\left(\frac{n}{3}\right) \\
& +\frac{198144}{19} a_{5}(n)-\frac{1939584}{19} a_{5}\left(\frac{n}{2}\right)-240 a_{6}(n)-\frac{57536}{19} a_{6}\left(\frac{n}{2}\right) \\
& -\frac{153728}{19} a_{7}(n)-\frac{2816}{19} a_{8}(n)+\frac{360704}{19} a_{9}(n), \\
& N\left(1^{10}, 3^{4}, 6^{2} ; n\right)=\frac{61}{836400} \sigma_{7}(n)-\frac{61}{836400} \sigma_{7}\left(\frac{n}{2}\right)-\frac{567}{278800} \sigma_{7}\left(\frac{n}{3}\right) \\
& +\frac{61}{418200} \sigma_{7}\left(\frac{n}{4}\right)+\frac{567}{278800} \sigma_{7}\left(\frac{n}{6}\right)-\frac{1952}{52275} \sigma_{7}\left(\frac{n}{8}\right)-\frac{567}{139400} \sigma_{7}\left(\frac{n}{12}\right) \\
& +\frac{18144}{17425} \sigma_{7}\left(\frac{n}{24}\right)-\frac{37040326}{654075} a_{1}(n)-\frac{156664}{2565} a_{1}\left(\frac{n}{2}\right)-\frac{1666206}{8075} a_{1}\left(\frac{n}{3}\right) \\
& +\frac{1708859968}{654075} a_{1}\left(\frac{n}{4}\right)-\frac{50688}{95} a_{1}\left(\frac{n}{6}\right)+\frac{908630208}{8075} a_{1}\left(\frac{n}{12}\right)-\frac{1654688}{175275} \Delta_{3,8}(n) \\
& -\frac{155476828}{175275} \Delta_{3,8}\left(\frac{n}{2}\right)-\frac{1042506856}{175275} \Delta_{3,8}\left(\frac{n}{4}\right)-\frac{7764185216}{175275} \Delta_{3,8}\left(\frac{n}{8}\right) \\
& +\frac{508144}{7695} a_{4}(n)+\frac{27104}{57} a_{4}\left(\frac{n}{2}\right)+\frac{4070912}{405} a_{4}\left(\frac{n}{4}\right)-20 a_{2}(n)-\frac{6372}{19} a_{2}\left(\frac{n}{3}\right) \\
& +40 a_{3}(n)-\frac{12096}{19} a_{3}\left(\frac{n}{3}\right)+\frac{181728}{19} a_{5}(n)-\frac{1939584}{19} a_{5}\left(\frac{n}{2}\right)-320 a_{6}(n) \\
& -\frac{57536}{19} a_{6}\left(\frac{n}{2}\right)-\frac{153728}{19} a_{7}(n)-\frac{2816}{19} a_{8}(n)+\frac{360704}{19} a_{9}(n), \\
& N\left(1^{10}, 3^{6} ; n\right)=\frac{61}{418200} \sigma_{7}(n)-\frac{567}{139400} \sigma_{7}\left(\frac{n}{3}\right)-\frac{1952}{52275} \sigma_{7}\left(\frac{n}{4}\right) \\
& +\frac{18144}{17425} \sigma_{7}\left(\frac{n}{12}\right)-\frac{509936}{11475} a_{1}(n)-\frac{2039936}{11475} a_{1}\left(\frac{n}{2}\right)-\frac{826848}{425} a_{1}\left(\frac{n}{3}\right) \\
& -\frac{6334848}{425} a_{1}\left(\frac{n}{6}\right)-\frac{12256}{1025} \Delta_{3,8}(n)-\frac{50832}{25} \Delta_{3,8}\left(\frac{n}{2}\right)-\frac{2656256}{1025} \Delta_{3,8}\left(\frac{n}{4}\right) \\
& +\frac{10304}{135} a_{4}(n)+\frac{180224}{135} a_{4}\left(\frac{n}{2}\right)-6912 a_{5}(n)-640 a_{6}(n) \text {, }
\end{aligned}
$$




$$
\begin{aligned}
& N\left(1^{10}, 2^{2}, 6^{4} ; n\right)=\frac{91}{1672800} \sigma_{7}(n)-\frac{91}{1672800} \sigma_{7}\left(\frac{n}{2}\right)+\frac{243}{557600} \sigma_{7}\left(\frac{n}{3}\right)-\frac{91}{209100} \sigma_{7}\left(\frac{n}{4}\right) \\
& -\frac{243}{557600} \sigma_{7}\left(\frac{n}{6}\right)+\frac{5824}{52275} \sigma_{7}\left(\frac{n}{8}\right)-\frac{243}{69700} \sigma_{7}\left(\frac{n}{12}\right)+\frac{15552}{17425} \sigma_{7}\left(\frac{n}{24}\right)-\frac{19823588}{654075} a_{1}(n) \\
& -\frac{10584}{19} a_{1}\left(\frac{n}{2}\right)-\frac{48078}{8075} a_{1}\left(\frac{n}{3}\right)+\frac{2017890944}{654075} a_{1}\left(\frac{n}{4}\right)-\frac{39240}{19} a_{1}\left(\frac{n}{6}\right)+\frac{605787264}{8075} a_{1}\left(\frac{n}{12}\right) \\
& +\frac{12431}{175275} \Delta_{3,8}(n)-\frac{35643194}{175275} \Delta_{3,8}\left(\frac{n}{2}\right)-\frac{4111033328}{175275} \Delta_{3,8}\left(\frac{n}{4}\right)-\frac{9710326528}{175275} \Delta_{3,8}\left(\frac{n}{8}\right) \\
& +\frac{232472}{7695} a_{4}(n)+\frac{16192}{57} a_{4}\left(\frac{n}{2}\right)+\frac{9696256}{405} a_{4}\left(\frac{n}{4}\right)-12 a_{2}(n)-\frac{14040}{19} a_{2}\left(\frac{n}{3}\right)+32 a_{3}(n) \\
& +\frac{14040}{19} a_{3}\left(\frac{n}{3}\right)+\frac{107712}{19} a_{5}(n)-\frac{4747776}{19} a_{5}\left(\frac{n}{2}\right)-112 a_{6}(n)-\frac{161152}{19} a_{6}\left(\frac{n}{2}\right) \\
& -\frac{113536}{19} a_{7}(n)-\frac{124672}{19} a_{8}(n)+\frac{390400}{19} a_{9}(n) \text {, } \\
& N\left(1^{10}, 2^{2}, 3^{2}, 6^{2} ; n\right)=\frac{91}{836400} \sigma_{7}(n)-\frac{91}{836400} \sigma_{7}\left(\frac{n}{2}\right)+\frac{243}{278800} \sigma_{7}\left(\frac{n}{3}\right)-\frac{91}{209100} \sigma_{7}\left(\frac{n}{4}\right) \\
& -\frac{243}{278800} \sigma_{7}\left(\frac{n}{6}\right)+\frac{5824}{52275} \sigma_{7}\left(\frac{n}{8}\right)-\frac{243}{69700} \sigma_{7}\left(\frac{n}{12}\right)+\frac{15552}{17425} \sigma_{7}\left(\frac{n}{24}\right)+\frac{2429998}{218025} a_{1}(n) \\
& -\frac{45256}{95} a_{1}\left(\frac{n}{2}\right)-\frac{9415386}{8075} a_{1}\left(\frac{n}{3}\right)+\frac{723490688}{218025} a_{1}\left(\frac{n}{4}\right)-\frac{2147256}{95} a_{1}\left(\frac{n}{6}\right)+\frac{569165184}{8075} a_{1}\left(\frac{n}{12}\right) \\
& +\frac{840724}{58425} \Delta_{3,8}(n)-\frac{10538536}{58425} \Delta_{3,8}\left(\frac{n}{2}\right)-\frac{3238046416}{58425} \Delta_{3,8}\left(\frac{n}{4}\right)-\frac{3728128256}{58425} \Delta_{3,8}\left(\frac{n}{8}\right) \\
& -\frac{508144}{2565} a_{4}(n)+\frac{54080}{57} a_{4}\left(\frac{n}{2}\right)+\frac{5757952}{405} a_{4}\left(\frac{n}{4}\right)-4 a_{2}(n)-\frac{57996}{19} a_{2}\left(\frac{n}{3}\right)+24 a_{3}(n) \\
& +\frac{113400}{19} a_{3}\left(\frac{n}{3}\right)-\frac{314496}{19} a_{5}(n)-\frac{8697600}{19} a_{5}\left(\frac{n}{2}\right)-64 a_{6}(n)-\frac{313472}{19} a_{6}\left(\frac{n}{2}\right) \\
& +\frac{37632}{19} a_{7}(n)-\frac{296448}{19} a_{8}(n)+\frac{360704}{19} a_{9}(n), \\
& N\left(1^{10}, 2^{2}, 3^{4} ; n\right)=\frac{91}{418200} \sigma_{7}(n)-\frac{91}{418200} \sigma_{7}\left(\frac{n}{2}\right)+\frac{243}{139400} \sigma_{7}\left(\frac{n}{3}\right)-\frac{91}{209100} \sigma_{7}\left(\frac{n}{4}\right) \\
& -\frac{243}{139400} \sigma_{7}\left(\frac{n}{6}\right)+\frac{5824}{52275} \sigma_{7}\left(\frac{n}{8}\right)-\frac{243}{69700} \sigma_{7}\left(\frac{n}{12}\right)+\frac{15552}{17425} \sigma_{7}\left(\frac{n}{24}\right)+\frac{36848968}{654075} a_{1}(n) \\
& -\frac{538048}{855} a_{1}\left(\frac{n}{2}\right)-\frac{20489292}{8075} a_{1}\left(\frac{n}{3}\right)+\frac{3095663744}{654075} a_{1}\left(\frac{n}{4}\right)-\frac{4377312}{95} a_{1}\left(\frac{n}{6}\right)-\frac{155051136}{8075} a_{1}\left(\frac{n}{12}\right) \\
& +\frac{5488784}{175275} \Delta_{3,8}(n)-\frac{1614776}{175275} \Delta_{3,8}\left(\frac{n}{2}\right)-\frac{19605038288}{175275} \Delta_{3,8}\left(\frac{n}{4}\right)-\frac{16900611328}{175275} \Delta_{3,8}\left(\frac{n}{8}\right) \\
& -\frac{644512}{7695} a_{4}(n)+\frac{293696}{171} a_{4}\left(\frac{n}{2}\right)+\frac{33442816}{405} a_{4}\left(\frac{n}{4}\right)+16 a_{2}(n)-\frac{85320}{19} a_{2}\left(\frac{n}{3}\right) \\
& +\frac{196128}{19} a_{3}\left(\frac{n}{3}\right)-\frac{759744}{19} a_{5}(n)-\frac{16800000}{19} a_{5}\left(\frac{n}{2}\right)-\frac{613760}{19} a_{6}\left(\frac{n}{2}\right) \\
& +\frac{1333376}{19} a_{7}(n)-\frac{659968}{19} a_{8}(n)+\frac{220672}{19} a_{9}(n) \text {, }
\end{aligned}
$$




$$
\begin{aligned}
& N\left(1^{10}, 2^{4}, 6^{2} ; n\right)=\frac{547}{3345600} \sigma_{7}(n)-\frac{547}{3345600} \sigma_{7}\left(\frac{n}{2}\right)-\frac{729}{1115200} \sigma_{7}\left(\frac{n}{3}\right) \\
& +\frac{547}{418200} \sigma_{7}\left(\frac{n}{4}\right)+\frac{729}{1115200} \sigma_{7}\left(\frac{n}{6}\right)-\frac{17504}{52275} \sigma_{7}\left(\frac{n}{8}\right)-\frac{729}{139400} \sigma_{7}\left(\frac{n}{12}\right) \\
& +\frac{23328}{17425} \sigma_{7}\left(\frac{n}{24}\right)-\frac{3373492}{723675} a_{1}(n)-\frac{334684}{855} a_{1}\left(\frac{n}{2}\right)+\frac{1293057}{8075} a_{1}\left(\frac{n}{3}\right) \\
& +\frac{173397184}{72675} a_{1}\left(\frac{n}{4}\right)+\frac{93204}{95} a_{1}\left(\frac{n}{6}\right)+\frac{1708883136}{8075} a_{1}\left(\frac{n}{12}\right) \\
& -\frac{230551}{116850} \Delta_{3,8}(n)-\frac{42961123}{58425} \Delta_{3,8}\left(\frac{n}{2}\right)+\frac{120936416}{58425} \Delta_{3,8}\left(\frac{n}{4}\right) \\
& -\frac{2258033024}{58425} \Delta_{3,8}\left(\frac{n}{8}\right)+\frac{41308}{855} a_{4}(n)+\frac{75488}{171} a_{4}\left(\frac{n}{2}\right) \\
& +\frac{114176}{45} a_{4}\left(\frac{n}{4}\right)-36 a_{2}(n)-\frac{40500}{19} a_{2}\left(\frac{n}{3}\right)+56 a_{3}(n) \\
& +\frac{40500}{19} a_{3}\left(\frac{n}{3}\right)+\frac{150336}{19} a_{5}(n)-\frac{582912}{19} a_{5}\left(\frac{n}{2}\right)-248 a_{6}(n) \\
& -\frac{11200}{19} a_{6}\left(\frac{n}{2}\right)-\frac{85056}{19} a_{7}(n)+\frac{84864}{19} a_{8}(n)+\frac{216960}{19} a_{9}(n), \\
& N\left(1^{10}, 2^{4}, 3^{2} ; n\right)=\frac{547}{1672800} \sigma_{7}(n)-\frac{547}{1672800} \sigma_{7}\left(\frac{n}{2}\right)-\frac{729}{557600} \sigma_{7}\left(\frac{n}{3}\right) \\
& +\frac{547}{418200} \sigma_{7}\left(\frac{n}{4}\right)+\frac{729}{557600} \sigma_{7}\left(\frac{n}{6}\right)-\frac{17504}{52275} \sigma_{7}\left(\frac{n}{8}\right)-\frac{729}{139400} \sigma_{7}\left(\frac{n}{12}\right) \\
& +\frac{23328}{17425} \sigma_{7}\left(\frac{n}{24}\right)-\frac{34392092}{218025} a_{1}(n)-\frac{256840}{513} a_{1}\left(\frac{n}{2}\right)+\frac{28391094}{8075} a_{1}\left(\frac{n}{3}\right) \\
& +\frac{87292864}{72675} a_{1}\left(\frac{n}{4}\right)+\frac{1188000}{95} a_{1}\left(\frac{n}{6}\right)+\frac{1587560256}{8075} a_{1}\left(\frac{n}{12}\right) \\
& -\frac{2535571}{58425} \Delta_{3,8}(n)-\frac{38974346}{58425} \Delta_{3,8}\left(\frac{n}{2}\right)+\frac{5731226296}{58425} \Delta_{3,8}\left(\frac{n}{4}\right) \\
& -\frac{307928704}{58425} \Delta_{3,8}\left(\frac{n}{8}\right)+\frac{515528}{2565} a_{4}(n)-\frac{835040}{513} a_{4}\left(\frac{n}{2}\right) \\
& -\frac{2481664}{45} a_{4}\left(\frac{n}{4}\right)-52 a_{2}(n)+\frac{96876}{19} a_{2}\left(\frac{n}{3}\right)+72 a_{3}(n) \\
& -\frac{263088}{19} a_{3}\left(\frac{n}{3}\right)+\frac{1354752}{19} a_{5}(n)+\frac{11576448}{19} a_{5}\left(\frac{n}{2}\right) \\
& -336 a_{6}(n)+\frac{454336}{19} a_{6}\left(\frac{n}{2}\right)-\frac{536448}{19} a_{7}(n) \\
& +\frac{584448}{19} a_{8}(n)+\frac{655104}{19} a_{9}(n) \text {, } \\
& N\left(1^{10}, 2^{6} ; n\right)=\frac{1}{2040} \sigma_{7}(n)-\frac{1}{2040} \sigma_{7}\left(\frac{n}{2}\right)-\frac{1}{255} \sigma_{7}\left(\frac{n}{4}\right)+\frac{256}{255} \sigma_{7}\left(\frac{n}{8}\right) \\
& -\frac{4}{17} a_{1}(n)-16 a_{1}\left(\frac{n}{2}\right)-\frac{512}{17} a_{1}\left(\frac{n}{4}\right)+4 a_{2}(n)+16 a_{3}(n),
\end{aligned}
$$




$$
\begin{aligned}
& N\left(1^{11}, 2,3,6^{3} ; n\right)=\frac{73}{669120} \sigma_{7}(n)-\frac{73}{669120} \sigma_{7}\left(\frac{n}{2}\right)-\frac{243}{223040} \sigma_{7}\left(\frac{n}{3}\right)+\frac{73}{167280} \sigma_{7}\left(\frac{n}{4}\right) \\
& +\frac{243}{223040} \sigma_{7}\left(\frac{n}{6}\right)-\frac{1168}{10455} \sigma_{7}\left(\frac{n}{8}\right)-\frac{243}{55760} \sigma_{7}\left(\frac{n}{12}\right)+\frac{3888}{3485} \sigma_{7}\left(\frac{n}{24}\right)-\frac{1733968}{26163} a_{1}(n) \\
& -\frac{1312544}{2565} a_{1}\left(\frac{n}{2}\right)+\frac{225495}{323} a_{1}\left(\frac{n}{3}\right)+\frac{60791584}{26163} a_{1}\left(\frac{n}{4}\right)+\frac{981468}{95} a_{1}\left(\frac{n}{6}\right)+\frac{53901792}{8075} a_{1}\left(\frac{n}{12}\right) \\
& -\frac{120763}{14022} \Delta_{3,8}(n)-\frac{23961257}{35055} \Delta_{3,8}\left(\frac{n}{2}\right)+\frac{407513908}{35055} \Delta_{3,8}\left(\frac{n}{4}\right)-\frac{257785664}{7011} \Delta_{3,8}\left(\frac{n}{8}\right) \\
& +\frac{115172}{1539} a_{4}(n)+\frac{65488}{513} a_{4}\left(\frac{n}{2}\right)-\frac{114944}{81} a_{4}\left(\frac{n}{4}\right)-30 a_{2}(n)-\frac{9450}{19} a_{2}\left(\frac{n}{3}\right)+52 a_{3}(n) \\
& -\frac{18252}{19} a_{3}\left(\frac{n}{3}\right)+\frac{354096}{19} a_{5}(n)+\frac{347712}{19} a_{5}\left(\frac{n}{2}\right)-264 a_{6}(n)+\frac{26976}{19} a_{6}\left(\frac{n}{2}\right) \\
& -\frac{161024}{19} a_{7}(n)+\frac{113920}{19} a_{8}(n)+\frac{346112}{19} a_{9}(n) \text {, } \\
& N\left(1^{11}, 2,3^{3}, 6 ; n\right)=\frac{73}{334560} \sigma_{7}(n)-\frac{73}{334560} \sigma_{7}\left(\frac{n}{2}\right)-\frac{243}{111520} \sigma_{7}\left(\frac{n}{3}\right)+\frac{73}{167280} \sigma_{7}\left(\frac{n}{4}\right) \\
& +\frac{243}{111520} \sigma_{7}\left(\frac{n}{6}\right)-\frac{1168}{10455} \sigma_{7}\left(\frac{n}{8}\right)-\frac{243}{55760} \sigma_{7}\left(\frac{n}{12}\right)+\frac{3888}{3485} \sigma_{7}\left(\frac{n}{24}\right)-\frac{13727401}{130815} a_{1}(n) \\
& -\frac{1239796}{2565} a_{1}\left(\frac{n}{2}\right)+\frac{2925549}{1615} a_{1}\left(\frac{n}{3}\right)+\frac{203091616}{130815} a_{1}\left(\frac{n}{4}\right)+\frac{2900592}{95} a_{1}\left(\frac{n}{6}\right)+\frac{288094176}{1615} a_{1}\left(\frac{n}{12}\right) \\
& -\frac{783518}{35055} \Delta_{3,8}(n)-\frac{31490206}{35055} \Delta_{3,8}\left(\frac{n}{2}\right)+\frac{344985772}{7011} \Delta_{3,8}\left(\frac{n}{4}\right)-\frac{639194432}{35055} \Delta_{3,8}\left(\frac{n}{8}\right) \\
& +\frac{195736}{1539} a_{4}(n)-\frac{210832}{513} a_{4}\left(\frac{n}{2}\right)-\frac{2123008}{81} a_{4}\left(\frac{n}{4}\right)-38 a_{2}(n)+\frac{33102}{19} a_{2}\left(\frac{n}{3}\right)+60 a_{3}(n) \\
& -\frac{116208}{19} a_{3}\left(\frac{n}{3}\right)+\frac{718128}{19} a_{5}(n)+\frac{5490624}{19} a_{5}\left(\frac{n}{2}\right)-352 a_{6}(n)+\frac{220576}{19} a_{6}\left(\frac{n}{2}\right) \\
& -\frac{297280}{19} a_{7}(n)+\frac{325760}{19} a_{8}(n)+\frac{430720}{19} a_{9}(n) \text {, } \\
& N\left(1^{11}, 2^{3}, 3,6 ; n\right)=\frac{1093}{3345600} \sigma_{7}(n)-\frac{1093}{3345600} \sigma_{7}\left(\frac{n}{2}\right)+\frac{729}{1115200} \sigma_{7}\left(\frac{n}{3}\right)+\frac{1093}{836400} \sigma_{7}\left(\frac{n}{4}\right) \\
& -\frac{729}{1115200} \sigma_{7}\left(\frac{n}{6}\right)+\frac{17488}{52275} \sigma_{7}\left(\frac{n}{8}\right)-\frac{729}{278800} \sigma_{7}\left(\frac{n}{12}\right)+\frac{11644}{17425} \sigma_{7}\left(\frac{n}{24}\right)+\frac{7141436}{218025} a_{1}(n) \\
& -\frac{941816}{2565} a_{1}\left(\frac{n}{2}\right)-\frac{13585077}{8075} a_{1}\left(\frac{n}{3}\right)+\frac{631987616}{218025} a_{1}\left(\frac{n}{4}\right)-\frac{3070548}{95} a_{1}\left(\frac{n}{6}\right)+\frac{515001888}{8075} a_{1}\left(\frac{n}{12}\right) \\
& +\frac{808837}{38950} \Delta_{3,8}(n)-\frac{3589729}{19475} \Delta_{3,8}\left(\frac{n}{2}\right)-\frac{1277629924}{19475} \Delta_{3,8}\left(\frac{n}{4}\right)-\frac{1134211264}{19475} \Delta_{3,8}\left(\frac{n}{8}\right) \\
& -\frac{137684}{2565} a_{4}(n)+\frac{640048}{513} a_{4}\left(\frac{n}{2}\right)+\frac{6317824}{135} a_{4}\left(\frac{n}{4}\right)+2 a_{2}(n)-\frac{79218}{19} a_{2}\left(\frac{n}{3}\right)+20 a_{3}(n) \\
& +\frac{162324}{19} a_{3}\left(\frac{n}{3}\right)-\frac{521424}{19} a_{5}(n)-\frac{9640512}{19} a_{5}\left(\frac{n}{2}\right)-40 a_{6}(n)-\frac{353632}{19} a_{6}\left(\frac{n}{2}\right) \\
& +\frac{140544}{19} a_{7}(n)-\frac{343296}{19} a_{8}(n)+\frac{73728}{19} a_{9}(n) \text {, }
\end{aligned}
$$




$$
\begin{aligned}
& N\left(1^{12}, 6^{4} ; n\right)=\frac{91}{836400} \sigma_{7}(n)-\frac{91}{836400} \sigma_{7}\left(\frac{n}{2}\right)+\frac{243}{278800} \sigma_{7}\left(\frac{n}{3}\right) \\
& -\frac{91}{209100} \sigma_{7}\left(\frac{n}{4}\right)-\frac{243}{278800} \sigma_{7}\left(\frac{n}{6}\right)+\frac{5824}{52275} \sigma_{7}\left(\frac{n}{8}\right)-\frac{243}{69700} \sigma_{7}\left(\frac{n}{12}\right) \\
& +\frac{15552}{17425} \sigma_{7}\left(\frac{n}{24}\right)-\frac{28703596}{654075} a_{1}(n)-\frac{1073488}{2565} a_{1}\left(\frac{n}{2}\right)+\frac{2483424}{8075} a_{1}\left(\frac{n}{3}\right) \\
& +\frac{1245280384}{654075} a_{1}\left(\frac{n}{4}\right)+\frac{427536}{95} a_{1}\left(\frac{n}{6}\right)+\frac{1293381504}{8075} a_{1}\left(\frac{n}{12}\right) \\
& -\frac{666398}{175275} \Delta_{3,8}(n)-\frac{24418468}{175275} \Delta_{3,8}\left(\frac{n}{2}\right)+\frac{724178672}{175275} \Delta_{3,8}\left(\frac{n}{4}\right) \\
& -\frac{5468158208}{175275} \Delta_{3,8}\left(\frac{n}{8}\right)+\frac{366544}{7695} a_{4}(n)-\frac{43840}{513} a_{4}\left(\frac{n}{2}\right) \\
& +\frac{1104896}{405} a_{4}\left(\frac{n}{4}\right)-24 a_{2}(n)-\frac{30672}{19} a_{2}\left(\frac{n}{3}\right)+48 a_{3}(n) \\
& +\frac{30672}{19} a_{3}\left(\frac{n}{3}\right)+\frac{294912}{19} a_{5}(n)-\frac{595200}{19} a_{5}\left(\frac{n}{2}\right)-96 a_{6}(n) \\
& -\frac{13184}{19} a_{6}\left(\frac{n}{2}\right)-\frac{58112}{19} a_{7}(n)+\frac{67072}{19} a_{8}(n)+\frac{206336}{19} a_{9}(n), \\
& N\left(1^{12}, 3^{2}, 6^{2} ; n\right)=\frac{91}{418200} \sigma_{7}(n)-\frac{91}{418200} \sigma_{7}\left(\frac{n}{2}\right)+\frac{243}{139400} \sigma_{7}\left(\frac{n}{3}\right) \\
& -\frac{91}{209100} \sigma_{7}\left(\frac{n}{4}\right)-\frac{243}{139400} \sigma_{7}\left(\frac{n}{6}\right)+\frac{5824}{52275} \sigma_{7}\left(\frac{n}{8}\right)-\frac{243}{69700} \sigma_{7}\left(\frac{n}{12}\right) \\
& +\frac{15552}{17425} \sigma_{7}\left(\frac{n}{24}\right)-\frac{16455892}{654075} a_{1}(n)-\frac{856432}{2565} a_{1}\left(\frac{n}{2}\right)+\frac{14820048}{8075} a_{1}\left(\frac{n}{3}\right) \\
& +\frac{1245280384}{654075} a_{1}\left(\frac{n}{4}\right)+\frac{1461744}{95} a_{1}\left(\frac{n}{6}\right)+\frac{1293381504}{8075} a_{1}\left(\frac{n}{12}\right) \\
& +\frac{2336704}{175275} \Delta_{3,8}(n)+\frac{10816424}{175275} \Delta_{3,8}\left(\frac{n}{2}\right)+\frac{751100912}{175275} \Delta_{3,8}\left(\frac{n}{4}\right) \\
& -\frac{5468158208}{175275} \Delta_{3,8}\left(\frac{n}{8}\right)+\frac{90208}{7695} a_{4}(n)+\frac{14528}{513} a_{4}\left(\frac{n}{2}\right)+\frac{1104896}{405} a_{4}\left(\frac{n}{4}\right) \\
& -24 a_{2}(n)-\frac{30672}{19} a_{2}\left(\frac{n}{3}\right)+48 a_{3}(n)+\frac{30672}{19} a_{3}\left(\frac{n}{3}\right)+\frac{196416}{19} a_{5}(n) \\
& -\frac{595200}{19} a_{5}\left(\frac{n}{2}\right)-\frac{13184}{19} a_{6}\left(\frac{n}{2}\right)-\frac{58112}{19} a_{7}(n)+\frac{67072}{19} a_{8}(n)+\frac{206336}{19} a_{9}(n) \text {, } \\
& N\left(1^{12}, 3^{4} ; n\right)=\frac{91}{209100} \sigma_{7}(n)-\frac{91}{104550} \sigma_{7}\left(\frac{n}{2}\right)+\frac{243}{69700} \sigma_{7}\left(\frac{n}{3}\right)+\frac{5824}{52275} \sigma_{7}\left(\frac{n}{4}\right)-\frac{243}{34850} \sigma_{7}\left(\frac{n}{6}\right) \\
& +\frac{15552}{17425} \sigma_{7}\left(\frac{n}{12}\right)+\frac{44416}{3825} a_{1}(n)+\frac{118784}{3825} a_{1}\left(\frac{n}{2}\right)+\frac{1275264}{425} a_{1}\left(\frac{n}{3}\right)+\frac{11943936}{425} a_{1}\left(\frac{n}{6}\right) \\
& +\frac{35808}{1025} \Delta_{3,8}(n)-\frac{1920}{41} \Delta_{3,8}\left(\frac{n}{2}\right)+\frac{5640192}{1025} \Delta_{3,8}\left(\frac{n}{4}\right)-\frac{1024}{45} a_{4}(n)+\frac{16384}{45} a_{4}\left(\frac{n}{2}\right) \text {, }
\end{aligned}
$$




$$
\begin{aligned}
& N\left(1^{12}, 2^{2}, 6^{2} ; n\right)=\frac{547}{1672800} \sigma_{7}(n)-\frac{547}{1672800} \sigma_{7}\left(\frac{n}{2}\right)-\frac{729}{557600} \sigma_{7}\left(\frac{n}{3}\right) \\
& +\frac{547}{418200} \sigma_{7}\left(\frac{n}{4}\right)+\frac{729}{557600} \sigma_{7}\left(\frac{n}{6}\right)-\frac{17504}{52275} \sigma_{7}\left(\frac{n}{8}\right)-\frac{729}{139400} \sigma_{7}\left(\frac{n}{12}\right) \\
& +\frac{23328}{17425} \sigma_{7}\left(\frac{n}{24}\right)-\frac{4337914}{72675} a_{1}(n)-\frac{1344688}{2565} a_{1}\left(\frac{n}{2}\right)+\frac{2296944}{8075} a_{1}\left(\frac{n}{3}\right) \\
& +\frac{625923392}{218025} a_{1}\left(\frac{n}{4}\right)+\frac{99576}{95} a_{1}\left(\frac{n}{6}\right)+\frac{1940072256}{8075} a_{1}\left(\frac{n}{12}\right) \\
& -\frac{204721}{58425} \Delta_{3,8}(n)-\frac{50435486}{58425} \Delta_{3,8}\left(\frac{n}{2}\right)+\frac{113806456}{58425} \Delta_{3,8}\left(\frac{n}{4}\right) \\
& -\frac{2734079104}{58425} \Delta_{3,8}\left(\frac{n}{8}\right)+\frac{53896}{855} a_{4}(n)+\frac{264992}{513} a_{4}\left(\frac{n}{2}\right)+\frac{552448}{135} a_{4}\left(\frac{n}{4}\right) \\
& -40 a_{2}(n)-\frac{46008}{19} a_{2}\left(\frac{n}{3}\right)+64 a_{3}(n)+\frac{46008}{19} a_{3}\left(\frac{n}{3}\right)+\frac{212544}{19} a_{5}(n) \\
& -\frac{892800}{19} a_{5}\left(\frac{n}{2}\right)-304 a_{6}(n)-\frac{19776}{19} a_{6}\left(\frac{n}{2}\right) \\
& -\frac{87168}{19} a_{7}(n)+\frac{100608}{19} a_{8}(n)+\frac{309504}{19} a_{9}(n) \text {, } \\
& N\left(1^{12}, 2^{2}, 3^{2} ; n\right)=\frac{547}{836400} \sigma_{7}(n)-\frac{547}{836400} \sigma_{7}\left(\frac{n}{2}\right)-\frac{729}{278800} \sigma_{7}\left(\frac{n}{3}\right) \\
& +\frac{547}{418200} \sigma_{7}\left(\frac{n}{4}\right)+\frac{729}{278800} \sigma_{7}\left(\frac{n}{6}\right)-\frac{17504}{52275} \sigma_{7}\left(\frac{n}{8}\right)-\frac{729}{139400} \sigma_{7}\left(\frac{n}{12}\right) \\
& +\frac{23328}{17425} \sigma_{7}\left(\frac{n}{24}\right)-\frac{12235798}{72675} a_{1}(n)-\frac{495656}{855} a_{1}\left(\frac{n}{2}\right)+\frac{28200258}{8075} a_{1}\left(\frac{n}{3}\right) \\
& +\frac{87292864}{72675} a_{1}\left(\frac{n}{4}\right)+\frac{5915376}{95} a_{1}\left(\frac{n}{6}\right)+\frac{1587560256}{8075} a_{1}\left(\frac{n}{12}\right) \\
& -\frac{2518072}{58425} \Delta_{3,8}(n)-\frac{58601612}{58425} \Delta_{3,8}\left(\frac{n}{2}\right)+\frac{5756652856}{58425} \Delta_{3,8}\left(\frac{n}{4}\right) \\
& -\frac{307928704}{58425} \Delta_{3,8}\left(\frac{n}{8}\right)+\frac{183952}{855} a_{4}(n)-\frac{236192}{171} a_{4}\left(\frac{n}{2}\right) \\
& -\frac{2481664}{45} a_{4}\left(\frac{n}{4}\right)-52 a_{2}(n)+\frac{96876}{19} a_{2}\left(\frac{n}{3}\right)+72 a_{3}(n) \\
& -\frac{263088}{19} a_{3}\left(\frac{n}{3}\right)+\frac{1371168}{19} a_{5}(n)+\frac{11576448}{19} a_{5}\left(\frac{n}{2}\right)-448 a_{6}(n) \\
& +\frac{454336}{19} a_{6}\left(\frac{n}{2}\right)-\frac{536448}{19} a_{7}(n)+\frac{584448}{19} a_{8}(n)+\frac{655104}{19} a_{9}(n), \\
& N\left(1^{12}, 2^{4} ; n\right)=\frac{1}{1020} \sigma_{7}(n)-\frac{1}{1020} \sigma_{7}\left(\frac{n}{2}\right)-\frac{1}{255} \sigma_{7}\left(\frac{n}{4}\right)+\frac{256}{255} \sigma_{7}\left(\frac{n}{8}\right) \\
& -\frac{8}{17} a_{1}(n)-16 a_{1}\left(\frac{n}{2}\right)-\frac{512}{17} a_{1}\left(\frac{n}{4}\right)+8 a_{2}(n)+16 a_{3}(n),
\end{aligned}
$$




$$
\begin{aligned}
& N\left(1^{13}, 2,3,6 ; n\right)=\frac{1093}{1672800} \sigma_{7}(n)-\frac{1093}{1672800} \sigma_{7}\left(\frac{n}{2}\right)+\frac{729}{557600} \sigma_{7}\left(\frac{n}{3}\right)-\frac{1093}{836400} \sigma_{7}\left(\frac{n}{4}\right) \\
& -\frac{729}{557600} \sigma_{7}\left(\frac{n}{6}\right)+\frac{17488}{52275} \sigma_{7}\left(\frac{n}{8}\right)-\frac{729}{278800} \sigma_{7}\left(\frac{n}{12}\right)+\frac{11664}{17425} \sigma_{7}\left(\frac{n}{24}\right)+\frac{5030027}{218025} a_{1}(n) \\
& -\frac{662156}{2565} a_{1}\left(\frac{n}{2}\right)-\frac{11089089}{8075} a_{1}\left(\frac{n}{3}\right)+\frac{491700896}{218025} a_{1}\left(\frac{n}{4}\right)-\frac{2587248}{95} a_{1}\left(\frac{n}{6}\right)+\frac{1146292128}{8075} a_{1}\left(\frac{n}{12}\right) \\
& +\frac{990076}{58425} \Delta_{3,8}(n)-\frac{2021554}{58425} \Delta_{3,8}\left(\frac{n}{2}\right)-\frac{2640378532}{58425} \Delta_{3,8}\left(\frac{n}{4}\right)-\frac{2580114752}{58425} \Delta_{3,8}\left(\frac{n}{8}\right) \\
& -\frac{103448}{2565} a_{4}(n)+\frac{453904}{513} a_{4}\left(\frac{n}{2}\right)+\frac{4274944}{135} a_{4}\left(\frac{n}{4}\right)-10 a_{2}(n)-\frac{94446}{19} a_{2}\left(\frac{n}{3}\right)+36 a_{3}(n) \\
& +\frac{177552}{19} a_{3}\left(\frac{n}{3}\right)-\frac{358128}{19} a_{5}(n)-\frac{6681024}{19} a_{5}\left(\frac{n}{2}\right)-\frac{246944}{19} a_{6}\left(\frac{n}{2}\right)+\frac{181056}{19} a_{7}(n) \\
& -\frac{191616}{19} a_{8}(n)-\frac{18048}{19} a_{9}(n) \text {, } \\
& N\left(1^{14}, 6^{2} ; n\right)=\frac{547}{836400} \sigma_{7}(n)-\frac{547}{836400} \sigma_{7}\left(\frac{n}{2}\right)-\frac{729}{278800} \sigma_{7}\left(\frac{n}{3}\right)+\frac{547}{418200} \sigma_{7}\left(\frac{n}{4}\right) \\
& +\frac{729}{278800} \sigma_{7}\left(\frac{n}{6}\right)-\frac{17504}{52275} \sigma_{7}\left(\frac{n}{8}\right)-\frac{729}{139400} \sigma_{7}\left(\frac{n}{12}\right)+\frac{23328}{17425} \sigma_{7}\left(\frac{n}{24}\right)-\frac{266098}{72675} a_{1}(n) \\
& -\frac{1689416}{2565} a_{1}\left(\frac{n}{2}\right)-\frac{60616242}{8075} a_{1}\left(\frac{n}{3}\right)+\frac{989968192}{218025} a_{1}\left(\frac{n}{4}\right)-\frac{8818848}{95} a_{1}\left(\frac{n}{6}\right)+\frac{2292584256}{8075} a_{1}\left(\frac{n}{12}\right) \\
& -\frac{894472}{58425} \Delta_{3,8}(n)-\frac{77504252}{58425} \Delta_{3,8}\left(\frac{n}{2}\right)-\frac{5555962184}{58425} \Delta_{3,8}\left(\frac{n}{4}\right)-\frac{5160229504}{58425} \Delta_{3,8}\left(\frac{n}{8}\right) \\
& +\frac{15952}{855} a_{4}(n)+\frac{1063456}{513} a_{4}\left(\frac{n}{2}\right)+\frac{8549888}{135} a_{4}\left(\frac{n}{4}\right)-28 a_{2}(n)-\frac{188892}{19} a_{2}\left(\frac{n}{3}\right)+56 a_{3}(n) \\
& +\frac{355104}{19} a_{3}\left(\frac{n}{3}\right)-\frac{650592}{19} a_{5}(n)-\frac{13362048}{19} a_{5}\left(\frac{n}{2}\right)-448 a_{6}(n)-\frac{493888}{19} a_{6}\left(\frac{n}{2}\right) \\
& +\frac{362112}{19} a_{7}(n)-\frac{383232}{19} a_{8}(n)-\frac{36096}{19} a_{9}(n) \text {, } \\
& N\left(1^{14}, 3^{2} ; n\right)=\frac{547}{418200} \sigma_{7}(n)-\frac{729}{139400} \sigma_{7}\left(\frac{n}{3}\right)-\frac{17504}{52275} \sigma_{7}\left(\frac{n}{4}\right)+\frac{23328}{17425} \sigma_{7}\left(\frac{n}{12}\right) \\
& -\frac{1045712}{11475} a_{1}(n)-\frac{4395392}{11475} a_{1}\left(\frac{n}{2}\right)-\frac{2133216}{425} a_{1}\left(\frac{n}{3}\right)-\frac{14691456}{425} a_{1}\left(\frac{n}{6}\right)-\frac{47152}{1025} \Delta_{3,8}(n) \\
& -\frac{64624}{25} \Delta_{3,8}\left(\frac{n}{2}\right)-\frac{1196032}{1025} \Delta_{3,8}\left(\frac{n}{4}\right)+\frac{22208}{135} a_{4}(n)+\frac{155648}{135} a_{4}\left(\frac{n}{2}\right)+6912 a_{5}(n) \\
& -896 a_{6}(n) \text {, } \\
& \begin{array}{l}
N\left(1^{14}, 2^{2} ; n\right)=\frac{1}{510} \sigma_{7}(n)-\frac{1}{510} \sigma_{7}\left(\frac{n}{2}\right)-\frac{1}{255} \sigma_{7}\left(\frac{n}{4}\right)+\frac{256}{255} \sigma_{7}\left(\frac{n}{8}\right)+\frac{52}{17} a_{1}(n) \\
+48 a_{1}\left(\frac{n}{2}\right)-\frac{512}{17} a_{1}\left(\frac{n}{4}\right)+8 a_{2}(n)+16 a_{3}(n), \\
N\left(1^{16} ; n\right)=\frac{1}{255} \sigma_{7}(n)-\frac{2}{255} \sigma_{7}\left(\frac{n}{2}\right)+\frac{256}{255} \sigma_{7}\left(\frac{n}{4}\right)+\frac{512}{17} a_{1}(n)+\frac{8192}{17} a_{1}\left(\frac{n}{2}\right),
\end{array}
\end{aligned}
$$




\section{References}

[1] Ono, K. (2004) The Web of Modularity: Arithmetic of the Coefficients of Modular Forms and Q-Series. CBMS Regional Conf. Series in Math. 102, Amer. Math. Soc., Providence.

[2] Alaca, A., Alaca, S. and Williams, K.S. (2010) Fourteen Octonary Quadratic Forms. International Journal of Number Theory, 6, 37-50. http://dx.doi.org/10.1142/S179304211000279X

[3] Alaca, S. and Williams, K.S. (2010) The Number of Representations of a Positive İnteger by Certain Octonary Quadratic Forms. Functiones et Approximatio Commentarii Mathematici, 43, 45-54. http://dx.doi.org/10.7169/facm/1285679145

[4] Alaca, S. and Kesicioglu, Y. (2014) Representatıons by Certain Octonary Quadratıc Forms Whose Coefficients Are 1, 2, 3 and 6. International Journal of Number Theory, 10, 133-150. http://dx.doi.org/10.1142/S1793042113500851

[5] Alaca, S. and Kesicioglu, Y. (2015) Representatıons by Certaın Octonary Quadratıc Forms with Coefficients 1, 2, 3, and 6. Integers, 15, 1-9.

[6] Jacobi, C.G.J. (1969) Fundamenta Nova Theoriae Functionum Ellipticarum. In: Gesammelte Werke, Erster Band Chelsea Publishing Co., New York, 49-239, 285-290.

[7] Kendirli, B. (2012) Cusp Forms in $S_{4}\left(\Gamma_{0}(47)\right)$ and the Number of Representations of Positive İntegers by Some Direct Sum of Binary Quadratic Forms with Discriminant -47. International Journal of Mathematics and Mathematical Sciences, 2012, Article ID: 303492, 10 p.

[8] Kendirli, B. (2015) Evaluation of Some Convolution Sums by Quasimodular Forms. European Journal of Pure and Applied Mathematics, 8, 81-110.

[9] Kendirli, B. (2014) Evaluation of Some Convolution Sums andthe Representation Numbers. Ars Combinatoria, CXVI, 65-91.

[10] Kendirli, B. (2015) Evaluation of Some Convolution Sums and Representation Numbers of Quadratic Forms of Discriminant 135. British Journal of Mathematics and Computer Science, 6, 494-531. http://dx.doi.org/10.9734/BJMCS/2015/13973

[11] Kokluce, B. (2013) The Representation Numbers of Three Octonary Quadratic Forms. International Journal of Number Theory, 9, 505-516. http://dx.doi.org/10.1142/S1793042112501461

[12] Ramakrishnan B. and Sahu, B. (2013) Evaluation of the Convolution Sums $\sum_{l+15 m=n} \sigma(l) \sigma(m)$ and $\sum_{3 l+5 m=n} \sigma(l) \sigma(m)$ and an Application. International Journal of Number Theory, 9, 799-809. http://dx.doi.org/10.1142/S179304211250162X

[13] Alaca, A., Alaca, S. and Williams, K.S. (2010) Sextenary Quadratıc Forms and an Identity of Kleın and Frıcke. International Journal of Number Theory, 6, 169-183. http://dx.doi.org/10.1142/S1793042110002880

[14] Alaca, A., Alaca, S. and Williams, K.S. (2008) Liouvılle's Sextenary Quadratıc Forms $x^{2}+y^{2}+z^{2}+t^{2}+2 u^{2}+2 v^{2}$, $x^{2}+y^{2}+2 z^{2}+2 t^{2}+2 u^{2}+2 v^{2}$ and $x^{2}+2 y^{2}+2 z^{2}+2 t^{2}+2 u^{2}+4 v^{2}$. Far East Journal of Mathematical Sciences, 30, 547-556.

[15] Alaca, A., Alaca, S. and Williams, K.S. (2009) Some New Theta Function Identities with Applications to Sextenary Quadratic Forms. Journal of Combinatorics and Number Theory, 1, 89-98.

[16] Berkovich, A. and Yeşilyurt, H. (2009) On the Representations of İntegers by the Sextenary Quadratic Form $x^{2}+y^{2}+z^{2}+7 s^{2}+7 t^{2}+7 u^{2}$ and 7-Cores. Journal of Number Theory, 129, 1366-1378. http://dx.doi.org/10.1016/j.jnt.2008.09.001

[17] Xia, E.X.W., Yao, O.X.M. and Zhao, A.F.Y. (2015) Representation Numbers of Five Sextenary Quadratic Forms. Colloquium Mathematıcum, 138, 247-254. http://dx.doi.org/10.4064/cm138-2-9

[18] Kendirli, B. (2012) Cusp Forms in $S_{4}\left(\Gamma_{0}(79)\right)$ and the Number of Representations of Positive İntegers by Some Direct Sum of Binary Quadratic forms With Discriminant -79. Bulletin of the Korean Mathematical Society, 49, 529-572. http://dx.doi.org/10.4134/BKMS.2012.49.3.529

[19] Kendirli, B. (2015) The Number of Representations of Positive Integers by Some Direct Sum Of Binary Quadratic Forms With Discriminant -103. IJEMME International Journal of Electronics, Mechanical, and Mecathronics Engineering, 5, 979-1007.

[20] Köhler, G. (2011) Eta Products and Theta Series Identities. Springer-Verlag, Berlin.

[21] Diamond, F. and Shurman, J. (2005) A First Course in Modular Forms (Graduate Texts in Mathematics, Vol. 228). Springer, Berlin. 